\title{
Exposure-Relevant Ozone Chemistry in Occupied Spaces
}

\author{
Beverly Kaye Coleman \\ Ph.D. Thesis
Department of Civil and Environmental Engineering University of California, Berkeley
and
Environmental Energy Technologies Division
University of California
Berkeley, California 94720 \\ Ernest Orlando Lawrence Berkeley National Laboratory
}

Spring 2009

This work was supported by the National Science Foundation Graduate Fellowship, the California Air Resources Board (Contract No. 01-336), and the U.S. Federal Aviation Administration (FAA) Office of Aerospace Medicine through the Air Transportation Center of Excellence for Airliner Cabin Environment Research (ACER). All work at LBNL was conducted under US Department of Energy Contract No. DE-AC02$05 \mathrm{CH} 11231$. 
Exposure-Relevant Ozone Chemistry in Occupied Spaces

(C) 2009

by

Beverly Kaye Coleman 


\begin{abstract}
Exposure-Relevant Ozone Chemistry in Occupied Spaces

by

Beverly Kaye Coleman

Doctor of Philosophy in Engineering-Civil and Environmental Engineering

University of California, Berkeley

Professor William W Nazaroff, Chair
\end{abstract}

Ozone reacts with alkenes and other organic compounds that contain carboncarbon double bonds to form oxidation byproducts such as aldehydes, ketones, carboxylic acids, radicals, and secondary organic aerosol. Ozone-reactive species such as terpenes and unsaturated fatty acids are ubiquitous in the air and on surfaces in indoor environments. The most prevalent source of ozone indoors is ventilation of the space with ozone-containing ambient air, although some appliances that are used indoors also produce ozone. Reactions with surfaces are the ever-present and dominant sink of ozone and source of oxidation byproducts. Gas-phase ozone reactions must compete with the ventilation rate; they can be intermittently important ozone consumers and byproduct generators. Ozone-alkene reactions are chain-initiating because radicals are formed that 
continue to react. The hydroxyl radical $(\mathrm{OH})$ is a major secondary byproduct of ozonealkene chemistry, and it is a less-selective, faster reacting oxidizer than ozone. Thus, species that do not readily react with ozone, i.e. compounds that do not contain a carboncarbon double bond, may also be oxidized by the $\mathrm{OH}$ radical in ozone-initiated chemistry.

In this dissertation, the chemical and physical factors that affect transformation of ozone into other airborne pollutants in occupied indoor environments are explored. Ozone-initiated reaction with, and byproduct formation from, reactive gas-phase and surface-phase species common to indoor settings were investigated in four studies. Byproduct types and formation rates were characterized in laboratory experiments. Byproduct concentrations and exposures were predicted in various indoor environments using experimental data and a model that predicts ozone transport and uptake and byproduct formation and fate.

When both ozone and terpenes are present in indoor settings, terpenes can be a strong sink of ozone and source of gas- and particle-phase byproducts. I investigated secondary organic aerosol formation from the reaction of ozone with terpene-containing consumer products under conditions relevant for residential and commercial buildings. Gas-phase consumer product emissions and then ozone were introduced into a continuously ventilated 198-L chamber. At the onset of ozone addition, a nucleation event occurred, and nucleation and growth continued to occur as long as the reagents were introduced into the chamber. The particle formation and growth behavior in these experiments mimicked SOA dynamics from ozone-terpene reactions measured in actual buildings. The full particle size distribution was continuously monitored using an optical 
particle counter and scanning mobility particle sizer. The resulting ultrafine and fine particle concentrations were in the range of 10 to $>300 \mu \mathrm{g} \mathrm{m}^{-3}$. Particle nucleation and growth dynamics under indoor conditions were characterized using the methods commonly applied to atmospheric nucleation events.

Commercial passenger aircraft can encounter elevated stratospheric ozone levels at cruising altitude, and because aircraft cabins are continuously ventilated, significant ozone levels can be present in the aircraft cabin. Reactions with fixed cabin surfaces and surfaces associated with passengers consume ozone and generate byproducts. I conducted chamber experiments at flight-relevant conditions to determine ozone uptake and byproduct emissions from individual materials found in the aircraft cabin environment. The materials tested included new and used samples of carpet, seat fabric, and plastics, and laundered and worn clothing fabric. For all materials, emission rates were higher with ozone than without. Ozone deposition velocities and reaction probabilities, and byproduct emission rates and byproduct yields, were determined for each of the surface categories. The most commonly detected byproducts included $\mathrm{C}_{1}-\mathrm{C}_{10}$ saturated aldehydes and skin oil oxidation products. A model of mass transport and uptake was employed to extrapolate results from chamber experiments to the cabin environment. I estimated the distribution of total byproduct levels using a Monte Carlo simulation of the cabin environment with three model parameters: byproduct yield, ozone level, and retention ratio. Airborne oxidation byproduct levels are predicted to be similar to ozone levels in the cabin, which have been found to be tens to low hundreds of ppb in the absence of an ozone converter. I also used this model to predict the concentrations of certain byproducts in the cabin, and exposure to these byproducts were compared in three important 
environments - an aircraft cabin, a residential building, and outdoors - using inhalation intake rate as a metric. For the byproducts examined, intake in the aircraft cabin can be similar to intake in buildings for those who spend a significant amount of time flying, such as crew members, despite much more time being spent in buildings, owing to higher levels of byproducts in the cabin.

Surface materials may be inherently reactive with ozone or may have ozonereactive residues applied during manufacture or use of the surface. I developed a model of ozone uptake by, and byproduct emission from, residual chemicals on surfaces. The model predicts the time-dependent rate of ozone consumption, residue consumption, and byproduct formation with the following inputs: residue surface concentration, ozone concentration, reactivity of the residue and the surface, near-surface airflow conditions, and byproduct yield. The effects of model input parameters on ozone uptake and byproduct formation were explored. There is potential for this model to help elucidate the dynamic ozone uptake behavior such as "aging" and "regeneration" - the gradual reduction in reactivity of material over the course of ozone exposure, and the rebound in material reactivity exhibited after a material has been exposed to ozone-free air for a period of time in between ozone exposures, respectively.

Permethrin is a residual (surface-bound) insecticide commonly used in aircraft cabins. The possibility that ozone could react with permethrin to form phosgene was investigated. From the literature, it was determined that surface levels of permethrin and airborne levels of ozone were sufficient to potentially form phosgene at a level of concern based on established health standards for phosgene. A derivatization technique was developed to detect phosgene at low levels, and experiments were conducted in which 
permethrin-coated glass plates and aircraft cabin materials were exposed to ozone under flight-relevant conditions. Phosgene was not detected in these experiments, and on the basis of the research conducted, it does not appear likely that ozone-initiated oxidation or OH-related oxidation of permethrin is a major route of degradation for permethrin in indoor spaces. Permethrin likely has a very low reactivity with ozone owing to the presence of chlorine atoms adjacent to the double bond in permethrin. A mathematical model of ozone transport and uptake was employed to estimate an upper bound on phosgene formation and levels in an aircraft cabin. The reaction probability of permethrin is estimated to be $<10^{-7}$ and the cabin concentration of phosgene to be $<1 \mu \mathrm{g} \mathrm{m}^{-3}$. It was determined that phosgene formation, if it occurs in the aircraft cabin, is not likely to exceed the relevant, health-based phosgene exposure guidelines.

The research presented here provides evidence that ozone, a ubiquitous ambient pollutant, is transformed into other airborne pollutants in the indoor environment where we spend the majority of our time. Ozone-initiated chemistry lowers the indoor ozone level but may generate oxidation byproducts that can be as harmful, or more so, than ozone itself. The type and amount of byproducts that result from ozone reactions with common indoor surfaces, surface residues, and vapors were determined, pollutant concentrations were related to occupant exposure, and frameworks were developed to predict byproduct concentrations under various indoor conditions. This work also helped to elucidate the role of occupants in indoor ozone chemistry. Human skin oil is highly reactive with ozone, and oxidation byproducts are potentially formed very near the breathing zone. Ozone-initiated reactions that occur on or very near occupants, and the 
control of ozone to reduce exposure to oxidation byproducts in occupied spaces, are emerging issues in indoor ozone chemistry. 


\section{ACKNOWLEDGMENTS}

I thank my family and loved ones whose love and support made this accomplishment possible. To my parents, Mike and Kaye, thank you for always believing in me. I am grateful to them for paving the way for me to accomplish my dreams. Thanks to my sisters, Deborah and Michelle, who have made me laugh and gave me perspective on life. To Matt, my boyfriend and best friend, your enthusiastic spirit kept me buoyant, and your encouragement has kept me going. I am so happy to finally share this achievement with you. My thanks to you and your family - Nursen, Fertaç, and Daphne for helping me enjoy the journey and celebrate the milestones.

I have been fortunate to have brilliant mentors, and I owe my academic success to them. Above all, I want to thank my research advisor, Bill Nazaroff, whose dedication, rigor, and scientific ability are unparalleled. He inspired me through his fascination with knowledge, his standard of excellence, and his commitment to teaching inside and outside the classroom. Because of Bill's investment in my research and professional development, I have become a better communicator and a more thorough scientist. I would also like to thank my committee members Rob Harley and Allen Goldstein for their insights that greatly improved my dissertation.

I gained invaluable experience while working at Lawrence Berkeley National Laboratory. Al Hodgson, Brett Singer, and Randy Maddalena provided patient oversight and great feedback. Hugo Destaillats has been an integral part of every one of my research projects. I am indebted to him for being not only a wonderful mentor but also a 
great friend. To Melissa Lunden, you have been there for me in so many capacities throughout the years, and I am thankful to have your friendship and guidance. In regards to my dissertation, you taught me Igor and you deserve much of the credit for Chapter 2 .

I have had many fantastic mentors outside of Berkeley. Rich Corsi at the University of Texas at Austin sparked my interest in research and indoor air quality when I was an undergraduate. His charisma and encouragement have carried me further than even he could imagine. I have never had more fun while working than I did with Ray Wells and Jason Ham at the National Institute of Occupational Safety and Health in West Virginia. I thank Ray for the opportunity to collaborate with his group and for making Chapter 5 of this dissertation possible. Charlie Weschler and Glenn Morrison have been ubiquitous sources of inspiration throughout my academic career. It has been such a pleasure to learn from all of you. Thanks for giving me a glimpse of the view from the shoulders of giants.

A huge thanks to all of the Airheads, past and present, who have made Berkeley such a wonderful experience. Words cannot express my gratitude to Sharon, Seema, and Arman for being such great friends and listeners. Thanks to George, Dev, Juli, and Nasim, who are dear friends and made Davis Hall a fun place to be. The generation before me - Julian, Abby, Garvin, Rengie, Shannon, and Priya - gave me lots of good advice and set a high standard. The young blood of the group - Josh, Drew, and Tim are so impressive, and I know they will make the group proud.

I was lucky to meet some of my best friends - Rohit, Jonathan, and Jack - on my first day at graduate school at Cal. I have been fortunate to be a part of a generation where I have always had great female friends in the sciences. To Amara, Rachelle, Katie, 
Christine, Stephannie, and Kellye, your friendship has meant so much to me and made this process fun. 


\section{TABLE OF CONTENTS}

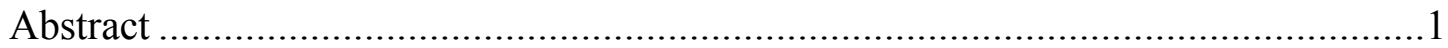

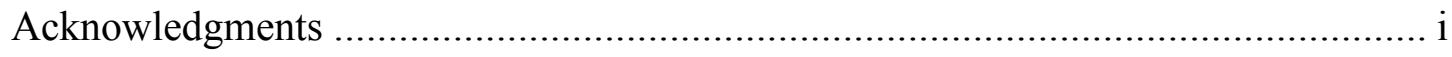

Table of contents.......................................................................................... iv

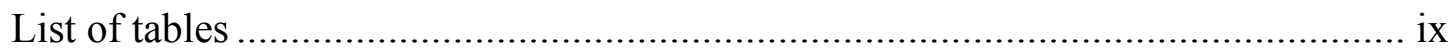

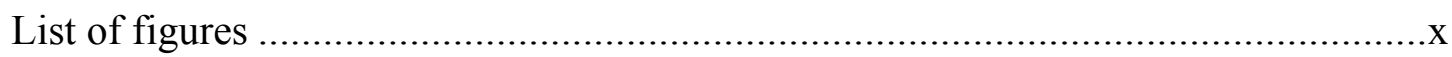

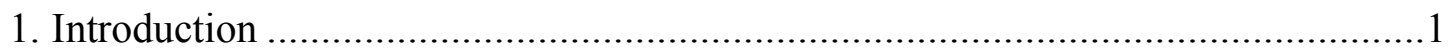

1.1. Background: Indoor air quality and ozone chemistry ............................

1.2. Introduction to dissertation projects ..................................................11

2. Secondary organic aerosol from ozone reactions with cleaning products ..............15

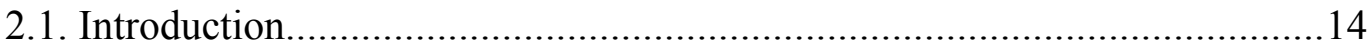

2.2. Experimental conditions ......................................................... 17

2.3. Particle measurement and analysis................................................... 19

2.3.1. Instruments.................................................................... 19

2.3.2. Alignment routine.......................................................... 21

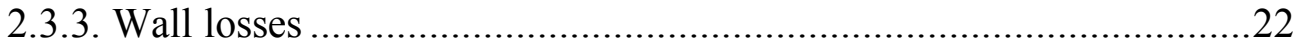

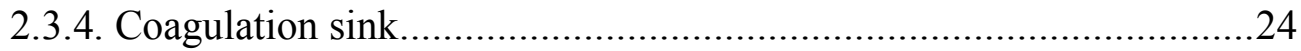

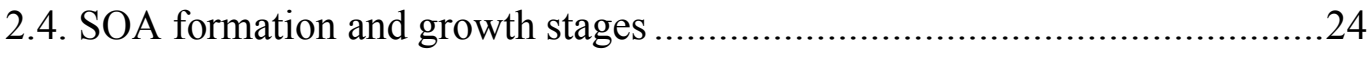

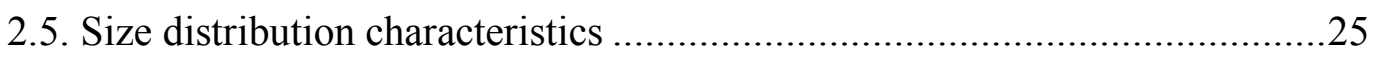

2.5.1. Effect of ozone level and air-exchange rate on mass and yield.........27

2.5.2. Effect of RH, order of reagent addition, and ambient seed particles ..29

2.6. Particle formation and growth characteristics .................................. 30

2.6.1. Cycle of particle formation and growth..................................... 30 
2.6.2. Nucleation subsequent to the initial event.

2.6.3. Modeling total particle mass formation ....................................... 35

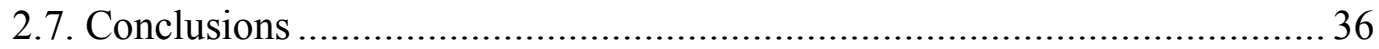

2.A. Appendix: Codes for particle distribution analysis .............................. 49

2.B. Appendix: Additional experimental data ........................................ 58

3. Ozone-surface reactions, byproduct formation, and byproduct exposure in the

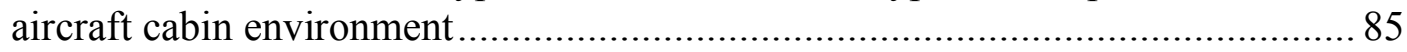

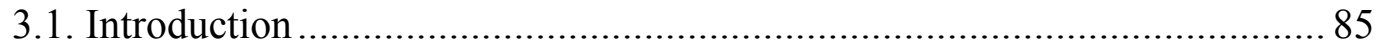

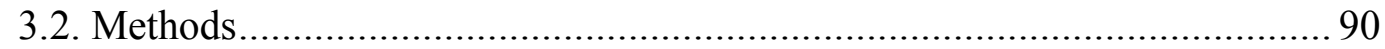

3.2.1. Evaluating ozone deposition and byproduct emissions .................. 90

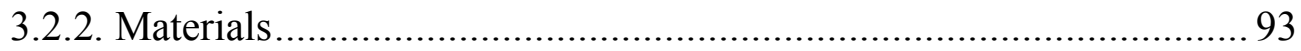

3.2.3. Experimental apparatus .................................................. 95

3.2.4. Chemical sampling and analysis............................................... 95

3.3. Ozone deposition experiments .................................................. 97

3.3.1. Experimental protocol....................................................... 97

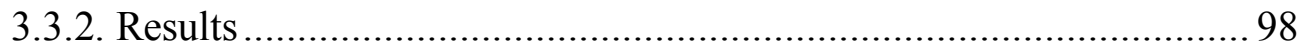

3.3.3. Aging and regeneration in screening experiments...................... 100

3.4. Byproduct emissions experiments ................................................. 100

3.4.1. Experimental protocol...................................................... 100

3.4.2. Emissions from cabin materials............................................ 101

3.4.3. Emissions from clean and worn fabric: skin oil chemistry ........... 103

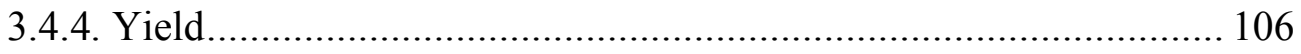

3.4.5. Effect of environmental factors ........................................... 106

3.4.6. Aging and regeneration in emissions experiments ..................... 108

3.5. Contributions of surface ozone reactivity to cabin air quality ................. 109 


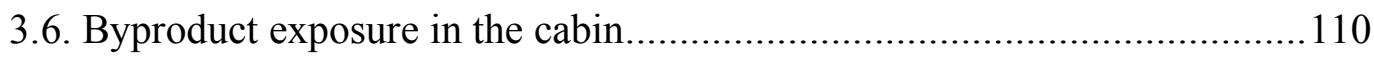

3.6.1. Predicting byproduct levels............................................................... 111

3.6.2. Estimating byproduct intake …………………...............................115

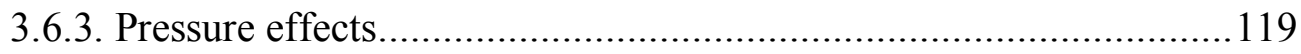

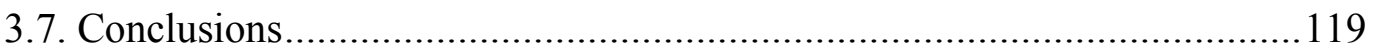

3.A. Appendix: Extrapolating chamber data to real environments.....................139

3.B. Appendix: Surface-to-volume ratios for various aircraft...........................142

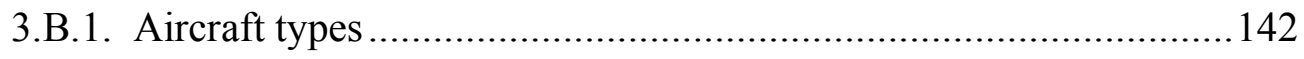

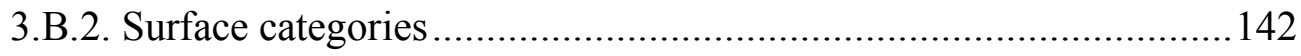

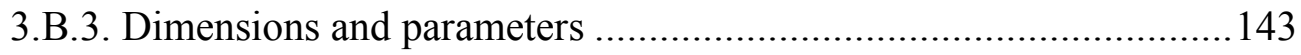

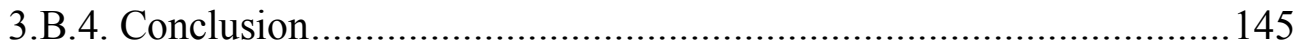

3.C. Appendix: Additional experimental data ...................................................149

4. Ozone reactions with, and byproduct formation from, residual chemicals on

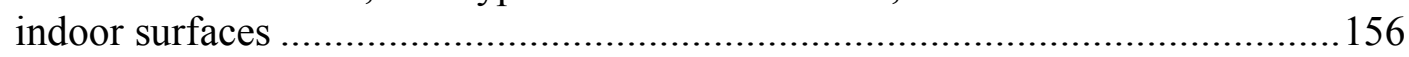

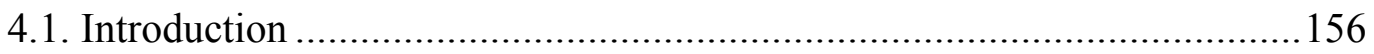

4.2. Ozone uptake by residual compound .....................................................158

4.2.1. Conceptual illustration and model scope ………............................158

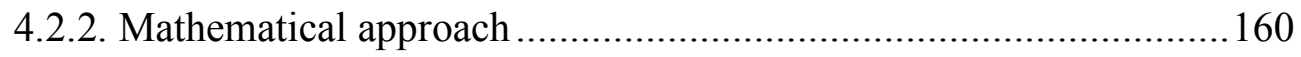

4.3. Time-dependent model of deposition and byproduct formation:

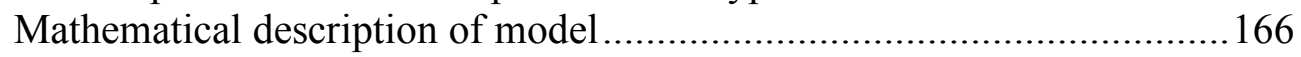

4.4. Parametric investigation of model constant ozone level............................173

4.4.1. Base material and residual characteristics: Model inputs .................173

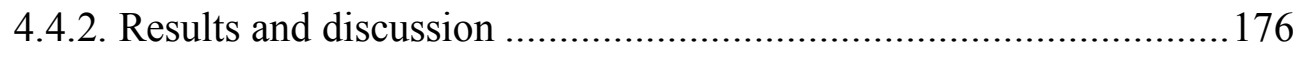

4.5. Application of model to chamber experiments with variable ozone level.. 179

4.6. Implications for indoor environments and conclusions .............................180 
5. Investigation of ozone-induced decomposition of surface-bound permethrin...... 195

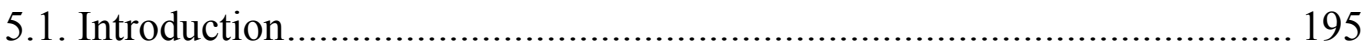

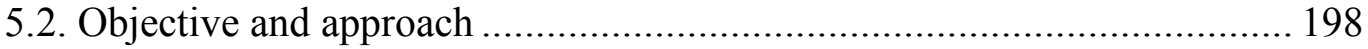

5.3. Characterization of potential for phosgene formation........................... 198

5.3.1. Pyrethroid surface concentrations .......................................... 199

5.3.2. Potential phosgene formation ................................................ 201

5.3.3. Reactivity of permethrin ............................................... 203

5.3.4. Background information for phosgene ................................. 205

5.3.5. Phosgene exposure guidelines ........................................... 206

5.3.6. Phosgene chemical characteristics ........................................ 207

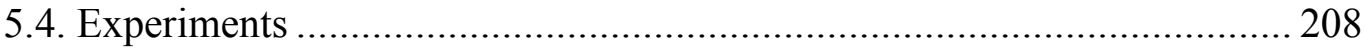

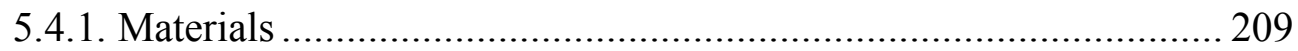

5.4.2. Detection method and chemical analysis................................ 210

5.4.3. Calibrations ...................................................................... 211

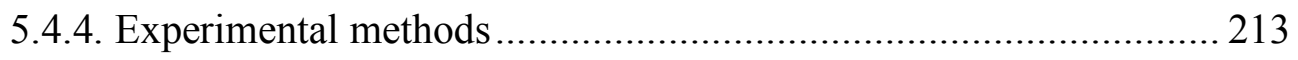

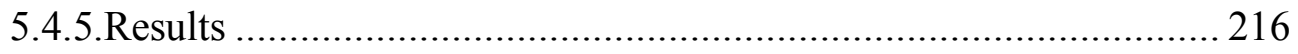

5.5. Implications for phosgene exposure in the cabin: establishing an upper-bound on phosgene levels in aircraft cabins............................. 217

5.6. Permethrin, ozone, and phosgene in indoor environments.................... 221

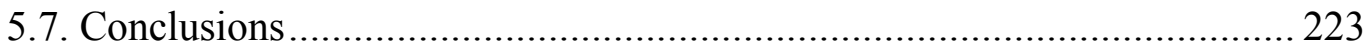

5.A. Preliminary experiments to detect phosgene from ozone reaction with

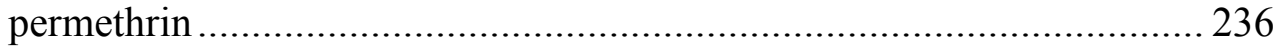

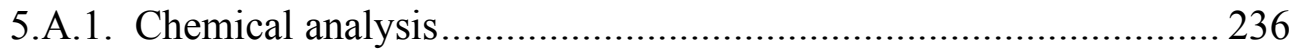

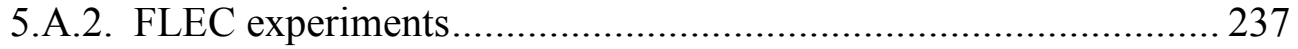

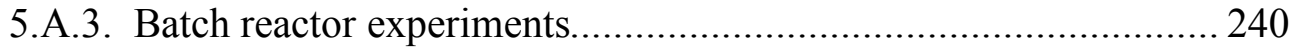


5.A.4. CMFR reactor experiments

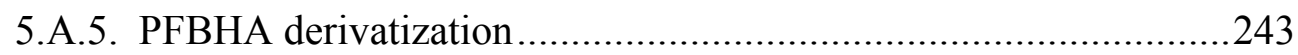

6. Summary and recommendations of future research............................................249

6.1. Secondary organic aerosol from indoor ozone-terpene reactions................250

6.2. Ozone consumption and byproduct formation of cabin surfaces ...............252

6.3. Modeling ozone reactions with residue covered surfaces...........................254

6.4. Ozone-induced oxidation of surface-bound permethrin ............................256

6.5. Indoor ozone chemistry trends and solutions ............................................258

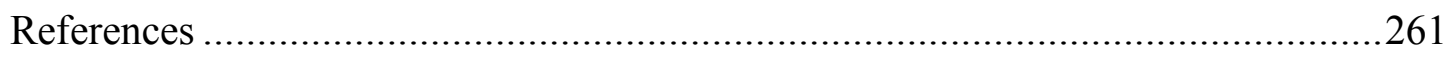




\section{LIST OF TABLES}

Table 2-1: Conditions for experiments where ozone and terpene-containing consumer product vapors were mixed in a continuously ventilated $198 \mathrm{~L}$ Teflon-lined chamber.

Table 2-2: Modeled three-mode lognormal SOA distribution parameters

Table 2-3: Measured and modeled SOA number and mass concentrations 40

Table 2-4: SOA number and mass formation rates 41

Table 2B-1: Supply and residual terpene levels for OOD experiments. 57

Table 2B-2: Supply and terpene residual levels for AFR experiments................... 58

Table 2B-3: Supply and terpene residual levels for POC experiments.

Table 3-1: Initial, final and 3-h average ozone deposition velocities and 3-h average reaction probability for 22 cabin materials

Table 3-2: Ozone regeneration experiments with used seat fabric

Table 3-3: Flight attendant's estimated weekly inhalation intake rate of three ozone byproducts in various environments....

Table 3B-1: Cabin dimensions and surface-to-volume calculations for various aircraft

Table 3C-1: Yields of oxidation byproducts from laundered cotton in various conditions during 90-minute exposure to ozone

Table 3C-2: Yields of oxidation byproducts from new and used cabin materials and laundered and soiled clothing fabrics.

Table 3C-3: Surface category-averaged deposition velocity, yield, and surface-to-volume ratio and contributions of each surface to area, ozone consumption, and byproduct emissions

Table 5-1: The effect of chlorine substitution on ozone reaction rate with simple alkenes

Table 5-2: Conditions of permethrin oxidation experiments with derivatization detection technique 


\section{LIST OF FIGURES}

Figure 2-1: Diagram of experimental apparatus for the measurement of byproducts from gas-phase reactions of ozone and terpene-containing consumer products

Figure 2-2: Particle size-distribution evolution as measured with an SMPS for six experiments in which vapor from a pine-oil cleaner (POC) was combined with ozone under different conditions.

Figure 2-3: Characteristic stages of particle formation and growth in chamber experiments, illustrated for experiment AFR-HH.

Figure 2-4: Size distributions measured with an SMPS and an OPC and fit using the sum of three lognormal distributions

Figure 2-5: Effect of ozone level and air-exchange rate on steady-state particle mass concentration and yield.

Figure 2-6: Comparison of measured particle number decay with that expected from removal by ventilation alone, for experiment POC-MH during stage 2

Figure 2-7: Modeled and measured particle mass concentrations vs. time for three experiments using the orange-oil degreaser (OOD), which contained d-limonene.

Figure 2A-1: Example output for the function "CalcCondensationCharTime".

Figure 2B-1: Color-scale for SMPS measurements and SMPS measurements for experiment OOD-HH.

Figure 2B-2: SMPS measurements for experiment OOD-HL. ............................61

Figure 2B-3: SMPS measurements for experiment OOD-MH. .............................62

Figure 2B-4: SMPS and OPC measurements for experiment AFR-HH..................63

Figure 2B-5: SMPS and OPC measurements for experiment AFR-HL. ..................64

Figure 2B-6: SMPS and OPC measurements for experiment AFR-MH .................65

Figure 2B-7: SMPS and OPC measurements for experiment POC-VH..................66

Figure 2B-8: SMPS and OPC measurements for experiment POC-HH1 ................67 
Figure 2B-9: SMPS measurements for experiment POC-HH2 …...................... 68

Figure 2B-10: SMPS measurements for experiment POC-HL .............................. 69

Figure 2B-11: SMPS measurements for experiment POC-MH............................. 70

Figure 2B-12: SMPS and OPC measurements for experiment POC-LH................. 71

Figure 2B-13: SMPS and OPC measurements for experiment POC-NOx .............. 72

Figure 2B-14: SMPS and OPC measurements for experiment POC-Rev................ 73

Figure 2B-15: SMPS and OPC measurements for experiment POC-Seed ............... 74

Figure 2B-16: SMPS measurements for experiment POC-Dry ............................ 75

Figure 2B-17: Measured and modeled particle size distribution data from stage 4 of experiment OOD-HH and OOD-MH ............................................. 76

Figure 2B-18: Measured and modeled particle size distribution data from stage 4 of experiment OOD-HL ................................................................ 77

Figure 2B-19: Measured and modeled particle size distribution data from stage 4 of experiment AFR-HH and AFR-HL .................................................. 78

Figure 2B-20: Measured and modeled particle size distribution data from stage 4 of experiment AFR-MH and POC-VH................................................ 79

Figure 2B-21: Measured and modeled particle size distribution data from stage 4 of experiment POC-HH1 and POC-HH2 .............................................. 80

Figure 2B-22: Measured and modeled particle size distribution data from stage 4

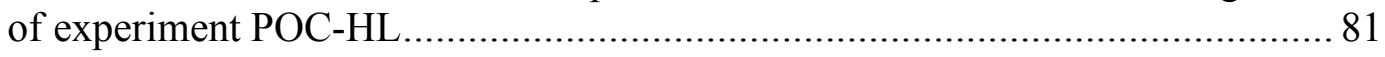

Figure 2B-23: Measured and modeled particle size distribution data from stage 4

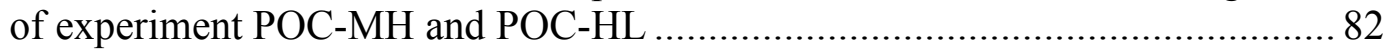

Figure 2B-24: Measured and modeled particle size distribution data from stage 4 of experiment POC-NOx and POC-Rev.

Figure 2B-25: Measured and modeled particle size distribution data from stage 4 of experiment POC-Seed and POC-Dry .

Figure 3-1: Examples of atmospheric ozone profiles taken from Müller et al. (2003) with superimposed ozone layer and typical cruising altitude for commercial aircraft.

Figure 3-2: Schematic diagram of experimental set-up for emissions experiments. 126 
Figure 3-3: Ozone deposition velocity (3-h average) to three common fabrics: cotton, wool, and polyester.

Figure 3-4: Screening experiment data mapped onto deposition velocity model .....128

Figure 3-5: Ozone reactivity profile of four materials exposed to an identical set of conditions.

Figure 3-6: Emission rates of selected volatile organic compounds from (a) new and used common cabin materials and (b) laundered and soiled clothing fabrics

Figure 3-7: Squalene and its detected, volatile oxidation products.

Figure 3-8: Composition of sebum and skin oil. 132

Figure 3-9: The most prevalent fatty acids in human skin oil 133

Figure 3-10: Molar yields of the sum of $\mathrm{C}_{1}$ through $\mathrm{C}_{10}$ saturated aldehydes, acetone, and 6-MHO from new and used cabin materials and laundered and soiled clothing fabrics.

Figure 3-11: Emission rates from cotton exposed to varying levels of ozone under different relative humidity conditions.

Figure 3-12: Contribution of major cabin surfaces to (a) total cabin surface area, (b) ozone consumption, and (c) byproduct emissions.................................136

Figure 3-13: Typical airflow patterns in a wide-body plane.

Figure 3-14: Input (yield, retention ratio, and cabin ozone level) and output distributions for Monte Carlo simulation of peak-hour byproduct levels

Figure 3A-1: Steps to calculate the ozone deposition velocity in a real environment given chamber data and information about flow conditions in the real environment.

Figure 3A-2: Steps to calculate the ozone byproduct concentration using deposition velocities and surface-to-volume ratios of individual materials

Figure 3B-1: Surface-to-volume ratios for four passenger aircraft. 148

Figure 3C-1: Ozone reactivity profile data for all ozone deposition experiments ....152

Figure 4-1: Illustration of residual chemical reactivity model 182

Figure 4-2: Illustration of ozone deposition model 183 
Figure 4-3: The effect of residual reactivity and residual coverage on overall ozone deposition to the surface ...................................................... 184

Figure 4-4: Illustration of flat and textured surfaces ...................................... 185

Figure 4-5: Oleic acid and its oxidation products ............................................. 186

Figure 4-6: Parameter inputs and model results for oleic acid and cleaning product examples

Figure 4-7: Time-dependent (a) overall deposition velocity, $v_{d}$, and (b) overall reaction probability, $\gamma$, to a surface with base reactivity, $\gamma_{B}=10^{-7}$, and varying residue reaction probability

Figure 4-8: Time-dependent ozone deposition velocity with varying base and residue reactivities.

Figure 4-9: Effects on deposition velocity of various model parameters: initial monolayer or fractional surface coverage, surface multiplication factor, friction velocity, and steady indoor ozone level

Figure 4-10: Model fit to ozone data measured in a chamber experiment with new plastic, soiled wool, and new carpet.....

Figure 5-1: Chemical structures of the common cabin insecticides phenothrin and permethrin and potential reaction of permethrin and ozone to form phosgene

Figure 5-2: Surface concentrations of phenothrin and permethrin measured in four cabin studies and one chamber study.

Figure 5-3: Schematic of the derivatization technique for phosgene

Figure 5-4: Derivatization reaction for phosgene with dimercaptotoluene and triethylamine based on the work of Muir et al. (2005)

Figure 5-5: Calibration curves for direct liquid phosgene derivatization 232

Figure 5-6: MSD calibration curve for sampling phosgene in air 233

Figure 5-7: 40 L Teflon CFMR chamber for continuous flow, surface-bound permethrin oxidation experiments.

Figure 5-8: Deposition model for reactive gas uptake on indoor surfaces with estimated permethrin reactivity indicated. 
Figure 5A-1: Schematics of (a) Field and Laboratory Emission Cell (FLEC) and

(b) FLEC experimental configuration for permethrin reaction experiments.......245

Figure 5A-2: Ozone reactivity and byproducts of permethrin as tested using a

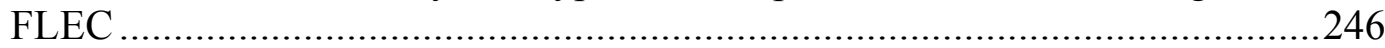

Figure 5A-3: GC-ECD chromatograms from the batch reactor experiments ...........247

Figure 5A-4: Ozone reactivity and byproducts of permethrin as tested using a

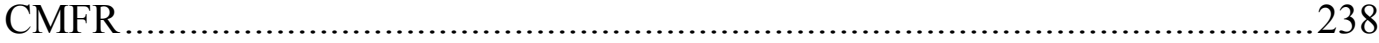




\section{Introduction}

This dissertation explores the chemical and physical factors that affect the transformation of ozone into other airborne pollutants in indoor environments. Ozone reactions and byproduct formation are examined in human-occupied spaces such as residential and commercial buildings and aircraft cabins. The processes of ozone deposition and byproduct formation were measured and modeled for several systems, and the findings are related to potential inhalation exposure.

\subsection{Background: Indoor air quality and ozone chemistry}

"Indoors" encompasses an enormous range of diverse environments from buildings to transportation compartments. Residential buildings range from small apartments to single-family homes to multi-family dwellings. Commercial buildings also vary widely, from theatres to bars to offices. Transportation compartments include passenger vehicle cabins for automobiles, buses, trains, and aircraft. One thing all of these spaces have in common is that they must be ventilated with ambient air. This connection between outside air and inside air has important implications for the indoor environment. In this dissertation, the effect of ozone, an ambient air pollutant, on indoor air quality is explored.

Although indoor spaces vary widely, characteristics common to most environments make it possible to parameterize air quality conditions. A central goal of this dissertation is to characterize the dependence of ozone-related indoor air quality on the important governing factors of indoor environments such as air exchange rate, surface-to-volume ratio, and characteristics of indoor reactive compounds and surfaces. 
Air-exchange rate, as used here, is a first-order coefficient parameterizing the rate at which indoor air is exchanged with outdoor air. For example, an air-exchange rate of 0.2 $\mathrm{h}^{-1}$ indicates that outdoor air will in one hour replace approximately $20 \%$ of the indoor air contained in the space. The full volume of a space would be replaced approximately 3 times during an hour for an air-exchange rate of $3 \mathrm{~h}^{-1}$. Residential and commercial settings have similar air-exchange rates but different means of ventilation; homes are typically ventilated by a combination of infiltration and natural ventilation, and commercial buildings are commonly mechanically ventilated. Occupancy depends on the use of the space - a movie theatre is designed for much higher occupant density than an office setting, for example. Transportation compartments tend to have higher air exchange rates and higher surface-to-volume ratios than buildings, and often have very high occupant densities as well.

There are several compelling reasons why exposure to air pollution in indoor environments can outweigh exposure in outdoor environments. In indoor environments, occupants are in close proximity to sources of pollution. Indoor environments tend to have much lower per-person ventilation rates as compared with atmospheric air sheds. The consequence of this is that - per mass emitted - a pollutant emitted in the indoor environment is much more likely to be inhaled than one emitted outdoors (Smith 1988; Nazaroff, 2008). Because of the indoor sources, the concentrations of some pollutants are higher in the indoor environment than in the outdoor environment, and there are pollutants that exist only or predominantly indoors (Weschler, 2006).

Even when a pollutant is present at a lower concentration indoors than outdoors, a significant fraction of exposure may occur indoors because the average person in the U.S. 
spends the majority of his or her time in indoor environments. A study known as the National Human Activity Patterns Survey found that, on average, people spent a total of $87 \%$ of their time indoors, and $69 \%$ of their time in a residence (Klepeis et al., 2001). Exposure, which is the pollutant concentration times the duration spent exposed to that concentration, could be higher indoors than outdoors on a daily average basis even if pollutant levels are lower indoors, owing to the long times spent indoors. Consider, for example, the case of ozone, which is a reactive pollutant with mainly outdoor sources. Ozone levels indoors are typically $10-50 \%$ of ambient levels because of reactions with gas-phase species and surfaces in the indoor environment. Despite lower levels indoors, Weschler (2006) has estimated that on average $45-75 \%$ of ozone exposure occurs indoors for different U.S. settings. Inhalation intake, which is exposure times breathing rate, is an indicator of dose because it accounts for breathing rate, and thus the amount of pollutant actually inhaled. The estimated fraction of indoor ozone inhalation intake is smaller than exposure, $25-60 \%$ owing to higher breathing rates during outdoor activities (Weschler, 2006). These estimates show that exposure to ozone can be significant indoors. Furthermore, it is also important to consider that the ozone that was consumed in reactions in an indoor environment may generate reaction byproducts.

One class of pollutants for which indoor exposure is expected to be much greater than outdoor exposure is the oxidation byproducts of ozone-initiated chemistry. Ozonereactive compounds are ubiquitous in the gas-phase and on surfaces in the indoor environment. In comparison to the ambient environment, airborne levels of ozonereactive compounds can be much higher indoors because of the use of consumer products, which introduce ozone-reactive species into the air and onto surfaces (Nazaroff 
and Weschler, 2004). In addition, some surfaces are inherently reactive with ozone and others are treated with ozone-reactive compounds in the manufacturing process. Surfaceto-volume ratios are $\sim 100 \times$ higher indoors that outdoors (Nazaroff et al., 2003); thus, oxidation byproducts are likely to be present at higher levels indoors than in outdoor air.

In the indoor environment, ozone reactions in the gas-phase must occur on a time scale at least comparable to that of air exchange to be relevant. However, ozone reactions with surfaces or compounds sorbed to surfaces can have reaction rates slower than the air-exchange rate and still be important ozone sinks and byproduct sources. Weschler (2006) nicely summarized the state of knowledge regarding indoor ozone chemistry with the following points. Reactions with surfaces are an ever-present, dominant sink for ozone and a source of oxidation byproducts in the indoor environment. In addition, episodic releases of highly reactive vapors can be a significant, intermittent sink of ozone and an additional source of byproducts.

There are some indoor sources of ozone including office equipment such as photocopiers and laser printers, electrostatic air cleaners that create ozone as a byproduct, and ozone generators marketed as air cleaners (Weschler, 2000). However, the most common source of ozone in indoor environments is ventilation from outdoors via an air handling unit (i.e. air conditioning), a designed opening (i.e. a window), or through leaks in the building envelope. Thus elevated tropospheric ozone levels affect both outdoor and indoor environments. At ground level, ozone is formed as a consequence of photochemical reactions between $\mathrm{NO}_{\mathrm{x}}$ and reactive volatile organic compounds (VOCs) (Haagen-Smit, 1952; Atkinson, 2000). These precursors have natural sources, so a low level of ozone is present even in remote locations. Elevated levels occur in areas 
proximate to anthropogenic precursor emissions. This effect is amplified in certain urban areas that have low ventilation rates and ample sunlight. Ozone is a criteria pollutant and The National Ambient Air Quality Standard ozone standard is $75 \mathrm{ppb}$ for an 8-hour period and $120 \mathrm{ppb}$ for a 1-h period. Ozone levels are generally highest during daytime in summer, and in polluted conditions can reach low hundreds of ppb ( 100-300 ppb). Typical background or remote ozone levels are in the low tens of ppb ( 10-30 ppb). In the northern hemisphere, ozone concentrations are typically higher than in the southern hemisphere and background levels are increasing (Parrish et al., 2008).

On the ground, ambient ozone levels are dependent on climate and meteorological conditions, geographic features, and proximity to anthropogenic and biogenic sources of ozone precursors. Thus, ozone levels vary significantly by location, and within a location levels vary daily and seasonally. Indoor ozone levels track outdoor ozone levels, but indoor levels are lower owing to reactions with compounds in the air and on surfaces (Weschler, 2000). The ratio of indoor-to-outdoor ozone depends predominantly on the concentration and reactivity of ozone-reactive species in the space and the air-exchange rate for the space. Indoor-to-outdoor ratios for residential and commercial buildings are typically in the range of $0.1-0.5$ (Weschler, 2000), which means that $50 \%$ to $90 \%$ of ozone that enters a building is consumed in reactions.

In the aircraft cabin, the only source of ozone is ventilation of the cabin with air outside the plane, which may contain ozone of stratospheric origin. The air of the upper troposphere and lower stratosphere, where planes fly, is virtually free of reactive air pollutants, except for potentially elevated levels of ozone. The height of the tropopause, which is indicative of the level at which ozone levels start to increase with increasing 
altitude, varies with latitude and season but on average ranges from $8 \mathrm{~km}$ at the poles to $18 \mathrm{~km}$ at the equator (Seinfeld and Pandis, 1998); the tropopause overlaps the typical range of aircraft cruising altitudes $(9-12 \mathrm{~km})$. At cruising altitude ozone levels can rise to hundreds of ppb ( 100-500 ppb), dependent on the flight level relative to the tropopause height (Law et al., 2000). Recent in-flight measurements of ozone on planes indicate that ozone levels in aircraft cabins can range from tens to low-hundreds of ppb ( 10-200 ppb) (Spengler et al., 2004; Bhangar et al., 2008), especially in planes not equipped with ozone control devices.

The reaction of ozone with alkenes in the gas-phase is widely studied (Atkinson, 2000). In general, ozone reacts with the carbon-carbon double bond of unsaturated compounds (Atkinson and Carter, 1984). Immediately after ozone reacts, an ozonide forms that rapidly cleaves to a primary carbonyl (generally an aldehyde or a ketone) and a Criegee biradical. The biradical undergoes further reaction to form a secondary carbonyl (usually an aldehyde, ketone, or carboxylic acid). In the case that a precursor has more than one unsaturation, ozone may also react with the byproducts of the primary ozone-precursor reaction. Ozone reactions with alkenes are also chain-initiating in that the hydroxyl radical $(\mathrm{OH})$ is formed and continues to react and be regenerated (Atkinson and Arey, 2003). The $\mathrm{OH}$ radical is several orders of magnitude more reactive with double bonds (or unsaturations) than ozone, and it is a much less selective oxidizer than ozone, which means that it is capable of reacting with a much larger variety of compounds and functional groups that do not contain double bonds, such as aldehydes (Grosjean and Grosjean, 1997). Therefore, once $\mathrm{OH}$ is formed it may react with the precursor and potentially also with the byproducts of ozone reactions. The term "ozone- 
initiated chemistry" encompasses the primary reaction of ozone with the precursor and the secondary reactions including $\mathrm{OH}$ reaction with the precursor and ozone and $\mathrm{OH}$ reactions with the byproducts.

Ozone-reactive compounds can be in the gas-phase, sorbed on surfaces, or an integral part of a surface material. Ozone reactions on surfaces are less well understood than ozone reactions in the air. Two classes of ozone-reactive compounds are known to be important in indoor environments: terpenes and unsaturated fatty acids. Strictly, the term "terpene" refers to a chemical compound that contains $n$ isoprene units $\left(\mathrm{C}_{5} \mathrm{H}_{8}\right)$ where $n$ is 2 or more, but the term is also used to describe these and closely related chemicals that are otherwise called terpenoids. In this dissertation, "terpene" will be used in its broader sense, referring to terpenes and terpenoids. Essential oils from plants are predominantly terpenes. Terpenes generally have a pleasant scent and good solvent properties. For these reasons, terpenes are common scenting agents and active ingredients in consumer goods such as cleaning products, air fresheners, and personal hygiene products (e.g. shampoo, soap, lotion) (Nazaroff and Weschler, 2004).

Limonene is one of the most commonly found terpenes in consumer products. It is the citrus-scented essential oil that can be found in the peels of oranges, as well as other fruits and plants. It can also be directly emitted from some types of wood furnishings. Limonene is a monoterpene, which means that it has two isoprene units, each with an unsaturation. Ozone rapidly reacts with limonene, forming gas phase byproducts such as formaldehyde. Limonene-ozone reactions also have a very high potential to form secondary organic aerosol (SOA) (Destaillats et al, 2006a). Most terpenes are relatively 
volatile but some terpenes have been found to sorb to surfaces, and products that contain terpenes may be sprayed into the air or applied to surfaces.

As with terpenes, fatty acids are directly produced or derived from biogenic sources. The common sources of fatty acids in indoor environments include human skin oil and skin oil residue, furniture polishes, carpets, linoleum flooring, cooking, and vegetable oils and plant waxes, which may build up on ventilation ducts or filters (Weschler, 2006). Personal hygiene products may also contain unsaturated fatty acids (Pandrangi and Morrison, 2008). Fatty acids are larger molecules that tend to remain condensed on or sorb to surfaces and typically have one or two double bonds when unsaturated.

Recent studies of ozone reactions in the aircraft cabin environment, an indoor environment with high occupant density, have elucidated the importance of human skin oil as a substrate for ozone reactions. Skin oil is a complex combination of squalene, fatty acids, triglyceride fatty acids, free fatty acids, and wax and cholesterol esters (Greene et al., 1970; Nicolaides, 1974). Triglyceride fatty acids are composed of three fatty acids connected to a glycerol molecule, and they are reactive with ozone if they are unsaturated. Squalene is a triterpene, which means that it is composed of six isoprene units. Owing to its six double bonds, it and its byproducts are highly reactive with ozone. Skin oil is readily transferable to other surfaces, and skin oil residue has been used as a surrogate for human subjects to study ozone-surface reactions, including one of the studies presented in this dissertation. The reactions of ozone with skin-oil components are expected to be important for exposure since these reactions may occur very near the breathing zone. 
The reaction of ozone with unsaturated compounds produces potentially odorous or irritating compounds that can be more harmful than the precursors (Weschler, 2004; Weschler, 2006; Wolkoff et al., 2006). The most commonly detected products of ozoneinitiated chemistry include carboxylic acids, aldehydes, ketones, the hydoxyl radical, and secondary organic aerosol (Weschler, 2006; Lee et al., 2006). Saturated and unsaturated aliphatic aldehydes, e.g. nonanal and 2-nonenal, have low odor thresholds and are generally regarded as unpleasant smelling (Uhde and Salthammer, 2007). Odors contribute to degradation in productivity or mood (Wolkoff et al., 2006), and studies have confirmed that perceived air quality can be degraded by the presence of ozone-initiated oxidation byproducts (Wolkoff et al., 2006). Common ozone byproducts are also known carcinogens or respiratory irritants. For instance, formaldehyde and acetaldehyde are classified as probable human carcinogens (IRIS, www.epa.gov/iris/). Other low molecular weight carbonyls - acetic and formic acids - are strong mucous membrane irritants (OSHA, 2008a, 2008b). Ozone-initiated chemistry with alkenes, especially terpenes, may also lead to the formation of oxygenated byproducts that nucleate or condense to form secondary organic aerosol (SOA). Owing to the formation and growth mechanisms of SOA, these particles are small, typically less than $2 \mu \mathrm{m}$ in diameter, and formed of oxygenated organic material. Associations have been established between particle inhalation and adverse health outcomes ranging from respiratory symptoms to mortality, and current evidence indicates that ultrafine $(<0.1 \mu \mathrm{m})$ and fine $(0.1$ to $2 \mu \mathrm{m})$ particles have more serious health effects (Pope and Dockery, 2006). The mechanisms causing particle-related illness, the effects of particle size and composition on health 
effects, and the influence of exposure concentration and duration on health outcome, are all under active investigation as major air-pollution health concerns.

In tests where subjects are exposed to ozone and reactive VOCs, the resulting irritation is ranked higher than the irritation expected from residual precursors or detectable reaction products (Wolkoff et al., 2006). One explanation for this finding is that some species formed from ozone reactions are not currently detected, and these species cause irritation. Based on studies of ozone reaction mechanisms, it is expected that so-called "stealth" compounds are formed in addition to the products that we measure. Stealth compounds may be "short-lived, highly reactive, thermally labile, or highly oxidized" (Weschler, 2006). Techniques have recently been developed to detect one such stealth compound called 4-oxopentanal in indoor settings. 4-Oxopentanal is an oxidation product of ozone and human skin oil. It belongs to a class of compounds called dicarbonyls, which are compounds with two oxygenated functional groups. Low molecular weight carbonyls such as 4-oxopentanal have been found to be sensitizers and to be associated with asthma (Jarvis et al., 2005; Anderson et al., 2007). Current evidence indicates that ozone oxidation byproducts play an important role in the health and comfort of indoor environments, and improved analytical techniques and exposure studies will further elucidate this connection.

In this dissertation, the effects on air quality of ozone reactions with common indoor gas-phase species or surface compounds are investigated. I used a combination of laboratory experiments and modeling to determine ozone uptake by, and byproduct generation from, indoor precursors including cleaning products, aircraft cabin furnishings, worn clothing, and a residual insecticide. I also developed a model of ozone 
reactions with a chemically reactive surface-bound residue. Ozone uptake on surfaces was characterized using deposition velocity and reaction probability, and byproduct generation was characterized for gas- and surface-phase reactions using emission rates and yields. The extent to which environmental factors such as ozone level, precursor level (including surface-to-volume ratio) and precursor type, and air-exchange rate affect byproduct formation were investigated. Results of these studies are useful for predicting the concentrations of ozone-initiated byproducts in indoor settings and relating them to appropriate health endpoints.

\subsection{Introduction to dissertation projects}

Chapter 2 focuses on secondary organic aerosol from ozone-terpene reactions indoors. The formation of secondary organic aerosol (SOA) from the reaction of terpenes with ozone is widely studied in the field of atmospheric chemistry (Odum et al., 1996). In ambient air, ozone is formed in-situ, and terpenes are present as biogenic emissions from vegetation. Terpenes are quite reactive with ozone owing to multiple unsaturated carbon bonds. Ozone and terpenes react to form products, some of which are less volatile than their precursors and condense leading to formation and growth of SOA (Koch et al., 2000). A ubiquitous source of terpenes in the indoor environment is cleaning products and other scented household products such as air fresheners (Nazaroff and Weschler, 2004).

In Chapter 2, I analyze formation and growth dynamics of secondary organic aerosol (SOA) from ozone reactions with terpene-containing consumer products. The particle data were collected in well-controlled, bench-scale chamber experiments. Size- 
and time-resolved aerosol measurements were made over a relatively wide size range (10 $\mathrm{nm}$ to $1.1 \mu \mathrm{m}$ ) for indoor air quality studies and were used to determine particle size distribution characteristics. Analysis techniques commonly employed in studies of atmospheric SOA were employed to analyze factors affecting SOA nucleation and growth dynamics under indoor conditions.

Gas-phase emissions of highly reactive VOCs can have a significant effect on indoor air quality, but their emissions indoors are episodic. The dominant sink of ozone and source of byproducts in indoor environments is reactions on fixed surfaces (Weschler, 2000). Chapter 3 examines ozone reactions with surfaces in the aircraft cabin environment. In the cabin, surface-to-volume ratios are higher than in most buildings, ozone concentrations can be greater than in ground-based environments, and the occupant density of the cabin environment is typically very high. Recent studies of cabin air quality indicate that not only fixed surfaces, but also surfaces associated with passengers, consume a large fraction of ozone entering the cabin and significantly contribute to the formation of gas-phase oxidation byproducts. Chapter 3 describes bench-scale chamber experiments to measure ozone uptake and subsequent byproduct formation from individual cabin surfaces, a yet unexplored area. Contributions of surface categories to overall ozone deposition and byproduct emissions were evaluated and were found to be comparable to studies conducted in a cabin setting.

In Chapter 3, inhalation intake of oxidation byproducts in the cabin environment was also explored. A model was developed to predict byproduct levels in the cabin based on three input parameters: cabin ozone level, byproduct yield, and retention ratio. Distributions for each of these three parameters were determined from this study and 
several other recent studies of cabin air quality. A Monte Carlo method was used to determine the distribution of total byproduct concentrations in the cabin. Inhalation intake rates of a few individual byproducts (formaldehyde, nonanal, and 4-oxopentanal) were calculated for aircraft cabin environments and compared to intakes rates in other environments such as in buildings and outdoors.

Previous studies have modeled ozone consumption on indoor surfaces of uniform reactivity, and that model was employed in Chapter 3. In Chapter 4, that modeling approach is extended to estimate ozone removal with a reactive residue that partially covers a base surface. The model accounts for mass-transport of ozone through the nearsurface concentration boundary layer plus competitive reaction between the base surface material and a chemical residue that covers a fraction of that surface. A method for predicting time-dependent residual consumption and byproduct formation is also developed, given the ozone uptake rate of the residue, residue-specific byproduct yields, and indoor ozone level. The model is illustrated with the example of oleic acid, a common fatty acid, on a relatively nonreactive surface. A parametric investigation of the model is presented. The model is also applied to measurements from chamber experiments of ozone-surface reactions.

In Chapter 5, oxidation of a pesticide containing a halogen-substituted alkene group is explored. A pesticide that is commonly used for residual (long-lasting) insecticide treatment in the aircraft cabin was identified as a potential ozone-reactive compound. The pesticide - permethrin - would, in principle, form phosgene when oxidized by ozone. A derivatization method was developed to detect phosgene at low levels, and laboratory experiments were conducted to verify the reaction. However, in the 
course of the experiments it was determined that chemical attributes of the pesticide slow the reaction with ozone to below detectable limits. Details of the investigation of ozone reactions with the pesticide permethrin are presented. The model of ozone deposition used to extrapolate from chamber experiments to the cabin environment in Chapter 3 is employed to determine the upper-bound on phosgene formation in the cabin in Chapter 5. A summary of the chapters and suggestions for future research are given in Chapter 6. 


\section{Secondary organic aerosol from ozone reactions with cleaning products}

Reproduced in part with permission from Atmospheric Environment 42, 8234-8245, 2008. Copyright 2008, Elsevier Inc.

\subsection{Introduction}

Terpenes and ozone are commonly present indoors and their reactions can produce particles (Weschler and Shields, 1999; Long et al., 2000; Wainman et al., 2000; Sarwar et al., 2004; Liu et al., 2004). Many consumer products, such as cleaning agents and air fresheners, are sources of indoor terpenes (Nazaroff and Weschler, 2004). Ozone is routinely present indoors because of ventilation with ozone-containing outdoor air (Weschler, 2000).

Inhaling particles raises health concerns (Pope and Dockery, 2006). Deposition in the lungs is size-dependent (Yeh et al., 1996; Asgharian and Price, 2007), and the health effects associated with aerosol exposure depend on particle size and concentration (Oberdörster, 2001; Peters et al., 1997). The exposure impacts of indoor pollutants are amplified because (a) people spend a high proportion of their time indoors, (b) emissions that occur indoors are diluted into confined volumes and removed at slow ventilation rates, and (c) people tend to be in close proximity to indoor sources (Nazaroff, 2008). Consequently, it is important to characterize both the source strength and size distribution of significant indoor particle sources. 
Secondary organic aerosol (SOA) formation from ozone-terpene interactions has been widely studied in relation to atmospheric organic aerosol formation. Because of the different conditions outdoors and indoors, those studies have uncertain direct applicability for elucidating SOA formation indoors. Several studies have measured SOA formation and growth from ozone reactions with pure terpenes or with terpene-containing products under indoor-relevant conditions. Most of these studies measured particles using an optical particle counter (Weschler and Shields, 1999; Wainman et al., 2000; Weschler and Shields, 2003; Sarwar et al., 2003; Sarwar et al., 2004; Hubbard et al., 2005; Singer et al., 2006a). Optical particle counters (OPC) typically measure only particles that are $100 \mathrm{~nm}$ or larger and thus cannot characterize the ultrafine particles that are an essential component of particle nucleation and growth. A few studies have characterized indoor ultrafine particles using a scanning mobility particle sizer (SMPS) to investigate nucleation and growth in these cases: (a) use of a pine-oil based cleaner in the presence of ozone (Long et al., 2000); (b) peeling of oranges in the presence of ozone (Vartiainen et al., 2006); (c) adding limonene to an office environment in which an ionizing air purifier was used (Alshawa et al., 2007); and (d) reacting limonene and ozone in a large chamber (Zuraimi et al., 2007; Langer et al., 2008).

In the present study, particle formation and size-distribution dynamics are investigated using SMPS and OPC data for experiments in a small, flow-through chamber. Ozone was reacted with vapor emissions of terpene-containing consumer products (two cleaning products and an air freshener) at indoor-relevant conditions. Destaillats et al. (2006a) reported on the consumption of primary constituents and the formation of secondary products from these experiments, emphasizing gaseous species. 
Here, data from the same experiments are presented in detail and analyzed to characterize particle size distributions. The effects on SOA production of factors such as chamber airexchange rate and ozone level are investigated. Particle formation and growth dynamics are analyzed, and the particle mass formation rate is modeled.

\subsection{Experimental conditions}

Experiments were conducted in a Teflon-lined, 198-L rectangular chamber with a surface-to-volume ratio of $10 \mathrm{~m}^{-1}$. Details of the experimental apparatus and gas-phase chemical analyses are presented in Destaillats et al. (2006a). Briefly, cleaning product vapor was continuously introduced into the chamber and, after steady-state was reached, continuous ozone addition commenced. Figure 2-1 illustrates the chamber configuration. The cleaning product vapor was introduced through Teflon tubing in one bottom corner of the chamber while ozone was introduced through Teflon tubing in the diagonal opposite bottom corner. Particles were sampled at the middle of the chamber ceiling. Experiments were performed at $23.0 \pm 0.5^{\circ} \mathrm{C}$.

Three cleaning products were tested: a pine oil cleaner (POC); an orange-oil degreaser (OOD); and a heated, scented-oil air freshener (AFR). Key reactive constituents likely to contribute to SOA formation are listed here; detailed composition of the products is reported in Singer et al. (2006b). The OOD contained only one terpene, dlimonene. The POC contained several volatile and reactive constituents, including dlimonene, terpinolene, $\alpha$-terpinene and $\alpha$-terpineol. The AFR was the most complex mixture, with more than 30 volatile terpenes, terpenoids, and other compounds, the most reactive of which were d-limonene, linalool, dihydromyrcenol, $\beta$-citronellol, and linalyl 
acetate. A summary of the composition data for terpenes (supply and residual levels) is given in an appendix, Section 2.B.

For each product, three experiments were conducted with two ozone supply levels and two air-exchange rates (AERs) in the following configurations: $130 \mathrm{ppb}, 3 \mathrm{~h}^{-1}$ (denoted HH); $60 \mathrm{ppb}, 3 \mathrm{~h}^{-1}$ (denoted MH); and $130 \mathrm{ppb}, 1 \mathrm{~h}^{-1}$ (denoted HL). Seven additional experiments with the POC were performed. Experimental conditions are summarized in Table 2-1. The total cleaning product constituent levels were similar for each of the three configurations and the total amount of reactive terpenes and terpenoids in the inflow were also similar for each cleaning product, approximately $700 \mathrm{ppb}$. All experiments, unless otherwise stated, were performed with zero grade air (Airgas) humidified by means of a sparger to $50 \%$ relative humidity (RH). The zero air was virtually particle free, but flowing the air through the sparger containing pellets of activated carbon ( $\sim 0.5 \mathrm{~cm}$ in diameter) inadvertently introduced "seed" particles. With the sparger in use, the supply air contained a particle concentration of $\sim 400 \mathrm{~cm}^{-3}$. The geometric median diameter was $\sim 30 \mathrm{~nm}$, the geometric standard deviation was 1.3 , and the mass concentration was $\sim 0.005 \mu \mathrm{g} \mathrm{m}^{-3}$.

With the pine-oil cleaner, the range of ozone supply levels was extended (POCVH and POC-LH) and a replicate experiment was conducted (POC-HH1 and POC-HH2). Four supplemental experiments were also conducted. In POC-Rev, the reagents were added in reverse order, i.e. the cleaning product vapor was introduced into the chamber that already contained a steady-state level of ozone. In POC-NOx, steady-state levels of $74 \mathrm{ppb}$ of $\mathrm{NO}_{2}$ and $1.75 \mathrm{ppb}$ of $\mathrm{NO}$ were present in the chamber, together with the VOC mixture and before addition of ozone, to explore the effect of the nitrate radical on 
oxidative chemistry. In POC-Seed, laboratory air was used instead of zero air to investigate the effects of a more realistic atmospheric seed particle distribution on secondary product formation. The POC-NOx, POC-Rev, POC-Seed experiments were performed at an AER of $3 \mathrm{~h}^{-1}$ and $\sim 130 \mathrm{ppb}$ supply ozone. To investigate the effect of water vapor, a POC-Dry experiment was performed using zero-air without any humidification at $3 \mathrm{~h}^{-1}$ and $\sim 60 \mathrm{ppb}$ of ozone in supply air.

Tracer gas tests confirm that the chamber was well mixed (Destaillats et al., 2006a). A comparison of estimated characteristic times for mixing and reaction indicates that these two processes have similar time scales. Particles were sampled from only one position in the chamber. Some features of the particle data might have been influenced by spatial variability.

\subsection{Particle measurement and analysis}

\subsubsection{Instruments}

Aerosol size distribution measurements were performed using a scanning mobility particle sizer (SMPS) in every experiment and an optical particle counter (OPC) in some experiments. The SMPS measured particles in the diameter range $0.008-0.415 \mu \mathrm{m}$ in 64 bins; data from 0.01-0.4 $\mu \mathrm{m}$ were used for analysis. The SMPS consists of a differential mobility analyzer (3701A, TSI Inc.) and a condensation particle counter (3760, TSI Inc.). The SMPS performed a complete scan (up and down the size distribution) approximately every $2 \mathrm{~min}$. The data were collected and inverted using the Labview interface with software written by D Collins (Texas A\&M University) and P Chuang (UCSC) and analyzed using Igor (Wavemetrics Inc.) with custom routines. The OPC (Lasair 1003, Particle Measuring Systems, Inc.) measured 0.1-2 $\mu \mathrm{m}$ diameter particles in eight bins. 
For both instruments, particle volume concentration was estimated by multiplying the measured number concentration by $\left(\pi / 6 \times \mathrm{GMD}^{3}\right)$, where GMD, the geometric mean diameter of a bin, is the square root of the product of the upper and lower bin diameters. A particle density of $1 \mathrm{~g} \mathrm{~cm}^{-3}$ was assumed in converting volume to mass; this may underestimate particle mass concentration as some studies have reported the density of organic atmospheric particles to be 1.2 to $1.5 \mathrm{~g} \mathrm{~cm}^{-3}$ (Khlystov et al., 2004; Turpin and Lim, 2001).

The OPC sampled for 1 minute every 2 minutes, counting particles in eight bins simultaneously. The lower bin bounds, as calibrated by the manufacturer with polystyrene latex (PSL) particles, were $0.1,0.2,0.3,0.4,0.5,0.7,1.0$, and $2.0 \mu \mathrm{m}$. However, the instrument's response is influenced by a particle's refractive index, $m$, which depends on its chemical composition. The refractive index of PSL is 1.588. Organic particles tend to have a lower refractive index (Dick et al., 2007). Accurately sizing particles with a different refractive index requires scaling the bin bounds. For instance, a $0.15 \mu \mathrm{m}$ particle of oleic acid $(m=1.46)$ would be sized as a $0.1 \mu \mathrm{m}$ particle in an OPC calibrated using PSL (Hand and Kreidenweis, 2002). The OPC counts the number of particles in each bin and must be divided by the log of the difference in the upper and lower bin bounds $\left(\log \left(D_{p, \text { upper }}-D_{p, \text { lower }}\right)\right)$, and by the sample volume $\left(14.2 \mathrm{~cm}^{3}\right)$, to get the number size distribution and then multiplied by $\pi / 6 \times \mathrm{GMD}^{3}$ to determine mass size distribution. Thus bin size affects both the number and volume (or mass) size distributions. 


\subsubsection{Alignment routine}

Hand and Kreidenweis (2002) calibrated the same model of OPC as used in this study with PSL ( $m=1.588)$, dry ammonium sulfate $(m=1.53)$, and oleic acid $(m=1.46)$. From those data, they developed polynomials for scaling the manufacturer's bin bounds. In the present study, a routine in the Igor programming language was developed that employed these scaling polynomials in aligning the data collected where the size range measured by the OPC and SMPS overlap. The code is presented in an appendix (Section 2.A.). At each time step, using increments of $\Delta m=0.01$, the alignment routine scanned the range of possible $m$ values from 1.46 to 1.59 , calculated OPC bin bounds based on $m$, summed the SMPS number concentrations within those bounds, and compared the results to the measured OPC number concentration. The procedure yielded a time-dependent $m$ value that produced optimal alignment between the OPC and SMPS data, determined using the least-squares difference between the SMPS and adjusted OPC concentrations. Only two or three size bins of the OPC overlapped with the SMPS data. The refractive index determined from this routine is not intended to be a robust measure of the refractive index of the particles. Instead, the purpose was to determine an appropriate adjustment of the OPC bin bounds to improve estimates of particle mass concentrations.

The results matched our expectation that SOA has a refractive index similar to that of oleic acid; the alignment routine indicated $m=1.46-1.49$. The OPC bins were scaled for oleic acid, and the lower bin bounds used were $0.15,0.24,0.36,0.47,0.62$, 0.89 , and $1.1 \mu \mathrm{m}$ (the eighth bin was not modeled). Data from the first six bins, which had both lower and upper bounds, were used to calculate particle size distributions and total number and mass. Thus, total mass concentrations given in this paper can be 
represented as $\mathrm{PM}_{1.1}$. Particles were measured in all eight bins. No particles were measured in the eighth bin for any experiment. The particle number concentration in the seventh bin was very low, $<1 \mathrm{~cm}^{-3}$, for most experiments except POC-VH where the maximum particle number concentration in the seventh bin reached $\sim 7 \mathrm{~cm}^{-3}$. Assuming this bin has a GMD of $2.0 \mu \mathrm{m}$, a particle number concentration of $7 \mathrm{~cm}^{-3}$ would contribute $\sim 30 \mu \mathrm{m}^{3} \mathrm{~cm}^{-3}$ to the total particle volume (or $\sim 30 \mu \mathrm{g} \mathrm{m}^{-3}$ to the total particle mass assuming unit particle density), which would contribute an extra $\sim 15 \%$ to the total volume measured in POC-VH.

\subsubsection{Wall losses}

The pseudo first-order rate coefficient for particle deposition to chamber surfaces, $L_{d e p}$, was determined using equation 2-1 at each particle scan.

$$
L_{d e p}=\int_{D_{p, \text { min }}}^{D_{p, \text { max }}}\left(\beta_{\text {dep }}\left(D_{p}\right)\right)\left(\frac{d N}{d \log D_{p}}\right) d \log D_{p}
$$

Here, $N$ is particle count $\left(\mathrm{cm}^{-3}\right), D_{p}$ is particle diameter $(\mu \mathrm{m}$ or $\mathrm{nm}), D_{p, \min }$ is the minimum particle size ( $10 \mathrm{~nm}$ for the SMPS), and $D_{p, \max }$ is the maximum particle size (1.1 $\mu \mathrm{m}$ for the OPC). The size-dependent deposition loss-rate coefficients, $\beta_{\text {dep }}$, were estimated using the model of Lai and Nazaroff (2000). This model requires the input of a friction velocity to characterize near-surface flows. The actual friction velocity in the chamber was unknown, so a range of plausible values was used. The calculated characteristic time for deposition was very long compared to removal by ventilation. For example, in experiment POC-MH, the minimum characteristic time for surface deposition (which occurs at the peak number concentration) was 70 to $700 \mathrm{~h}$, respectively, for friction velocities of 3 to $0.3 \mathrm{~cm} \mathrm{~s}^{-1}$. The characteristic time for particle loss by ventilation 
was $0.3 \mathrm{~h}$. Consequently, I concluded that particle deposition to chamber surfaces could be neglected in further analysis.

Characteristic times for deposition of condensable vapors to walls were also calculated using the Lai and Nazaroff (2000) model, incorporating a plausible range, 0.04-0.08 $\mathrm{cm}^{2} \mathrm{~s}^{-1}$, for the diffusion coefficients of the semivolatile products of terpene oxidation. With a minimal diffusion coefficient of $0.04 \mathrm{~cm}^{2} \mathrm{~s}^{-1}$ and the same range of friction velocities used to calculate particle deposition ( 3 to $0.3 \mathrm{~cm} \mathrm{~s}^{-1}$ ), the characteristic time for loss of vapors to walls was 200-2000 s. Although this is fast compared to ventilation, it is a relatively small sink compared to vapor condensation onto particles. The first-order condensation rate for vapor onto particles, $L_{\text {cond }}$, was estimated using the Fuchs and Sutugin equation for mass transfer of gas to particles in the transition regime. The condensation coefficient, $\beta_{\text {cond }}$, was calculated using equation 2-2 (equation 11.34 in Seinfeld and Pandis, 1998) and integrated over the particle size range in manner analogous to equation 2-1. The code used to perform this calculation and an example plot of the condensation rate with time are presented in an appendix, Section 2.A.

$$
\beta_{\text {cond }}\left(D_{p}\right)=2 \pi D_{p} \mathcal{D}\left(\frac{1+K n}{1+1.71 K n+1.33 K n^{2}}\right)
$$

The diffusion coefficient of the gas-phase species is $\mathcal{D}$, and $K n$ is the Knudsen number. As an example, in experiment POC-MH, the characteristic time for vapor deposition to particles, assuming a diffusion coefficient of $0.04 \mathrm{~cm}^{2} \mathrm{~s}^{-1}$ was $6 \mathrm{~s}$ just after the initial nucleation event and a maximum of $10 \mathrm{~s}$ for the remainder of the experiment. Over the ranges of particle and airflow conditions and vapor diffusivities in these experiments, the 
time scale for condensation to particles was at least an order of magnitude faster than for vapor deposition onto walls.

\subsubsection{Coagulation sink}

The pseudo first-order rate coefficient for particle loss by coagulation, $L_{\text {coag, }}$, was determined using equation 2-3 for each scanned particle size distribution.

$$
\begin{aligned}
& L_{\text {coag }}^{*}\left(D_{p}^{*}\right)=\int_{D_{p, \text { min }}}^{D_{p, \text { max }}}\left(\beta_{\text {coag }}\left(D_{p}^{*}, D_{p}\right)\right)\left(\frac{d N}{d \log D_{p}}\right) d \log D_{p} \\
& L_{\text {coag }}=\int_{D_{p, \text { min }}}^{D_{p, \text { max }}}\left(L_{c o a g}^{*}\left(D_{p}\right)\right)\left(\frac{d N}{d \log D_{p}}\right) d \log D_{p}
\end{aligned}
$$

The size-dependent loss coefficient, $\beta_{\text {coag }}$, for coagulation of a particle of size $D_{p}{ }^{*}$ to the particle distribution was calculated using the Fuchs form of the Brownian coagulation coefficient as given in Table 12.1 of Seinfeld and Pandis (1998). Compared with removal by ventilation, coagulation was estimated to contribute $\sim 25 \%$ to the total particle number concentration sink immediately after nucleation and $\sim 10 \%$ during later stages of the experiments.

\subsection{SOA formation and growth stages}

SMPS data from several POC experiments are presented in Figure 2-2. Similar particle formation and growth behavior was exhibited in all experiments, and particle data from each experiment is shown in an appendix, Section 2.B. For the present discussion, this behavior is divided into four stages, as illustrated in Figure 2-3 using experiment AFR-HH as an example. Stage 1 is characterized by an initial nucleation burst occurring immediately after ozone is introduced. The dominant feature of stage 2 is 
the growth of the particles formed during the initial burst. Stage 3 occurs after ventilation has removed the majority of the particles originally formed; this stage is characterized by the onset of secondary nucleation events. In stage 4 , all of the original particles have left the system. This stage is referred to as quasi-steady-state because the particle size distribution exhibits persistent time-dependent behavior, but of a repetitive or cyclic character.

\subsection{Size distribution characteristics}

Figure 2-4 shows examples of the particle size distributions from stage 4 for three experiments. Measured and modeled size distributions from each experiment are given in Section 2.B. Figure 2-4a is an example where both the SMPS and OPC data could be combined to produce a good fit. Figure $2-4 \mathrm{~b}$ shows an experiment where almost the entire distribution was within the range of the SMPS. Figure 2-4c shows an experiment where the distribution was outside the range of the SMPS but no OPC data were available. In these cases, the characteristics of the upper size range of the distribution were estimated using typical distribution parameters determined from other experiments.

In experiments where both SMPS and OPC data were collected, lognormal distributions were fit to the combined (SMPS and adjusted OPC) size distributions. Three modes were required to fit the data well; using four modes did not significantly improve the fit. Manual fitting of the lognormal distribution parameters was required because, mathematically, the measured distributions were not well constrained owing to missing data at the tails. Modeled distribution parameters (number concentration, $N$; geometric 
mean diameter, GMD; and geometric standard deviation, GSD) are presented for each mode in Table 2-2.

For experiments without OPC data, the height and central tendency ( $N$ and GMD) for the largest diameter mode could be determined with reasonable confidence from the SMPS data, but the spread (GSD) of the distribution was unknown. Since most fits with both OPC and SMPS data indicated a GSD of 1.4 for the third (largest) mode, this value was used to fit the experiments where only SMPS data were available. Overlaid in each example in Figure 2-4 is the best-fit 3-mode lognormal distribution. The modeled particles mass, $\mathrm{PM}_{1.1}$, and mass that would have been measured by SMPS, $\mathrm{PM}_{0.4}$, were calculated using the manual fit distributions parameters using the code shown in an appendix, Section 2.A.

Table 2-3 presents the measured and modeled particle data for each of the small chamber experiments. Peak total number concentrations during stage 2 were on the order of $10^{5} \mathrm{~cm}^{-3}$ and the particle number concentrations during stage 4 were an order of magnitude lower. Since the vast majority of the particles were smaller than $400 \mathrm{~nm}$, the SMPS provided a fairly accurate measure of the total particle number concentration. Mass concentration $\left(\mathrm{PM}_{1.1}\right)$ ranged from tens to hundreds of $\mu \mathrm{g} \mathrm{m}^{-3}$, and the stage 4 mass concentration was about half of the value at the peak. Together, the SMPS and OPC captured the full range of particle sizes, but up to half of the mass went undetected when only the SMPS was used. 


\subsubsection{Effect of ozone level and air-exchange rate on mass and yield}

Aerosol yield $(Y)$ was calculated as the total mass concentration of SOA $\left(P M_{1.1, S S}\right)$ formed per mass concentration of VOC consumed $(\triangle V O C)$. Integrated gas-phase samples were taken at steady-state before and after ozone addition, and yields were calculated using steady-state particle mass concentration. For the two experiments (OOD-HL and POC-HL) where the size distribution varied significantly during the steady-state period, average $P M_{1.1, S S}$ was used to estimate yield.

As shown in Figure 2-5a, the steady-state mass concentration of SOA was greater at the higher supply ozone level for each of the three products. The relationship between yield and ozone level was similar to the relationship between mass concentration and ozone, although the yield for POC appeared to level off above $~ 100 \mathrm{ppb}$ ozone, as shown in Figure 2-5b. Limonene, $\alpha$-terpinene, and terpinolene have the highest SOA-forming potential of the terpenes in the tested products (Lee et al., 2006; Koch et al., 2000). The total terpene level (in ppb) introduced was roughly constant from one experiment to the next, but the fraction of terpenes with high SOA-forming potential decreased from $100 \%$ in OOD to $65 \%$ in POC to $10 \%$ in AFR. The relative level of SOA generation for each household product roughly corresponds to these ratios.

The yield values are consistent with those reported in other indoor studies. A yield of $10-15 \%$ was estimated for an experiment that introduced a limonene source into an office ventilated with outdoor air that contained a moderate amount of ozone (Weschler and Shields, 1999). A yield of $\sim 25 \%$ was calculated from particle and terpene data collected from a pine-scented heated air freshener in a chamber study with $50 \mathrm{ppb}$ residual ozone (Liu et al., 2004; yields calculated from Figure 7). A yield of 13\% was 
reported for limonene injected into an office in which an air-cleaning device that produces ozone was operated (Alshawa et al., 2007).

Relative to the case for AER $=3 \mathrm{~h}^{-1}$, SOA production appears to decrease for POC and OOD and increase for AFR when AER $=1 \mathrm{~h}^{-1}$ (see Figure 2-5a). However, when the steady-state particle concentration is compared to the mass rate of precursor consumption (Figure 2-5c), it is clear that more SOA is produced per mass of precursor consumed when the air-exchange rate is lowered. This finding is consistent with the expectation that longer reaction times allow for greater oxidation of primary ozone reaction products, and that second generation products can make a significant contribution to SOA production. For instance, the reaction of ozone and limonene is chain-initiating, producing not only first-generation oxidation products but also the hydroxyl radical (Aschmann et al., 2002); ozone and $\mathrm{OH}$ react with the first-generation oxidation products to form second-generation products and so on. Second and subsequent generations of oxidation products can be even less volatile than first-generation products leading to increased SOA mass and yield (Leungsakul et al., 2005; Ng et al., 2006; Zhang et al., 2006). We postulate that in the low AER case, the secondary oxidation products formed from the ozone-limonene reaction have additional time to react, forming even lower volatility products, which tend to condense and increase the total mass of SOA formed. This inference is consistent with the lower ozone and $\mathrm{OH}$ radical concentrations previously reported for the low AER experiments, as compared with those determined for the same conditions, but with higher AER (Destaillats et al., 2006a). The increased time in the chamber may also allow for more oxidation of the slower-reacting compounds, especially in AFR. 


\subsubsection{Effect of $\mathrm{RH}$, order of reagent addition, and ambient seed particles}

The POC-Rev, POC-NOx, and POC-Seed experiments were conducted under the same conditions as POC-HH1 and POC-HH2. Reversing the order of reagent addition in experiment POC-Rev had little effect on initial or steady-state particle characteristics, which can be seen by comparing Figures 2-2a and 2-2d. The addition of NOx to the supply air did not have an evident effect on SOA formation. In POC-Seed, the seed particle number concentration was similar to that in humidified zero-air experiments $\left(\sim 500 \mathrm{~cm}^{-3}\right)$, but the distribution of seed particles was shifted toward larger particles $(\mathrm{GMD}=98 \mathrm{~nm}, \mathrm{GSD}=2.2)$ compared with humidified zero air $(\mathrm{GMD}=30 \mathrm{~nm}, \mathrm{GSD}=$ 1.3), resulting in 100 times greater particle mass concentration in the supply air (0.5 versus $\left.0.005 \mu \mathrm{g} \mathrm{m}^{-3}\right)$. The steady-state particle number and mass concentrations were $2.3 \times$ and $1.8 \times$ higher, respectively, for POC-Seed than POC-HH1.

The POC-Dry experiment was conducted under the same conditions as POC-MH. Only in executing the "dry" experiment, was it discovered that the water sparger used to humidify air was the source of seed particles in all 50\% RH experiments. The number and mass particle concentrations in the supply air for the POC-Dry experiment were $<5$ $\mathrm{cm}^{-3}$ and $<0.001 \mu \mathrm{g} \mathrm{m}^{-3}$, respectively, and corresponding concentrations in POC-MH were $380 \mathrm{~cm}^{-3}(\mathrm{GMD}=24 \mathrm{~nm}, \mathrm{GSD}=1.3)$ and $0.005 \mu \mathrm{g} \mathrm{m}{ }^{-3}$. The effects of removing the seed particles and lowering the RH cannot be separated in our experiments; the overall effect was to lower the particle number and mass concentration (see Figures 2-2b and 2-2c). Cocker et al. (2001) reported that moderate RH tended to increase the overall aerosol yield owing to the hygroscopicity of aerosol-phase organic material, whereas aqueous seed particles containing salts would tend to lower the overall aerosol yield 
owing to interactions between the salts and the organic material. The seed particles in our experiments were likely aqueous, but it is unknown whether they contained salts.

\subsection{Particle formation and growth characteristics}

\subsubsection{Cycle of particle formation and growth}

Figure 2-2f shows experimental results in which a repeated cycle of particle formation and growth occurs in stages 3 and 4 . The balance between new particle formation and growth of existing particles in the chamber can be plausibly explained as follows. As particles grow by condensation, they are also are removed by ventilation, reducing the total surface area available for condensation. Condensable vapor then accumulates until a burst of nucleation occurs that significantly lowers the vapor concentration. These newly formed particles grow while continuing to be removed from the chamber by ventilation, again reducing the surface area available for condensation, and the cycle repeats. The balance between nucleation and growth is most clearly visible in the low AER experiments, as illustrated by the repeated appearance of plumes in Figure 2-2f for experiment POC-HL. In contrast, in the higher AER experiments, particles are so rapidly ventilated out of the system that new particle formation by nucleation seems to occur continuously. The plumes of these nucleation events are compressed to a timescale similar to that of the SMPS measurements, giving the appearance of a steady-state particle size distribution. An example of this behavior is illustrated in Figure 2-2a for experiment POC-HH. Experiment POC-MH exhibited behavior between these two extremes, as shown in Figure 2-2b. Trends in particle mass and number concentration support this description. In experiments where the particle behavior is dynamic, such as in POC-HL (Figure 2-2f) and OOD-HL, the mass 
concentration and GMD increase and the number concentration decreases with time after each nucleation event (see Section 2.B.).

This process of condensable vapor accumulation and subsequent nucleation burst is also thought to be responsible for the thin plumes that appear along with the main plume in stage 2 of every experiment. Once the initial burst of particles is formed, some particles are removed by ventilation. Condensable vapor accumulates that cannot be accommodated by condensation onto the existing particles and a small new burst of nucleation occurs, which creates a new peak in the size distribution. Condensational growth on these new particles shifts the peak toward larger particles sizes over time generating the thin plume seen in the SMPS data.

The rate of decay of particle number concentration in the chamber following the initial burst provides additional evidence of persistent particle nucleation. After the initial nucleation burst, the particle number concentration decays more slowly than expected from ventilation alone. Figure 2-6 compares the observed pseudo first-order loss rate of particle number concentration with the air-exchange rate for experiment POCMH. The apparent loss rate was $2.5 \mathrm{~h}^{-1}$ while the AER was $3.0 \mathrm{~h}^{-1}$. From this information, we infer that nucleation must be occurring to provide a fresh source of new particles that offsets some of the removal by ventilation. In this experiment, about 40 minutes after ozone was introduced (at 16:25), the particle loss rate slowed, and then rebounded slightly and settled at an effectively constant level. The inflection corresponds to the onset of stage 3, with the occurrence of distinct new nucleation events, as can be seen in Figure 2-2b. New particle formation occurred because not enough of the original particles remained in the chamber to accommodate the condensable vapor being formed. The dip 
in the particle number concentration is the result of two effects. First, the originally created particles have grown so large that they are no longer counted by the SMPS. Second, so long as the large particles from the initial burst remain, their growth in surface area means that fewer particles are required to accommodate the flow of condensable mass as time progresses.

The balance between condensation and nucleation is also evident in the OPC data. As shown in Figure 2-3, the number concentration of particles in the $0.62-0.89 \mu \mathrm{m}$ size bin stagnates as secondary nucleation starts with the onset of stage 3. Stagnation occurs because the condensational growth is now apportioned between the numerous newly formed particles and the small number of residual large particles. Temporary stagnation in this size bin at the onset of secondary nucleation is seen in all experiments for which OPC data were collected, and the effect is more pronounced for experiments with higher particle concentrations.

\subsubsection{Nucleation subsequent to the initial event}

The nucleation events subsequent to the initial burst do not produce the large number concentrations of particles that were created in the initial event. There are two likely reasons for this depressed nucleation intensity. First, there is not as much condensable material in the system in stages two through four as there was when ozone was first introduced. When ozone first enters the chamber, the concentration of terpenes in the system is at its highest; for the remainder of each experiment, ozone only can react with the residual terpene concentrations. Second, there are many preexisting particles in 
the system after the initial burst, and some of the condensable material contributes to particle growth rather than to nucleation.

The inference that nucleation is occurring in stages 3 and 4, rather than just growth of seed particles, is reinforced by two observations: (1) the particles present in the third and fourth stages are smaller than those present in the supply air; and (2) the number concentration of particles in the chamber persists at a level that is orders of magnitude higher than the number concentration of particles in the supply air. It is very unlikely that so many seed particles smaller than the lower limit of the SMPS $(\sim 8 \mathrm{~nm})$ would exist in the supply air and could therefore be responsible for the appearance of smaller particles in the system through condensational growth.

At steady state, the particle nucleation rate can be evaluated from a number balance. The only significant source contributing to the total particle number concentration in the chamber, $P_{N}$, is nucleation, and the dominant sink is removal by ventilation, so a material balance is:

$$
\frac{d P_{N}}{d t}=R_{N}-\lambda P_{N} \quad R_{N}=\lambda P_{N, S S}
$$

At steady state, the rate of nucleation, $R_{N}$, can be estimated as the chamber air-exchange rate, $\lambda$, times the steady-state particle number concentration, $P_{N, S S}$. The air supply is not included as a particle source as the number of particles in the supply air is negligible compared to that when ozone and terpenes are present. For example, in experiment POC$\mathrm{MH}$, the supply air contained a particle concentration of $350 \mathrm{~cm}^{-3}$, whereas the stage 4 chamber concentration was $24,000 \mathrm{~cm}^{-3}$. In that experiment, the nucleation rate was estimated to be $20 \mathrm{~cm}^{-3} \mathrm{~s}^{-1}$. Multiplying by the volume of the chamber (198 L) gives a 
total particle generation rate of $4 \times 10^{6} \mathrm{~s}^{-1}$. For comparison, in a study in a lab room where limonene was released as a result of peeling oranges in the presence of $\sim 20 \mathrm{ppb}$ ozone, the calculated particle nucleation rate throughout the room was considerably smaller, $\sim 10^{5} \mathrm{~s}^{-1}$ (Vartiainen et al., 2006).

Similarly, a balance on particle mass concentration, $P_{M}$, considers formation from condensation of reaction products, balanced by removal by means of ventilation:

$$
\frac{d P_{M}}{d t}=R_{C}-\lambda P_{M} \quad R_{C}=\lambda P_{M, S S}
$$

Again, at steady state, the production rate of condensed material, $R_{C}$, must approximately equal the chamber air-exchange rate, $\lambda$, times the total measured steady-state particle mass concentration, $P_{M, S S}$. Supply air is again not included as a source, since the mass concentration of particles in the supply air is negligible compared with the concentration when ozone and terpenes are simultaneously present in the chamber. For example, in experiment POC-MH, the supply air contained $0.005 \mu \mathrm{g} \mathrm{m}^{-3}$ and the stage 4 concentration in the chamber was $76 \mu \mathrm{g} \mathrm{m}^{-3} . R_{C}$ represents only a fraction of the total production rate of reaction products, since some of the reaction products may persist in the gas phase or deposit on chamber surfaces. For the POC-MH experiment, the mass production rate of the particle-phase condensed material was $3.8 \mu \mathrm{g} \mathrm{m}^{-3} \mathrm{~min}^{-1}$. The steady-state particle nucleation and mass production rates calculated in this way for all experiments using the steady-state number and mass concentrations from Table 2-3 and the AER from Table 2-1 were in the range $1-23 \mathrm{~cm}^{-3} \mathrm{~s}^{-1}$ and $1-13 \mu \mathrm{g} \mathrm{m}^{-3} \mathrm{~min}^{-1}$, respectively, as reported in Table 2-4. 


\subsubsection{Modeling total particle mass formation}

Can a simple mechanistic model be used to describe the rate of increase in particle mass concentration during the initial parts of these experiments? The OOD experiments were used for this exercise because OOD only contains one reactive compound, $d$ limonene. I used a numerical approximation to solve coupled differential equations for the species considered: ozone $\left[\mathrm{O}_{3}\right]$, limonene $[L]$, and secondary particle mass $[\mathrm{SOA}]$ (equations 2-6-2-8).

$$
\begin{aligned}
& \frac{d\left[O_{3}\right]}{d t}=\lambda\left[O_{3}\right]_{\text {supply }}-\lambda\left[O_{3}\right]-k_{O 3}\left[O_{3}\right][L] \\
& \frac{d[L]}{d t}=\lambda[L]_{\text {supply }}-\lambda[L]-k_{O 3}\left[O_{3}\right][L] \\
& \frac{d[S O A]}{d t}=\lambda[S O A]_{\text {supply }}-\lambda[S O A]+Y k_{O 3}\left[O_{3}\right][L]
\end{aligned}
$$

Units of ppb were used for ozone and limonene concentrations in equations 2-6 and 2-7, but the limonene concentration was converted to units of $\mu \mathrm{g} \mathrm{m}^{-3}$ for equation 2-8. The SOA concentration was also expressed in units of $\mu \mathrm{g} \mathrm{m}^{-3}$. The reaction rate for ozone and limonene, $k_{O 3}$, was set to the experimentally determined value of $5.16 \times 10^{-6} \mathrm{ppb}^{-1} \mathrm{~s}^{-1}$ (Hakola et al., 1994). The yield, $Y$, was set to the value determined in the present study (see Figure 2-5b).

In the two cases in which the AER was relatively high (OOD-HH and OOD-MH), this model captured the overall shape of the increase in total particle mass concentration after the initial nucleation burst, but slightly underestimated particle mass. However, in

the low AER case (OOD-HL), the model substantially underpredicted both the total mass of SOA formed and the rate of formation (Figure 2-7). The model may underpredict mass 
formation because it considers only reactions of ozone and limonene, whereas the experimentally-determined yield is an overall value that incorporates all factors contributing to SOA mass. Other factors include the formation and condensation of second-generation oxidation products (see Section 2.5.1.) and uptake of water by the hygroscopic SOA (see Section 2.5.2.).

\subsection{Conclusions}

In this study, data from a series of chamber experiments were used to characterize the dynamics of particle formation and growth from ozone reactions with terpenecontaining vapors originating from consumer products under indoor-relevant conditions. Particles were measured with an SMPS (10-400 nm) and an OPC (0.1-1.1 $\mu \mathrm{m})$. The particles formed were in the ultrafine and accumulation modes $(<1.1 \mu \mathrm{m})$, and the mass concentrations of particles $\left(\mathrm{PM}_{1.1}\right)$ ranged from the tens to hundreds of $\mu \mathrm{g} \mathrm{m}^{-3}$. For comparison, yearly maximum 24-hour ambient $\mathrm{PM}_{2.5}$ concentrations are in the same range (EPA, 2008). Hence, relative to health-based standards, this evidence suggests that indoor reactions between ozone and terpenes can be a significant source of particles, warranting further study of potential exposure-related effects.

In each of the 16 experiments, a burst of particle formation by nucleation occurred immediately after ozone addition. This burst was followed by a period characterized predominantly by condensational growth of the nucleated particles. The system then evolved through a third stage to a fourth during which particle nucleation and growth persisted under steady state or cyclic conditions for the remainder of the experiment. At higher air-exchange rates, the particle surface area was reduced rapidly as particles were 
swept out of the chamber, and nucleation seemed to occur continuously. In the lower AER experiments, an oscillating dynamic balance between formation and growth was exhibited. Mass and yield of SOA were observed to increase with increasing ozone level for the range of ozone levels likely to be encountered under normal indoor conditions. More SOA was formed per unit precursor consumed when the air-exchange rate was lowered; the additional SOA may be attributable to second-generation oxidation processes.

In any real environment there would likely be much greater spatial heterogeneity than in these small-chamber experiments. SOA formation and growth has been measured in realistic settings and has exhibited similar characteristics (size distribution and growth dynamics) to the SOA production measured in these more controlled experiments.

Studies such as the one presented here help to elucidate the distribution and evolution of particles that people are likely exposed to when vapors from terpene-containing cleaning products or air fresheners are simultaneously present with ozone indoors. 
Table 2-1: Conditions for experiments where ozone and terpene-containing consumer product vapors were mixed in a continuously ventilated $198 \mathrm{~L}$ Teflon-lined chamber. ${ }^{\mathrm{a}}$

\begin{tabular}{cccccc}
\hline Exp. ID & $\begin{array}{c}\text { Supply } \\
\text { ozone } \\
\text { level } \\
(\mathrm{ppb})\end{array}$ & $\begin{array}{c}\text { Residual } \\
\text { ozone level } \\
(\mathrm{ppb})\end{array}$ & $\begin{array}{c}\text { Air- } \\
\text { exchange } \\
\text { rate }\left(\mathrm{h}^{-1}\right)\end{array}$ & $\begin{array}{c}\text { Supply } \\
\text { terpene level } \\
(\mathrm{ppb})^{\mathrm{b}}\end{array}$ & $\begin{array}{c}\text { Residual } \\
\text { terpene } \\
\text { level } \\
(\mathrm{ppb})\end{array}$ \\
\hline \hline OOD-HH & 137 & 21 & 3.0 & 643 & 518 \\
OOD-HL & 136 & 7 & 1.0 & 738 & 588 \\
OOD-MH & 61 & 11 & 3.1 & 586 & 528 \\
\hline AFR-HH & 126 & 18 & 3.0 & 623 & 492 \\
AFR-HL & 127 & 4 & 1.0 & 859 & 658 \\
AFR-MH & 63 & 7 & 3.0 & 596 & 506 \\
\hline POC-VH & 253 & 25 & 3.0 & 716 & 439 \\
POC-HH1 & 131 & 13 & 3.0 & 771 & 560 \\
POC-HH2 & 121 & 12 & 3.0 & 692 & 531 \\
POC-HL & 130 & 8 & 1.0 & 735 & 599 \\
POC-MH & 65 & 2 & 3.0 & 734 & 673 \\
POC-LH & 29 & 0 & 3.1 & 566 & 540 \\
POC-NOx & 139 & 8 & 3.0 & 817 & 615 \\
POC-Rev & 120 & - & 3.0 & - & - \\
POC-Seed & 125 & - & 3.0 & - & - \\
POC-Dry & 63 & - & 3.0 & 558 & 512 \\
\hline
\end{tabular}

${ }^{a}$ For experimental identification, the pre-dash letters denote the cleaning product and the post-dash letters denote some key aspect of experimental conditions, as follows: "HH" indicates high ozone level ( $\sim 130 \mathrm{ppb}$ at the inlet) and high air-exchange rate (AER $=3$ $\left.\mathrm{h}^{-1}\right)$; "HL" indicates high ozone level and low AER $\left(1 \mathrm{~h}^{-1}\right)$; and "MH" indicates moderate ozone level ( $\sim 60 \mathrm{ppb}$ at the inlet) and high AER. Extra experiments were conducted with POC: "VH" denotes very high supply ozone (253 ppb at the inlet) and high AER; "HH1" and "HH2" are replicate experiments under HH conditions; "LH" indicates low ozone level (29 ppb at the inlet) and high AER. See text for a description of the remaining four POC experiments.

${ }^{b}$ Terpene composition of each product for each experiment is given in Tables $2 \mathrm{~B}-1$ (OOD), 2B-2 (AFR), and 2B-3 (POC). 
Table 2-2: Modeled three-mode lognormal SOA distribution parameters.

\begin{tabular}{|c|c|c|c|c|c|c|c|c|c|}
\hline \multirow[b]{2}{*}{ Exp. ID } & \multicolumn{3}{|c|}{ Mode 1} & \multicolumn{3}{|c|}{ Mode 2} & \multicolumn{3}{|c|}{ Mode 3} \\
\hline & $\begin{array}{c}\mathrm{N} \\
\left(\mathrm{cm}^{-3}\right) \\
\end{array}$ & $\begin{array}{l}\text { GMD } \\
(\mathrm{nm})\end{array}$ & GSD & $\begin{array}{c}\mathrm{N} \\
\left(\mathrm{cm}^{-3}\right) \\
\end{array}$ & $\begin{array}{l}\text { GMD } \\
(\mathrm{nm})\end{array}$ & GSD & $\begin{array}{c}\mathrm{N} \\
\left(\mathrm{cm}^{-3}\right) \\
\end{array}$ & $\begin{array}{l}\text { GMD } \\
(\mathrm{nm})\end{array}$ & GSD \\
\hline OOD-HH & 2300 & 48 & 1.6 & 7000 & 135 & 1.6 & 8700 & 314 & 1.4 \\
\hline OOD-HL* & 1500 & 40 & 1.5 & 5000 & 105 & 1.5 & 6000 & 310 & 1.4 \\
\hline OOD-HL* & 1500 & 70 & 1.6 & 2500 & 140 & 1.5 & 7500 & 330 & 1.4 \\
\hline OOD-MH & 2200 & 45 & 1.6 & 5700 & 123 & 1.6 & 4700 & 265 & 1.4 \\
\hline AFR-HH & 2200 & 45 & 1.6 & 3200 & 125 & 1.5 & 2700 & 280 & 1.35 \\
\hline AFR-HL & 1300 & 55 & 1.7 & 1700 & 140 & 1.5 & 3300 & 300 & 1.4 \\
\hline AFR-MH & 1600 & 35 & 1.55 & 2900 & 100 & 1.6 & 1800 & 222 & 1.4 \\
\hline POC-VH & 3000 & 45 & 1.6 & 5000 & 130 & 1.6 & 7500 & 315 & 1.4 \\
\hline POC-HH1 & 2500 & 48 & 1.6 & 4500 & 140 & 1.6 & 4700 & 316 & 1.4 \\
\hline POC-HH2 & 3000 & 60 & 1.7 & 3080 & 138 & 1.5 & 6000 & 295 & 1.4 \\
\hline POC-HL* & 1500 & 80 & 1.7 & 5000 & 140 & 1.4 & 6000 & 243 & 1.3 \\
\hline POC-HL* & 3000 & 70 & 1.7 & 1400 & 211 & 1.35 & 4900 & 305 & 1.3 \\
\hline POC-MH & 1300 & 25 & 1.5 & 13000 & 75 & 1.7 & 7000 & 223 & 1.4 \\
\hline POC-LH & 4000 & 42 & 1.65 & 3800 & 110 & 1.56 & 1400 & 182 & 1.45 \\
\hline POC-NOx & 2900 & 37 & 1.55 & 5500 & 115 & 1.6 & 5000 & 300 & 1.4 \\
\hline POC-Rev & 2000 & 40 & 1.6 & 4500 & 120 & 1.6 & 6000 & 295 & 1.4 \\
\hline POC-Seed & 9000 & 55 & 1.75 & 7000 & 105 & 1.5 & 15000 & 225 & 1.5 \\
\hline POC-Dry & 1300 & 60 & 1.8 & 2000 & 130 & 1.5 & 3000 & 275 & 1.4 \\
\hline
\end{tabular}

* Indicates experiments where the size distribution varied significantly in stage 4 (steadystate) and two sets of distribution parameters are given, corresponding to minimum and maximum values. 
Table 2-3: Measured and modeled SOA number and mass concentrations.

\begin{tabular}{|c|c|c|c|c|c|c|c|c|}
\hline \multirow{3}{*}{ Exp. ID } & \multicolumn{6}{|c|}{ Measured } & \multirow{2}{*}{\multicolumn{2}{|c|}{$\begin{array}{c}\text { Modeled } \\
\text { Steady-state } \\
\text { mass conc. } \\
\left(\mu \mathrm{g} \mathrm{m}^{-3}\right)\end{array}$}} \\
\hline & \multirow{2}{*}{$\begin{array}{l}\text { Peak } \\
\text { number } \\
\text { conc. } \\
\left(10^{5}\right. \\
\left.\mathrm{cm}^{-3}\right)\end{array}$} & \multicolumn{2}{|c|}{$\begin{array}{c}\text { Peak mass } \\
\text { conc. } \\
\left(\mu \mathrm{g} \mathrm{m}^{-3}\right)\end{array}$} & \multirow{2}{*}{$\begin{array}{l}\text { Steady- } \\
\text { state } \\
\text { number } \\
\text { conc. } \\
\left(10^{5} \mathrm{~cm}^{-3}\right)\end{array}$} & \multicolumn{2}{|c|}{$\begin{array}{l}\text { Steady-state } \\
\text { mass conc. } \\
\left(\mu \mathrm{g} \mathrm{m}^{-3}\right)\end{array}$} & & \\
\hline & & $\mathrm{PM}_{0.4}$ & $\mathrm{PM}_{1.1}$ & & $\mathrm{PM}_{0.4}$ & $\mathrm{PM}_{1.1}$ & $\mathrm{PM}_{0.4}$ & $\mathrm{PM}_{1.1}$ \\
\hline OOD-HH & 1.4 & 229 & - & 0.18 & 105 & - & 126 & 259 \\
\hline OOD-HL* & 0.8 & 192 & - & 0.13 & 71 & - & 87 & 162 \\
\hline OOD-HL* & & & & 0.10 & 87 & - & 99 & 243 \\
\hline OOD-MH & 0.6 & 75 & - & 0.13 & 53 & - & 63 & 92 \\
\hline AFR-HH & 0.4 & 51 & 93 & 0.08 & 33 & 80 & 39 & 54 \\
\hline AFR-HL & 0.9 & 90 & 146 & 0.05 & 37 & 110 & 44 & 83 \\
\hline AFR-MH & 0.2 & 21 & 35 & 0.07 & 15 & 29 & 18 & 21 \\
\hline POC-VH & 3.6 & 306 & 435 & 0.16 & 107 & 215 & 104 & 220 \\
\hline POC-HH1 & 2.3 & 174 & 273 & 0.12 & 67 & 115 & 72 & 147 \\
\hline POC-HH2 & 2.4 & 162 & - & 0.11 & 66 & - & 79 & 144 \\
\hline POC-HL* & 1.6 & 102 & - & 0.14 & 58 & - & 69 & 75 \\
\hline POC-HL* & & & & 0.10 & 75 & - & 78 & 111 \\
\hline POC-MH & 1.2 & 57 & - & 0.24 & 50 & - & 64 & 76 \\
\hline POC-LH & 0.5 & 12 & 35 & 0.10 & 11 & 19 & 14 & 15 \\
\hline POC-NOx & 1.8 & 146 & 215 & 0.14 & 64 & 185 & 70 & 130 \\
\hline POC-Rev & 1.9 & 149 & 220 & 0.12 & 67 & 175 & 80 & 145 \\
\hline POC-Seed & 3.2 & 141 & 225 & 0.28 & 100 & 212 & 130 & 200 \\
\hline POC-Dry & 0.9 & 62 & - & 0.06 & 31 & - & 38 & 60 \\
\hline
\end{tabular}

* Indicates experiments where the size distribution varied significantly in stage 4 (steadystate) and two sets of distribution parameters are given, corresponding to minimum and maximum values. 
Table 2-4: SOA number and mass formation rates.

\begin{tabular}{ccc}
\hline Exp. ID & $\begin{array}{c}\mathrm{R}_{\mathrm{N}, \mathrm{Ss}} \\
\left(\mathrm{cm}^{-3} \mathrm{~s}^{-1}\right)\end{array}$ & $\begin{array}{c}\mathrm{R}_{\mathrm{M}, \mathrm{Ss}} \\
\left(\mu \mathrm{g} \mathrm{m}^{-3} \mathrm{~min}^{-1}\right)\end{array}$ \\
\hline \hline OOD-HH & 15 & 13 \\
OOD-HL* & 4 & 3 \\
OOD-HL* & 3 & 4 \\
OOD-MH & 11 & 5 \\
\hline AFR-HH & 7 & 3 \\
AFR-HL & 1 & 1 \\
AFR-MH & 6 & 1 \\
\hline POC-VH & 13 & 11 \\
POC-HH1 & 10 & 7 \\
POC-HH2 & 9 & 7 \\
POC-HL* & 4 & 1 \\
POC-HL* & 3 & 2 \\
POC-MH & 20 & 4 \\
POC-LH & 8 & 1 \\
POC-NOx & 12 & 7 \\
POC-Rev & 10 & 7 \\
POC-Seed & 23 & 10 \\
POC-Dry & 5 & 3 \\
\hline
\end{tabular}

* Indicates experiments where the size distribution varied significantly in stage 4 (steadystate) and two sets of distribution parameters are given, corresponding to minimum and maximum values. 


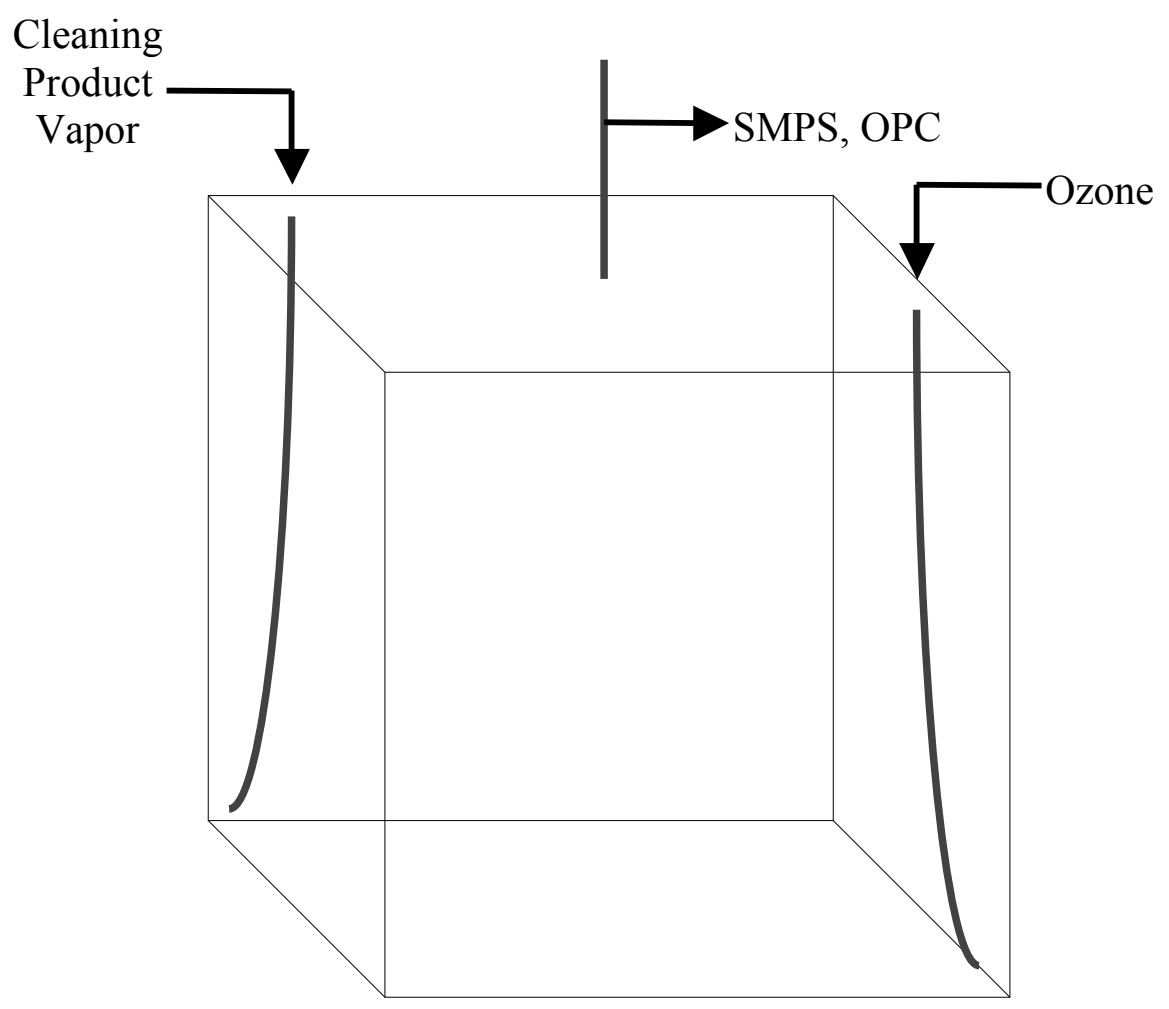

Figure 2-1: Diagram of experimental apparatus for the measurement of byproducts from gas-phase reactions of ozone and terpene-containing consumer products. A steady level of consumer product vapor was introduced into the continuously ventilated $198 \mathrm{~L}$ Teflonlined chamber, to which a steady level of ozone was added. Cleaning product vapor and ozone were introduced through Teflon tubing in opposite bottom corners of the chamber. Integrated gas-phase measurements and continuous particle measurements were made at the top middle of the chamber. 
(a) POC-HH

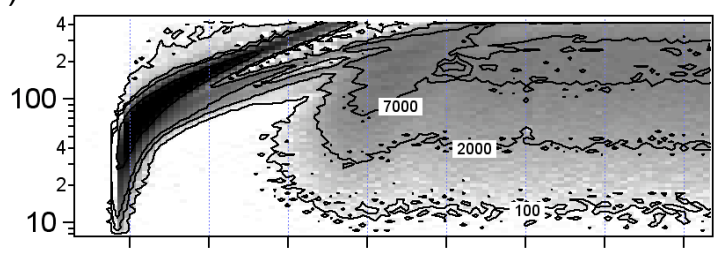

(b) POC-MH

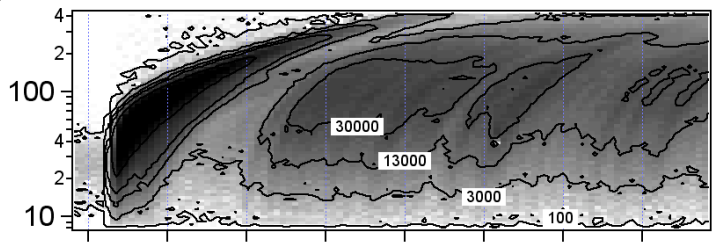

(c) POC-Dry

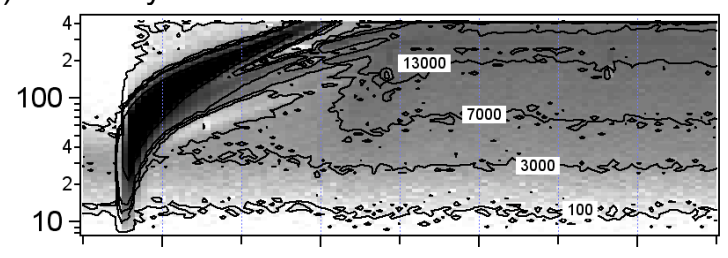

(d) POC-Rev

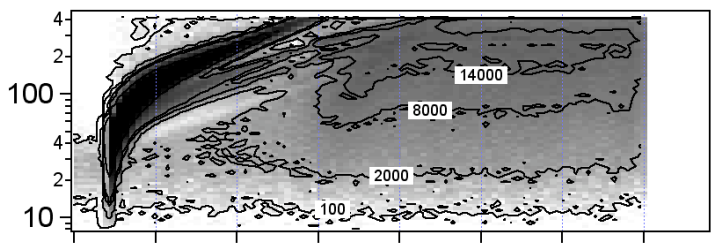

(e) POC-Seed

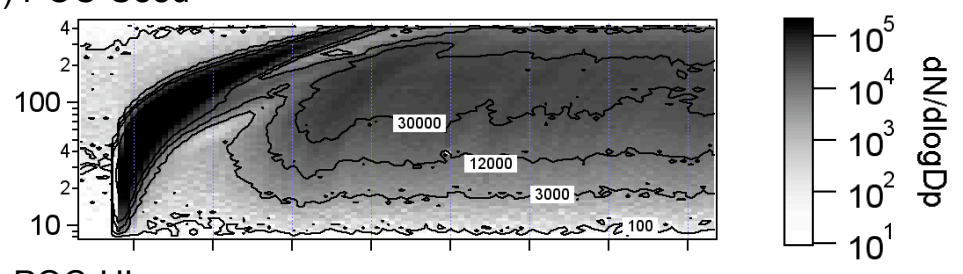

(f) POC-HL

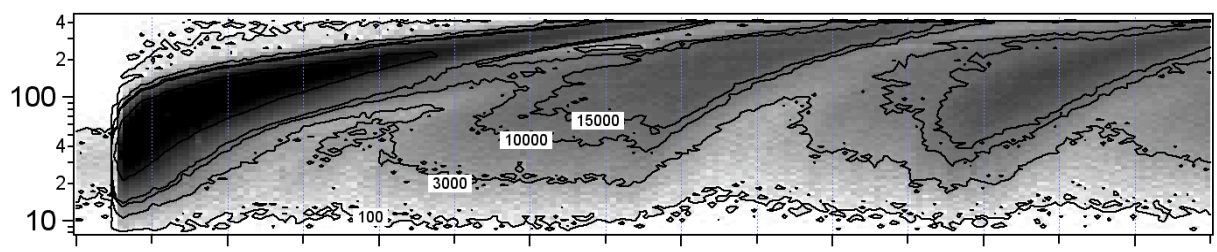

Time (half-hour increments are marked)

Figure 2-2: Particle size-distribution evolution as measured with an SMPS for six experiments in which vapor from a pine-oil cleaner (POC) was combined with ozone under different conditions. The y-axis indicates particle diameter, $\mathrm{D}_{\mathrm{p}}(\mathrm{nm})$, the $\mathrm{x}$-axis represents time (with tick marks indicating 30-min increments), and the shading and numerical isopleth labels indicate the count-based particle size distribution, $d N / d\left(\log D_{p}\right)$ $\left(\mathrm{cm}^{-3}\right)$. Nucleation begins with the onset of ozone supply to the chamber that already contains POC vapors. 


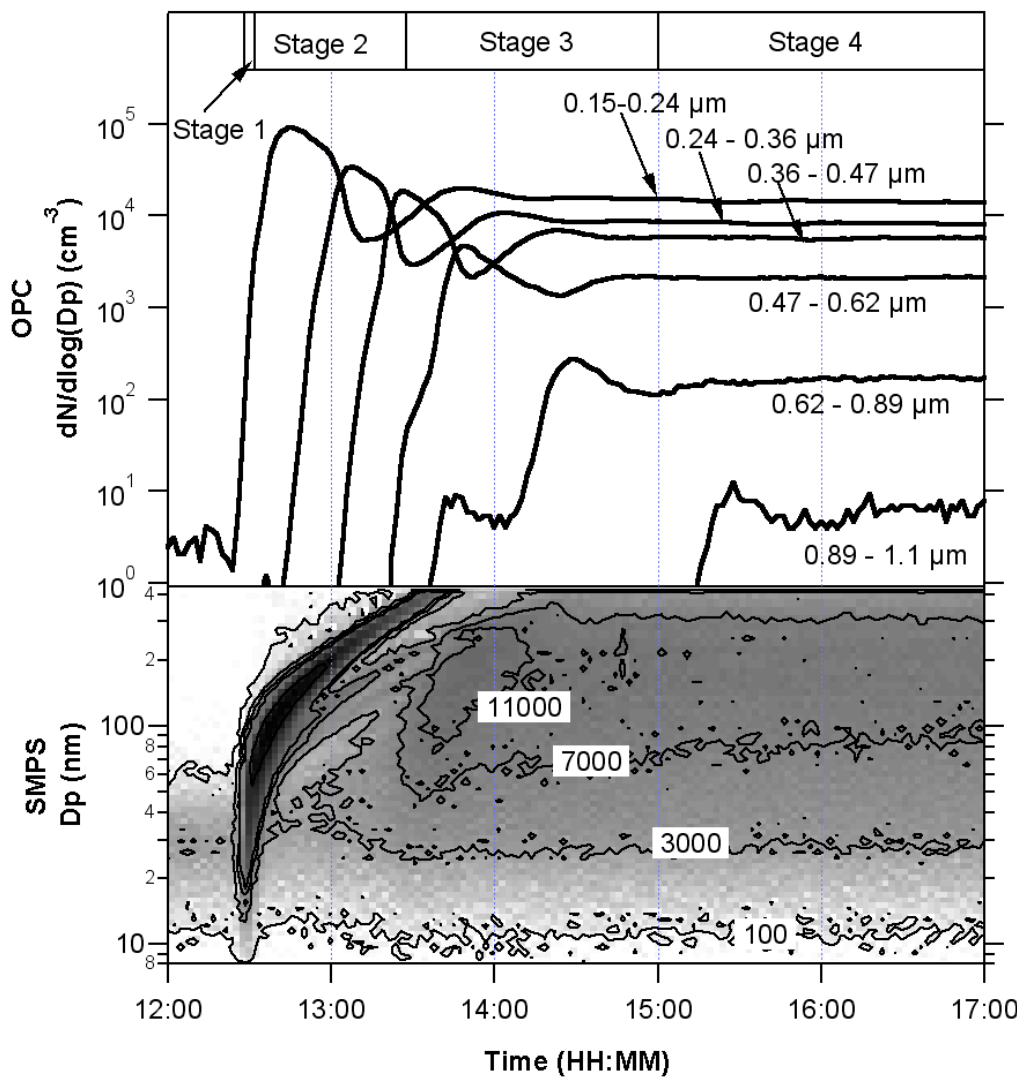

Figure 2-3: Characteristic stages of particle formation and growth in chamber experiments, illustrated for experiment AFR-HH. Stage 1 is characterized by a sudden nucleation burst when ozone is added to the chamber containing a steady level of product vapor. Stage 2 is characterized by the growth of particles from the initial nucleation burst. In stage 3 nucleation resumes but particles from the initial nucleation event are still present. In stage 4 a steady or oscillating particle concentration is achieved. The scale for $\mathrm{dN} / \mathrm{d}\left(\log \mathrm{D}_{\mathrm{p}}\right)$ in the lower frame is the same as in Figure 2-2. 


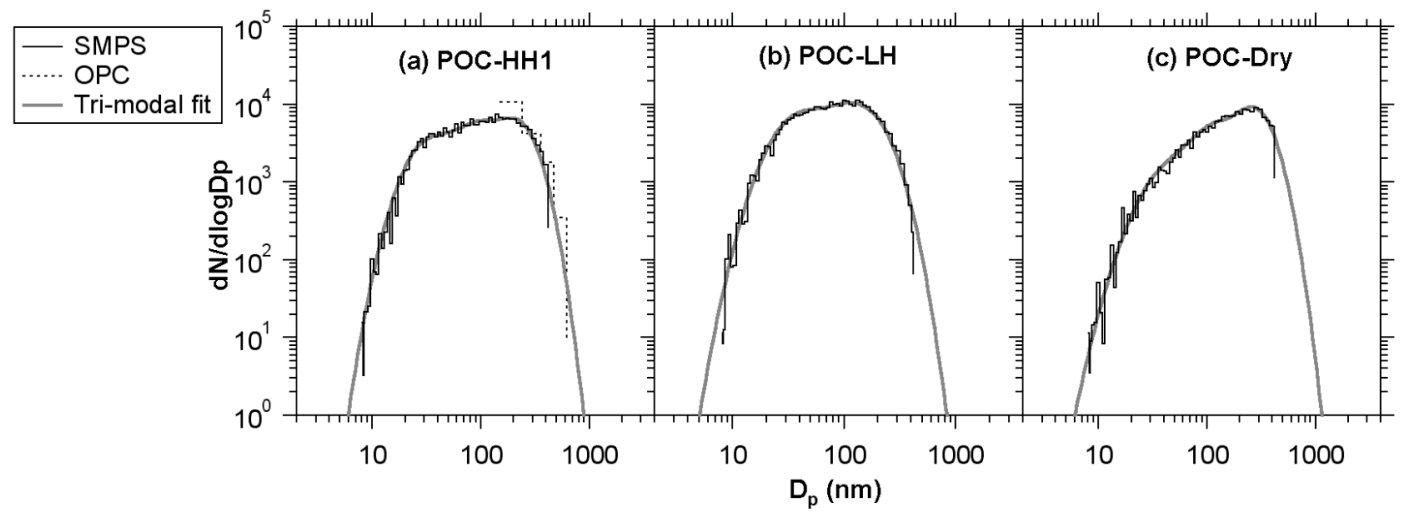

Figure 2-4: Size distributions measured with an SMPS and an OPC and fit using the sum of three lognormal distributions. The $\mathrm{dN} / \mathrm{d}\left(\log \mathrm{D}_{\mathrm{p}}\right)$ scale has units of $\mathrm{cm}^{-3}$. 

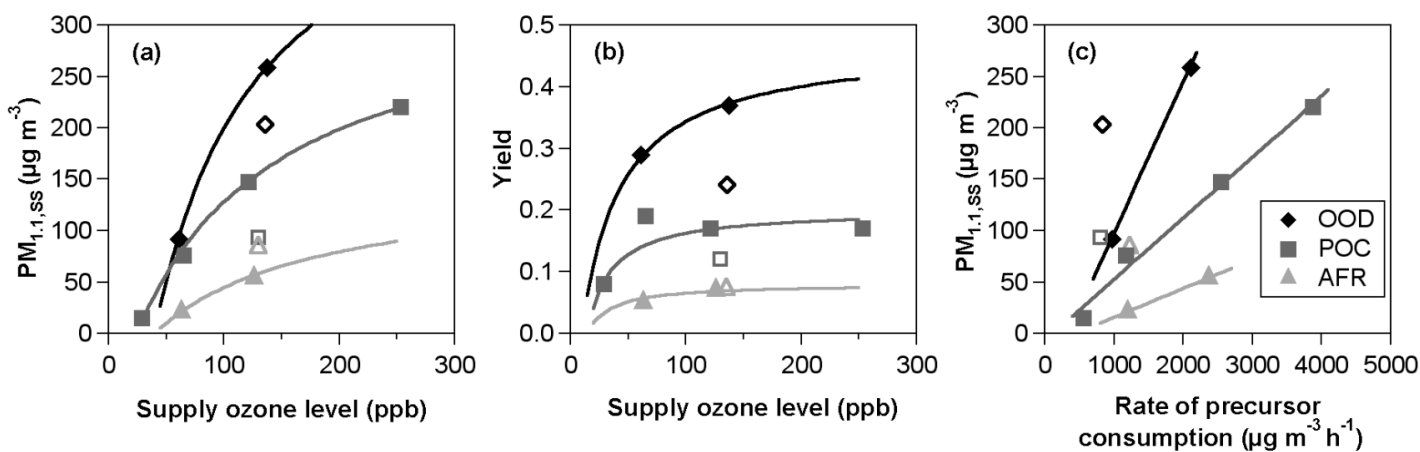

Figure 2-5: Effect of ozone level and air-exchange rate on steady-state particle mass concentration $\left(\mathrm{PM}_{1.1, \mathrm{SS}}\right)$ and yield. Solid symbols correspond to an air-exchange rate (AER) of $3 \mathrm{~h}^{-1}$ and hollow symbols indicate AER $=1 \mathrm{~h}^{-1}$. Lines are drawn to guide the eye using a Langmuir fit in (a) and (b) and linear fit in (c) to the high AER data. Mass and yield increase with increasing ozone supply level and the SOA-forming potential of the terpene constituents (OOD $>$ POC $>$ AFR) for a constant air exchange rate $\left(3 \mathrm{~h}^{-1}\right)$. At lower air-exchange rates, more SOA mass is formed per rate of precursor consumption. 


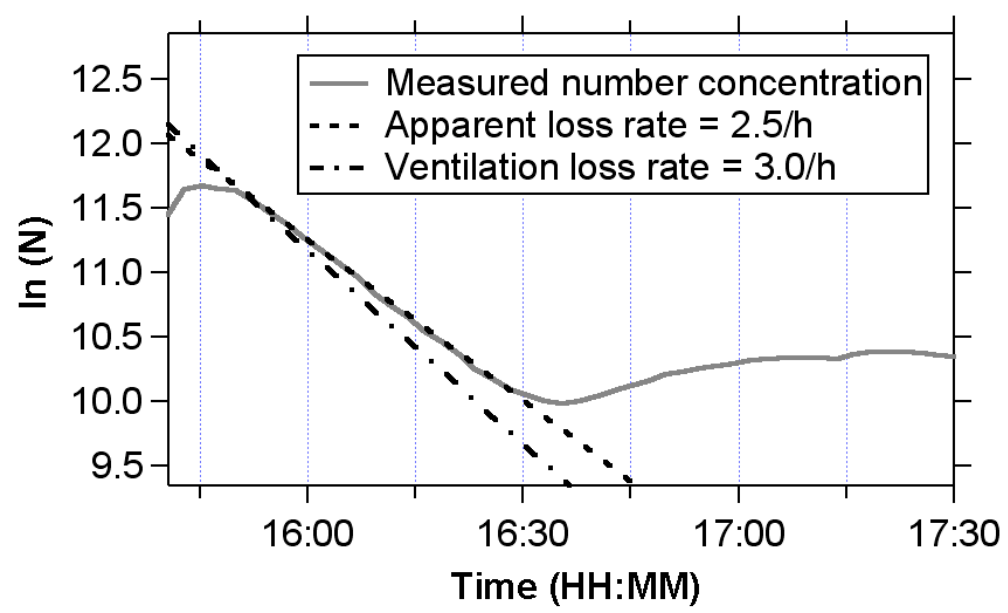

Figure 2-6: Comparison of measured particle number decay with that expected from removal by ventilation alone, for experiment POC-MH during stage 2 . The solid line shows the total SMPS particle number concentration, $N\left(\mathrm{~cm}^{-3}\right)$, and lines are superimposed for apparent loss rate (dash) and loss expected from ventilation alone (dash-dot). 


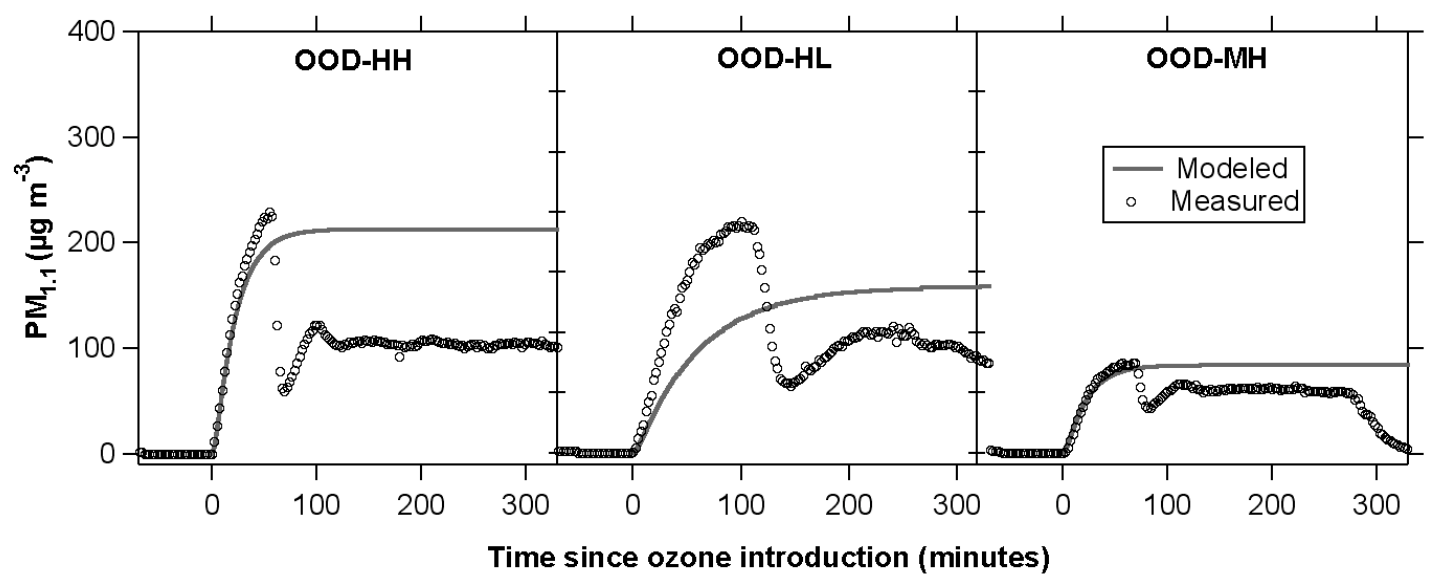

Figure 2-7: Modeled and measured particle mass concentrations vs. time for three experiments using the orange-oil degreaser (OOD), which contained d-limonene. The sudden decrease in particle mass at time $\sim 100 \mathrm{~min}$ in each experiment results from growth of the largest particles out of the size range of the SMPS. 


\section{A. Appendix: Codes for particle distribution analysis}

The following code was written in the Igor programming language. The main function, "MatchOPCandSMPS," and its sub-functions comprise a routine that determines the refractive index that produces optimal alignment between data collected with an SMPS and an OPC (see Section 2.3.2.). The function "CalcNSV" requires input of lognormal distribution parameters for three modes, and calculates the total particle number, surface area, and volume of a distribution (from $1 \mathrm{~nm}$ to $10 \mu \mathrm{m}$ ), and the number, area, and volume that would be measured by the SMPS (i.e. for particles with diameter less than $400 \mathrm{~nm}$ ). This program was used to calculate "modeled" steady-state particle mass (Table 2-3) given the three-mode, lognormal parameters determined from a manual fit (Table 2-2). The function "CalcCondensationCharTime" was used to calculate the characteristic time for condensation of vapor onto particles using the Fuchs and Sutugin approach (equation 2-2). Figure 2A-1 illustrates the output of "CalcCondensationCharTime". 
Function MatchOPCandSMPS(DDMonYY_Time,MonDD)

//This function was created to match SMPS and OPC data by changing the OPC bin bounds according to the Hand polynomials.

//The output of this function is best fit refractive index for the SMPS data at each time point string DDMonYY_Time, MonDD

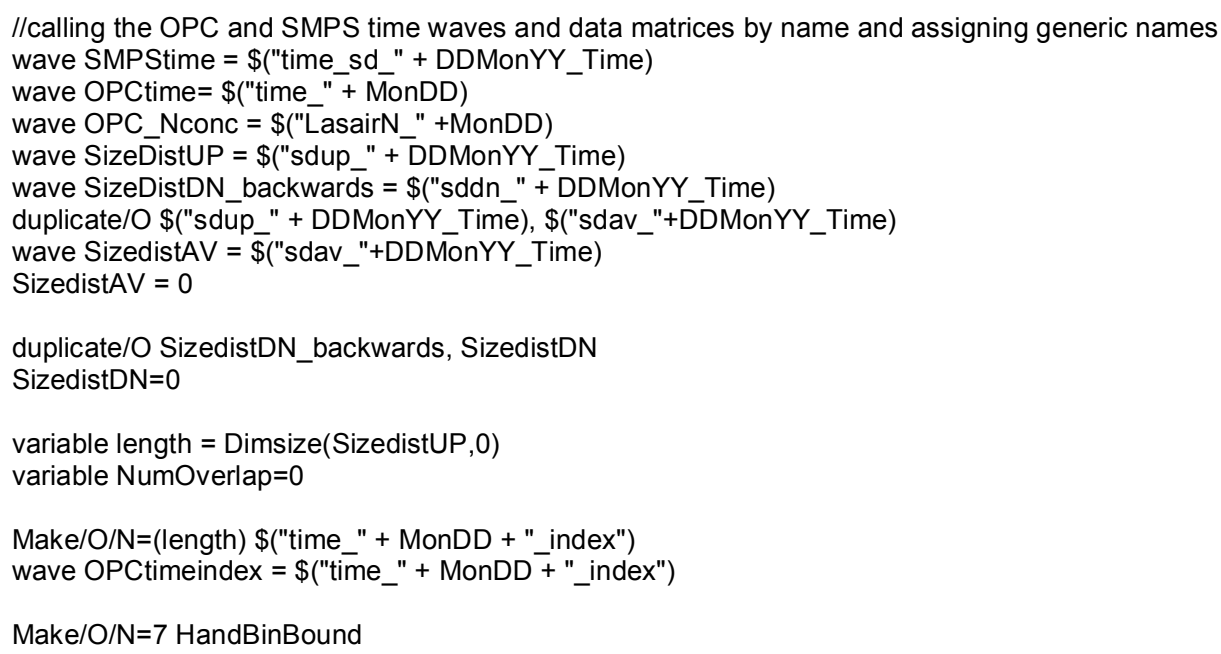


MatchDistAV[][k] =

Binfraction[k] SizedistAV[p][LowerBB]*log(dp_smps[LowerBB+1]/dp_smps[LowerBB]) + (1+

Binfraction[k+1])*SizedistAV[p][UpperBB] ${ }^{*} \log \left(\mathrm{dp} \_s m p s[U p p e r B B+1] / d p \_s m p s[U p p e r B B]\right)$

variable $n=0$

do

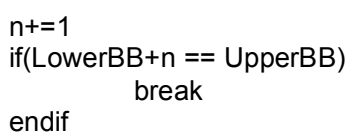




\author{
//Endfor \\ //Choose the refractive that minimizes least squares for each case \\ End \\ Endfor
}

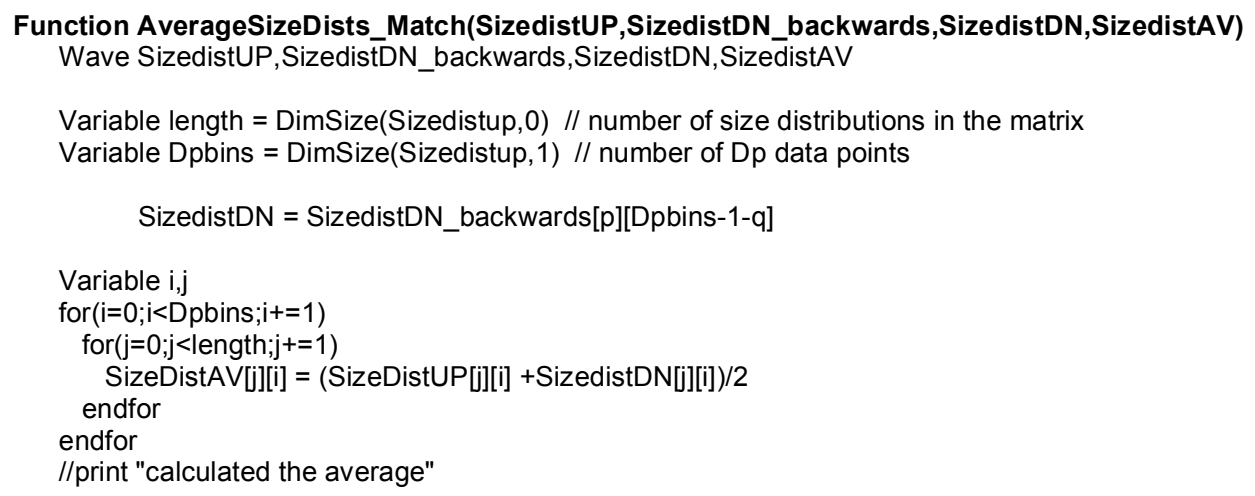

End

Function MakeOPCtimelndex(SMPStime, OPCtime, OPCtimeindex) wave SMPStime, OPCtime, OPCtimeindex

variable smps_length $=$ numpnts $($ smpstime $)$

variable opc_length $=$ numpnts $($ opctime $)$

Variable $k=0, i, j=0$, diff1, diff2, breakflag $=0$

I/This do loop sets the first value(s) of the OPC index to 0 (placeholders for when the OPC data starts later than the SMPS data)

do

OPCtimeindex $[\mathrm{k}]=0$

$\mathrm{k}+=1$

while (OPCtime[0] > SMPStime[k])

//this for loop cycles through each SMPS time point determining which OPC time point is closest

for(i=k; i< (smps_length); i+=1)

diff1 $=0$; diff2=0

//this do loop picks out the OPC time right before the SMPS time

do

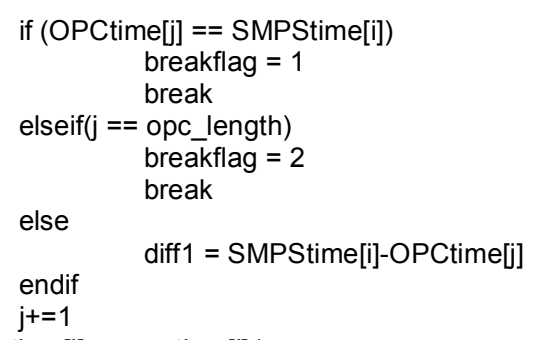

while(opctime[j]< smpstime[i])

diff2 = OPCtime[j]- SMPStime[i]

//this if statement compares which OPC time on either side of the SMPS time point is closest

if $($ breakflag $==1$ )

OPCtimeindex $[\mathrm{i}]=\mathrm{j}$; breakflag $=0$

elseif (breakflag $==2$ )

OPCtimeindex $[i]=$ opc length

elseif ( diff1> diff2 )

OPCtimeindex[i] $=\mathrm{j}$

else

$$
\text { OPCtimeindex }[\mathrm{i}]=\mathrm{j}-1
$$




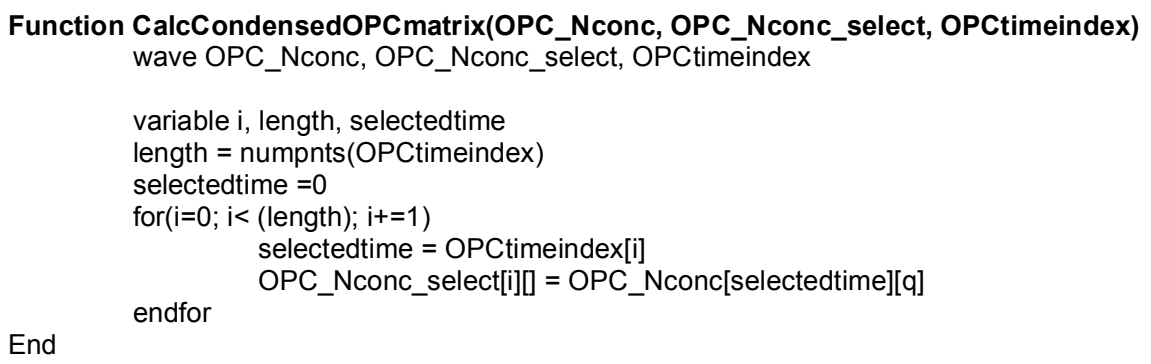

Function CalculateHandBinBounds(m)

//This function uses polynomials developed by Jenny Hand (disseration) to calculate the OPC bin bounds for a particular refractive index.

Variable $\mathrm{m}$

Wave HandBinBound

$/ / \mathrm{m}$ is the refractive index of the compound, e.g.1.46 for oleic acid

Wave coeffA $/ /=\{19.637,9.7348,7.1322,-2.1338,2.8073,9.6226,1.5827\}$

Wave coeffB $/ /=\{-57.25,-28.37,-20.437,7.6673,-7.0444,-27.662,-4.1139\}$

Wave coeffC $/ /=\{42.393,21.502,15.468,-5.7949,5.1074,20.661,3.5417\}$

Wave DefaultOPCbound $/ /=\{0.1,0.2,0.3,0.4,0.5,0.7,1.0\}$

//do the math on the bin sizes

Variable i

For $(i=0 ; i<7 ; i+=1)$

endfor

HandBinBound $[i]=$ DefaultOPCbound $[i] /\left(\operatorname{coeffA}[i]^{*} m^{\wedge} 2+\operatorname{coeffB}[i]^{*} m+\operatorname{coeffC}[i]\right)$

//print HandBinBound, " in micrometers for refractive index: ", m end

\section{Function FindOverlappingBinFractions(NumOverlapBinBounds)}

Variable \&NumOverlapBinBounds

Wave HandBinBound

HandBinBound $=$ HandBinBound ${ }^{*} 1000 / /$ converts from um to $\mathrm{nm}$

Variable dp_index, j

Wave dp_smps //generic smps bin bounds (65 points)

If (HandBinBound[3] <= dp_smps[63])

Else

NumOverlapBinBounds $=4$

Endif

NumOverlapBinBounds $=3$

Make/O/N=(NumOverlapBinBounds) SMPSbinLocn, BinFraction

For(j=0;j<(NumOverlapBinBounds); $j+=1$ )

FindSMPSbinLocation(HandBinBound[j],dp_index)

SMPSbinLocn[j] $=\mathrm{dp}$ index

dp_smps[dp_index])

Binfraction[j] $=\left(\mathrm{dp} \_\right.$smps $\left[\mathrm{dp} \_\right.$index+1] - HandBinBound[j] $) /\left(\mathrm{dp} \_s m p s\left[\mathrm{dp} \_\right.\right.$index +1$]-$

Endfor 
//print SMPSbinLocn

//print Binfraction

End

Function FindSMPSbinLocation(Dp_interest,lowerSMPSbinLocn)

I/This function takes a particle diameter of interest and tells you what SMPS bin is just before that value.

//dp_smps (nm) is the wave with the 65 SMPS bin bounds

//Dp_interest (nm) is a value of an OPC bin bound for example

//lowerSMPSbinLocn is the output, it tells the row of the lower SMPS bin

variable $\mathrm{Dp}$ _interest, \&lowerSMPSbinLocn

variable $\mathrm{i}=0$

file)

wave dp_smps //this is the ascending smps bin bounds in nanometers (same as sdup_or sddn_for any data

do

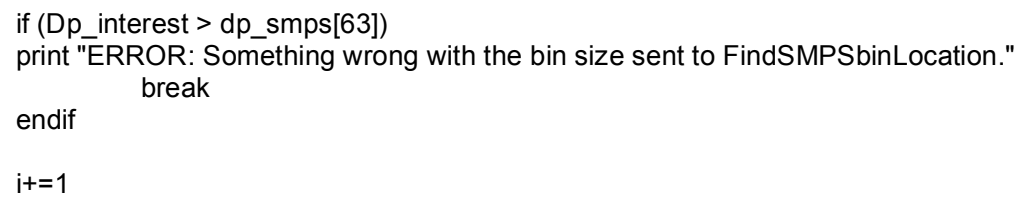

End 
Function CalcNSV(MonDD, pointnum)

//This function calculates the Number Surface and Volume of a particle distribution

$/ /$ in the range $1 \mathrm{~nm}$ to 10 um given the parameters for three modes.

//The N, S, and V that would be measured by the SMPS is also given (10nm to $400 \mathrm{~nm}$ )

\section{string MonDD}

variable pointnum

variable interval $=0.05$

variable N_total, S_total, V_total, N_k, S_k, V_k

$\mathrm{N}$ total $=0 ; \mathrm{S}$ total $=0 ; \mathrm{V}$ total $=0 ; \mathrm{N} \mathrm{k}=0 ; \mathrm{S} \mathrm{k}=0 ; \mathrm{V} \mathrm{k}=0$

variable N_SMPS, S_SMPS, V_SMPS, $N \_\bar{j}, S j, \bar{V}_{j}$

N_SMPS $=0 ;$ S_SMPS $=0 ; V \_S M P S=0 ; N \_j=0 ; S j=0 ; V \_j=0$

variable $\mathrm{k}=0$

variable $\mathrm{j}=0$

variable $\mathrm{Dp} \_\mathrm{i}=0$

wave tri mode parameters

//the wave "tri_mode_parameters" is where the three-mode dist. parameters are stored in the following order

// \{N1, GMD1, GSD1, N2, GMD2, GSD2, N3, GMD3, GSD3\}

$\operatorname{For}(k=0 ; k<9 ; k+=3)$

Dp_i $=0.001 / /$ units $=$ um

do

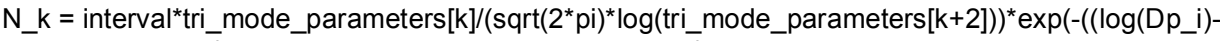

$\left.\left.\log (\text { tri_mode_parameters[k+1]/1000) })^{\wedge^{\wedge}} 2\right) /\left(2^{*}\left(\log \left(\operatorname{tri} \_ \text {mode_parameters }[k+2]\right)\right)^{\wedge} 2\right)\right)$

S_k $=$ N_k $k^{*} D p \_i^{\wedge} 2^{*} p i$

V_k $=N_{-} k^{*} D p_{-} i^{\wedge} 3^{*} p i / 6$

N_total=N_total $+\mathrm{N} \_\mathrm{k}$

S_total $=$ S_total $+S \_k$

V_total $=V_{-}$total $+V_{-}$k

Dp_i $=$ Dp_i*10^interval

Endfor

while $\left(\mathrm{Dp}_{-} \mathrm{i}<15\right)$

V_total

printf "N_total $=\% 8.0 \mathrm{fm}-3, \quad S \_t$ total $=\% 6.0 \mathrm{fmm} 2 \mathrm{~cm}-3, \quad \mathrm{~V} \_$total $=\% 4.0 \mathrm{~nm} 3 \mathrm{~cm}-3 \mid \mathrm{r}$ ", N_total, S_total,

For $(\mathrm{j}=0 ; j<9 ; j+=3)$

Dp_i $=0.010 / /$ units $=u m$

do

$\mathrm{N} \_\mathrm{j}=$ interval ${ }^{*}$ tri_mode_parameters[j]/(sqrt(2* $\left.2^{*}\right)^{\star} \log (\operatorname{tri} \text { mode_parameters[j+2])) })^{*} \exp \left(-\left(\left(\log \left(\mathrm{Dp} \_\mathrm{i}\right)-\right.\right.\right.$

$\log ($ tri_mode_parameters $\left.\left.[j+1] / 1000))^{\wedge} 2\right) /\left(2^{*}\left(\log \left(\operatorname{tri} \_m o d e \_p a r a m e t e r s[j+2]\right)\right)^{\wedge} 2\right)\right)$

$\mathrm{S} j=\mathrm{N} \mathrm{j}^{*} \mathrm{Dp} \mathrm{i}^{\wedge} 2^{*} \mathrm{pi}$

$V \_j=N j^{*} D p \_i^{\wedge} 3^{*} p i / 6$

$\mathrm{N} S M P S=\mathrm{N} S M P S+\mathrm{N}$

S_SMPS $=S \_S M P S+S+j$

V_SMPS $=$ V_SMPS $+\mathrm{V} \_$

Dp_i $=D p \_i * 10^{\wedge}$ interval

Endfor

while $\left(\mathrm{Dp}_{-} \mathrm{i}<0.400\right)$

printf "N_SMPS $=\% 8.0 \mathrm{fm}-3, \quad$ S_SMPS $=\% 6.0 \mathrm{~nm} 2 \mathrm{~cm}-3, \quad$ V_SMPS $=\% 4.0 \mathrm{fmm} 3 \mathrm{~cm}-3 \mid \mathrm{r}$ ", N_SMPS,

S_SMPS, V_SMPS

string point $=$ num2str(pointnum)

make/ $\mathrm{N}=16 / 0$ \$("SDstats_"+MonDD+"_"+point)=

\{pointnum,tri_mode_parameters[0],tri_mode_parameters[1],tri_mode_parameters[2],tri_mode_parameters[3],tri_mode_pa rameters[4],tri_mode_parameters[5],tri_mode_parameters[6],tri_mode_parameters[7],tri_mode_parameters[8],N_total, S_total, V_total,N_SMPS, S_SMPS, V_SMPS\}

edit SDstats_labels; appendtotable \$("SDstats_"+MonDD+"_"+point)

End 
Function CalcCondensationCharTIme(DDMonYY_Time, MonDD)

//This function calculates the characteristic time for condensational growth (tau) of the SD at each time

//but the output is the inverse of tau in units of per hour for comparison with the air exchange rate string DDMonYY_Time, MonDD

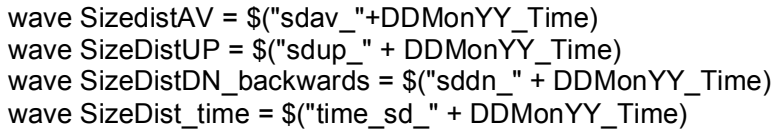

Variable length $=$ DimSize $($ SizedistAV,0) // number of size distributions in the matrix Variable Dpbins $=$ DimSize $($ SizedistAV,1) // number of Dp data points

duplicate/O SizedistDN backwards, SizedistDN

SizedistDN $=0$

SizedistDN = SizedistDN_backwards[p][Dpbins-1-q]

+ MonDD )

make/O/N=(length) \$("tau_inverse_up_" +MonDD ), \$("tau_inverse_dn_" +MonDD ), \$("tau_inverse_av_"

wave tau_inverse_up $=\$($ "tau_inverse_up_" +MonDD )

wave tau_inverse_av $=\$($ "tau_inverse_av_" +MonDD )

wave tau_inverse_dn $=\$($ tau_inverse_dn_" +MonDD )

wave dp_smps

variable $\mathrm{dp} \_$av, dlogDp, fuchs, Kn

variable diff $=0.1$

Variable j

tau_inverse_up $=0$; tau_inverse_dn $=0$; tau_inverse_av $=0$ for $(j=0 ; j<$ Dpbins $-1 ; j+=1)$

Dp_av $=\left(d p \_s m p s[j]+d p \_s m p s[j+1]\right) / 2 / /$ units $=n m$ dlogDp $=\log \left(d p \_s m p s[j+1] / d p \_s m p s[j]\right)$

$\mathrm{Kn}=2^{*} 65 / \mathrm{dp}$ av

fuchs $=2^{*} \mathrm{pi}^{*} \mathrm{dp} a \mathrm{v}^{*} \mathrm{diff}^{*} 10^{\wedge}-7^{*}(1+\mathrm{Kn}) /\left(1+1.71^{*} \mathrm{Kn}+1.333^{*} \mathrm{Kn} \mathrm{n}^{\wedge} 2\right)$

tau_inverse_up $=$ tau_inverse_up+SizedistUP[p][j] fuchs ${ }^{*}$ dlogDp* $3600 / /$ units of per hour

to match air exchange rate units

tau_inverse_av $=$ tau_inverse_av + SizedistAV[p][j] ${ }^{*}$ fuchs*dlogDp* 3600

endfor

tau inverse $d n=$ tau inverse $d n+$ SizedistDN[p][j]* fuchs ${ }^{*}$ dlogDp 3600

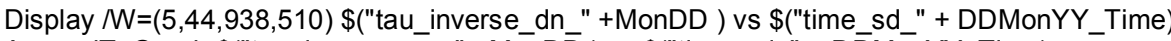

AppendToGraph $\$($ "tau_inverse_av_" +MonDD ) vs $\$($ "time_sd_" + DDMonYY_Time)

AppendToGraph \$("tau_inverse_up_" +MonDD ) vs \$("time_sd_" + DDMonYY_Time)

)$)=(1,4,52428)$

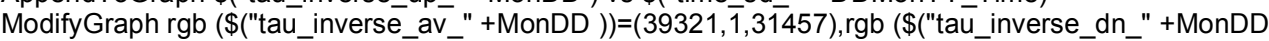

ModifyGraph $\log ($ left $)=1$; SetAxis left $0.1,10000$

ModifyGraph datelnfo(bottom) $=\{0,0,0\}$

Showinfo

End

Cursor/P A \$("tau_inverse_av_" +MonDD ) 100 


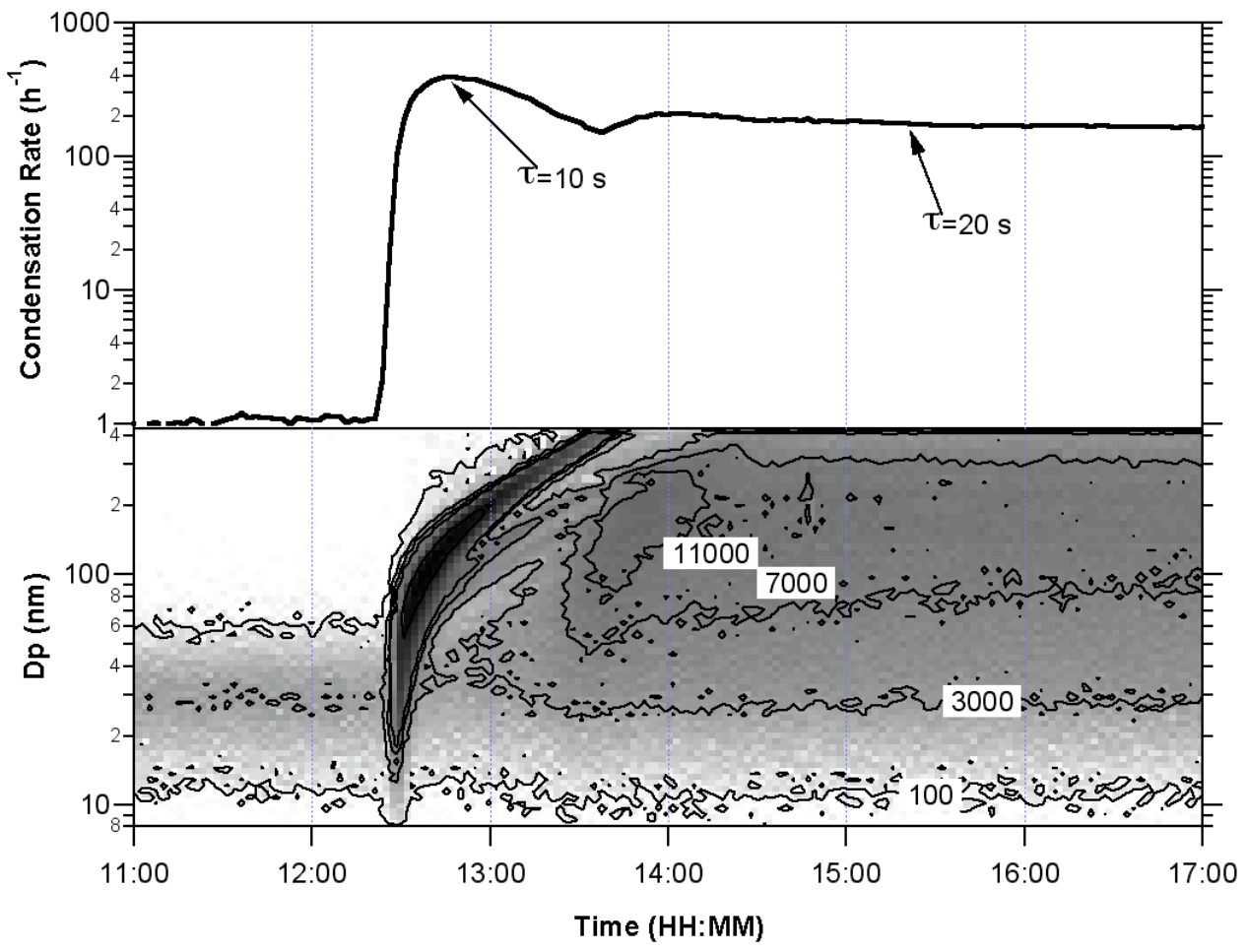

Figure 2A-1: Example output for function "CalcCondensationCharTime", which calculates the rate $\left(\mathrm{h}^{-1}\right)$ of vapor condensation to particle size distributions in a time series. The characteristic time $(\tau)$ is the inverse of the condensation rate constant. Experiment AFR-HH is shown. The lower frame shows particle measurements made using the SMPS, and contour lines indicate $\mathrm{dN} / \mathrm{d}\left(\log \mathrm{D}_{\mathrm{p}}\right)$ in $\mathrm{cm}^{-3}$. The shading scale is the same as in Figure 2-2. 


\section{B. Appendix: Additional experimental data}

Table 2B-1: Supply and residual terpene levels (all levels are given in ppb) for OOD experiments.

\begin{tabular}{lccc}
\hline & OOD-HH & OOD-HL & OOD-MH \\
\hline \hline $\begin{array}{l}\text { Supply level } \\
\text { limonene }\end{array}$ & 643 & 738 & 586 \\
$\begin{array}{l}\text { Residual level } \\
\text { limonene }\end{array}$ & 521 & 590 & 527 \\
& & & \\
$\begin{array}{l}\text { Total supply level } \\
\text { Total residual level }\end{array}$ & 543 & 738 & 586 \\
\hline
\end{tabular}

Table 2B-2: Supply and terpene residual levels (all levels are given in ppb) for AFR experiments.

\begin{tabular}{lccc}
\hline & AFR-HH & AFR-HL & AFR-MH \\
\hline \hline Supply level & & & \\
limonene & 121 & 165 & 108 \\
linalool & 176 & 247 & 170 \\
linalyl acetate & 66 & 88 & 57 \\
dihydromyrcenol & 241 & 335 & 242 \\
b-citronellol & 19 & 24 & 19 \\
& & & \\
Residual level & & & \\
limonene & 86 & 117 & 84 \\
linalool & 113 & 146 & 136 \\
linalyl acetate & 51 & 66 & 46 \\
dihydromyrcenol & 230 & 315 & 225 \\
b-citronellol & 11 & 14 & 14 \\
& & & \\
Total supply level & 623 & 859 & 596 \\
Total residual level & 492 & 658 & 506 \\
\hline
\end{tabular}


Table 2B-3: Supply and terpene residual levels (all levels are given in ppb) for POC experiments.

\begin{tabular}{|c|c|c|c|c|c|c|c|c|}
\hline & $\begin{array}{l}\sum^{\prime} \\
己 \\
0\end{array}$ & $\begin{array}{l}\underset{\Xi}{\Xi} \\
\underset{0}{\Xi}\end{array}$ & $\begin{array}{l}\stackrel{I}{I} \\
\text { 吾 } \\
0 \\
0\end{array}$ & 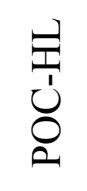 & 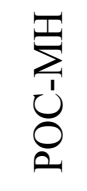 & 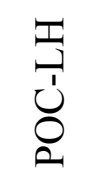 & $\begin{array}{l}O^{x} \\
Z \\
0 \\
0\end{array}$ & 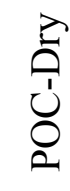 \\
\hline \multicolumn{9}{|l|}{ Supply level } \\
\hline limonene & 204 & 219 & 215 & 229 & 206 & 202 & 231 & 150 \\
\hline p-cymene & 40 & 41 & 40 & 42 & 37 & 49 & 47 & 27 \\
\hline eucalyptol & 53 & 58 & 55 & 60 & 46 & 53 & 61 & 51 \\
\hline terpinolene & 240 & 266 & 239 & 266 & 236 & 130 & 261 & 169 \\
\hline$\alpha$-terpineol & 146 & 149 & 117 & 104 & 154 & 104 & 174 & 131 \\
\hline$\gamma$-terpineol & 18 & 13 & 5 & 12 & 19 & 11 & 21 & 15 \\
\hline \multicolumn{9}{|l|}{ Residual level } \\
\hline limonene & 153 & 182 & 190 & 195 & 204 & 194 & 185 & 142 \\
\hline p-cymene & 41 & 41 & 42 & 41 & 38 & 49 & 43 & 28 \\
\hline eucalyptol & 55 & 56 & 51 & 58 & 55 & 54 & 56 & 51 \\
\hline terpinolene & 82 & 152 & 132 & 178 & 217 & 114 & 154 & 139 \\
\hline$\alpha$-terpineol & 112 & 128 & 114 & 113 & 157 & 123 & 162 & 139 \\
\hline$\gamma$-terpineol & 16 & 8 & 0 & 10 & 16 & 11 & 12 & 13 \\
\hline Total supply level & 723 & 771 & 692 & 738 & 722 & 565 & 817 & 558 \\
\hline Total residual level & 459 & 568 & 531 & 598 & 694 & 551 & 615 & 516 \\
\hline
\end{tabular}



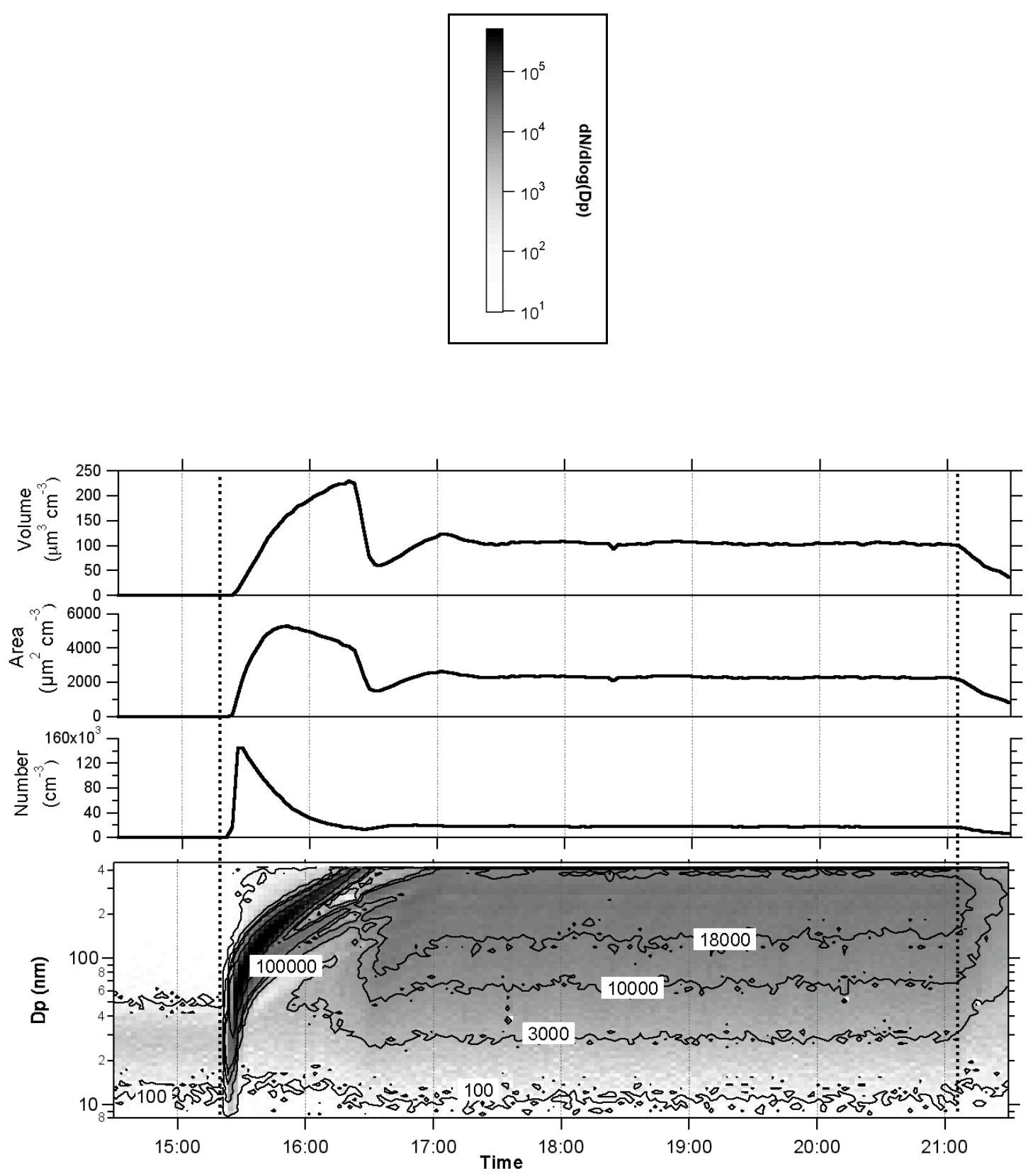

Figure 2B-1: Shading-scale for SMPS measurements (upper frame) and SMPS measurements for experiment OOD-HH (lower frame). Particle number concentration, $N$, is in units of $\mathrm{cm}^{-3}$. 


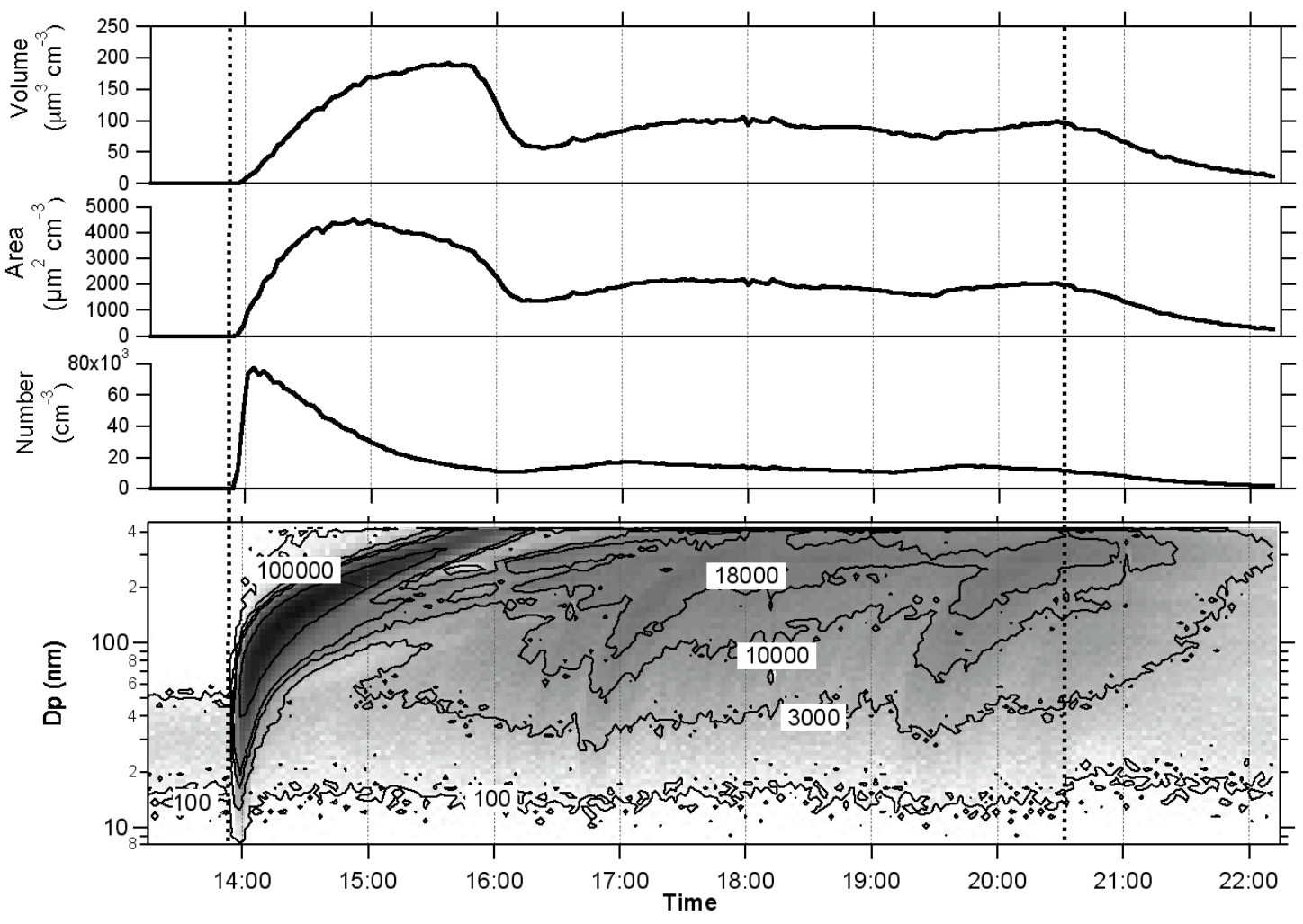

Figure 2B-2: SMPS measurements for experiment OOD-HL. The shading-scale for SMPS measurements is shown in Figure 2B-1. 


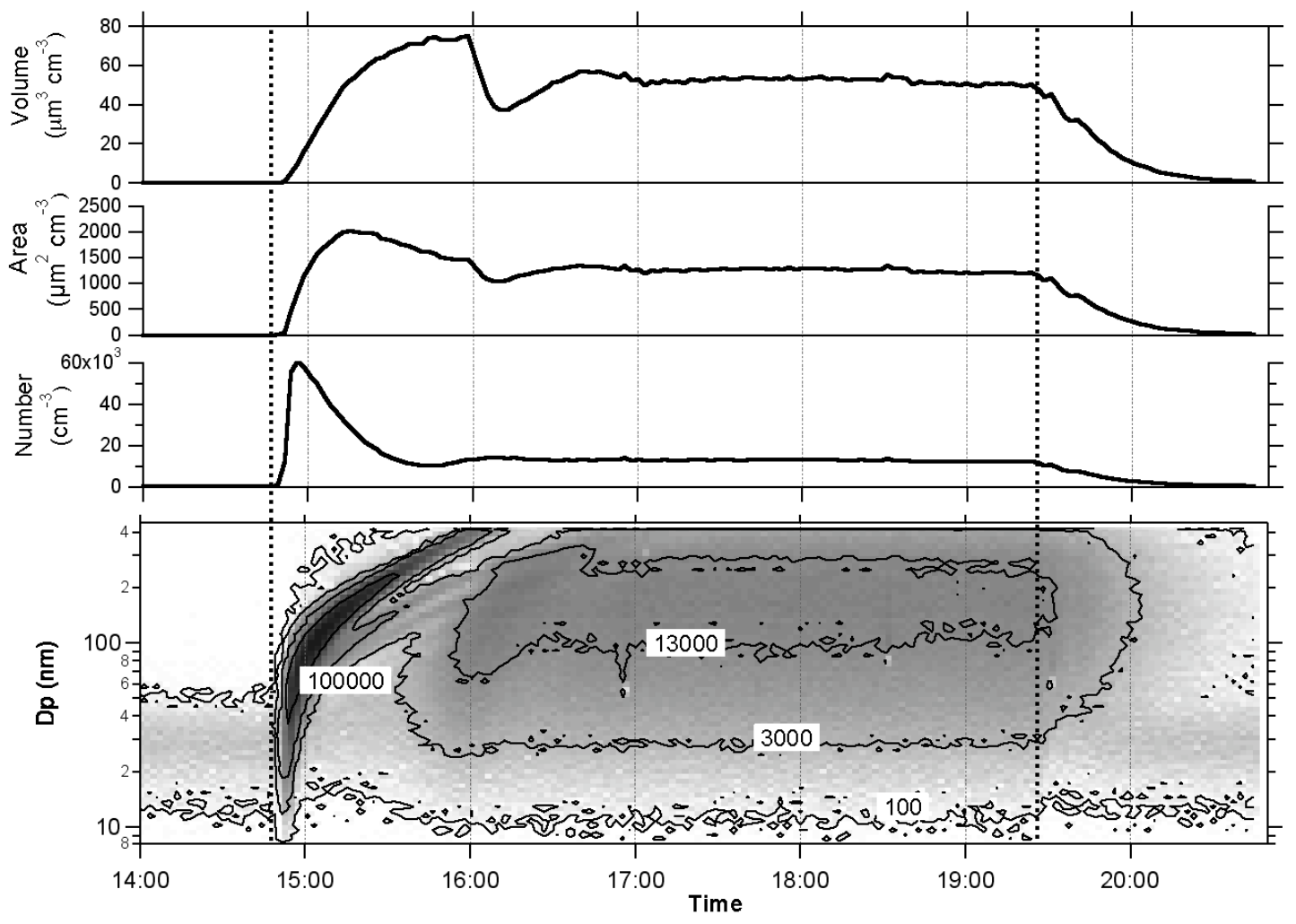

Figure 2B-3: SMPS measurements for experiment OOD-MH. The shading-scale for SMPS measurements is shown in Figure 2B-1. 

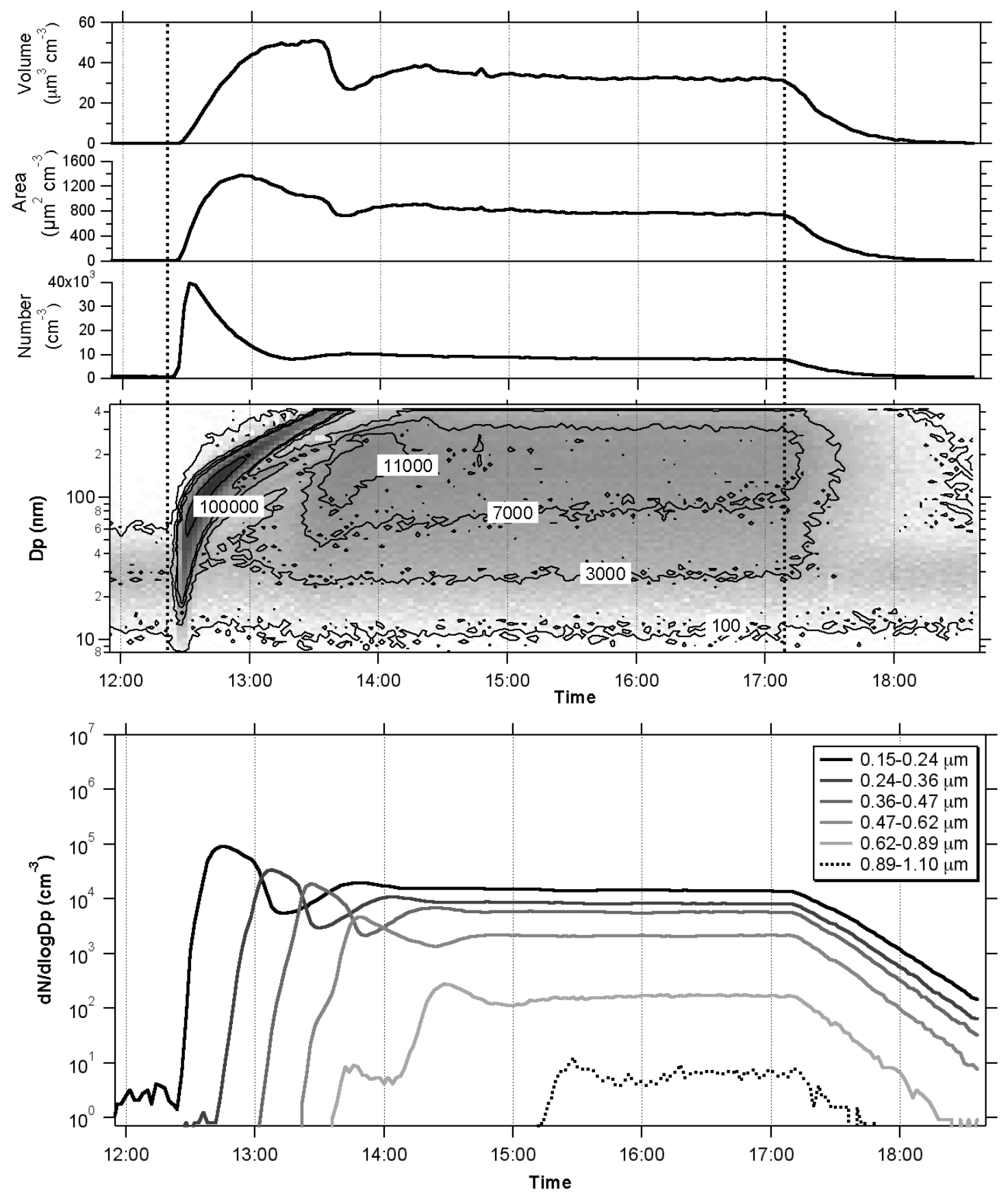

Figure 2B-4: SMPS (upper frame) and OPC (lower frame) measurements for experiment AFR-HH. The shading-scale for SMPS measurements is shown in Figure 2B-1. 

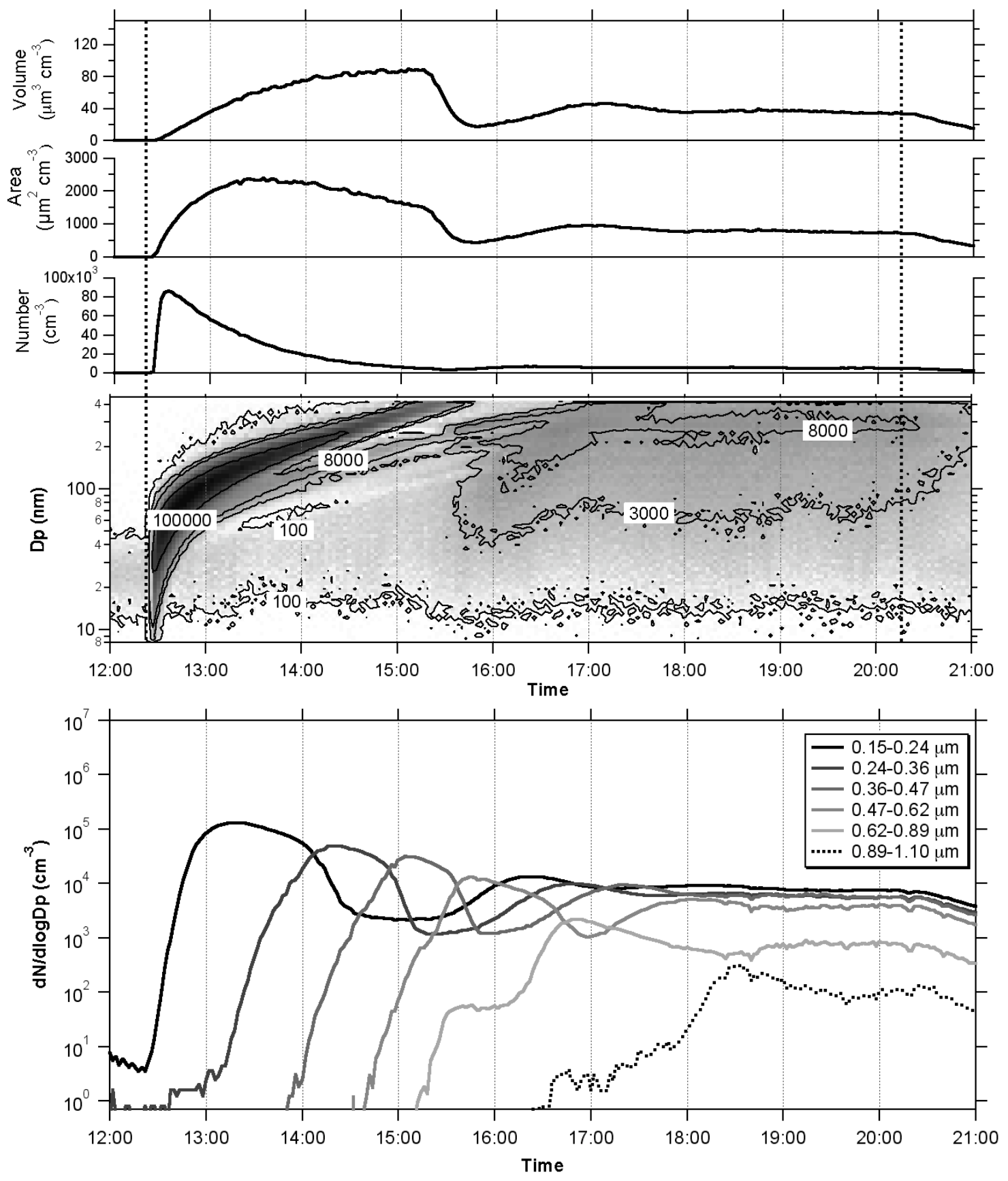

Figure 2B-5: SMPS (upper frame) and OPC (lower frame) measurements for experiment AFR-HL. The shading-scale for SMPS measurements is shown in Figure 2B-1. 

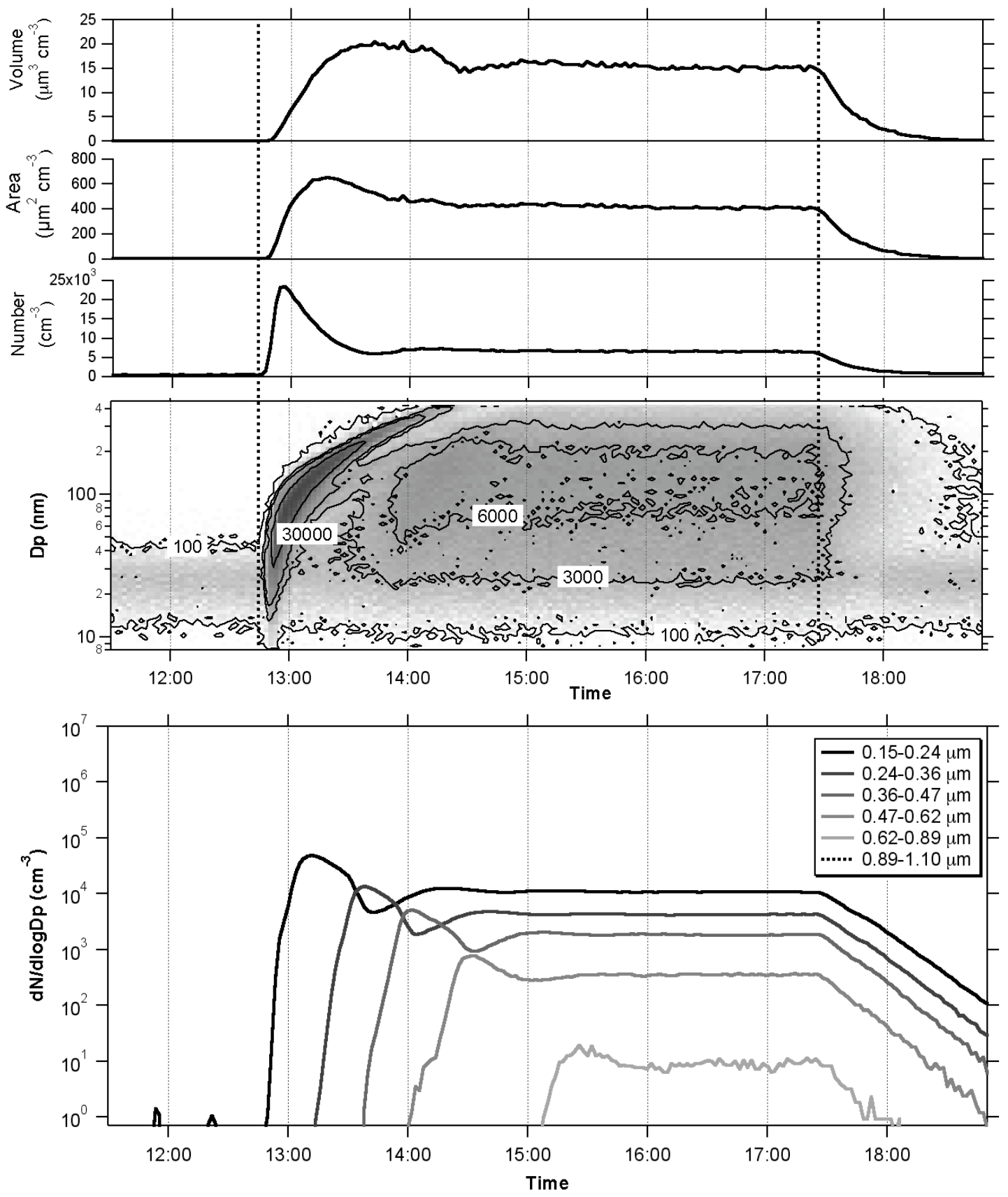

Figure 2B-6: SMPS (upper frame) and OPC (lower frame) measurements for experiment AFR-MH. The shading-scale for SMPS measurements is shown in Figure 2B-1. 

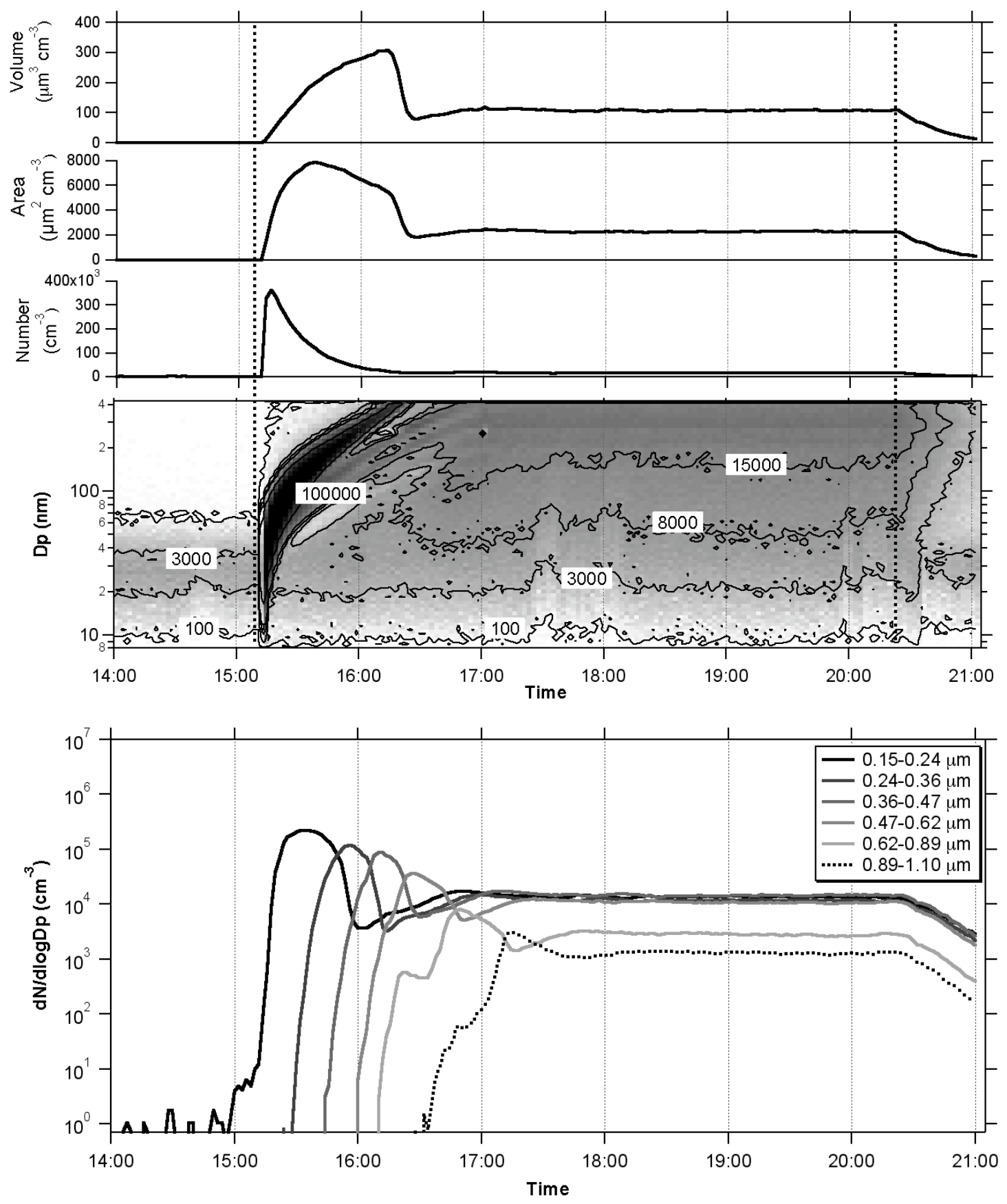

Figure 2B-7: SMPS (upper frame) and OPC (lower frame) measurements for experiment POC-VH. The shading-scale for SMPS measurements is shown in Figure 2B-1. 

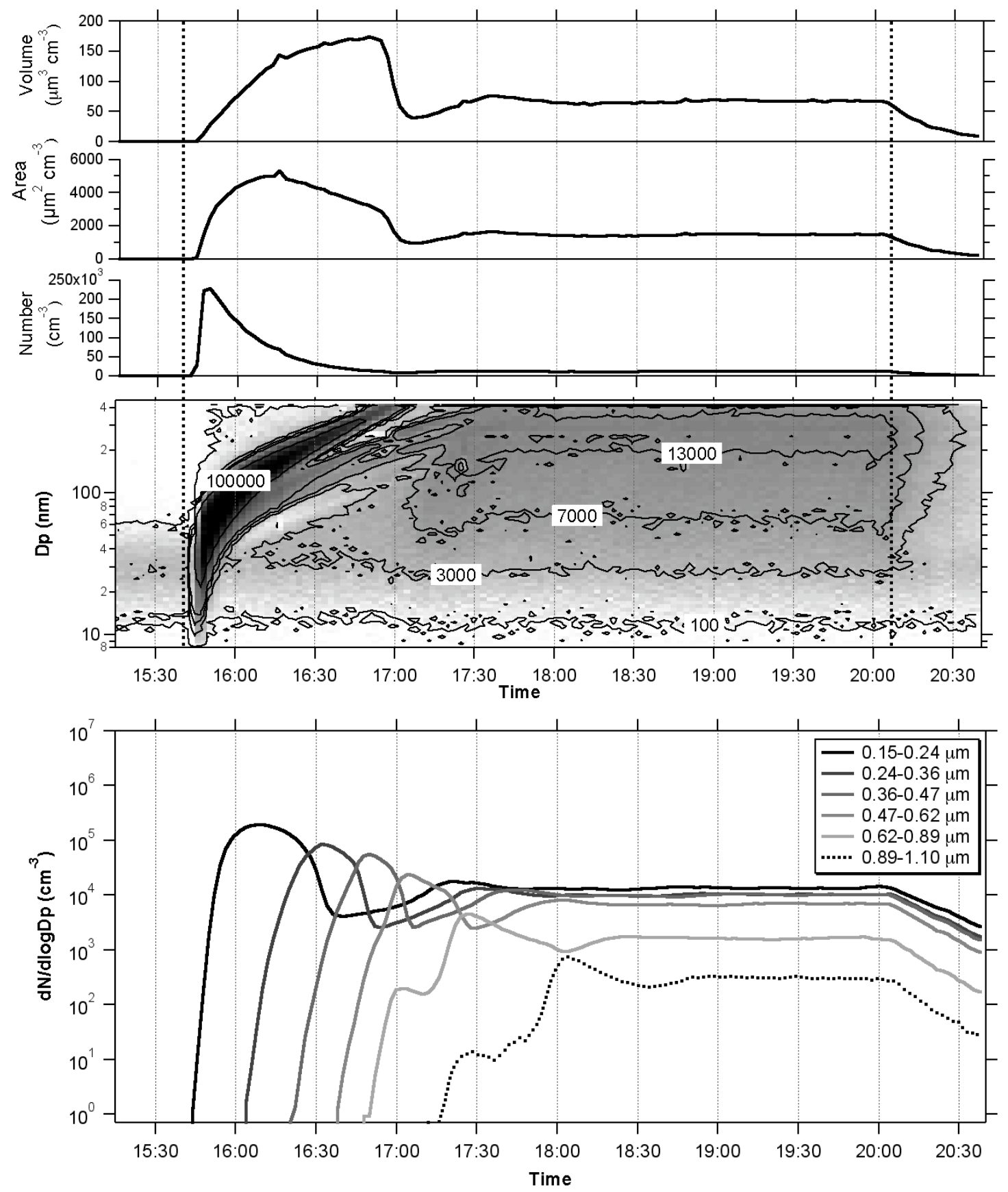

Figure 2B-8: SMPS (upper frame) and OPC (lower frame) measurements for experiment POC-HH1. The shading-scale for SMPS measurements is shown in Figure 2B-1. 


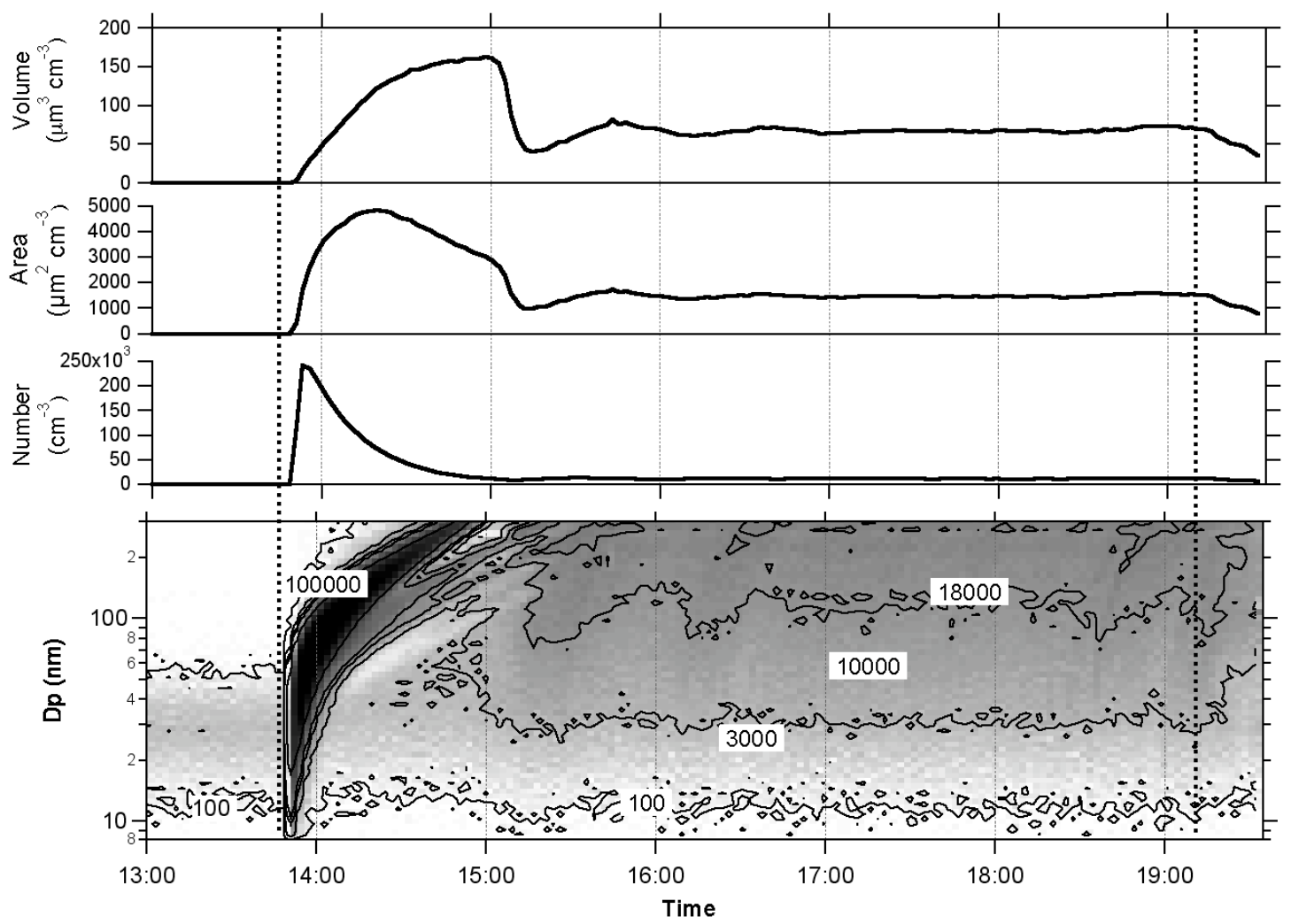

Figure 2B-9: SMPS measurements for experiment POC-HH2. The shading-scale for SMPS measurements is shown in Figure 2B-1. 


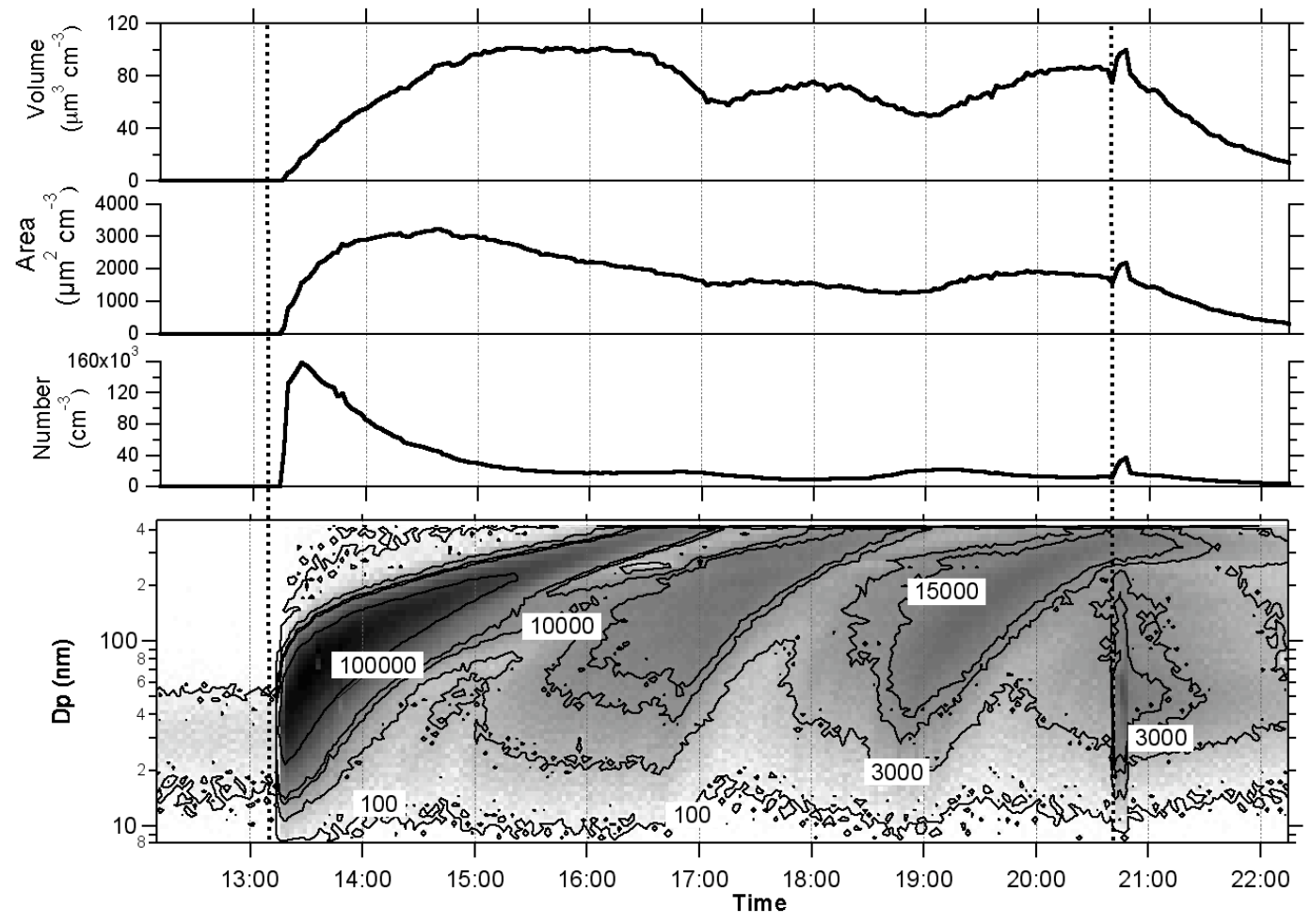

Figure 2B-10: SMPS measurements for experiment POC-HL. The shading-scale for SMPS measurements is shown in Figure 2B-1. 


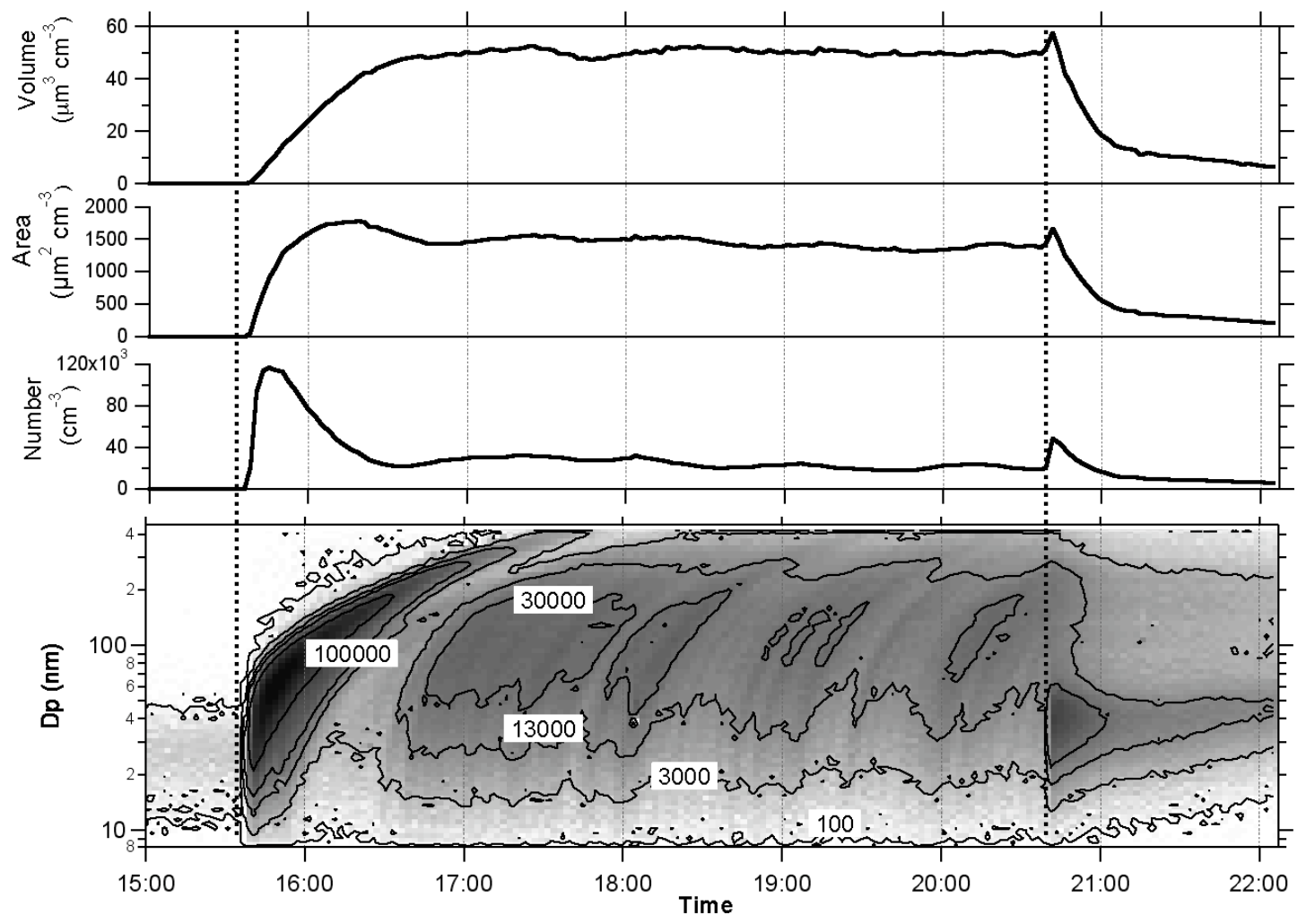

Figure 2B-11: SMPS measurements for experiment POC-MH. The shading-scale for SMPS measurements is shown in Figure 2B-1. 

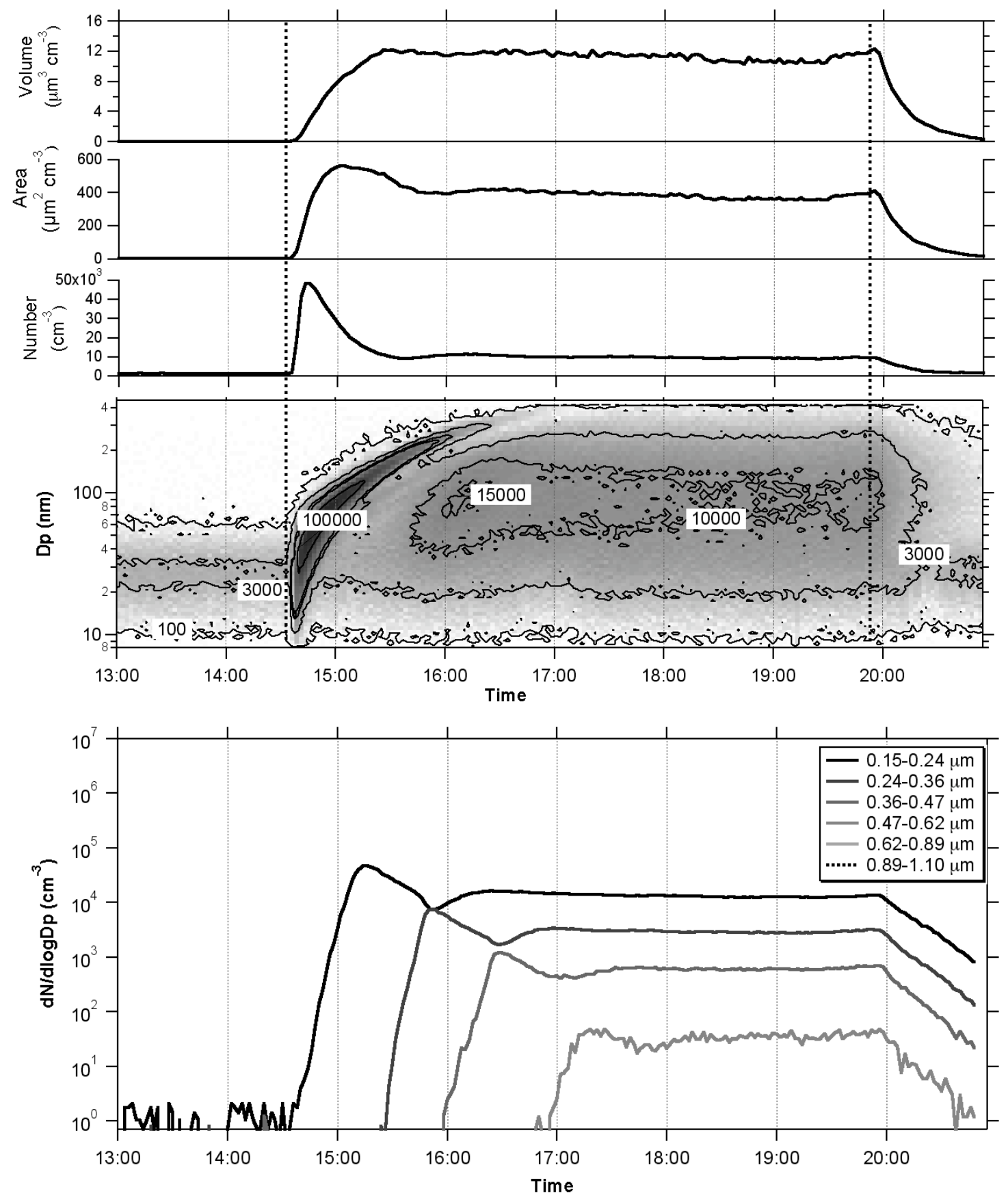

Figure 2B-12: SMPS (upper frame) and OPC (lower frame) measurements for experiment POC-LH. The shading-scale for SMPS measurements is shown in Figure 2B-1. 

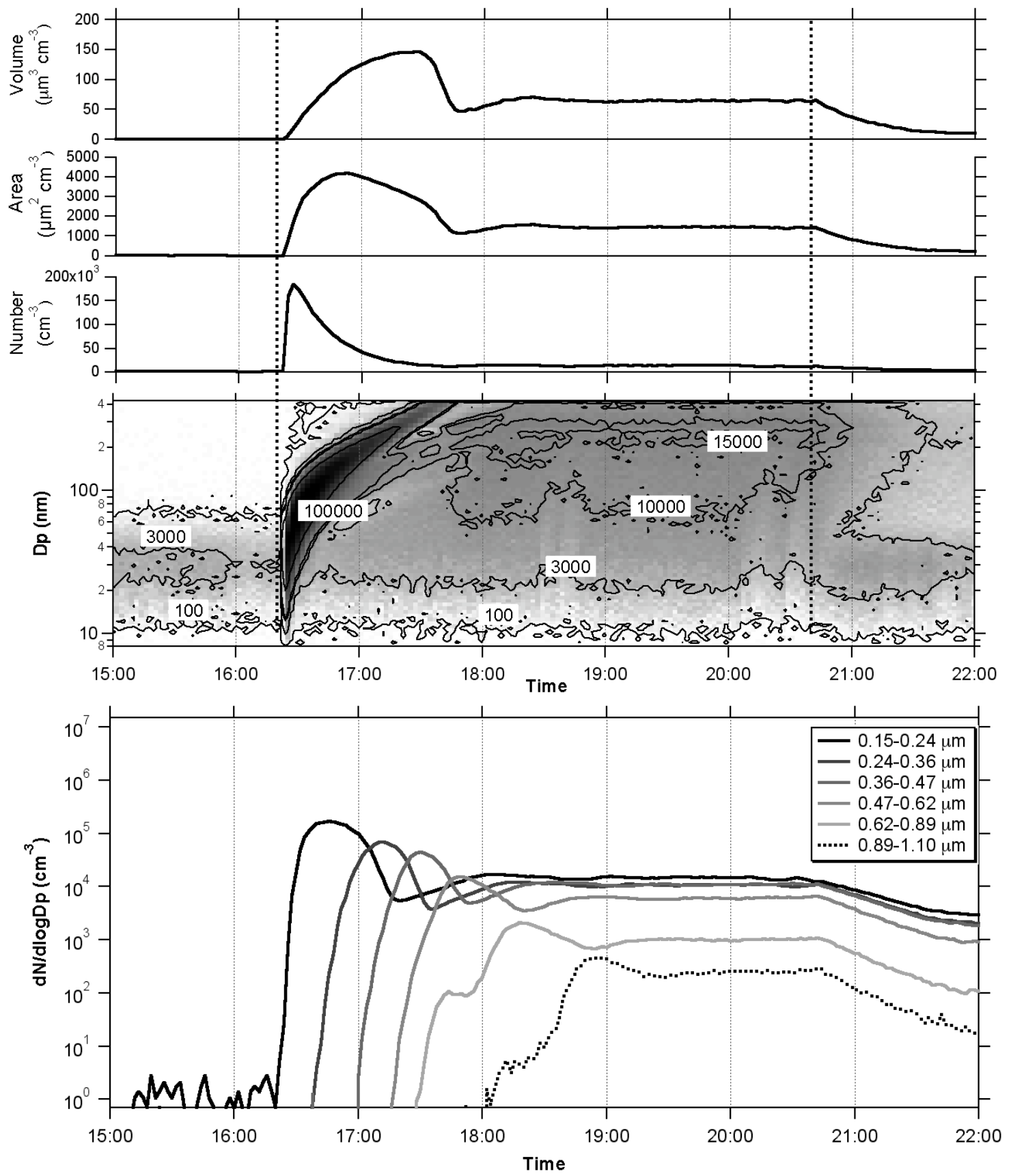

Figure 2B-13: SMPS (upper frame) and OPC (lower frame) measurements for experiment POC-NOx. The shading-scale for SMPS measurements is shown in Figure 2B-1. 

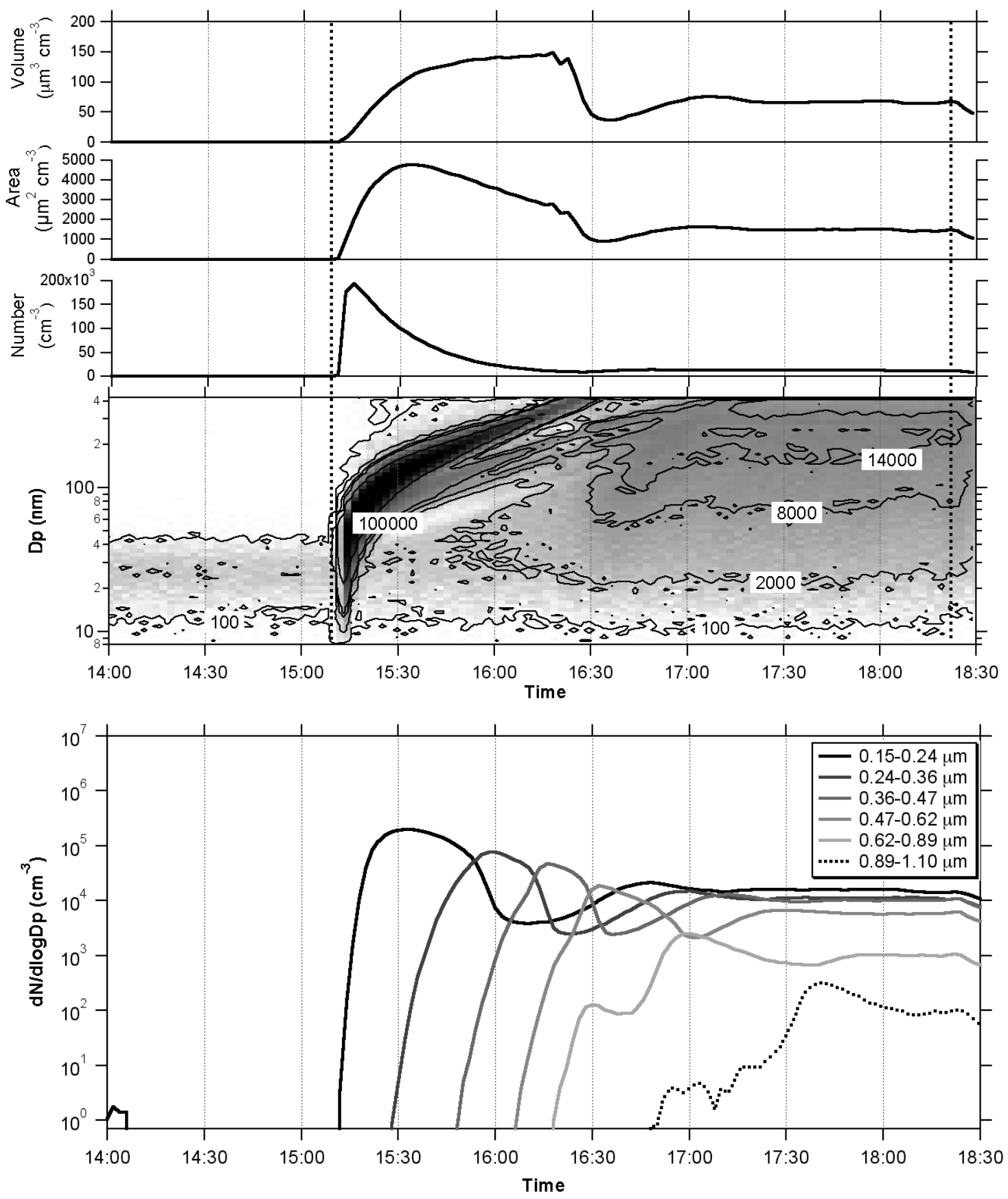

Figure 2B-14: SMPS (upper frame) and OPC (lower frame) measurements for experiment POC-Rev. The shading-scale for SMPS measurements is shown in Figure 2B-1. 

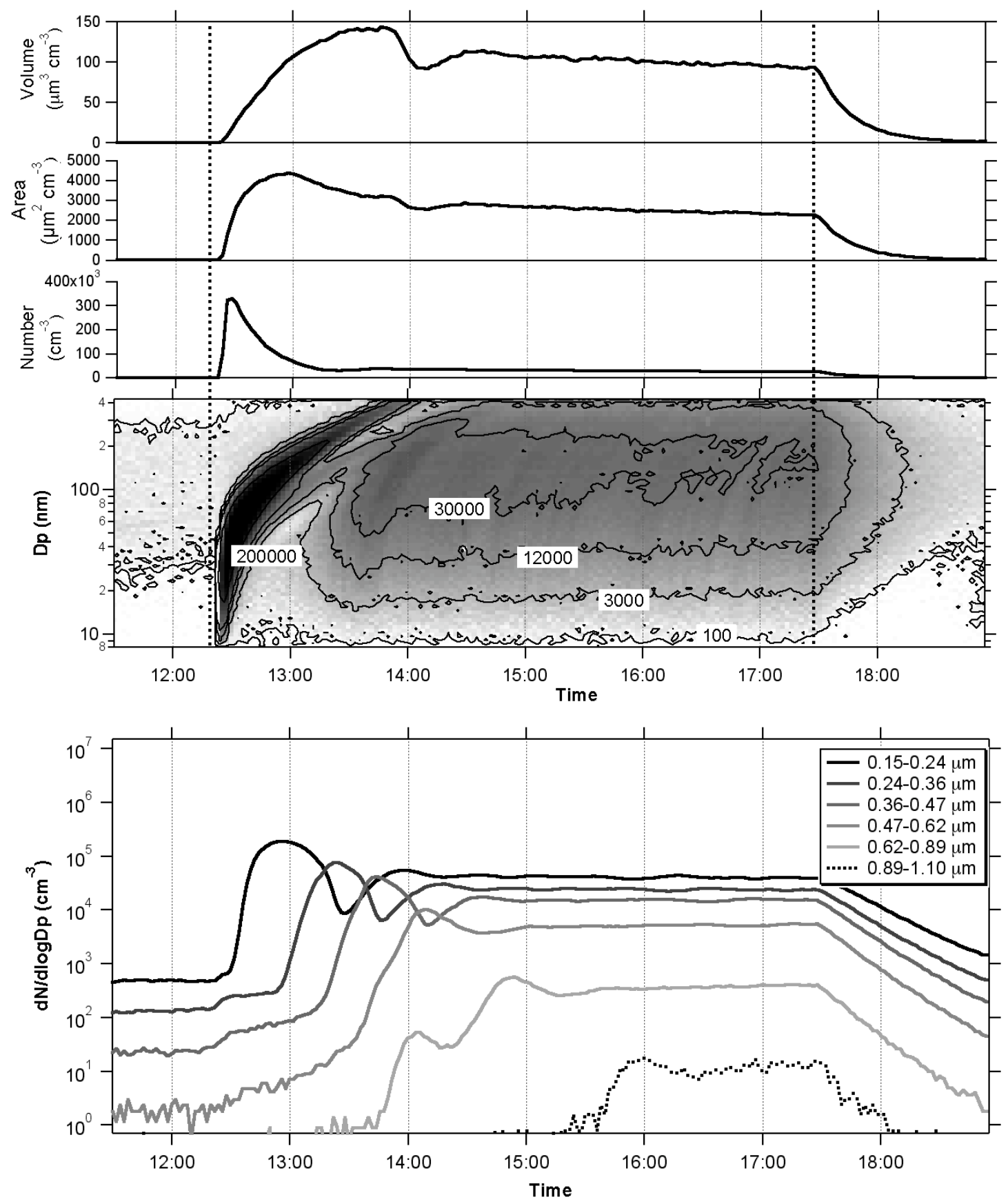

Figure 2B-15: SMPS (upper frame) and OPC (lower frame) measurements for experiment POC-Seed. The shading-scale for SMPS measurements is shown in Figure 2B-1. 


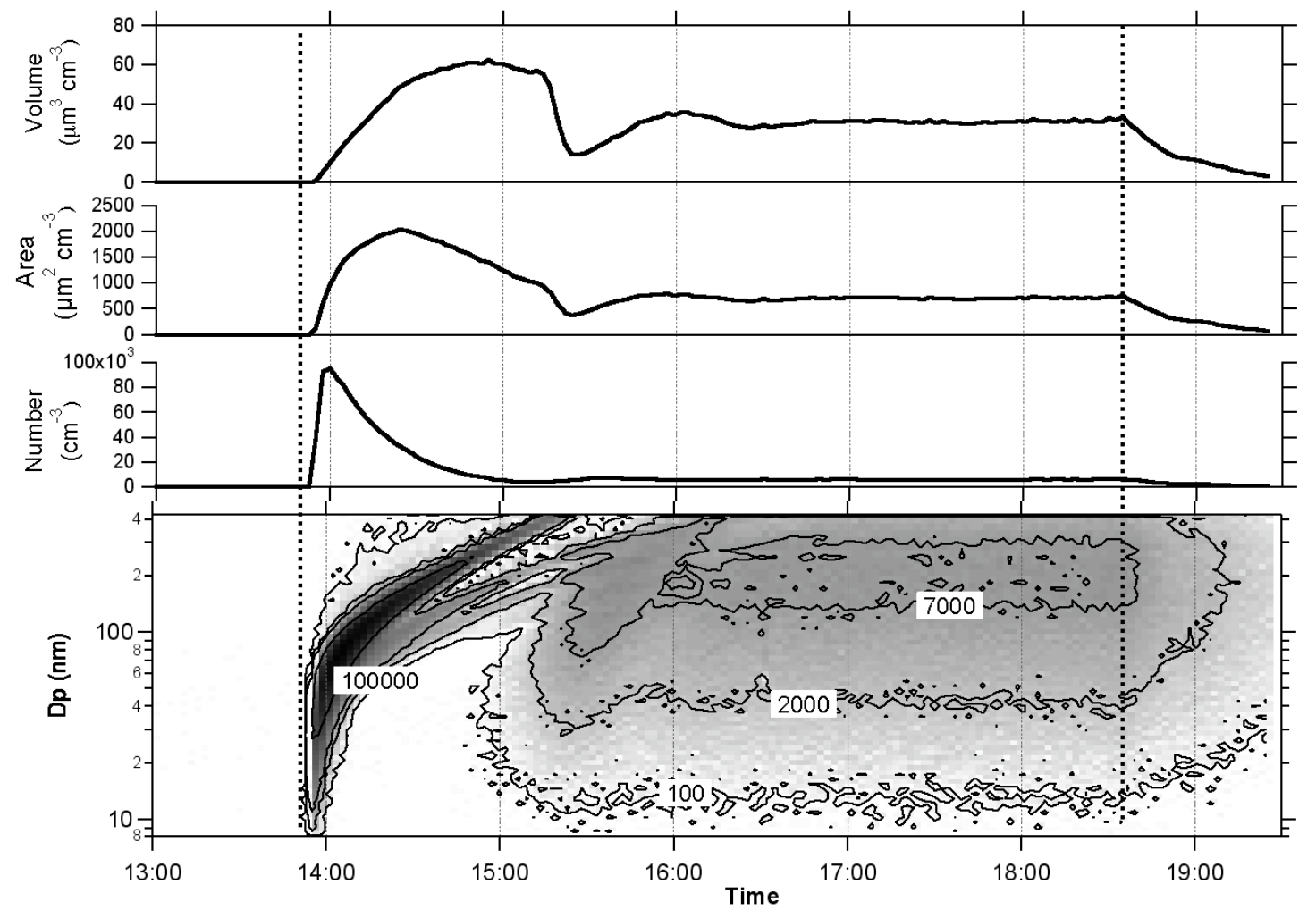

Figure 2B-16: SMPS measurements for experiment POC-Dry. The shading-scale for SMPS measurements is shown in Figure 2B-1. 

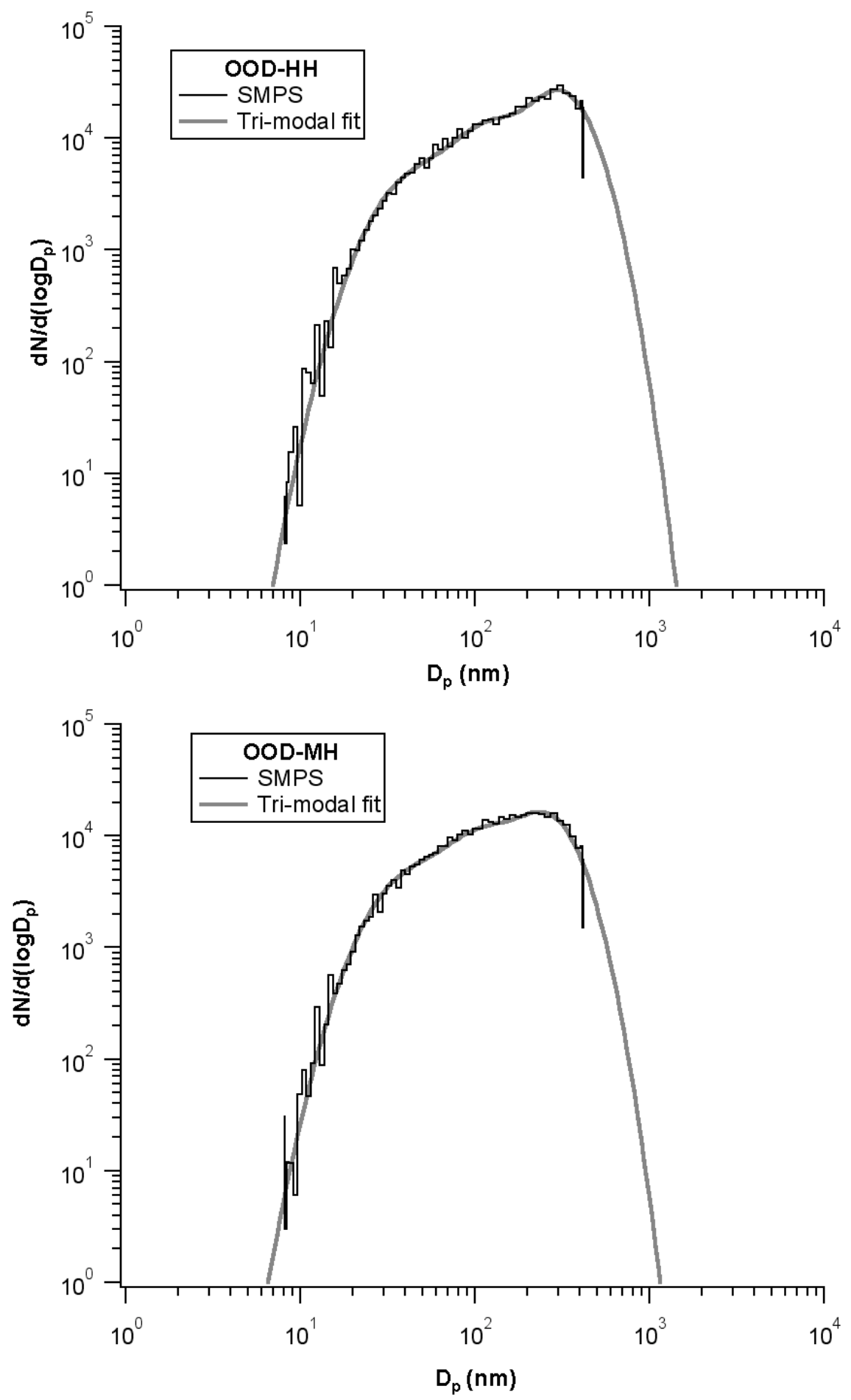

Figure 2B-17: Measured (with SMPS) and modeled (with tri-modal lognormal distributions) particle size distribution data from stage 4 (during "steady-state") of experiment OOD-HH (upper frame) and OOD-MH (lower frame). Particle number concentration, $N$, has units of $\mathrm{cm}^{-3}$. 

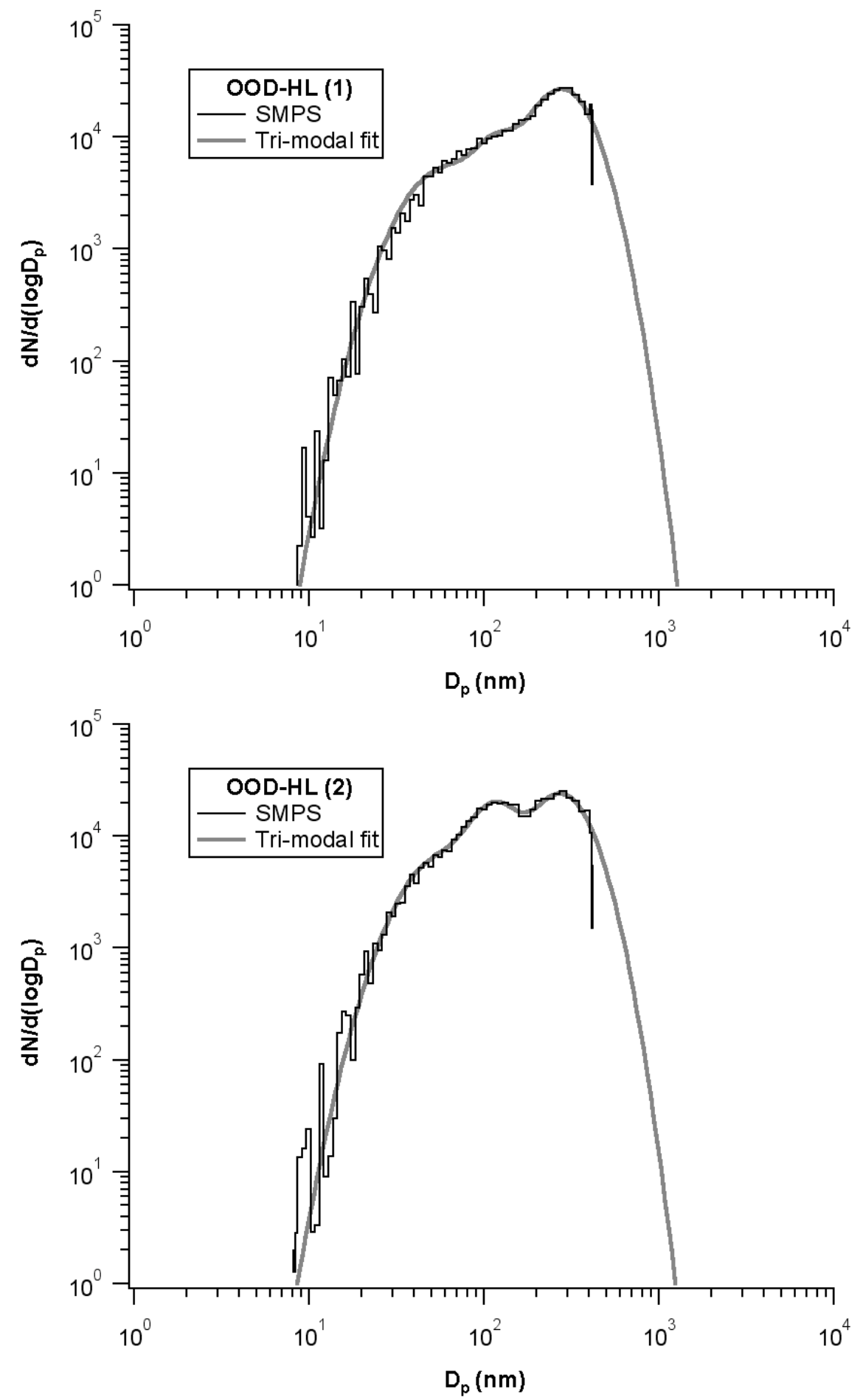

Figure 2B-18: Measured (with SMPS) and modeled (with tri-modal lognormal distributions) particle size distribution data from stage 4 of experiment OOD-HL. The particle distribution was "steady" in stage 4, so two distributions indicative of minimum and maximum particle concentrations are shown in the two frames. Particle number concentration, $N$, has units of $\mathrm{cm}^{-3}$. 

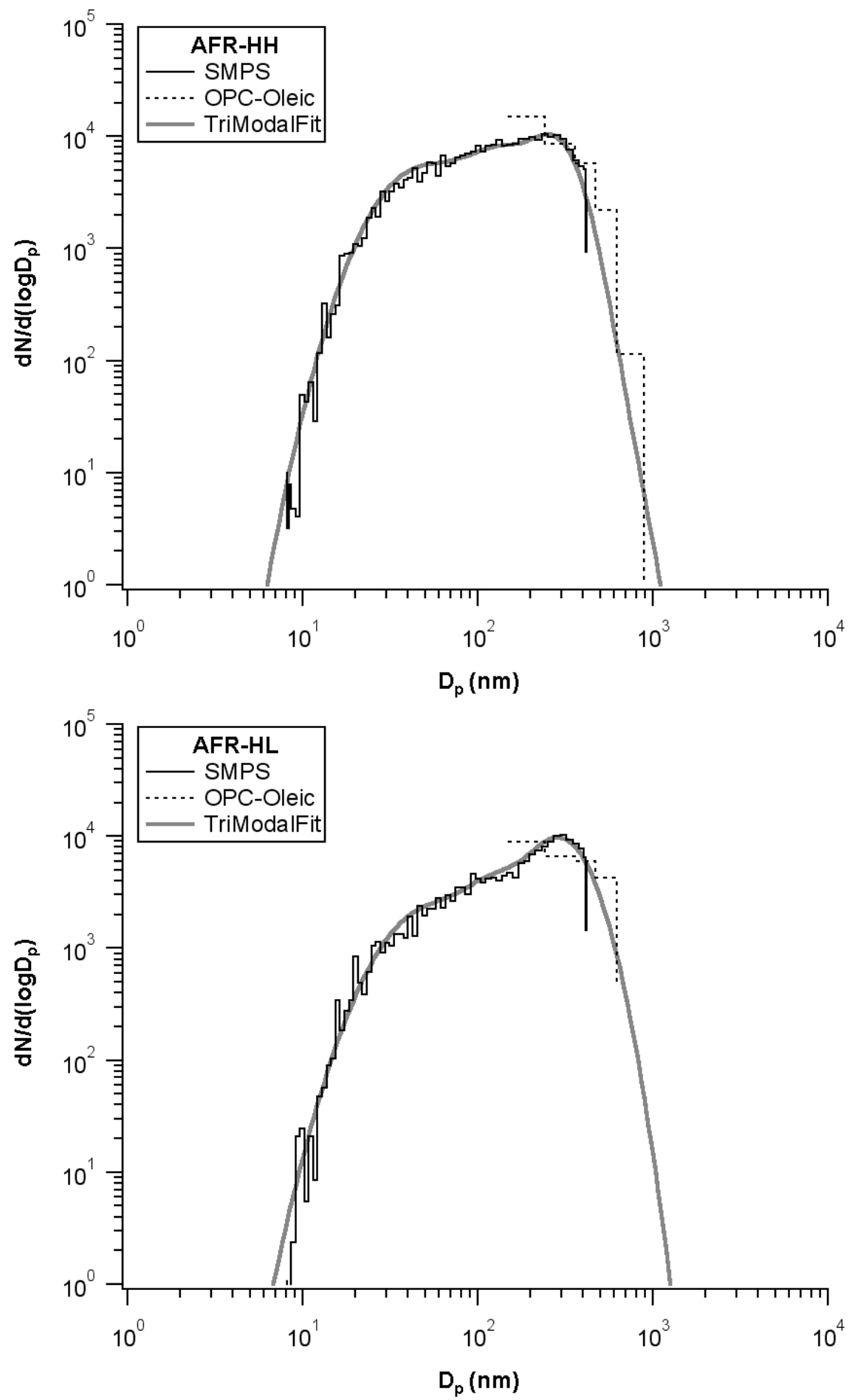

Figure 2B-19: Measured (with SMPS and OPC) and modeled (with tri-modal lognormal distributions) particle size distribution data from stage 4 (during "steady-state") of experiment AFR-HH (upper frame) and AFR-HL (lower frame). Particle number concentration, $N$, has units of $\mathrm{cm}^{-3}$. 

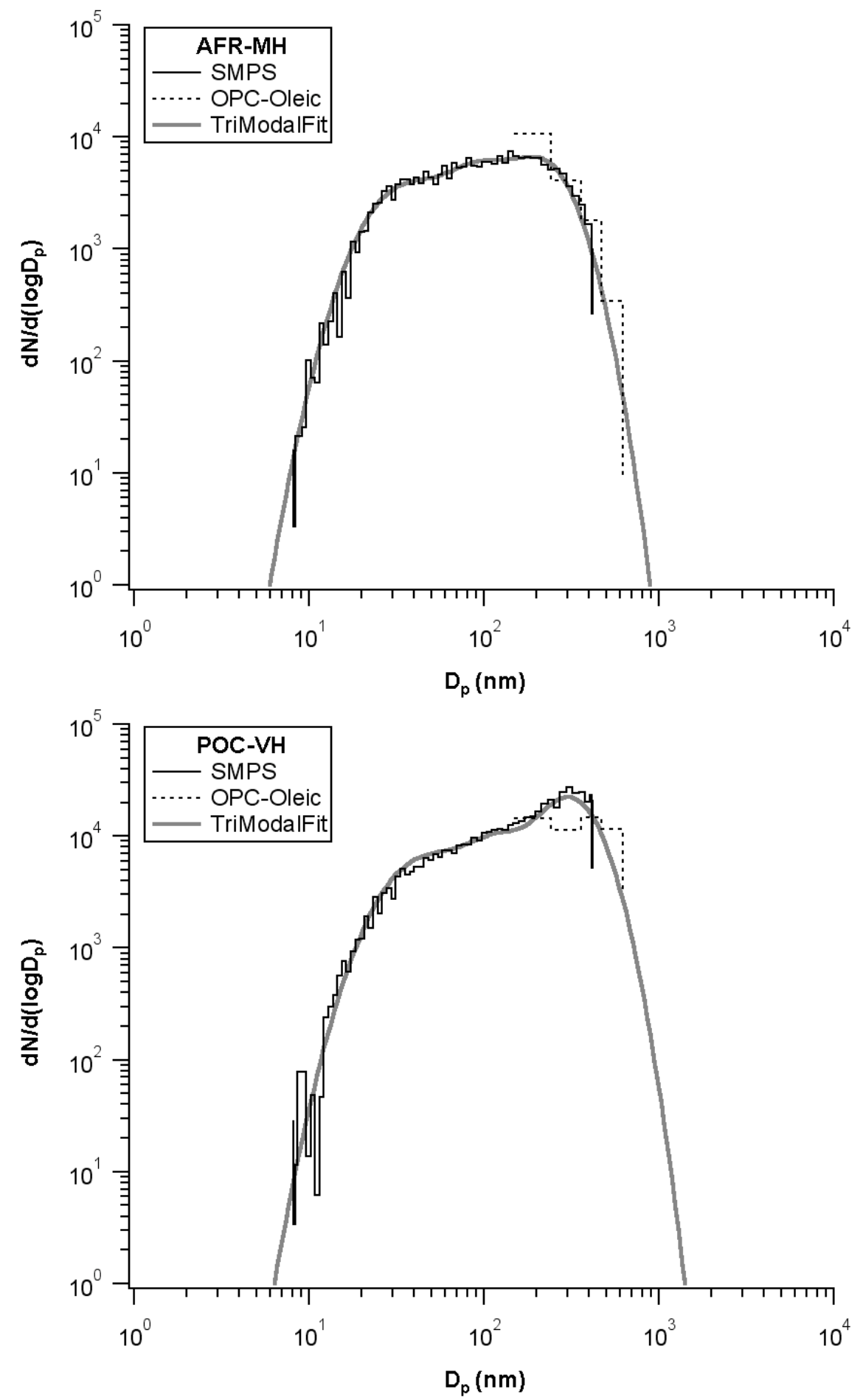

Figure 2B-20: Measured (with SMPS and OPC) and modeled (with tri-modal lognormal distributions) particle size distribution data from stage 4 (during "steady-state") of experiment AFR-MH (upper frame) and POC-VH (lower frame). Particle number concentration, $N$, has units of $\mathrm{cm}^{-3}$. 

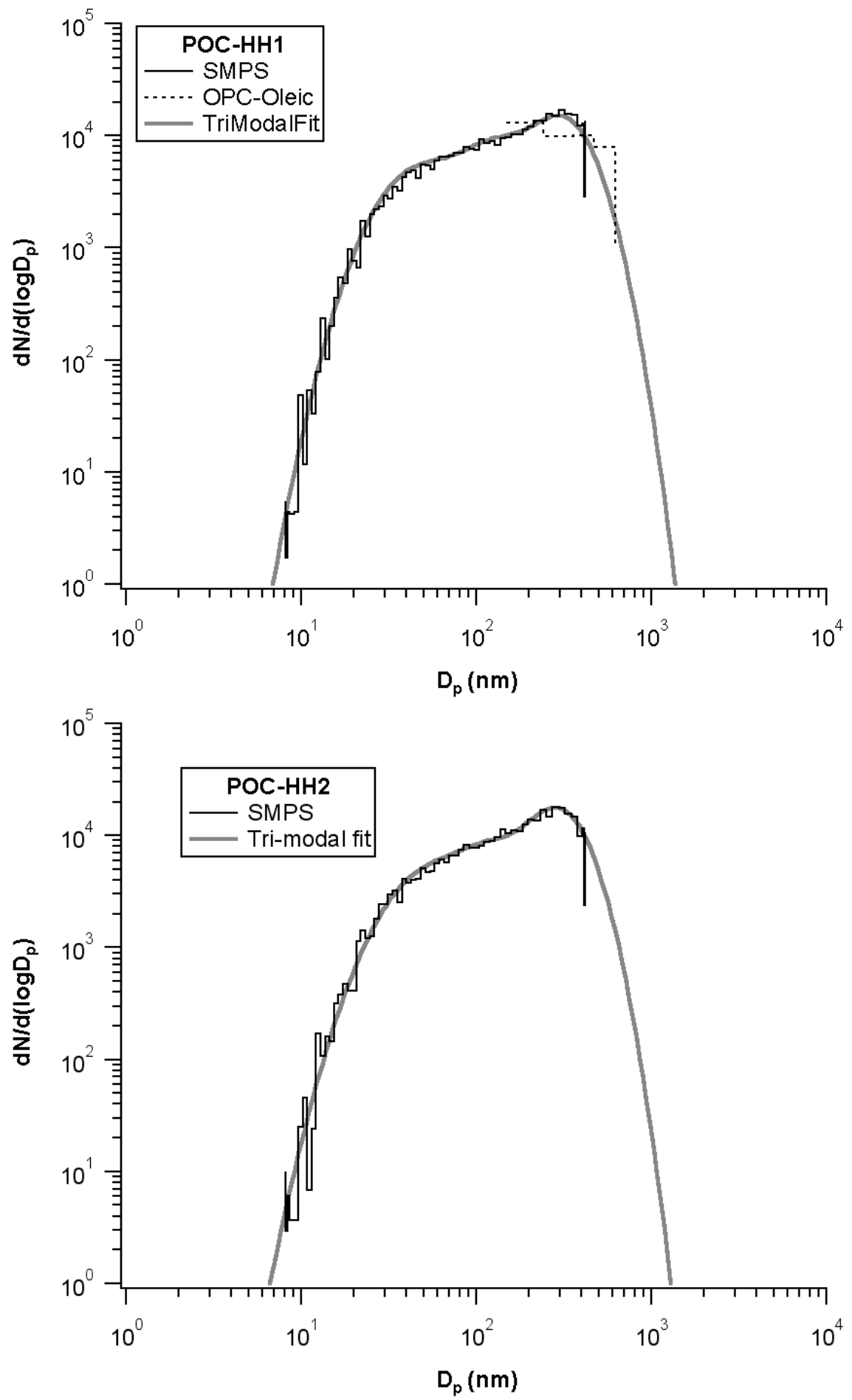

Figure 2B-21: Measured (with SMPS and OPC) and modeled (with tri-modal lognormal distributions) particle size distribution data from stage 4 (during "steady-state") of experiment POC-HH1 (upper frame) and POC-HH2 (lower frame). Particle number concentration, $N$, has units of $\mathrm{cm}^{-3}$. 

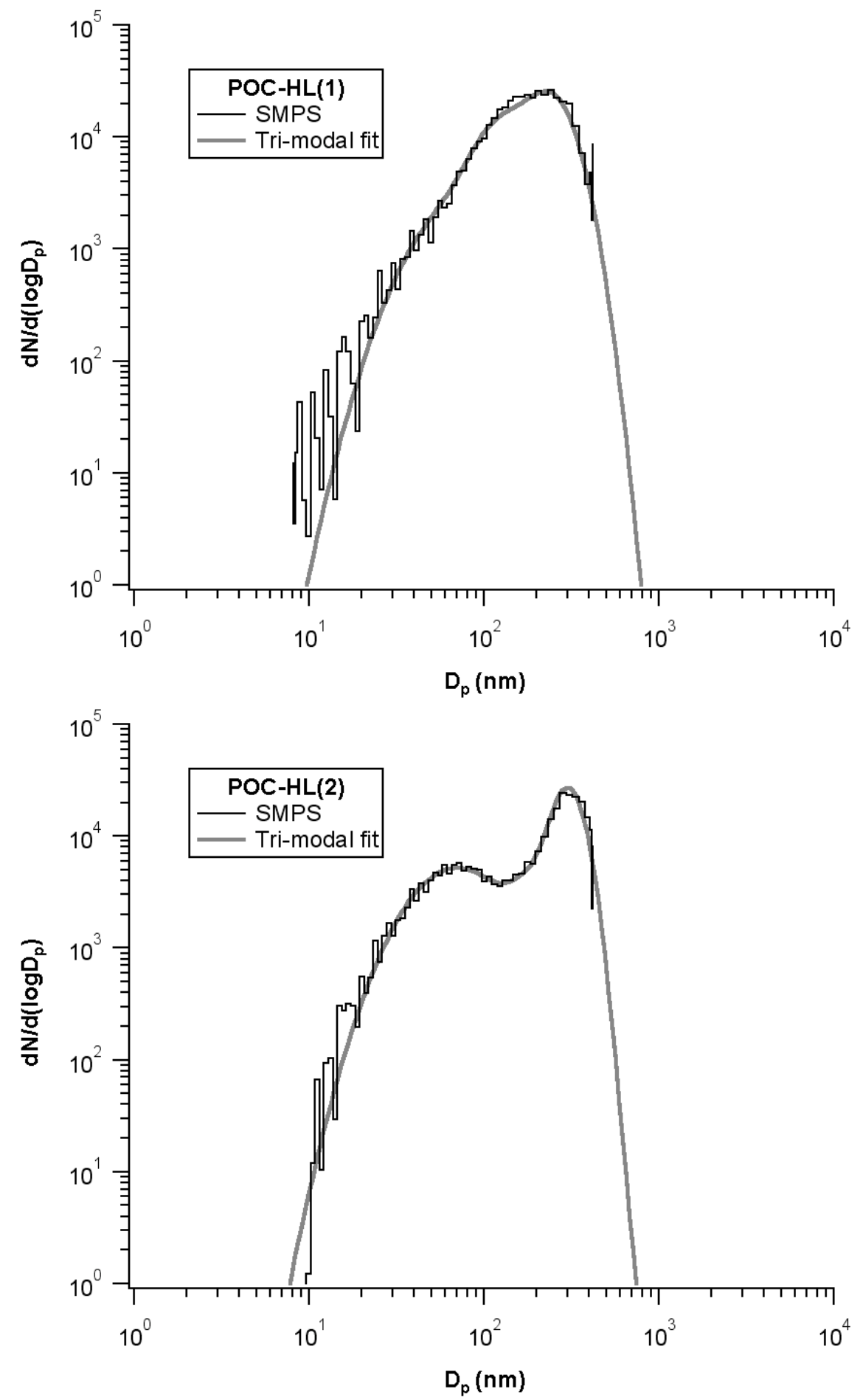

Figure 2B-22: Measured (with SMPS) and modeled (with tri-modal lognormal distributions) particle size distribution data from stage 4 of experiment POC-HL. The particle distribution was "steady" in stage 4, so two distributions indicative of minimum and maximum particle concentrations are shown in the two frames. Particle number concentration, $N$, has units of $\mathrm{cm}^{-3}$. 

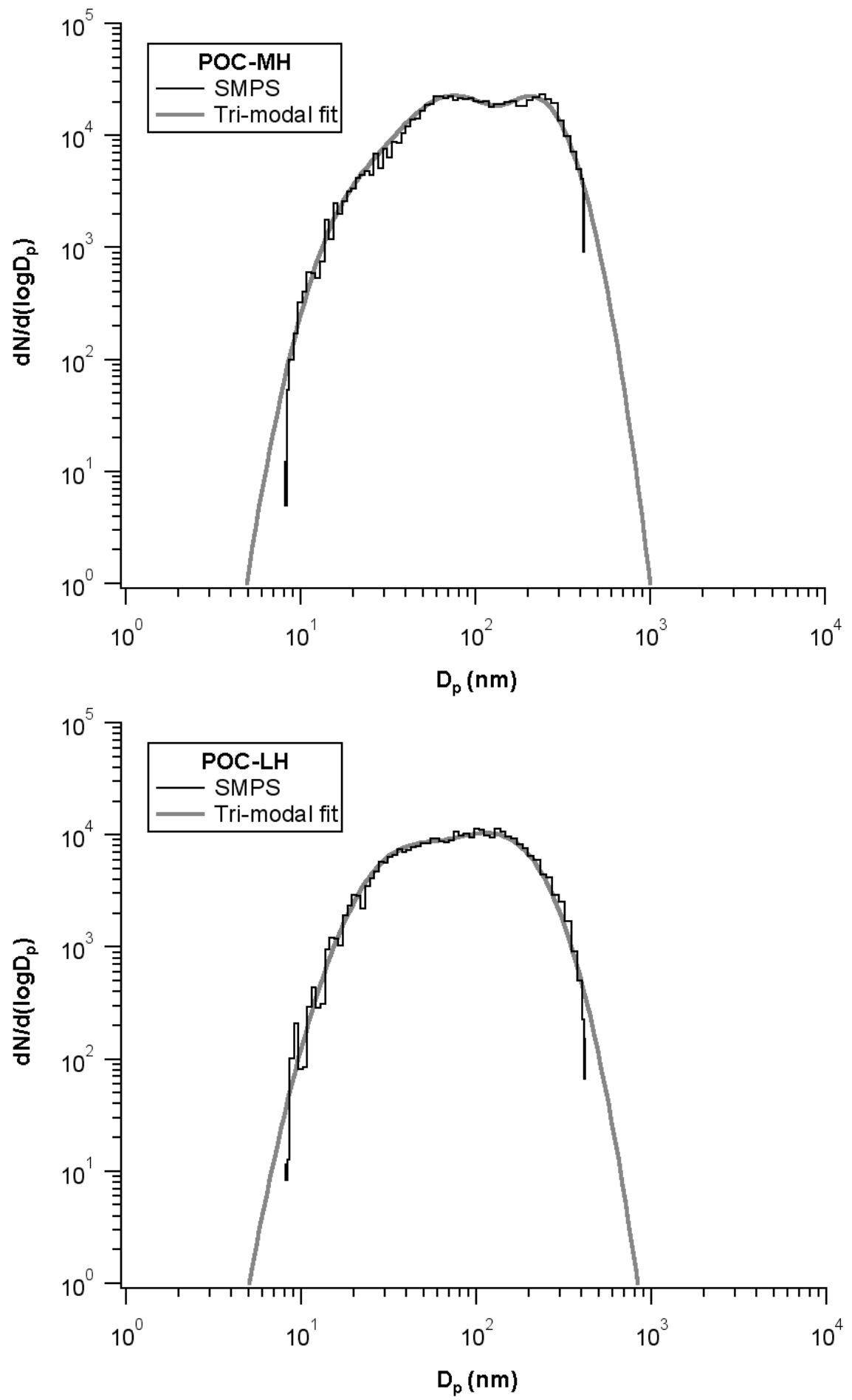

Figure 2B-23: Measured (with SMPS) and modeled (with tri-modal lognormal distributions) particle size distribution data from stage 4 (during "steady-state") of experiment POC-MH (upper frame) and POC-LH (lower frame). Particle number concentration, $N$, has units of $\mathrm{cm}^{-3}$. 

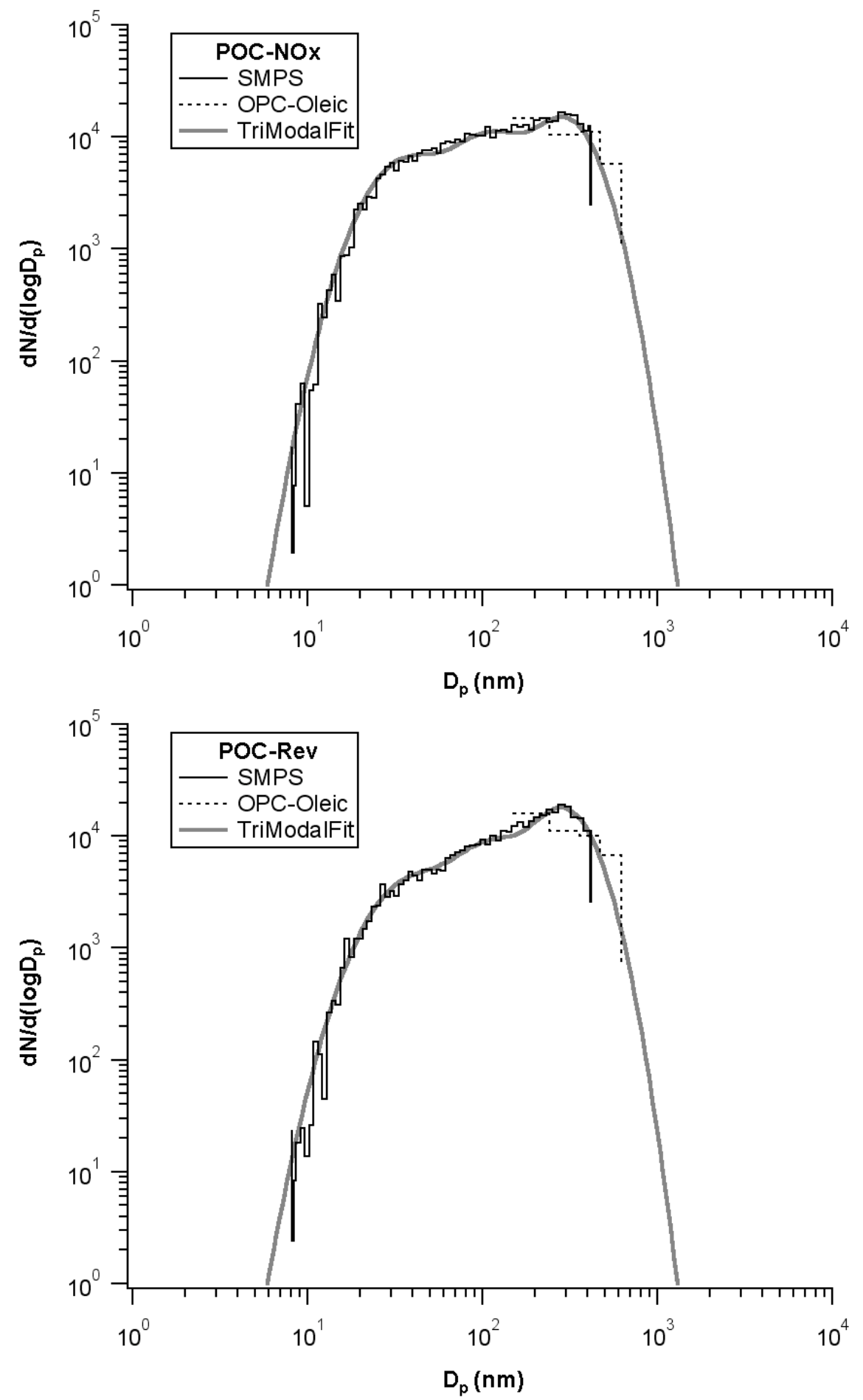

Figure 2B-24: Measured (with SMPS and OPC) and modeled (with tri-modal lognormal distributions) particle size distribution data from stage 4 (during "steady-state") of experiment POC-NOx (upper frame) and POC-Rev (lower frame). Particle number concentration, $N$, has units of $\mathrm{cm}^{-3}$. 

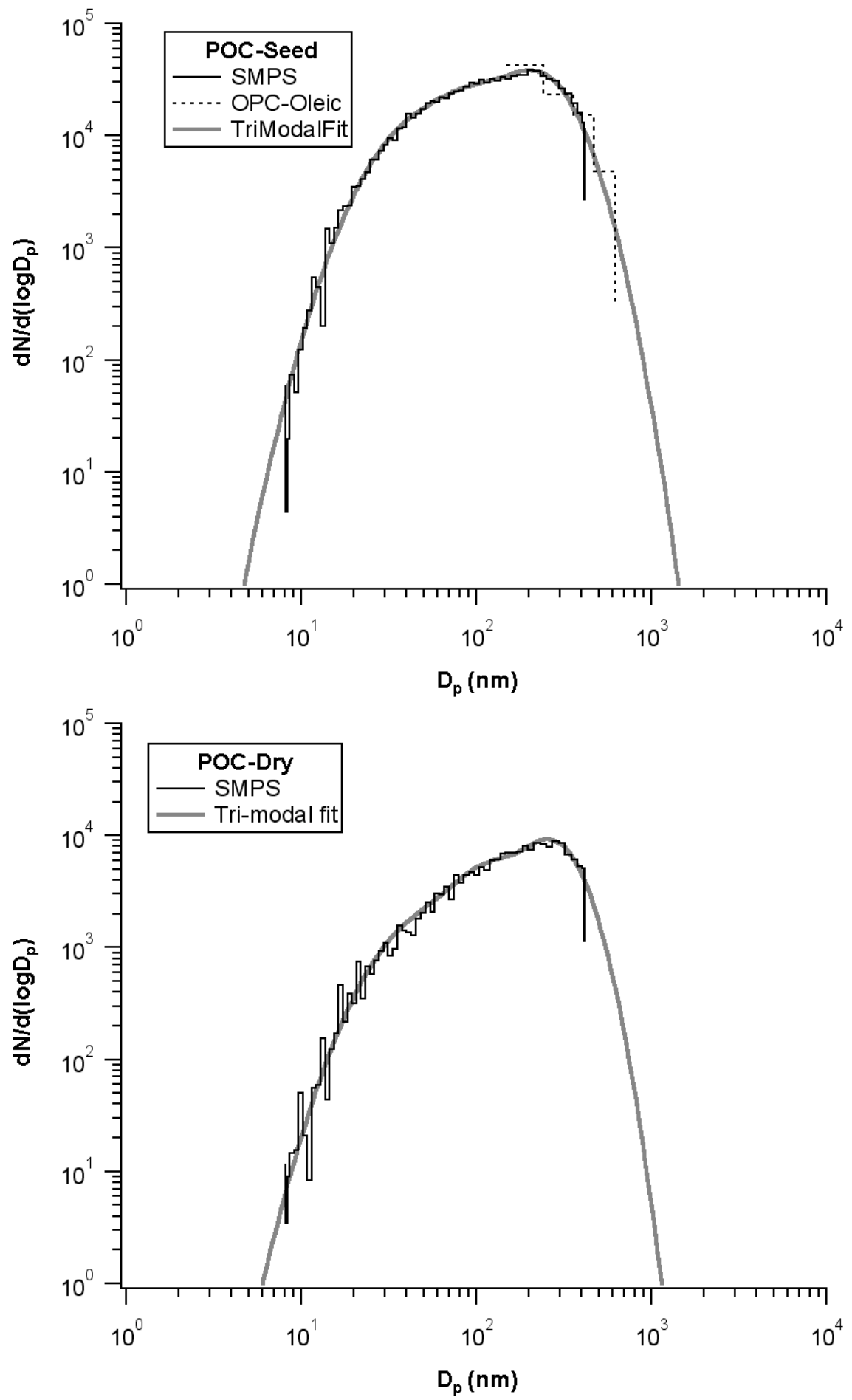

Figure 2B-25: Measured (with SMPS and OPC) and modeled (with tri-modal lognormal distributions) particle size distribution data from stage 4 (during "steady-state") of experiment POC-Seed (upper frame) and POC-Dry (lower frame). Particle number concentration, $N$, has units of $\mathrm{cm}^{-3}$. 


\section{Ozone-surface reactions, byproduct formation, and byproduct exposure in the aircraft cabin environment}

Reproduced in part with permission from Atmospheric Environment 42, 642-654, 2008. Copyright 2008, Elsevier Inc.

\subsection{Introduction}

Typical cruising altitude for commercial passenger flights is 8.8 to $12.5 \mathrm{~km}$, which is within the range of typical tropopause heights. The tropopause (the transition between the troposphere and the stratosphere) corresponds to the height at which the ozone concentration starts to increase with increasing altitude. The process by which stratospheric ozone is formed is photooxidation of molecular oxygen $\left(\mathrm{O}_{2}\right)$, and ozone production depends two main inputs: high energy radiation (wavelength $<242 \mathrm{~nm}$ ) and $\mathrm{O}_{2}$ concentration. The ozone "layer" is actually a vertical distribution of ozone concentration, as shown in Figure 3-1. At the top, ozone production is limited by insufficient $\mathrm{O}_{2}$ because there is an exponential drop in atmospheric pressure moving away from earth. Ozone production ceases at lower altitudes because high energy radiation has been significantly filtered out by absorption above.

Tropopause height varies with season and latitude. The annual average height ranges from $8 \mathrm{~km}$ at the poles to $18 \mathrm{~km}$ at the equator (Seinfeld and Pandis, 1998). Figure 3-1 shows three examples of ozone profiles during spring at $46^{\circ} \mathrm{N}, 60^{\circ} \mathrm{N}$, and $67^{\circ} \mathrm{N}$ from Müller et al. (2003) with ozone layer and typical flight altitudes superimposed on the profiles. The height of the tropopause decreases with increasing latitude, and thus the 
concentration of ozone that planes encounter is likely to increase with increasing latitude and increasing altitude. The tropopause tends to be at its lowest during winter and spring, and storm events can create regional areas of elevated ozone (Appenzeller and Davies, 1992). Consequently, planes are expected to encounter elevated ozone during these times. The ozone level at cruising altitude can range up to hundreds of ppb (Newchurch et al., 2003). When the ozone level is high outside the plane, the ozone level may also be elevated in the cabin since airplanes are continuously ventilated at high air-exchange rates using the air from outside the airplane. Other than ozone, the air of the upper troposphere and lower stratosphere is virtually free of pollutants and water vapor.

As summarized in a National Research Council report (NRC, 2002), several investigations were published in the 1960s and 1970s, documenting that elevated ozone levels posing health concerns occurred in aircraft cabins on some flights, especially those flying at high altitudes, high latitudes, and during the late winter and spring months (Brabets et al., 1967; Bischof, 1973). As a result, the Federal Aviation Administration established cabin ozone concentration limits in 1980.

There are two standards for cabin ozone levels on commercial passenger flights. The Federal Aviation Regulations (FARs) state that the 3-h average ozone level cannot exceed $100 \mathrm{ppb}$ at cruising altitudes above $8.2 \mathrm{~km}$ (FAR 25.832), and cannot exceed 250 ppb at any time above $9.7 \mathrm{~km}$ (FAR 121.578). Presumably, ozone regulations are tied to flight altitudes because of the correlation between altitude and ozone concentration. Planes are not typically equipped with indoor and outdoor ozone monitors, so actual ambient and cabin are not known or accounted for during flight. (There is a large set of real-time ambient ozone measurements collected during flight in the Measurement of 
Ozone by Airbus In-Service Aircraft (MOZAIC) project (Law et al., 2000).) Instead, airlines rely on statistical ozone levels based on time of year, latitude, longitude, and altitude. They use a combination of route planning, i.e. designing flight paths to avoid high cabin ozone levels based on statistical ambient ozone levels, and ozone converters to destroy ozone before it enters the cabin when high ozone levels are encountered. Currently, not all planes have ozone converters and, even when present, there is no consistent protocol in place to ensure their effective performance (NRC, 2002). In addition, there is no regular monitoring protocol in place to ensure compliance with the ozone standards.

Until recently, there were very few studies on ozone in the cabin environment after the early 1980s. One study measured in-flight cabin ozone concentrations using passive samplers and reported an average level of $80 \mathrm{ppb}$ during 108 U.S. domestic, Pacific, and southeast Asia routes, suggesting that elevated ozone is still an issue of potential concern in aircraft cabins (Spengler et al., 2004). More recently, real-time, inflight measurements on U.S. domestic flights indicate that ozone levels of tens to lowhundreds of ppb are common on aircraft without ozone converters (Bhangar et al., 2008). This study found a flight-average ozone level of $20.1 \mathrm{ppb}$ (geometric mean, with geometric standard deviation of 2.3) on 46 US domestic, transcontinental flights without ozone converters.

The conditions in the cabin differ from other indoor environments in that the cabin environment is characterized by low relative humidity $(\sim 10-20 \%)$, high airexchange rate $\left(\sim 10-20 \mathrm{~h}^{-1}\right)$ and reduced cabin air pressure ( 0.8 atm) (NRC, 2002). In addition, the occupant density and surface-to-volume ratio in the cabin are high; on a full 
flight there may be only 1-2 $\mathrm{m}^{3}$ of cabin volume per occupant, including shared spaces. These features have potentially important effects for ozone dynamics in aircraft cabins.

As with other indoor environments, the ozone level inside the plane is lower than the level outside the plane because once ozone enters the space it is consumed by reactions, which principally occur on surfaces (Weschler, 2000). Studies conducted in a simulated aircraft cabin have confirmed that surfaces, including those associated with passengers, are the dominant contributors to ozone consumption and byproduct formation in airplane cabins (Wisthaler et al., 2005; Tamás et al., 2006; Weschler et al., 2007). While reactions with surfaces reduce the level of ozone in cabin air, the byproducts of those reactions may be irritating or toxic, in some cases more so than ozone itself (Weschler, 2004).

Ozone decomposition on surfaces has been characterized in indoor spaces such as homes and offices (as summarized by Weschler, 2000), and ozone deposition to common residential and commercial indoor materials have been studied in chamber experiments and modeled (e.g., Morrison and Nazaroff, 2002a; Reiss et al., 1995; Grøntoft and Raychaudhuri, 2004). Byproducts of ozone reactions with surfaces have been measured for some typical home furnishings. They include toxic air contaminants, such as formaldehyde and acetaldehyde, and compounds with low odor thresholds, such as hexanal, heptanal, nonanal, and various nonenal isomers (Weschler et al., 1992; Morrison and Nazaroff, 2002a; Wang and Morrison, 2006).

Recent studies of ozone-initiated chemistry in the cabin environment have revealed that occupants, particularly ozone reactions with their skin oil, are a significant sink of ozone and an important source of oxidation byproducts (Wisthaler et al., 2005; 
Tamás et al., 2006; Weschler et al., 2007). Skin oil contains unsaturated fatty acids and squalene, which are reactive with ozone (Nicolaides, 1974). The dominant, detected products of ozone-skin oil reactions include acetone, 6-methyl-5-hepten-2-one, geranyl acetone, 4-oxopentanal, nonanal, and decanal (Fruekilde et al., 1998; Pandrangi and Morrison, 2008).

In the airline industry, a parameter called the "retention ratio" is used to estimate what the cabin ozone level will be given the ozone level outside the plane. Retention ratio is essentially an indoor-to-outdoor ozone ratio for aircraft passenger cabins in the absense of active ozone control. The only known measurements of retention ratio, 0.465 and 0.825, are from a study conducted by Nastrom et al. (1980). Occupancy was not reported in that study and it is not clear how well the conditions studied then reflect today's aircraft cabin. By policy, aircraft can be assigned a default R-value of 0.7 for demonstrating compliance with FAA regulations (NRC, 2002).

Characterizing ozone uptake and byproduct formation by the various cabin surfaces, including passengers, is important for understanding exposure to ozone and to the products of the ozone-initiated chemistry in the aircraft cabin environment. Overall byproduct emissions in a simulated cabin have been measured, and byproduct emissions from some of the materials that are found in the cabin have been measured under conditions relevant to residential and commercial building environments. In the present study, ozone-surface reactions were investigated for individual materials common to the cabin environment at flight-relevant conditions. Experiments were carried out in a small chamber where cabin materials (seat fabric, carpet, and plastic) and clothing fabrics (polyester, wool, and cotton) were individually exposed to ozone at low relative humidity 
and high air-exchange rate. The experimental data were interpreted to quantify ozone deposition and uptake rate, to characterize formation of volatile organic byproducts of ozone-initiated chemistry, and to quantify byproduct emission rates and yields. The results presented in this chapter provide a new estimate of retention ratio based on ozone deposition to the individual materials in the cabin. In addition, data from this study and other recently published studies of cabin air quality are used to develop a model for predicting byproduct levels in the cabin. For a few oxidation byproducts, inhalation intakes in the cabin environment are estimated and are put into context by comparing them with intakes in other environments.

\subsection{Methods}

\subsubsection{Evaluating ozone deposition and byproduct emissions}

Formally, retention ratio, $R$, is defined as the mole fraction of ozone in the cabin air in the absence of deliberate control devices normalized by the mole fraction of ozone in the ambient air (NRC, 2002). If ozone deposition to surfaces dominates consumption, a steady-state mass balance applied to the aircraft interior results in the following model equation for $\mathrm{R}$.

$$
R \equiv \frac{\left[\mathrm{O}_{3}\right]_{\text {cabin }}}{\left[\mathrm{O}_{3}\right]_{\text {ambient }}} \cong \frac{\lambda}{\lambda+\sum_{i} v_{d, i} \frac{S_{i}}{V}}
$$

Here, $\lambda$ is the cabin air-exchange rate, $V$ is the volume of the cabin, $S_{i}$ is the nominal surface area of a given material $i, v_{d, i}$ is the deposition velocity of ozone to that material, and the summation is carried out over all ozone-reactive materials. The air-exchange rate can be estimated from aircraft specifications and basic flight attributes (i.e., ventilation 
rate and pressure). To obtain values for the remaining two parameters, the amount and reactivity of each surface type in the cabin must be evaluated.

Deposition velocity parameterizes uptake of pollutants on surfaces and is formally defined as the flux to a surface divided by the free stream concentration. It is possible to obtain the deposition velocity for a specific material by exposing it to ozone in a chamber void of other ozone-reactive material. At steady state, the deposition velocity is given by equation 3-2 (Morrison and Nazaroff, 2000).

$$
v_{d}=\frac{Q_{\text {chamber }}}{\left[\mathrm{O}_{3}\right]_{\text {chamber }} S_{\text {material }}}\left(\left[\mathrm{O}_{3}\right]_{\text {supply }}-\left[\mathrm{O}_{3}\right]_{\text {chamber }}\right)
$$

where $\left[\mathrm{O}_{3}\right]_{\text {chamber }}$ is the ozone level inside the chamber, $\left[\mathrm{O}_{3}\right]_{\text {supply }}$ is the ozone level in the supply air, $Q_{\text {chamber }}$ is the air-flow rate through the chamber (units of volume per time), and $S_{\text {material }}$ is the nominal surface area of the material. Deposition velocity is a situationspecific parameter in that it may depend on the flow conditions under which the measurements were made. Efforts to describe this dependence have yielded a simplified two-resistor model of ozone uptake (Cano-Ruiz et al., 1993). In this model, the core of an interior space is considered to be well mixed and separated from each ozone-reactive surface by a thin concentration boundary layer. The analogy of two resistors in series describes the rates of two key, sequential processes controlling surface uptake: transport to the surface through the boundary layer and surface reaction kinetics. The transport resistance, $r_{t}$, is the inverse of the transport-limited deposition velocity, $v_{t}$, and the uptake resistance, $r_{s}$, can be expressed as $4 /(\gamma\langle v\rangle)$ so that the deposition velocity is given by equation 3-3. 


$$
\frac{1}{v_{d}}=r_{t}+r_{s}=\left(\frac{1}{v_{t}}+\frac{4}{\gamma\langle v\rangle}\right)
$$

Here, $\langle v\rangle$ is the Boltzmann velocity, which has a value of $3.6 \times 10^{4} \mathrm{~cm} \mathrm{~s}^{-1}$ for ozone at $293 \mathrm{~K}$. The reaction probability, $\gamma$, is defined as the fraction of collisions of ozone molecules at the surface that result in irreversible uptake. By measuring the deposition velocity, $v_{d}$, and the transport-limited deposition velocity, $v_{t}$, to a surface, one can extract the flow-independent parameter, the reaction probability, $\gamma$. The utility of the reaction probability is that it can be combined with information about the flow conditions in the environment of interest, in this case the aircraft cabin, to translate the deposition velocity measured in the laboratory to the expected value for the real environment. Equation 3-4, based on equation 3-3, shows how the reaction probability is calculated given the deposition velocity and mass-transport-limited deposition velocity measured in a chamber. The method for determining a deposition velocity using the reaction probability and flow conditions is given later in equation 3-7, and the process of determining in-situ deposition velocities from $v_{d}$ measured in the chamber is explained in an appendix (Section 3.A).

$$
\gamma=\left(\frac{\langle v\rangle}{4}\left(\frac{1}{v_{d}}-\frac{1}{v_{t}}\right)\right)^{-1}
$$

The transport-limited deposition velocity is obtained experimentally by eliminating uptake resistance at the surface. In practice this is achieved by coating the material with potassium iodide $(\mathrm{KI})$, a substance that is considered to be a perfect sink for ozone (Parmar and Grosjean, 1990). In the research reported here, experiments identical to ones 
conducted to measure $v_{d}$ were also conducted with the material coated in KI to measure $v_{t}$.

In addition to determining how much ozone was consumed in reactions with surfaces, the types and amounts of gas-phase byproducts formed from ozone-initiated reactions were measured. Molar yield of volatile byproducts, $Y_{i}$, defined as moles of species $i$ formed per mole of ozone consumed, was calculated using equation 3-5.

$$
Y_{i}=\frac{\Delta C_{i}}{\left(\left[\mathrm{O}_{3}\right]_{\text {supply }}-\left[\mathrm{O}_{3}\right]_{\text {chamber }}\right)}
$$

where $\Delta C_{i}$ is the increase in the gas-phase abundance of species $i(\mathrm{ppb})$ in the presence of ozone. Emission fluxes with and without ozone were calculated using equation 3-6.

$$
E_{i}=\frac{C_{i} \times Q_{\text {chamber }}}{S_{\text {material }}}
$$

where $C_{i}$ is the measured gas-phase concentration of species $i$ in $\mu \mathrm{mol} \mathrm{m}^{-3}$, and $E$ has units of $\mu \mathrm{mol} \mathrm{m} \mathrm{m}^{-2} \mathrm{~h}^{-1}$.

\subsubsection{Materials}

An inventory of the typical types and amounts of surface materials was determined for a few representative aircraft cabins by personal correspondence with employees of Boeing Aircraft and by consulting scale diagrams of aircraft at www.boeing.com. Details about surface areas and other relevant airplane characteristics are presented in an appendix (Section 3.B.). New and used samples of the most prevalent cabin surfaces (carpet, plastic, and seat fabric) were obtained from the manufacturers via Boeing. Many types of plastic are used in the cabin, and in this study four new manufacturers' samples were investigated: two samples of plastic-coated wall covering 
and two types of plastic used in passenger service units (overhead panels housing passenger lights and gaspers). One used plastic material was also tested, a folding tray table that had been in service for an unknown period of time. Eight samples of new carpet and two used carpets were also studied. One of the used carpets was a runner that had been in service for approximately 18 months since cleaning (total duration in service was unknown). The other used carpet, visibly worn and dirty, had an unknown service life and was stored approximately two years prior to testing. A swatch of new seat fabric and a used seat covering that had been in service for 18 months since its last cleaning were also tested.

Common clothing fabrics (cotton, wool, and polyester) were also included in the study because, under high occupancy, the amount of surface area associated with passenger clothing is substantial relative to the amount of surface area associated with "fixed" cabin surfaces. Also, clothing may contain skin oils, which are reactive with ozone (Fruekilde et al., 1998; Tamás et al., 2006). Previous studies have suggested that worn clothing is a reasonable surrogate for exploring passenger reactivity in the cabin (Tamás et al., 2006; Wisthaler et al., 2005). Both laundered and soiled samples were investigated to evaluate the influence of skin oil on ozone consumption and byproduct formation. Laundered cloth samples were washed in a fragrance- and dye-free detergent and then stored in foil and sealed in a plastic bag and were handled only with gloved hands. Soiled cloth samples were laundered materials that were worn next to the skin by a male, age 25 , while sleeping for $\sim 8 \mathrm{~h}$ just prior to testing. Since only one subject was asked to wear samples, variations in skin oil loading and composition were not 
characterized. Ozone reactions with human skin or hair and with aircraft ventilation ductwork were not investigated.

\subsubsection{Experimental apparatus}

Experiments were conducted in a $10.5 \mathrm{~L}$ electropolished stainless-steel chamber housed in an incubating enclosure, as described in Morrison et al. (1998). The chamber temperature was maintained at $23 \pm 1{ }^{\circ} \mathrm{C}$. "Zero" grade air was humidified by means of running a portion of the air stream through a sparger. Relative humidity $(\mathrm{RH})$ was maintained at $10 \pm 1 \%$. Temperature and RH were measured every minute inside the chamber with a probe (Model HMD30YB; Vaisala). Experiments were performed at standard pressure (1 atm). Ozone was generated by means of UV irradiation of a $0.3 \mathrm{~L}$

$\min ^{-1}$ air stream, which made up part of a total flow of 3-4 $\mathrm{L} \mathrm{min}^{-1}$. Ozone in chamber air was continuously monitored with a photometric ozone analyzer (Model 400E; Advanced Pollution Instrumentation, Inc.). All material samples placed in the chamber were encased in or laid on a foil backing to isolate exposure to one primary surface. Gas samples were collected at the chamber exhaust. The experimental apparatus is shown in Figure 3-2.

\subsubsection{Chemical sampling and analysis}

Volatile organic compound (VOC) samples were collected on TenaxTA-filled tubes (P/N CP-16251; Varian, Inc.) and analyzed by thermal desorption-gas chromatography-mass spectrometry (TD-GC-MS) using a thermal desorber and cryogenic trap (Model CP-4020 TCT; Varian, Inc.) and an HP6890 GC interfaced to a HP5973 mass selective detector. Carbonyl samples were collected on 
dinitrophenylhydrazine (DNPH) coated silica cartridges (P/N WAT037500; Waters Corp.), extracted with acetonitrile, and analyzed for formaldehyde, acetaldehyde, and acetone by high-performance liquid chromatography (Model 1200; Agilent). Details of the analytical methods are reported by Destaillats et al. (2006a). All gas samples were collected and analyzed in duplicate, and background samples were run periodically.

Inline ozone scrubbers were used for VOC collection to avoid ozone-sampling artifacts (Fick et al., 2001; Calogirou et al., 1996). Some of the first experiments in this series were conducted with a commercial, KI-filled scrubber (P/N WAT054420; Waters Corp.) attached upstream of the Tenax and DNPH samplers. The commercial scrubber introduced many unwanted compounds onto the Tenax. Although scrubber artifacts appeared not to interfere with analysis of target analytes $\left(\mathrm{C}_{4}-\mathrm{C}_{10}\right.$ saturated aldehydes, $\mathrm{C}_{7-}$ $\mathrm{C}_{9}$ unsaturated aldehydes, and 6-methyl-5-hepten-2-one), to reduce ambiguity in interpreting the analytical results I developed an in-house scrubber for use with the Tenax samplers. The new scrubber comprised a glass tube with KI held in place with glass wool plugs. The glass tube was the same type used for Tenax samplers, approximately $18 \mathrm{~cm}$ in length with $0.6 \mathrm{~cm}$ outer diameter. The KI was from a commercial scrubber, ground with mortar and pestle; the KI from one commercial scrubber was used to fill two inhouse scrubbers. The in-house scrubber was tested in a side-by-side comparison with the commercial scrubber using five representative compounds: hexanal, heptanal, nonanal, t2-nonenal, and 6-methyl-5-hepten-2-one. The in-house scrubber was highly effective at scrubbing ozone and did not result in significant positive or negative artifacts of the target compounds (with or without ozone) or the appearance of many unwanted compounds in the chromatograms. 


\subsection{Ozone deposition experiments}

\subsubsection{Experimental protocol}

The first set of experiments was performed to screen all of the materials obtained from the aircraft manufacturers and all of the clothing fabrics for ozone reactivity. In screening experiments, the chamber was first quenched by introducing a high ozone concentration in the supply air $(\sim 350 \mathrm{ppb})$ until the concentration at the exhaust equaled the concentration in the supply. Then, the chamber was ventilated with clean air for 15 minutes, after which the material specimen was introduced and exposed to ozone for a period of $3 \mathrm{~h}$ or more. The 3 -h minimum exposure duration was chosen in consideration of cabin ozone regulations (NRC, 2002). The specimen was left in the chamber until the ozone level was approximately steady, i.e. changing by less than 2 ppb per 10 minutes. Flow rate and sample size were adjusted according to the flow-to-surface ratio of the material in a typical aircraft. For materials other than carpet, specimens were approximately $250 \mathrm{~cm}^{2}$ and were exposed to $120 \pm 5 \mathrm{ppb}$ ozone (supply level) at a chamber airflow rate of $4.0 \pm 0.1 \mathrm{~L} \mathrm{~min}^{-1}$. Owing to a limited supply of materials, carpet specimens were $70 \mathrm{~cm}^{2}$ and were exposed to $105 \pm 5 \mathrm{ppb}$ ozone (supply level) at an airflow of $3.0 \pm 1 \mathrm{~L} \mathrm{~min}^{-1}$.

The 3-h average deposition velocity was calculated with equation 3-2 where $\left[\mathrm{O}_{3}\right]_{\text {chamber }}$ was the average ozone level collected from 15 minutes to 195 minutes after sample introduction. The first 15 minutes of data were discarded to allow for stabilization of conditions after opening the chamber. The uncertainty associated with deposition velocities was determined from error propagation analysis to be $<5-10 \%$. Variability 
among specimens was expected to be the larger source of error and was determined to be $<15 \%$ from duplicate and triplicate experiments of a subset of materials.

\subsubsection{Results}

Deposition velocities for the 22 materials in the screening experiments are shown in Table 3-1. The initial, final, and 3-h average deposition velocities ( $v_{d, 15 \min }, v_{d, 195 \min }$, and $v_{d, a v}$, respectively) are given because material reactivity is observed to decrease with time of exposure (see Section 3.4). The 3-h average deposition velocities for all materials range from 0.06 to $0.54 \mathrm{~cm} \mathrm{~s}^{-1}$. The 3-h average reaction probability, $\gamma_{a v}$, values were calculated for each material according to equation 3-4 using the mass-transfer-limited deposition velocity indicated in Table 3-1. A limited number of KI-coated materials was tested because mass-transport-limited deposition velocities were found to be relatively uniform within a surface-material category. A limitation of the KI-coating method should be noted: KI tends to recrystallize when dried on a smooth surface causing uneven coverage and creating a new surface microstructure that could conceivably affect mass transport.

Table 3-1 illustrates several points. First, deposition velocities for all materials were contained within an order of magnitude. Second, the carpet samples (all different carpets) exhibited similar deposition rates except for new carpet 5, which had an unusually high deposition velocity. From BET analysis, this carpet did not possess a significantly higher area than the other carpet samples so the increased reactivity is likely due to a difference in fiber surface treatment or backing (Morrison and Nazaroff, 2002a). Third, used samples were slightly more reactive than new ones in the case of carpet and 
seat fabric. It is possible that accretion of an organic film caused the increase in reactivity (Liu et al., 2003). Fourth, soiled clothing samples were more reactive than freshly laundered samples. Figure 3-3 shows the deposition velocities for the laundered, soiled, and KI-coated clothing fabrics. Wearing the fabric increased its reactivity to near the transport-limited (KI-coated) level. Reaction with skin oil that was transferred to the fabric is very likely responsible for the increase. Skin oil and ozone reactions are discussed in Section 3.4.3.

The deposition velocities measured in this study are higher than values reported in other studies of similar materials (Cano-Ruiz et al., 1993; Morrison and Nazaroff, 2000). Likely, these higher values are a result of the much higher near-surface air flows associated with the higher air-exchange used rate to simulate the cabin environment. Figure 3-4 compares the data collected in this study to a model of deposition velocity as a function of reaction probability and friction velocity (Cano-Ruiz et al., 1993; Morrison and Nazaroff, 2002b). The model is described by equation 3-7:

$$
v_{d}=\frac{\gamma\langle v\rangle u^{*}}{\Gamma \gamma\langle v\rangle+4 u^{*}}
$$

where $u^{*}$ is the friction velocity and $\Gamma$ is a parameter equal to 13.3 for the conditions in this chamber (Morrison and Nazaroff, 2002b). A least-squares fit of the model to the data indicates a friction velocity of $7 \mathrm{~cm} \mathrm{~s}^{-1}$ for the chamber. An estimate of friction velocity in the cabin environment was calculated by inverting equation 3-7 and using values for deposition velocity that were measured a simulated cabin study (Tamás et al., 2006) and reaction probabilities from the current study. The estimated range for $u^{*}$ was $3-5 \mathrm{~cm} \mathrm{~s}^{-1}$ for conditions in the simulated cabin. See the appendix (Section 3.A). for additional 
discussion of how deposition velocities in the chamber are extrapolated to real environments.

\subsubsection{Aging and regeneration in screening experiments}

Decreasing reactivity of a material with increasing cumulative ozone exposure is termed "aging" (Mueller et al., 1973; Sabersky et al., 1973; Morrison and Nazaroff, 2000). Figure 3-5 shows the time-dependent ozone concentration measured at the chamber exhaust for four materials during the screening experiments. Since the ozone level in the supply air and all other experimental conditions were held constant, the residual ozone level in the chamber is an indicator of the reactivity of the material. A high residual ozone concentration indicates low ozone reactivity and vice versa. Some materials appear to have persistent reactivity with ozone on this time scale (i.e. used seat fabric), whereas others have quickly diminishing ozone-reactivity (i.e. new tedlar-coated wall covering). Most materials exhibited time-varying reactivity profiles between these extremes (as illustrated for wool in Figure 3-5).

\subsection{Byproduct emissions experiments}

\subsubsection{Experimental protocol}

A second series of experiments was conducted to characterize primary emissions and ozone-reaction byproducts from cabin materials and clothing. One new and one used specimen of each material (seat fabric, carpet, and plastic) and one laundered and one soiled specimen of each type of the clothing fabric (cotton, polyester, and wool) were tested. Each experiment was run at the same conditions: $10 \pm 1 \% \mathrm{RH}, 23 \pm 1{ }^{\circ} \mathrm{C}, 4.0 \pm$ $0.1 \mathrm{~L} \mathrm{~min}^{-1}, 160 \pm 4 \mathrm{ppb}$ ozone in the supply air. The chamber was first quenched and 
aired as in the screening experiments. The material was then placed in the chamber and conditioned for $3 \mathrm{~h}$ with clean air, after which the ozone was turned on for $1.5 \mathrm{~h}$ or more. During conditioning, duplicate 3-h, $100 \mathrm{~mL} \mathrm{~min}^{-1}$ samples were collected on Tenax-filled glass samplers and 3-h samples were collected on DNPH, one at $0.4 \mathrm{~L} \mathrm{~min}^{-1}$ and the other at $1.0 \mathrm{~L} \mathrm{~min}{ }^{-1}$. Another set of samples was collected during the $1.5 \mathrm{~h}$ period of ozone exposure. In addition to the main experiments, two "background" experiments were conducted: one with the chamber empty and one where the chamber contained only the foil backing. Background levels of the sum of all compounds except acetone were less than the equivalent of $0.25 \mu \mathrm{mol} \mathrm{h}^{-1} \mathrm{~m}^{-2}$ without ozone and $0.5 \mu \mathrm{mol} \mathrm{h}{ }^{-1} \mathrm{~m}^{-2}$ with ozone, and acetone emissions were less than $0.5 \mu \mathrm{mol} \mathrm{h}^{-1} \mathrm{~m}^{-2}$ with or without ozone. The chamber background concentrations were subtracted from measured material emissions. Thus, average emission rates were calculated using equation 3-6 where $C_{i}$ is the concentration of a species $i$ measured during a sampling period minus a background concentration from blank experiments. Across all experiments, the average relative standard deviations (RSD) for formaldehyde, acetaldehyde, acetone, and 6-methyl-5hepten-2-one were $40 \%, 50 \%, 55 \%$, and $30 \%$. For all other compounds the average RSD was less than $15 \%$.

\subsubsection{Emissions from cabin materials}

The techniques used to collect emissions were capable of detecting a wide range of VOCs. Saturated aldehydes $\left(\mathrm{C}_{1}\right.$ through $\left.\mathrm{C}_{10}\right)$, acetone, and 6-methyl-5-hepten-2-one were the compounds most commonly detected. Based on previous studies, unsaturated aldehydes such as 2-nonenal were also likely emitted but at levels too low to be detected 
in the current experiments, and formic and acetic acids were likely produced but not detectable with the collection methods employed (Morrison and Nazaroff, 2002a; Wisthaler et al., 2005). Emissions were generally higher in the presence of ozone. Presented in Figure 3-6 are emission rates from cabin materials and clothing fabrics with and without ozone, and the corresponding yields are presented in an appendix in Table 3C-2. Although all experiments, unless otherwise indicated, were conducted with the same level of ozone in the supply air, the ozone level that a specimen was effectively exposed to varied with the reactivity of the specimen. Average chamber ozone levels are indicated in the emissions figures.

New and used plastic emitted acetone at rate of $\sim 0.5 \mu \mathrm{mol} \mathrm{h}^{-1} \mathrm{~m}^{-2}$ with and without ozone, but emissions of aldehydes were higher in the presence of ozone. Emissions from new plastics were low overall. The secondary emissions profiles of used plastic and used seat fabric were similar, perhaps owing to an accretion of an ozonereactive organic film during service (Liu et al., 2003). New and used seat fabric had similar primary emissions consisting mainly of formaldehyde and acetone. New seat fabric was the highest emitting cabin material, with total secondary emissions comparable to soiled clothing fabrics; ozone reaction byproducts were dominated by acetone and nonanal.

The dominant species found in secondary emissions from used carpet were acetaldehyde and nonanal. High emissions of acetaldehyde were unique to this material, but uncertainty associated with the acetaldehyde measurement in this experimental run was high, with $\sim 100 \%$ RSD. The secondary emissions profile of new carpet was similar to that of laundered clothing fabrics. The unsaturated fatty acids believed to be the 
precursors of these compounds are naturally present in cotton (Pollock, 1948) and may also be present in carpet owing to the use of plant-derived soaps and oils in carpet fabrication and processing (Morrison and Nazaroff, 2002a). The emissions profiles from the carpet samples are comparable to measurements made in similar studies, but are near the lower end of the ranges of reported values (Morrison and Nazaroff, 2002a; Wang and Morrison, 2006).

\subsubsection{Emissions from clean and worn fabric: skin oil chemistry}

Without ozone the VOC emissions from fabrics were very low, whether soiled or laundered. However, when ozone was present, the total VOC emission rates from laundered fabrics were comparable to the cabin materials, and emissions from soiled fabrics were two to three times higher than cabin or laundered fabrics (Figure 3-6b). Two findings make it clear that skin oil transferred onto fabrics during wear was responsible for the increase in reactivity and byproducts from these samples. First, secondary emissions profiles were similar amongst the soiled fabrics. Second, expected byproducts of ozone reactions with squalene dominated secondary emissions from worn fabrics.

Squalene is a triterpene that constitutes $\sim 5-15 \%$ of skin oil (Greene et al., 1970 ; Nicolaides, 1974); it has six unsaturated carbon bonds, which makes it highly reactive with ozone and prone to byproduct formation (Fruekilde et al., 1998). Figure 3-7, adapted from Fruekilde et al. (1998) and Wells et al. (2008), shows the structure of squalene and some its detected reaction byproducts.

The six double bonds of squalene are symmetrical, thus three unique reactions are possible (see Figure 3-7). The initial reaction of ozone with squalene produces a pair of 
squalene degradation products, one lower molecular weight, volatile product, and one higher-molecular weight, semivolatile product that likely stays sorbed to the surface. The lower molecular weight oxidation products of squalene are acetone, 6-methyl-5-hepten-2one (6-MHO), and geranyl acetone (GA), respectively from the first, second, and third double bonds, as numbered in Figure 3-7. Acetone, 6-MHO, and GA are possible primary reaction byproducts of the ozone-squalene reaction; these compounds can also be formed from secondary reactions, i.e. ozone reaction with the second double bond in GA could produce 6-MHO (not shown in Figure 3-7). The semivolatile aldehydes corresponding to the reaction at the second and third double bond of squalene have been tentatively identified in the surface phase (Fruekilde et al., 1998; Wells et al., 2008).

The levels of acetone formed from ozone-initiated skin oil chemistry are not known to cause adverse health effects. The Occupational Safety \& Health Administration Permissible Exposure Limit (OSHA PEL) is 1000 ppm (http://www.atsdr.cdc.gov /tfacts21.html). For comparison, acetone levels measured in an occupied simulated cabin were $20-40 \mathrm{ppb}$ with $\sim 70 \mathrm{ppb}$ ozone present (Weschler et al., 2007). The health effects of 6-MHO and GA are not known at this time. Geranyl acetone (CAS 689-67-8), which is reported to have a floral scent, is used in the chemical fragrance industry; there are no existing health standards, but available Material Safety Data Sheets (MSDS) list GA as an irritant to the eyes, skin, and respiratory system (http://www.thegoodscents company.com/data /rw1014691.html). 6-Methyl-5-hepten-2-one (CAS 110-93-0) has a citrus scent and is also used in the fragrance industry. As with GA, there are no existing health standards for 6-MHO but available MSDSs indicate that in its pure form, it can 
cause eye and respiratory irritation (http://www.thegoodscentscompany.com /msds/md102100.html).

Another byproduct of ozone-squalene chemistry, 4-oxopentanal (4-OPA), is exclusively a secondary byproduct, which is formed by oxidation of 6-MHO and potentially from the other higher molecular weight squalene byproducts such as GA (Smith et al., 1996; Fruekilde et al., 1998, Wells et al., 2008). Low molecular weight organic compounds with multiple oxygenated functional groups, such as 4-OPA, have been found to be sensitizers and irritants using structure activity models and in-vivo assays (Anderson et al., 2007) and also to be associated with the risk of occupational asthma using regression modeling (Jarvis et al., 2005). The formation of acetone and 4OPA from ozone and $\mathrm{OH}$ reacting with 6-MHO has been measured (Smith et al., 1996).

The hydroxyl radical $(\mathrm{OH})$ is commonly formed from ozone-alkene reactions, and $\mathrm{OH}$ may also react with squalene and its reaction byproducts (Atkinson and Arey, 2003). $\mathrm{OH}$ formation from the reaction of ozone and 6-MHO has been confirmed. Based on the chemical structure of squalene and its reaction products, secondary byproducts other than acetone, 6-MHO, GA, and 4-OPA are possible although they have yet to be measured.

Shown in Figure 3-6 are the emissions from soiled and laundered clothing fabrics with and without ozone tested in this study. Acetone dominates secondary emissions from soiled clothing. Average emissions rates of 6-MHO from soiled cotton, polyester, and wool during the first $1.5 \mathrm{~h}$ of ozone exposure (with comparable residual ozone levels) were $2.1,1.0$, and $0.5 \mu \mathrm{mol} \mathrm{h} \mathrm{h}^{-1}$, respectively. The emission rates of 6-MHO from cabin surfaces (seat fabric, plastic, and carpet) were low in comparison to those from 
soiled fabrics, ranging from below the detection limit to $0.2 \mu \mathrm{mol} \mathrm{h} \mathrm{h}^{-1}$. The GC-MS was not calibrated for 4-OPA or GA analysis at the time of these experiments.

In addition to squalene, other constituents of skin oil include free fatty acids, glycerides, wax esters, cholesterol esters, and ceramides (Nicolaides, 1974; Pandrangi and Morrison, 2008). The proportional composition of skin oil is shown in Figure 3-8, and the dominant fatty acid constituents of skin oil are shown in Figure 3-9. The byproducts of these compounds are predominantly decanal and nonanal (Pandrangi and Morrison, 2008). However, there was not a significant difference in the formation of nonanal and decanal between laundered and soiled samples. The other constituents of skin oil may not be as readily transferred to fabric as squalene.

\subsubsection{Yield}

All of the byproducts shown in Figure 3-6 $\left(\mathrm{C}_{1}-\mathrm{C}_{10}\right.$ saturated aldehydes, acetone, and 6-MHO) were included in the total yield calculation. Total molar yield, averaged for each category, is shown in Figure 3-10. Yields by material and by product are shown in an appendix in Tables 3C-1 and 3C-2. Surface category averaged yields ranged from 0.07 to 0.24 . The total aldehyde yield for used carpet (0.08) was similar to the yield (0.07) reported for a 10-y old carpet in another study (Wang and Morrison, 2006).

\subsubsection{Effect of environmental factors}

To explore the effects of various environmental factors on ozone consumption and byproduct formation, supplementary experiments were conducted in which one experimental factor at a time was changed relative to base case conditions. Figure 3-11 
shows emissions from laundered cotton for different ozone supply and RH levels, and the corresponding yields are presented in an appendix in Table 3C-1.

Emission rates of ozone reaction byproducts from cotton, except for formaldehyde, were relatively constant across the different ozone levels tested (inlet levels of $90 \mathrm{ppb}, 160 \mathrm{ppb}$, and $320 \mathrm{ppb}$ ). This may indicate that surface species are the limiting reagents, i.e. ozone is in excess even at the lowest concentration. That formaldehyde emissions increased with increasing ozone concentration while the other species remain constant may suggest that formaldehyde formation, at least in part, results from ozone reactions with cellulose, which is not a limiting reagent, while other byproducts are formed from ozone reactions with oils or other trace compounds present in the fabric (Pollock, 1948).

Significantly more formaldehyde was emitted from the cotton (without ozone present) at $50 \% \mathrm{RH}$ than at $10 \% \mathrm{RH}$. Formaldehyde can be used for "permanent press" treatment to fabrics, and off-gassing can cause elevated formaldehyde levels in indoor settings (Kelly et al., 1999). It is not known whether the cotton obtained for these experiments was treated in this way and the samples used here were washed approximately three times before testing. It is feasible that higher relative humidity improves conditions for formaldehyde release.

Exposing cotton to ozone at $50 \%$ rather than $10 \% \mathrm{RH}$ resulted in an increase in the yield of all byproducts. Emissions of most species increased in proportion to their emissions in the $10 \%$ RH case, approximately doubling for the 50\% RH case. Exceptions are nonanal and decanal, which increased by five times in the 50\% RH case. Increased ozone reactions at the surface of cotton at higher RH have been reported (Destaillats et 
al., 2006b). This result is relevant for the cabin environment because clothing fabric is likely to experience a RH condition higher than the bulk cabin air because of close proximity to human skin.

\subsubsection{Aging and regeneration in emissions experiments}

Some emissions experiments were extended to collect additional integrated samples during ozone exposure. In extended experiments duplicate samples were collected for $3 \mathrm{~h}$ while conditioning the material and then during the first $1.5 \mathrm{~h}$ and the subsequent 2-3 h of ozone exposure. Extended experiments were performed for used carpet, new seat fabric, used seat fabric, soiled cotton, and laundered cotton (4 conditions). Average emissions for all materials except used seat fabric were less in the later sampling period, ranging from 20 to $70 \%$ of emissions in the earlier period; used seat fabric emissions were $\sim 20 \%$ higher in the second ozone-exposure period relative to the first.

Exposed materials can exhibit regeneration, a rebound in reactivity after a period of exposure to ozone-free air (Mueller et al., 1973; Sabersky et al., 1973; Morrison and Nazaroff, 2000). To evaluate regeneration, used seat fabric was exposed to conditions intended to simulate routine plane operations. Following an extended emissions experiment ( $3 \mathrm{~h}$ with clean air, then ozone for $3.5 \mathrm{~h}$ ), the used seat fabric specimen was stored in airtight packaging overnight. The next day the specimen was treated as follows: 3-h conditioning, 1.5-h ozone exposure, 1.5-h conditioning, and 1.5-h ozone exposure. The ozone concentration in the supply air was $155-160 \mathrm{ppb}$. This exposure scheme simulates the environment in a plane that has a 7-h flight segment, is grounded overnight, 
and then flies an 8-h segment in which ozone is encountered intermittently. The initial (15 minutes after ozone was turned on) and 90-minute deposition velocities for the three periods were, in sequential order, 0.389 and $0.354 \mathrm{~cm} \mathrm{~s}^{-1}, 0.371$ and $0.326 \mathrm{~cm} \mathrm{~s}^{-1}$, and 0.315 and $0.295 \mathrm{~cm} \mathrm{~s}^{-1}$ (see Table 3-2). Thus, the material exhibited aging: the deposition velocities were lower at the end of each period than at the beginning, and each period had successively lower deposition velocities. The material also appears to exhibit regeneration from the first to second ozone exposure period $\left(0.354\right.$ to $\left.0.371 \mathrm{~cm} \mathrm{~s}^{-1}\right)$ but the increase in reactivity is not statistically significant. Although the average reactivity of the material decreased with each exposure to ozone, the total emissions of $C_{1}$ through $C_{10}$ aldehydes increased slightly in sequential ozone exposures with 1.5 -h average emissions of 2.4, 2.7, and $3.1 \mu \mathrm{mol} \mathrm{m} \mathrm{m}^{-2}$, respectively, for the three ozone periods. Emissions during the second and third conditioning periods were low, 0.5 and $0.8, \mu \mathrm{mol} \mathrm{m} \mathrm{m}^{-2} \mathrm{~h}^{-1}$, respectively, indicating that residual emissions of desorbing byproducts made no more than a small contribution to total measured emissions.

\subsection{Contributions of surface ozone reactivity to cabin air quality}

The relative abundance of each material in a cabin environment was estimated based on the interior dimensions of a Boeing 737 as an example. The relative amount of each type of surface does not vary much amongst plane types. The surface-to-volume ratios for several planes are given in an appendix (Section 3.B.). To estimate the contribution of passengers to surface area, the cabin was assumed to be fully occupied and half of the seat fabric was assumed to be covered by passengers. Each passenger was 
assumed to contribute $1 \mathrm{~m}^{2}$ of exposed clothing fabric. As shown in Figure 3-12a, plastic and clothing are the dominant contributors to cabin surface area for these conditions.

Although the deposition velocities in the cabin may differ from those measured in chamber experiments, experimental values reported here can be used to indicate relative reactivity. The product of the material-averaged deposition velocity and the surface area to volume ratio of that material in the plane $\left(v_{d} \times S / V\right)$ provides an estimate of the contribution of each surface type to ozone consumption in the cabin. As shown in Figure 3-12b, clothing and seat fabric are the dominant ozone-consuming surfaces.

Since molar yield expresses the amount of product formed per amount of ozone consumed, multiplying yield by ozone deposition velocity provides a parameter that is proportional to byproduct emission rate. Therefore, an estimate of each material's contribution to byproduct emissions into the cabin can be obtained as the product of three terms: average molar yield for a surface, the average deposition velocity to that surface, and the amount of that surface in the cabin $\left(Y \times v_{d} \times S / V\right)$. The total yield of $\mathrm{C}_{1}$ through $\mathrm{C}_{10}$ saturated aldehydes, acetone, and 6-MHO, averaged for each material type, was used to produce Figure 3-12c. This analysis suggests that clothing fabric dominates ozoneinitiated byproduct emissions, followed by seat fabric, although all material types make significant contributions. These results are substantiated by relatively good agreement with results from a simulated cabin study (Tamás et al., 2006).

\subsection{Byproduct exposure in the cabin}

In this section, intake rates of ozone reaction byproducts are estimated for the aircraft cabin and compared to other environments. To accomplish this, a relatively 
simple model is employed to predict byproduct concentrations in the cabin environment. Data from this study and from other recent studies of cabin air quality provide the necessary input for the model.

\subsubsection{Predicting byproduct levels}

Byproduct levels can be estimated using three key parameters: cabin ozone concentration $\left(\left[\mathrm{O}_{3}\right]_{\text {cabiin }}\right)$, retention ratio $(R)$, and byproduct yield $(Y)$, according to equation 3-8 (adapted from NRC, 2002).

$$
C_{i}=Y_{i}\left(\frac{1}{R}-1\right)\left[\mathrm{O}_{3}\right]_{c a b i n}
$$

Here $\mathrm{C}_{i}$ is the cabin level of species $i$ attributable to byproduct formation (ppb). This model is derived by assuming the cabin is a well-mixed environment, which is a reasonable assumption when considering ozone consumption. In modern commercial aircraft, air flows into the cabin just above the luggage storage bins and mixes in a circular pattern before being removed at the base of the plane wall (see Figure 3-13). This flow pattern results in good mixing in the radial direction (i.e. each section of the plane is well-mixed), even though air tends not to mix in the axial direction (i.e. various sections of the plane may experience different conditions) (Mazumdar and Chen, 2008). However, for ozone, axial uniformity will be promoted for two reasons. First, the supply air is the source of ozone and it discharges throughout the cabin. Second, approximately half of cabin air is recycled. In the following paragraphs, ranges of the three key parameters are discussed. 
Real-time cabin ozone data were recently collected on 68 U.S.-based domestic flights segments with a minimum duration of $3.5 \mathrm{~h}$. For the 46 flights without ozone converters, flight-averaged ozone ranged from 5 to $140 \mathrm{ppb}$ with an arithmetic mean of $28 \mathrm{ppb}$. Peak-hour ozone (highest rolling 1-h average of ozone data for each flight) ranged from 7 to $250 \mathrm{ppb}$ with a mean of $47 \mathrm{ppb}$. This study indicates that, for flights without ozone control devices, the ozone levels in the cabin can range from tens to lowhundreds of ppb. Data from the 46 flight segments were lognormally distributed; the peak-hour ozone had a geometric mean (GM) of $33 \mathrm{ppb}$ and a geometric standard deviation (GSD) of 2.3, and the flight-averaged ozone level was $20 \mathrm{ppb}$ and 2.1 (GM, GSD). Strictly speaking, what is referred to here as "flight-averaged" data is a "sample" average where the "sample" does not include take-off and landing.

The retention ratio can be estimated using equation 3-1; the important parameters are air-exchange rate, surface-to-volume ratios, and ozone deposition velocities. Typical air-exchange rates for aircraft cabins are $10-15 \mathrm{~h}^{-1}$ (NRC, 2002). Surface-to-volume ratios of each material are estimated from airplane dimensions (see Section 3.B.). Deposition velocities for each material were measured in this study and then extrapolated to estimate an average value for the cabin. Determining cabin deposition velocities from chamber data requires information about flow conditions in that environment, such as the friction velocity (see Section 3.B.). Cabin airspeeds are comparable to those in buildings, and surface topographies are probably more complex, suggesting somewhat higher friction velocities (Matthews et al., 1989; Zhang and Chen, 2007). Deposition velocities measured in this study were adjusted for a range of friction velocities $\left(3-7 \mathrm{~cm} \mathrm{~s}^{-1}\right)$ using equation 3-7. The resulting range of estimates for the retention ratio was $0.2-0.4$, i.e. 60 - 
$80 \%$ of ozone is estimated to be consumed on surfaces once it enters the cabin. For comparison, a retention ratio of 0.21 was measured in a fully occupied simulated cabin with an air-exchange rate of $8.8 \mathrm{~h}^{-1}$ (Tamás et al., 2006).

In this chapter it was established that $\mathrm{C}_{1}$ through $\mathrm{C}_{10}$ saturated aldehydes, 6-MHO, and acetone were detected from individual cabin surfaces exposed to ozone, and total yields were in the range of 0.07 to 0.24 . Another study, conducted at nearly the same time as this work, exposed 16 human subjects to ozone in a simulated cabin chamber for a simulated flight segment. VOC measurements were made with a PTR-MS, which is capable of detecting a very wide range of compounds in real-time. This study established that byproducts of ozone-initiated chemistry in the cabin include saturated and unsaturated aldehydes (e.g. nonanal, decanal, and nonenal), carboxylic acids (e.g. formic and acetic acid), and squalene oxidation products (e.g. acetone, 6-MHO, 4-OPA, and geranyl acetone) (Weschler, 2007). Overall byproduct yields for that study were estimated to be in the range $0.25-0.3$.

Now that a range of reasonable values has been established for each of the three key parameters, let's examine the form of the model. Lumping the first two terms, $Y_{i}\{(1 / R)-1\}$, on the right hand side of equation 3-8 produces a "byproduct factor" by which the cabin ozone level can be multiplied. The advantage of calculating the byproduct factor is that it illustrates how oxidation byproduct level can be predicted from and compared to the measured cabin ozone level. With $Y$ equal to $0.25-0.3$, and R equal to $0.2-0.4$, the resulting byproduct factor is in the range $0.4-1.2$. That means that the predicted cabin oxidation byproduct level is $0.4 \times$ to $1.2 \times$ the cabin ozone level. The reason that the byproduct level can be higher than the ozone level, even though yield is 
less than one, is that more ozone is consumed by surface reactions than remains in the air. (Note that the term $\{(1 / R)-1\}\left[\mathrm{O}_{3}\right]_{\text {cabin }}$ reflects the amount of ozone consumed by reactions within the cabin.) Therefore, relatively more byproducts than ozone can be present on a molar basis.

Figure 3-14 shows the results of a Monte Carlo simulation applied to equation 38. In this simulation, the input parameters, yield $(Y)$ and retention ratio $(R)$, were represented as having normal distributions with a mean of 0.3 and a standard deviation of 0.05. A slightly higher value for yield was assumed in this simulation than was detected by Weschler et al., (2007) in attempt to account for the "stealth" byproducts of ozoneinitiated chemistry. The ozone level distribution was set using the fitted distribution parameters from Bhangar et al., (2008) of peak-hour ozone data gathered on 46 domestic flights without converters; peak-hour ozone was lognormally distributed with a geometric mean of $33 \mathrm{ppb}$ and geometric standard deviation of 2.3. One million model simulations were run. The resulting distribution of byproduct levels was also lognormal with a geometric mean of $21 \mathrm{ppb}$ and geometric standard deviation of 2.4. To give an indication of the upper range of exposure levels, the $95^{\text {th }}$ percentile for peak-hour byproduct level would be $115 \mathrm{ppb}$. When the simulation was run with the flight-averaged ozone level distribution being lognormal with $\mathrm{GM}=20 \mathrm{ppb}$ and $\mathrm{GSD}=2.1$, the resulting byproduct distribution was lognormal with $\mathrm{GM}=13 \mathrm{ppb}$ and $\mathrm{GSD}=2.2$. The major result of this analysis is that the range of byproduct levels is similar to the range of ozone levels, in the tens to low-hundreds of $\mathrm{ppb}$ for planes that are not equipped with converters.

The total yield reflects the sum of byproducts that have been detected. Byproducts that we are not currently capable of measuring, such as fast-reacting radicals or thermally 
labile compounds (Weschler, 2006), may play an important role in comfort or heath effects and are not included in this assessment. At this time it is unknown how total byproduct level correlates with health and comfort endpoints, but there are associations between the degradation of perceived air quality and the oxidation of surface materials (Weschler, 2006; Wolkoff et al., 2006). Some byproducts are irritating or harmful, and others may not be, but easily detectable compounds serve as surrogates for detection of oxidation byproduct formation.

\subsubsection{Estimating byproduct intake}

It can be difficult to compare exposures to oxidation byproducts amongst different environments (i.e. in the office, at home, on a plane, or outdoors) because most of the common products of ozone-initiated chemistry are not measured in ambient air and measurements in indoor environments do not exist or have not been catalogued as many outdoor air pollutants have. In the following analysis, I chose three oxidation products formaldehyde, nonanal, and 4-oxopentanal - that are characteristic of ozone-initiated reactions in indoor environments. Typical concentrations of these compounds in the aircraft cabin environment are calculated using a model presented in the previous section. Concentrations of these compounds in homes and in the outdoor environment were estimated from the literature. A flight attendant's exposure to these three byproducts in a variety of environments is compared using inhalation intake rate as a metric.

Formaldehyde, nonanal, and 4-OPA concentrations in the airplane were estimated using equation 3-8 with yields of $0.3 \%, 3.6 \%$, and $2.6 \%$, respectively (from Weschler et 
al., 2007), a retention ratio of 0.3 , and a moderate cabin ozone level for a flight without a converter of $30 \mathrm{ppb}$ (Bhangar et al., 2008).

Formaldehyde (HCHO) is a pollutant for which health effects have been established and concentration data has been measured in many environments (CARB, 2004). Formaldehyde is an oxidation product, and it is also directly emitted from wood products and adhesives. An average indoor formaldehyde concentration was estimated from a compilation of indoor monitoring studies (CARB, 2004). An average value of outdoor formaldehyde concentration was based on modeled concentrations from the 1999 National-Scale Air Toxics Assessment (EPA, 1999).

Unlike formaldehyde, 4-oxopentanal (4-OPA) is not widely measured and its health effects are only now being studied. However, this compound is likely to be present in both indoor and outdoor environments whenever ozone is present, because it is a secondary oxidation product of squalene, a component of skin oil and waxy leaf films (Fruekilde et al., 1998). Linalool, a floral scented terpene alcohol, could also be a source of 4-OPA (Shu et al., 1997). One group of researchers has made ambient measurements of 4-OPA near a forest in Japan and found that the concentration ranged from 0.2 to 2.6 $\mu \mathrm{g} \mathrm{m}{ }^{-3}$ (Matsunaga et al., 2004). A lower value of $0.2 \mu \mathrm{g} \mathrm{m}^{-3}$ was assumed for the outdoor concentration because 4-OPA would likely be lower in urban areas with lower vegetation density. Ozone-initiated reactions with skin oil are potentially a much more important source of 4-OPA from a health perspective; in the presence of ozone, byproducts of skin oil are produced in the immediate breathing zone. To date, 4-OPA levels from ozone reactions with human occupants have only been measured in a simulated cabin environment (Weschler et al., 2007). It is unknown how exposure to 
ozone indoors versus outdoors affects intake of skin oil oxidation byproducts; the ozone concentration would be higher outdoors than indoors, but the dilution rate would also be higher.

The indoor level of 4-OPA was estimated by determining the average 4-OPA emission rate per person in the cabin (Weschler et al., 2007) and using that to calculate the 4-OPA concentration in a typical room of $30 \mathrm{~m}^{3}$ with an air-exchange rate of $0.5 \mathrm{~h}^{-1}$ and an ozone concentration $\sim 10 \mathrm{ppb}$. (See Table 3-3 footnotes for details.)

The amount of information about aliphatic aldehydes, which are known for their odors, is between that for formaldehyde and for 4-OPA. Nonanal, for example, is becoming commonly measured in not-yet-frequent studies of indoor ozone chemistry and its odor threshold has been established. Ambient nonanal data are virtually nonexistent. (A search of "nonanal AND (outdoor OR ambient)" in Web of Knowledge returns no relevant studies.) The absence of ambient data presumably is because nonanal, if present outdoors, is present at quite low levels. In the indoor environment, however, nonanal is commonly detected. Two inventories of indoor VOCs identified nonanal as one of the most prevalent compounds: one of U.S. office buildings (Girman et al., 1999) and one of Swedish housing stock (Bornehag and Stridh, 2000). A mid-range value from the U.S. study of $5 \mu \mathrm{g} \mathrm{m}^{-3}$ was used in this analysis.

Table 3-3 shows intake rates of formaldehyde ( $\mathrm{HCHO})$, nonanal, and 4oxopentanal (4-OPA) as estimated for a flight attendant. Intake rate, i.e. pollutant mass inhalation rate per person, is determined by multiplying breathing rate $\left(\mathrm{m}^{3} / \mathrm{h}\right)$ by pollutant concentration $\left(\mu \mathrm{g} / \mathrm{m}^{3}\right)$ (Nazaroff, 2008). In this case, intake rate was also multiplied by the duration (hours per week) spent in each type of environment, so that cumulative 
exposure could be compared by environment considering week-long periods. It was assumed that a flight attendant might spend $30 \mathrm{~h}$ of his or her week in flight, $15 \mathrm{~h}$ outdoors (keeping with the national average of about $10 \%$ of time spent outdoors (Klepeis et al., 2001), and the remaining time, $123 \mathrm{~h}$, indoors at home. The breathing rates in the cabin and outdoors are greater than the indoor breathing rate because the flight attendant would be expected to be moving more vigorously in these environments.

For the case of the flight attendant, estimated intakes of nonanal and 4-OPA are similar in both indoor environments, despite spending much more time at home than in the aircraft cabin. The higher concentrations of byproducts in the cabin result from the higher surface-to-volume ratio, occupant density, and ozone level. The table also shows that the dominant exposure to formaldehyde would not occur on a plane, but more likely in the home owing to the much higher concentrations from primary emissions and greater time spent in that environment.

Clearly, total intake of cabin oxidation products would decrease with decreasing time spent in the cabin, thus a typical business traveler would only experience a fraction of the flight attendant's intake, and the cabin may not be an important source of exposure for the casual traveler. However, from a cumulative exposure standpoint, the cabin is an environment of concern only for those who spent a significant amount of time there, including crew members and frequent flyers. From the perspective of acute health and comfort concerns, all crew and passengers may benefit from the addition of ozone converters, and thus the minimization of exposure to odorous and irritating oxidation byproducts. 


\subsubsection{Pressure effects}

Although the experiments reported in this chapter were conducted at a pressure of $1 \mathrm{~atm}$, rather than at the reduced pressure of the cabin environment, we expect ozone deposition velocities and byproduct yields to be relatively independent of cabin pressure. Byproduct emission fluxes from cabin materials likely scale with the ozone partial pressure, so that the results reported here would be scaled down by the ratio of the cabin air pressure to sea-level air pressure in translating to the cabin environment.

\subsection{Conclusions}

The aircraft cabin is different from other indoor environments because of the low relative humidity, high air-exchange rate, high occupant density, high surface-to-volume ratio, low cabin pressure, and the potential to experience high ozone levels. As in other indoor environments, ozone reactions with surfaces lower the ozone levels but produce secondary volatile products including compounds that are odorous, cause sensory irritation, or are toxic. In this study, ozone uptake and byproduct formation of individual materials were characterized through chamber experiments conducted at flight-relevant conditions. Consistently, VOC levels were higher with ozone than without; measured byproducts included formaldehyde, acetaldehyde, $\mathrm{C}_{3}$ through $\mathrm{C}_{10}$ saturated aldehydes, and the squalene oxidation products, acetone and 6-MHO. Due to limitations of available methods, only products that are chemically stable can be captured and analyzed using methods employed here. Other, so-called "stealth" products are known to be formed from ozone-initiated chemistry, including radicals and labile organics (Weschler, 2006).

Deposition velocities and molar yields measured from the experiments were used together with the typical amounts of surfaces in the cabin to estimate the relative 
contributions of surfaces to ozone consumption and emissions. The majority of both could be attributed to seat fabric and clothing, particularly soiled clothing. An important implication of these results is that ozone reactions are occurring on or very near the crew and passengers, which may be significant for exposure. Another important implication is that occupant density in the cabin would likely influence the levels of both ozone and its reaction byproducts in cabin air.

Emissions profiles measured in the chamber experiments were consistent with those measured in a simulated cabin environment (Wisthaler et al., 2005; Weschler et al., 2007). Certain aspects of surface-ozone chemistry can be explored in chamber experiments with the advantage that materials can be isolated and efficiently tested under well-controlled conditions.

By modeling oxidation byproduct formation in the cabin, I have determined that during flight, total byproduct levels are likely similar to the cabin ozone level on a molar concentration basis. For compounds that are present because of surface oxidation reactions, intake occurs almost exclusively indoors. Inhalation of squalene oxidation products and "stealth" products of ozone reactions will higher in the cabin than in most buildings owing to the high occupant density. The present results advance our understanding of the role of ozone-initiated chemistry in influencing air quality in aircraft cabins and in other occupied environments. 
Table 3-1: Initial, final and 3-h average ozone deposition velocities $\left(v_{d, 15 \mathrm{~min}}, v_{d, 195 \mathrm{~min}}\right.$, and $v_{d, a v}$, respectively) and 3-h average reaction probability $\left(\gamma_{a v}\right)$ for 22 cabin materials. ${ }^{\text {a }}$

\begin{tabular}{|c|c|c|c|c|c|}
\hline $\begin{array}{l}\text { Material } \\
\text { Category }\end{array}$ & Material Description & $\begin{array}{l}v_{d, 15 \min } \\
\left(\mathrm{cm} \mathrm{s}^{-1}\right)\end{array}$ & $\begin{array}{l}v_{d, 195 \min } \\
\left(\mathrm{cm} \mathrm{s}^{-1}\right)\end{array}$ & $\begin{array}{c}v_{d, a v} \\
\left(\mathrm{~cm} \mathrm{~s}^{-1}\right)\end{array}$ & $\gamma_{a v}{ }^{b}$ \\
\hline \multirow{10}{*}{ Carpet } & New Carpet 1 & 0.61 & 0.17 & 0.30 & $6.3 \times 10^{-5}$ \\
\hline & New Carpet 2 & 0.68 & 0.30 & 0.39 & $1.1 \times 10^{-4}$ \\
\hline & New Carpet 3 & 0.87 & 0.11 & 0.30 & $6.4 \times 10^{-5}$ \\
\hline & New Carpet 4 & 0.49 & 0.21 & 0.26 & $4.7 \times 10^{-5}$ \\
\hline & New Carpet 5 & 0.95 & 0.38 & 0.54 & $3.7 \times 10^{-4}$ \\
\hline & New Carpet 6 & 0.68 & 0.14 & 0.30 & $6.1 \times 10^{-5}$ \\
\hline & New Carpet 7 & 0.38 & 0.22 & 0.25 & $4.6 \times 10^{-5}$ \\
\hline & New Carpet 8 & 0.60 & 0.18 & 0.30 & $6.0 \times 10^{-5}$ \\
\hline & Used Carpet 1 & 0.73 & 0.18 & 0.32 & $1.6 \times 10^{-4}$ \\
\hline & Used Carpet 2 & 1.08 & 0.18 & 0.36 & $3.1 \times 10^{-4}$ \\
\hline \multirow{6}{*}{$\begin{array}{l}\text { Clothing } \\
\text { Fabric }\end{array}$} & Laundered Cotton & 0.40 & 0.22 & 0.30 & $8.0 \times 10^{-5}$ \\
\hline & Soiled Cotton & 0.62 & 0.31 & 0.41 & $2.2 \times 10^{-4}$ \\
\hline & Laundered Wool & 0.22 & 0.06 & 0.10 & $1.5 \times 10^{-5}$ \\
\hline & Soiled Wool & 0.45 & 0.28 & 0.37 & $2.1 \times 10^{-4}$ \\
\hline & Laundered Polyester & 0.37 & 0.05 & 0.11 & $1.6 \times 10^{-5}$ \\
\hline & Soiled Polyester & 0.55 & 0.37 & 0.46 & $2.7 \times 10^{-4}$ \\
\hline \multirow{2}{*}{$\begin{array}{l}\text { Seat } \\
\text { fabric }\end{array}$} & New Seat Fabric & 0.37 & 0.37 & 0.38 & $1.5 \times 10^{-4}$ \\
\hline & Used Seat Fabric & 0.36 & 0.36 & 0.37 & $1.4 \times 10^{-4}$ \\
\hline \multirow{4}{*}{ Plastic } & New Wall Covering 1 & 0.77 & 0.02 & 0.13 & $2.2 \times 10^{-5}$ \\
\hline & New Wall Covering 2 & 0.46 & 0.02 & 0.12 & $2.0 \times 10^{-5}$ \\
\hline & New Plastic 1 & 0.89 & 0.02 & 0.18 & $4.2 \times 10^{-5}$ \\
\hline & New Plastic 2 & 0.72 & 0.01 & 0.06 & $8.4 \times 10^{-6}$ \\
\hline
\end{tabular}

${ }^{\mathrm{a}}$ Carpet specimens had an exposed nominal surface area of $70 \mathrm{~cm}^{2}$ and were exposed to $105 \pm 5 \mathrm{ppb}$ ozone (supply level) at $10 \pm 1 \% \mathrm{RH}$ with an air-exchange rate of $17 \mathrm{~h}^{-1}$. All other specimens had an exposed nominal surface area of $250 \mathrm{~cm}^{2}$ and were exposed to $120 \pm 5 \mathrm{ppb}$ ozone (supply level) at $10 \pm 1 \% \mathrm{RH}$ with an airexchange rate of $23 \mathrm{~h}^{-1}$.

$\mathrm{b}$ The reaction probability was calculated using equation 3-4 where the 3-h average deposition velocity was used for $v_{d}$. These values of transport-limited deposition velocity were used to calculate reaction probability for each material type: new carpet, $v_{t}=0.65 \mathrm{~cm} \mathrm{~s}^{-1}$; used carpet, $v_{t}=0.41 \mathrm{~cm} \mathrm{~s}^{-1}$; cotton, $v_{t}=0.52 \mathrm{~cm} \mathrm{~s}^{-1}$; wool, $v_{t}=0.46 \mathrm{~cm} \mathrm{~s}^{-1}$; polyester, $v_{t}=0.57 \mathrm{~cm} \mathrm{~s}^{-1}$; seat fabric, $v_{t}=0.52 \mathrm{~cm} \mathrm{~s}^{-1}$; plastic, $v_{t}=$ $0.34 \mathrm{~cm} \mathrm{~s}^{-1}$. 
Table 3-2: Ozone regeneration experiments with used seat fabric.

\begin{tabular}{|c|c|c|c|c|c|}
\hline & $\begin{array}{l}\text { Ozone } \\
\text { condition }\end{array}$ & $\begin{array}{l}\text { Inlet ozone } \\
\text { level (ppb) }\end{array}$ & $\begin{array}{c}v_{\text {dinitial }} \\
\left(\mathrm{cm} \mathrm{s}^{-1}\right)^{\mathrm{a}}\end{array}$ & $\begin{array}{c}v_{d \text { ffinal }} \\
\left(\mathrm{cm} \mathrm{s}^{-1}\right)^{\mathrm{b}}\end{array}$ & $\begin{array}{c}\text { Emissions } \\
\left(\mu \mathrm{mol} \mathrm{m} \mathrm{m}^{-2} \mathrm{~h}^{-1}\right)^{\mathrm{c}}\end{array}$ \\
\hline \multicolumn{6}{|l|}{ Day 1} \\
\hline $0-3 \mathrm{~h}$ & no ozone & 0 & - & - & $1.2^{\mathrm{d}}$ \\
\hline $3-4.5 \mathrm{~h}$ & ozone & 160 & 0.389 & 0.354 & 2.4 \\
\hline \multicolumn{6}{|l|}{ Day 2} \\
\hline $0-3 \mathrm{~h}$ & no ozone & 0 & - & - & 0.5 \\
\hline $3-4.5 \mathrm{~h}$ & ozone & 155 & 0.371 & 0.326 & 2.7 \\
\hline $4.5-6 \mathrm{~h}$ & no ozone & 0 & - & - & 0.8 \\
\hline $6-7.5 \mathrm{~h}$ & ozone & 155 & 0.315 & 0.295 & 3.1 \\
\hline
\end{tabular}

${ }^{a} v_{\text {d,initial }}$ is the deposition velocity 15 minutes after the ozone was turned on.

${ }^{\mathrm{b}} v_{d, \text { final }}$ is the deposition velocity 90 minutes after the ozone was turned on.

${ }^{c}$ 90-minute averaged emission of $\mathrm{C}_{1}-\mathrm{C}_{10}$ aldehydes.

${ }^{\mathrm{d}}$ Relatively high emissions in this no-ozone period are due to high emissions of formaldehyde, and uncertainty associated with formaldehyde for this period was high. 
Table 3-3: Flight attendant's estimated weekly inhalation intake rate of three ozone byproducts in various environments.

\begin{tabular}{cccccc}
\hline Species & Environment & Concentration & $\begin{array}{c}\text { Breathing } \\
\text { Rate }^{\mathrm{a}}\end{array}$ & Duration & Intake Rate \\
\hline \hline \multirow{2}{*}{$\mathrm{HCHO}$} & Airplane & $0.2 \mu \mathrm{g} / \mathrm{m}^{3 \mathrm{~b}}$ & $0.7 \mathrm{~m}^{3} / \mathrm{h}$ & $30 \mathrm{~h} /$ week & $4 \mu \mathrm{g} /$ week \\
& Outdoor & $1 \mu \mathrm{g} / \mathrm{m}^{3 \mathrm{c}}$ & $0.7 \mathrm{~m}^{3} / \mathrm{h}$ & $15 \mathrm{~h} /$ week & $11 \mu \mathrm{g} /$ week \\
& Home & $10 \mu \mathrm{g} / \mathrm{m}^{3 \mathrm{~d}}$ & $0.5 \mathrm{~m}^{3} / \mathrm{h}$ & $123 \mathrm{~h} /$ week & $615 \mu \mathrm{g} /$ week \\
\hline \multirow{2}{*}{ Nonanal } & Airplane & $13 \mu \mathrm{g} / \mathrm{m}^{3 \mathrm{~b}}$ & $0.7 \mathrm{~m}^{3} / \mathrm{h}$ & $30 \mathrm{~h} /$ week & $252 \mu \mathrm{g} /$ week \\
& Outdoor & $<0.1 \mu \mathrm{g} / \mathrm{m}^{3 \mathrm{e}}$ & $0.7 \mathrm{~m}^{3} / \mathrm{h}$ & $15 \mathrm{~h} /$ week & $<1 \mu \mathrm{g} /$ week \\
& Home & $5 \mu \mathrm{g} / \mathrm{m}^{3 \mathrm{f}}$ & $0.5 \mathrm{~m}^{3} / \mathrm{h}$ & $123 \mathrm{~h} /$ week & $308 \mu \mathrm{g} /$ week \\
\hline \multirow{3}{*}{$4-$ OPA } & Airplane & $6 \mu \mathrm{g} / \mathrm{m}^{3 \mathrm{~b}}$ & $0.7 \mathrm{~m}^{3} / \mathrm{h}$ & $30 \mathrm{~h} /$ week & $126 \mu \mathrm{g} /$ week \\
& Outdoor & $0.2 \mu \mathrm{g} / \mathrm{m}^{3 \mathrm{~g}}$ & $0.7 \mathrm{~m}^{3} / \mathrm{h}$ & $15 \mathrm{~h} /$ week & $2 \mu \mathrm{g} /$ week \\
& Home & $2 \mu \mathrm{g} / \mathrm{m}^{3 \mathrm{~h}}$ & $0.5 \mathrm{~m}^{3} / \mathrm{h}$ & $123 \mathrm{~h} /$ week & $123 \mu \mathrm{g} /$ week \\
\hline
\end{tabular}

${ }^{\mathrm{a}}$ A higher breathing rate was assumed for time spent outdoors and in the plane because these periods likely involve more activity.

${ }^{\mathrm{b}}$ Airplane concentrations determined using equation 3-8 with a cabin ozone level of 30 $\mathrm{ppb}$, a retention ratio of 0.3 , and yields from Weschler et al. (2007) of $0.3 \%$ for formaldehyde. $3.6 \%$ for nonanal, and $2.6 \%$ for 4-OPA. Molar fractions were converted to mass concentrations assuming a pressure of $0.8 \mathrm{~atm}$ (33 moles of air per cubic meter).

c Outdoor formaldehyde level from 1999 National-scale Air Toxics Assessment (EPA, 1999).

${ }^{\mathrm{d}}$ Residential formaldehyde concentration from all indoor sources, not just oxidation reactions, California Air Resources Board (CARB, 2004)

${ }^{\mathrm{e}}$ Nonanal values are not reported in outdoor air owing to low concentrations (see text).

${ }^{\mathrm{f}}$ Mid-range value from Girman et al., 1999.

${ }^{g}$ Outdoor 4-OPA level estimated from Matsunaga et al. (2004) Note that this is an estimate of the "background" level of 4-OPA. A person who was exposed to ozone outdoors would likely also be exposed to ozone byproducts because of reactions with skin oil. The ozone concentration would be higher outdoors than indoors, but the dilution rate would also be higher, so it is unknown how exposure to ozone indoors versus outdoors affects byproduct intake.

${ }^{\mathrm{h}}$ Residential 4-OPA level estimated by assuming a $10 \mathrm{ng} \mathrm{s}^{-1}$ emission rate from one person in a $30 \mathrm{~m}^{3}$ room with an air-exchange rate of $0.5 \mathrm{~h}^{-1}$. The emission rate of 4OPA was calculated to be $50 \mathrm{ng} \mathrm{s}^{-1}$ per person with $\sim 70 \mathrm{ppb}$ cabin ozone level from Weschler et al. (2007). The emissions estimate was lowered to $10 \mathrm{ng} \mathrm{s}^{-1}$ per person for the indoor environment because a lower indoor ozone level is expected (in the low tens of ppb). Again it is emphasized that 4-OPA is formed in the near the breathing zone, so average concentrations may not accurately reflect total intake. 

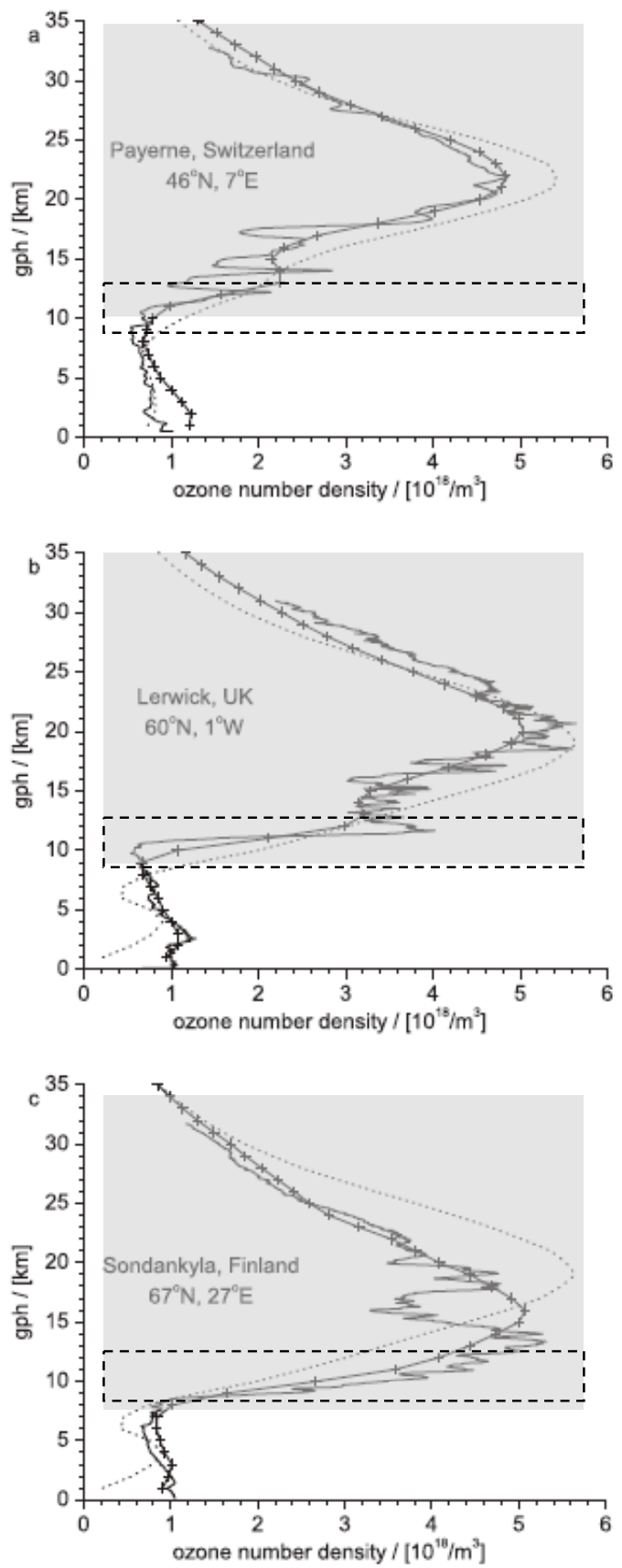

Figure 3-1: Caption on next page. 
Figure 3-1: Examples of atmospheric ozone profiles taken from Müller et al. (2003, Figure 9). Each frame shows three ozone profiles: one measured by ozonesonde (most variable) and two modeled. The ozonesonde profiles were taken in April of 1997 at the location indicated. GPH is geopotential height. Superimposed on each plot is a gray box indicating the ozone layer and a dashed-outline box indicating the typical cruising altitude for commercial aircraft. The plots illustrate the shape of the ozone layer in the atmosphere, and indicate that tropopause height decreases with increasing latitude, and thus the likelihood of encountering elevated ozone levels while flying increases with increasing latitude and altitude. 


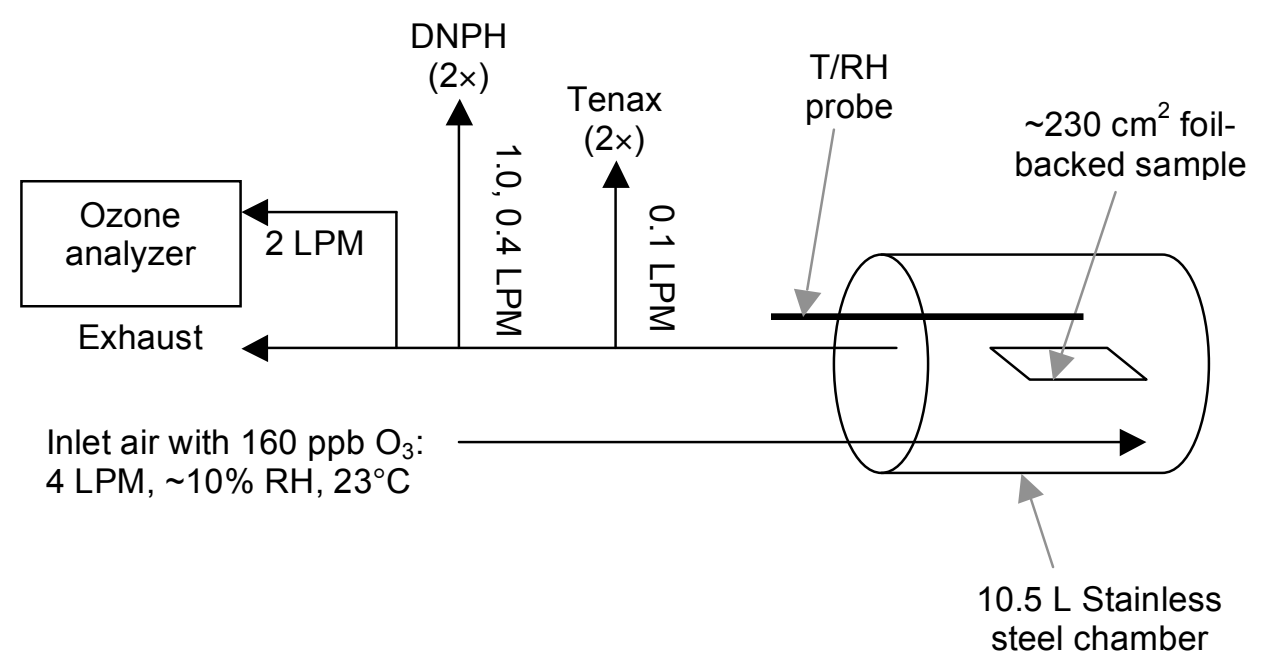

Figure 3-2: Schematic diagram of experimental apparatus for emissions experiments.

Experimental configuration for screening experiments was similar but without gas-phase sampling and with different flows and sample sizes (see text). 


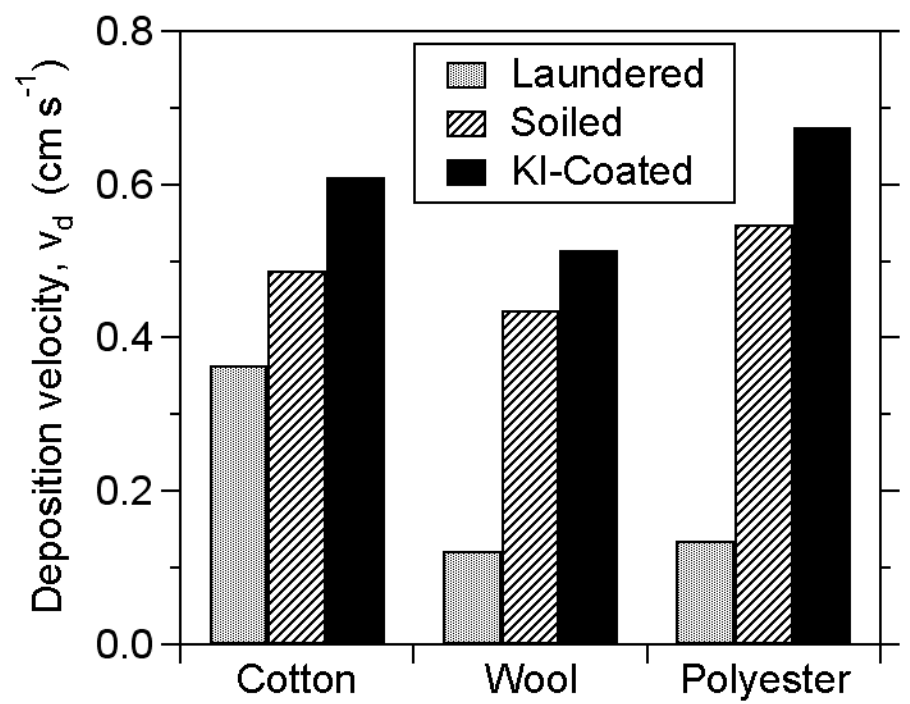

Figure 3-3: Ozone deposition velocity (3-h average) to three common fabrics: cotton, wool, and polyester. The "laundered" sample was washed a few days prior to being exposed to ozone, the "soiled" sample was worn near the skin for $8 \mathrm{~h}$ prior to exposing it to ozone, and the "KI-coated" sample represents the maximum reactivity of a material for the given experimental conditions. 


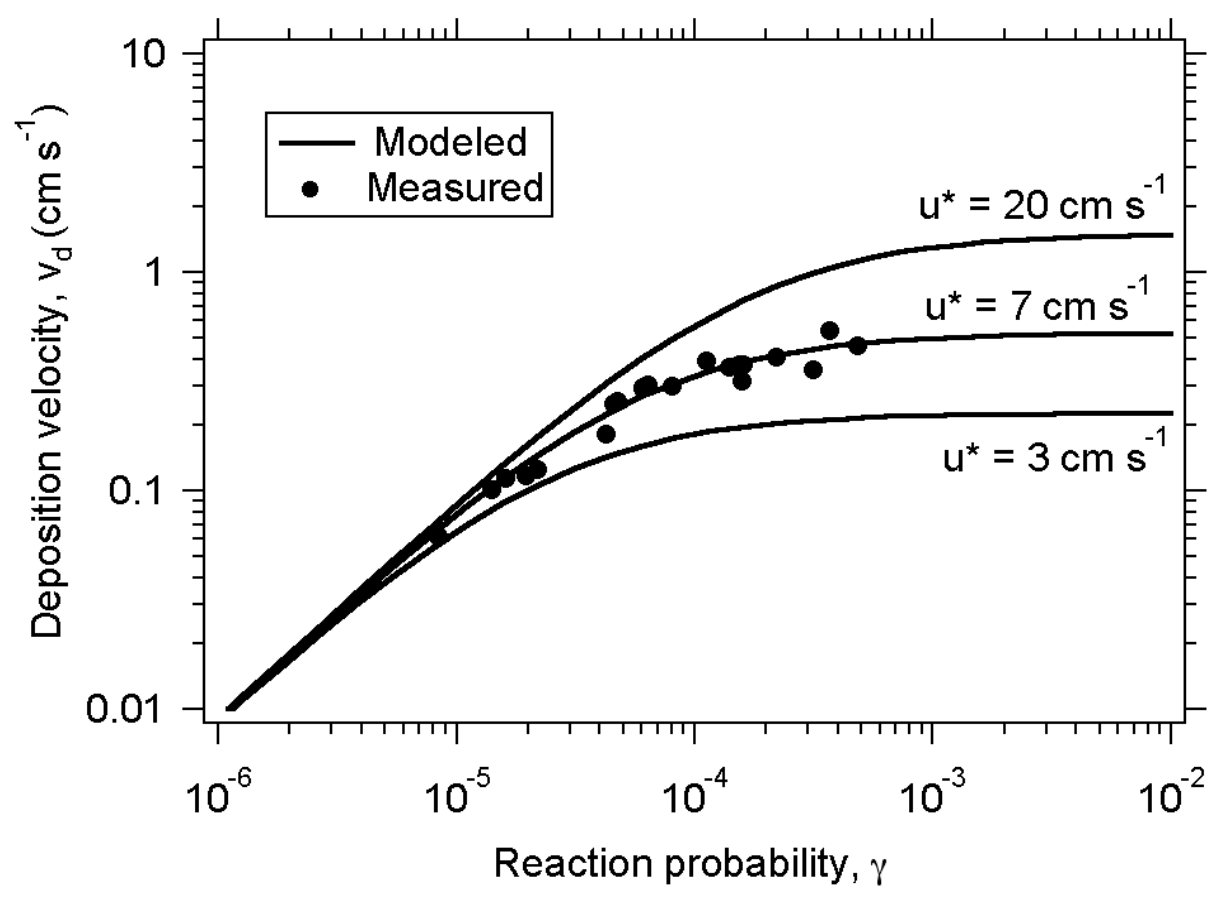

Figure 3-4: Screening experiment data mapped onto deposition velocity model developed by Morrison and Nazaroff (2002b). 


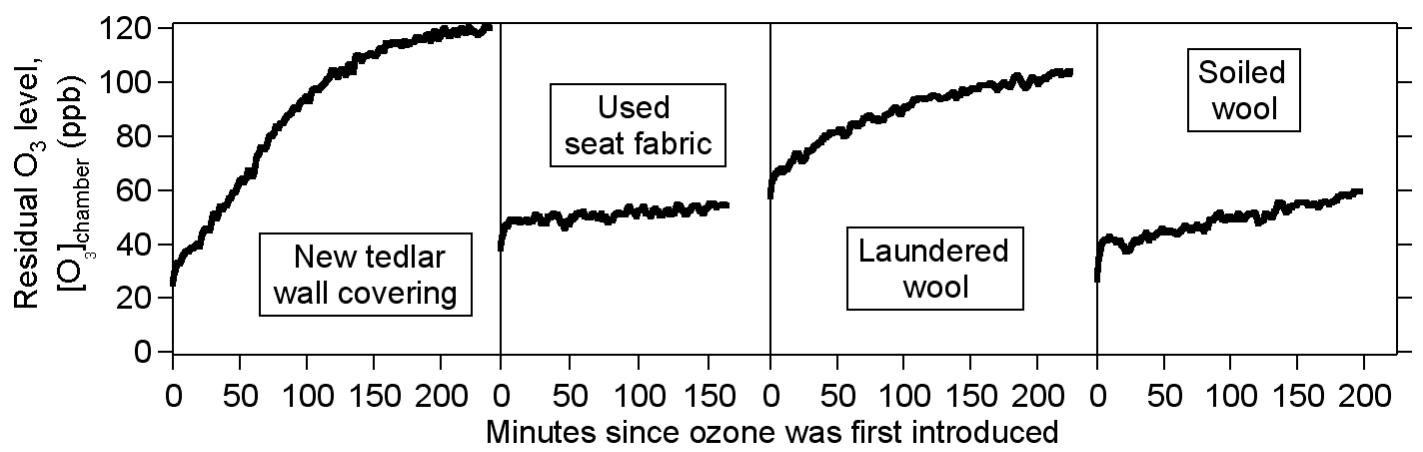

Figure 3-5: Ozone reactivity profile of four materials exposed to an identical set of conditions. Residual ozone level is the ozone level measured at the exhaust of the chamber; the supply level was $120 \mathrm{ppb}$. 

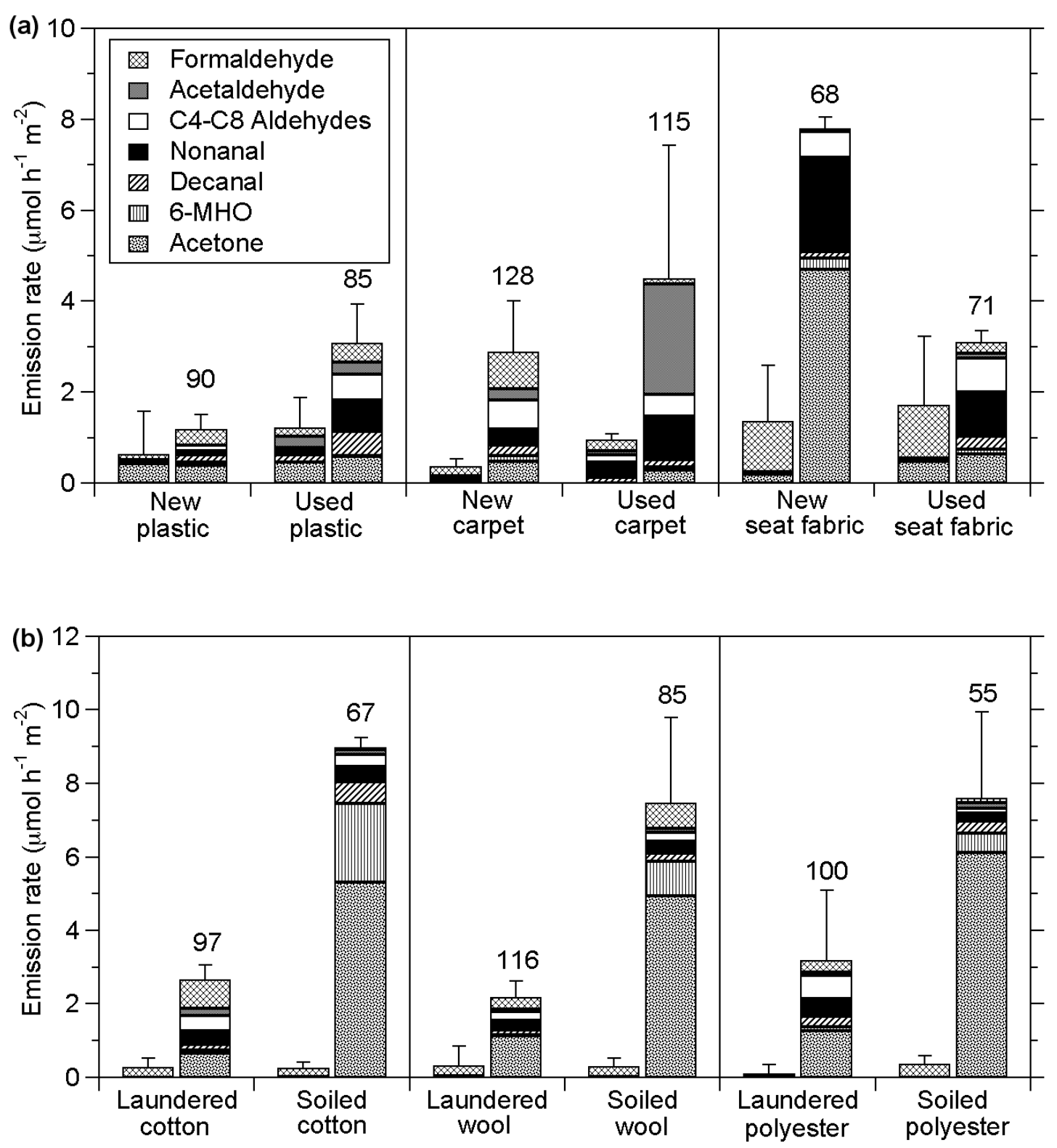

Figure 3-6: Emission rates of selected volatile organic compounds from (a) new and used common cabin materials and (b) laundered and soiled clothing fabrics. For each material presented, the left bar represents the average emissions without ozone during a 180minute conditioning period (no ozone), and the right bar represents the average emissions during the initial 90-minute ozone exposure period. The number above the right bar is the 90-minute average residual ozone concentration in ppb; the supply air concentration was always $160 \mathrm{ppb}$. Error bar indicates plus one standard deviation from analysis of replicate integrated samples. 


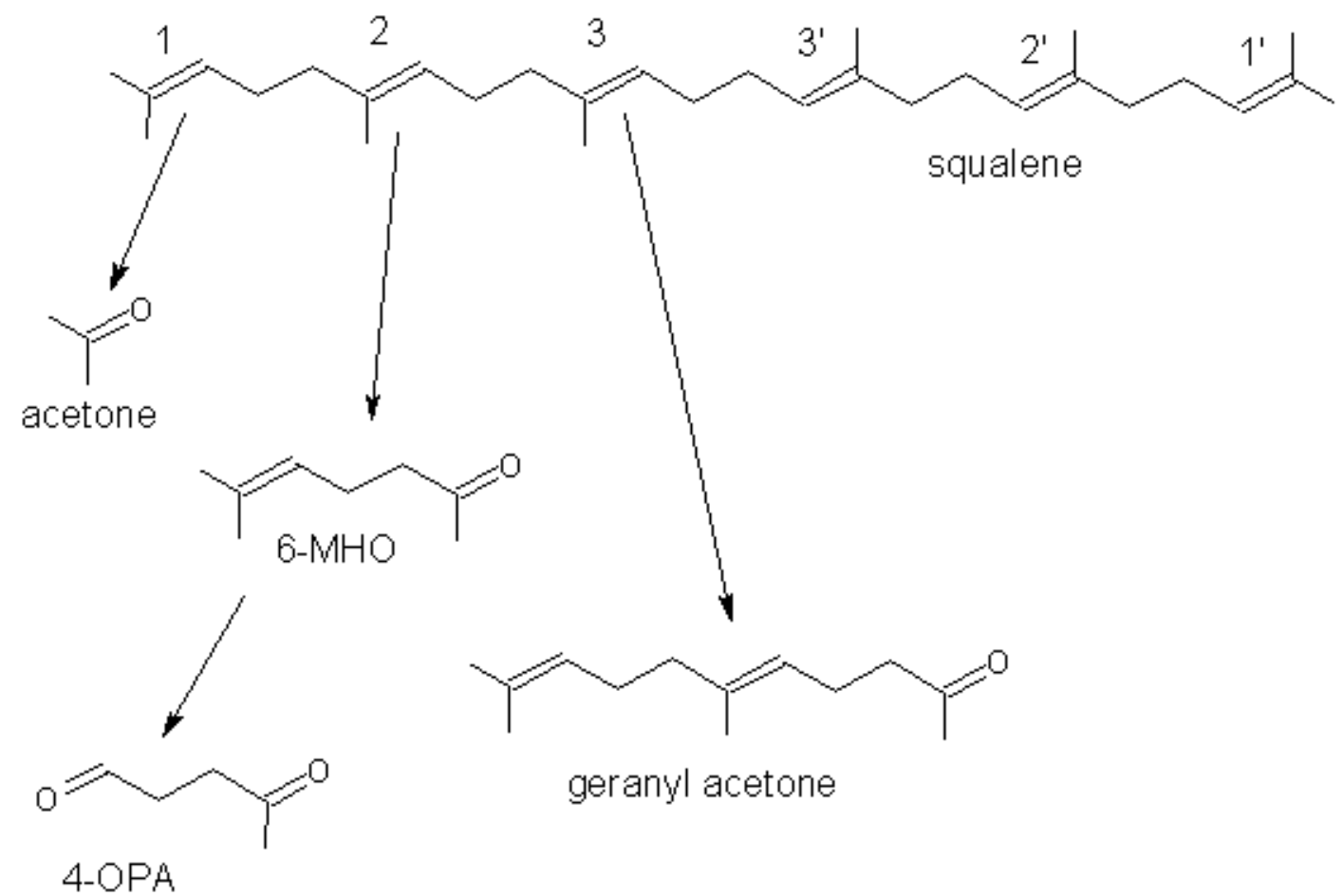

Figure 3-7: Squalene and its detected, volatile oxidation products, adapted from Fruekilde et al. (1998) and Wells et al. (2008). Squalene has six symmetric double bonds, which are numbered in the figure. If ozone reacts with the first double bond acetone is formed, with the second 6-MHO is formed, and with the third geranyl acetone is formed.

Complementary semivolatile compounds are formed from each of these reactions. Any byproduct that contains a double bond can be further oxidized to form more oxygenated byproducts. For example, 6-MHO, geranyl acetone, and potentially other semivolatile squalene products can be further oxidized to form 4-OPA (only 6-MHO oxidation is illustrated here). The hydroxyl radical $(\mathrm{OH})$ may be formed from ozone reactions, and $\mathrm{OH}$ may also react with squalene and its products. 


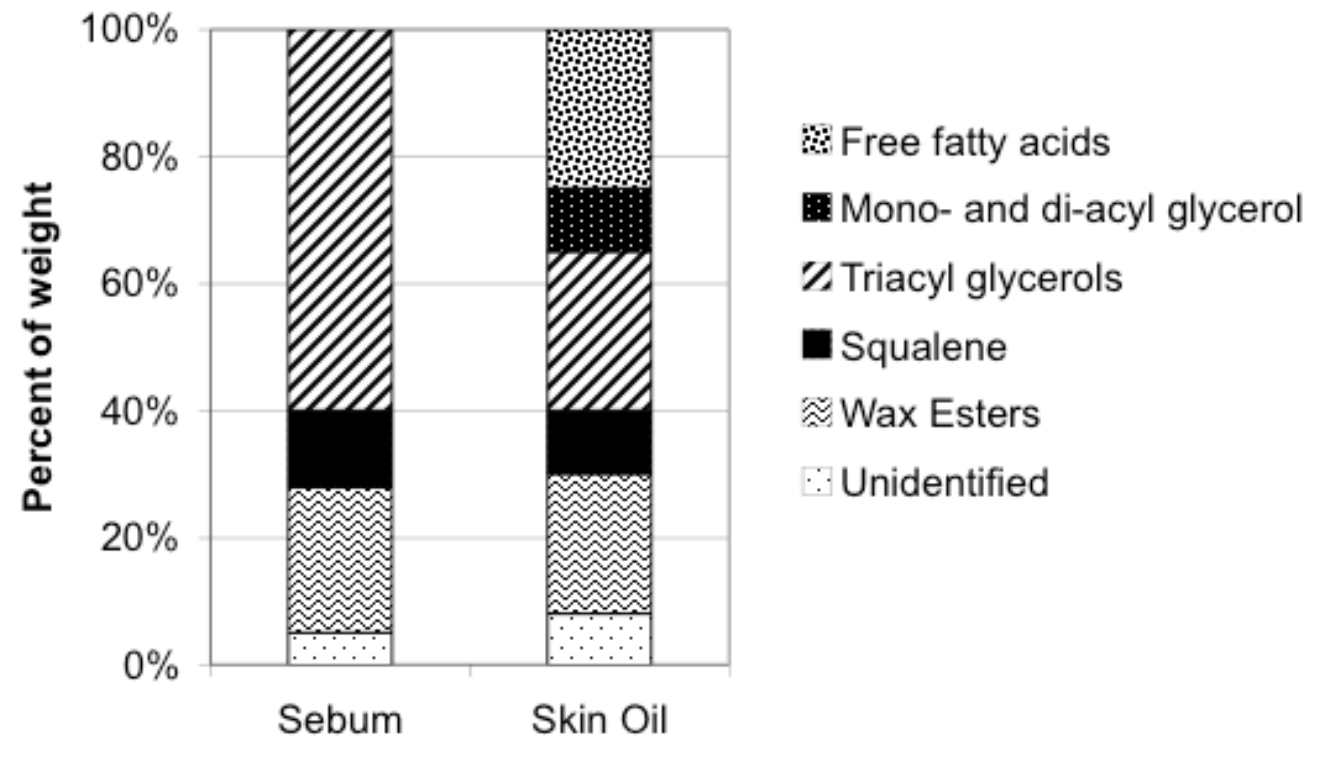

Figure 3-8: Composition of sebum and skin oil (Nicolaides, 1974). Sebum is the material produced by the sebaceous gland. Hydrolysis of the triacyl glycerols occurs in gland ducts and on the skin, resulting in a different composition of skin oil. 


\begin{tabular}{|c|c|c|c|}
\hline Constituent & $\%$ Weight & Name & $\begin{array}{c}\text { hexadecanoic acid; } \\
\text { palmitic acid }\end{array}$ \\
\hline $\begin{array}{c}\mathrm{C}_{16} \text { fatty } \\
\text { acid }\end{array}$ & $20.8 \%$ & $\begin{array}{c}\text { cis-hexadec-6- } \\
\text { enoic acid }\end{array}$ \\
\hline $\begin{array}{c}\mathrm{C}_{16: 1 \Delta 6} \text { fatty } \\
\text { acid }\end{array}$ & $17.8 \%$ & $\begin{array}{c}\text { cis-octadec-8-enoic } \\
\text { acid }\end{array}$ \\
\hline $\begin{array}{c}\mathrm{C}_{18: 1 \Delta 8} \text { fatty } \\
\text { acid }\end{array}$ & $7.2 \%$ & $\begin{array}{c}\text { tetradecanoic acid; } \\
\text { myristic acid }\end{array}$ \\
\hline $\begin{array}{c}\mathrm{C}_{14} \text { fatty } \\
\text { acid }\end{array}$ & $5.6 \%$ \\
\hline $\begin{array}{c}\text { Iso-15- } \\
\text { fatty acid }\end{array}$ & $3.3 \%$ & $\begin{array}{c}\text { methylpentadec-6- } \\
\text { enoic acid }\end{array}$ & \\
\hline
\end{tabular}

Figure 3-9: Most prevalent fatty acids in human skin oil, summarized from Nicolaides (1974) and Pandrangi and Morrison (2008). The column "\% weight" indicates the contribution of the indicated fatty acid to total weight of fatty acids in skin oil. Fatty acids constitute $\sim 25 \%$ of skin oil. 


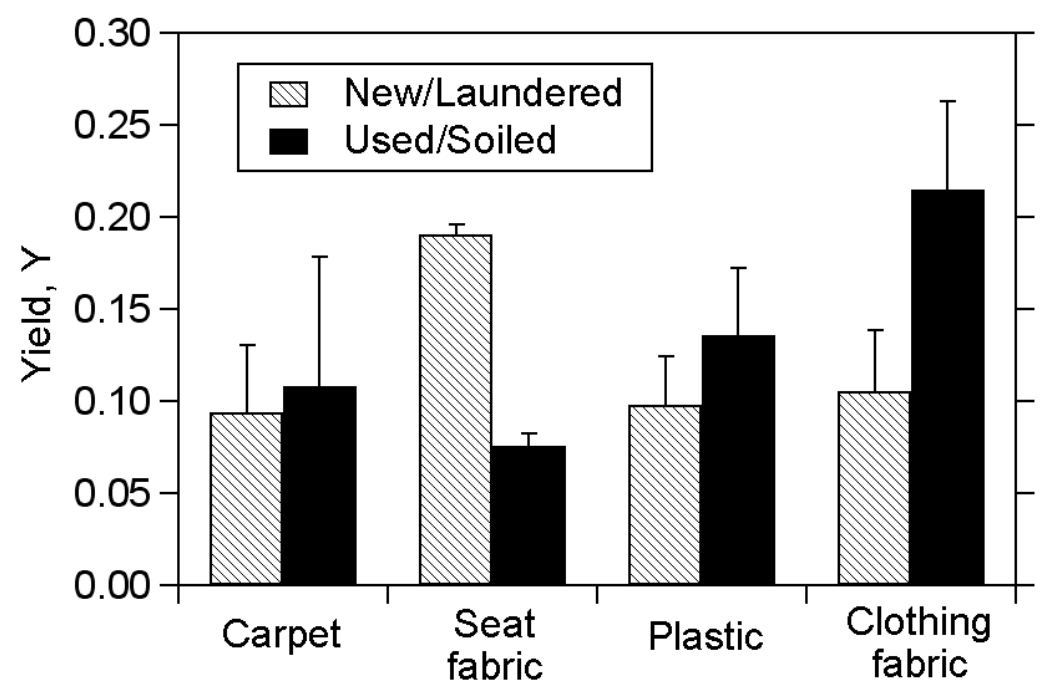

Figure 3-10: Molar yields of the sum of $\mathrm{C}_{1}$ through $\mathrm{C}_{10}$ saturated aldehydes, acetone, and 6-MHO from new and used cabin materials and laundered and soiled clothing fabrics during first 90 minutes of exposure to $160 \mathrm{ppb}$ ozone (supply level) at 10\% RH. 


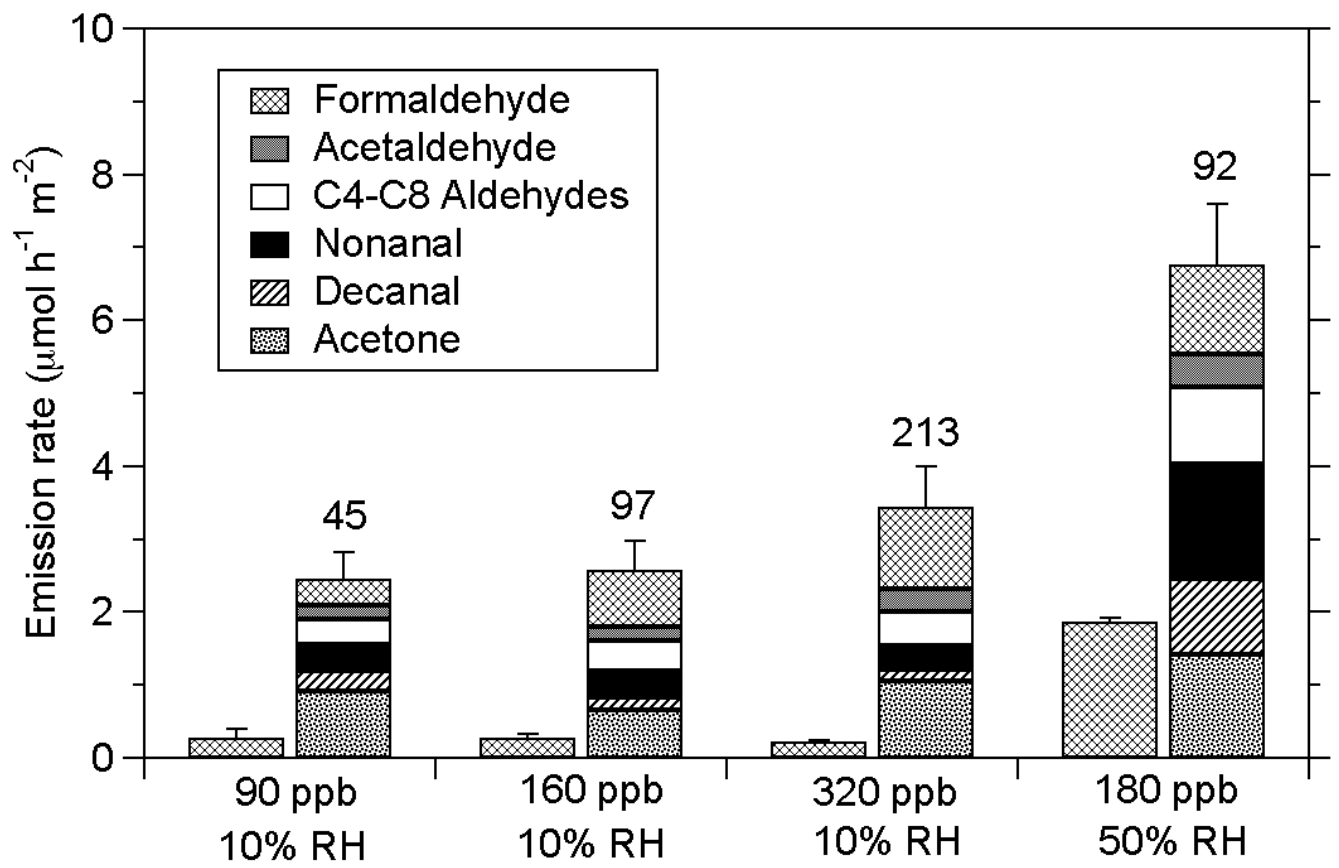

Figure 3-11: Emission rates from cotton exposed to varying levels of ozone under different relative humidity conditions. For each material, the left bar represents the average emission rates without ozone during a 180 -minute conditioning period (no ozone), and the right bar represents the average emissions during the initial 90-minute ozone exposure period. The number above the right bar is the 90-minute average residual ozone concentration in ppb; the supply air concentration is part of the $\mathrm{x}$-axis label. Error bar indicates plus one standard deviation from analysis of replicate integrated samples. 


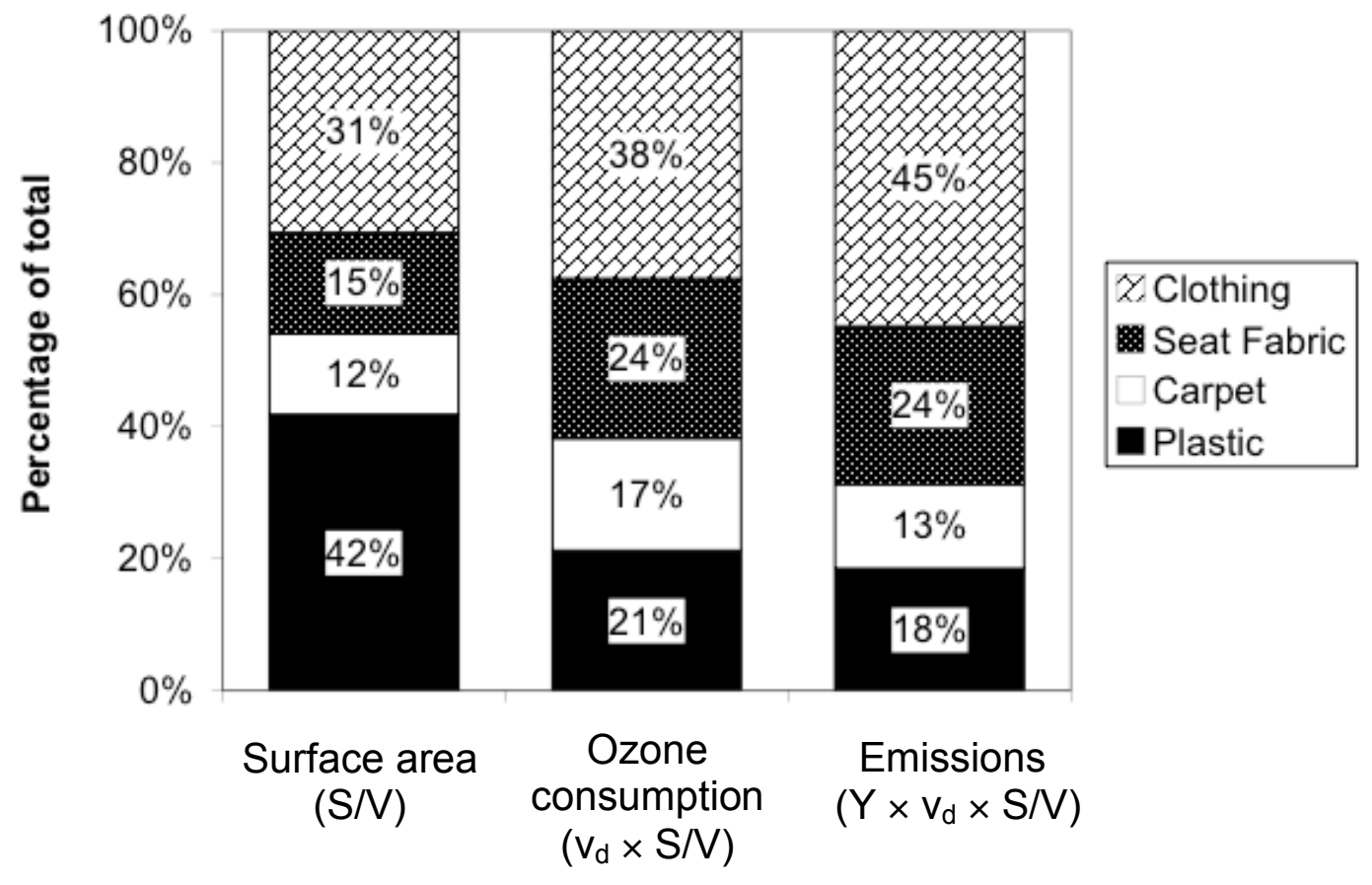

Figure 3-12: Contribution of major cabin surfaces to (a) total cabin surface area, (b) ozone consumption, and (c) byproduct emissions. Surface areas are estimated for a fully occupied Boeing 737 plane; see Section 3.B. for cabin S/V ratios. Data used to make this plot are presented in Table $3 \mathrm{C}-3$. 


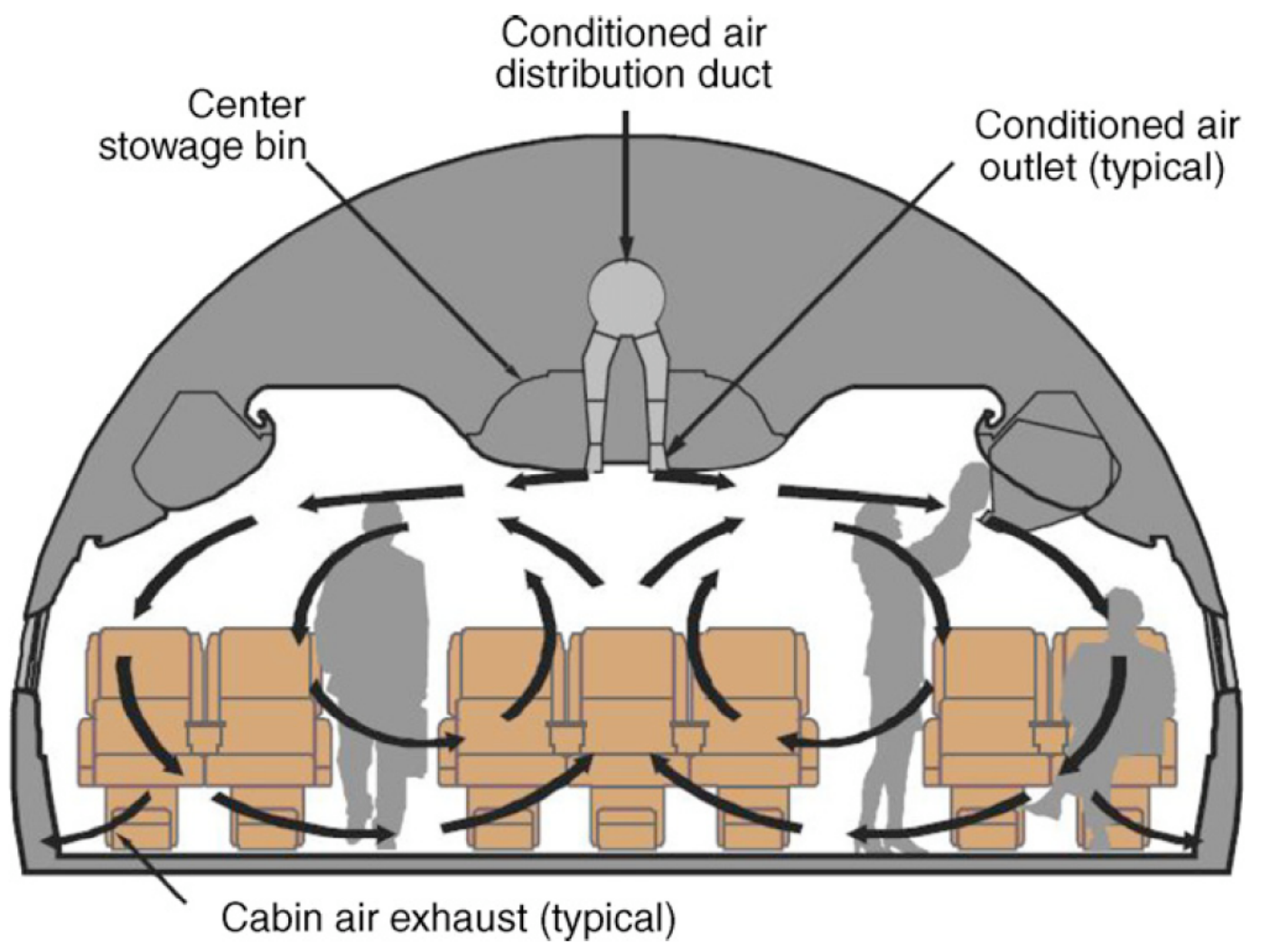

Figure 3-13: Typical airflow patterns in a wide-body plane (www.boeing.com). 

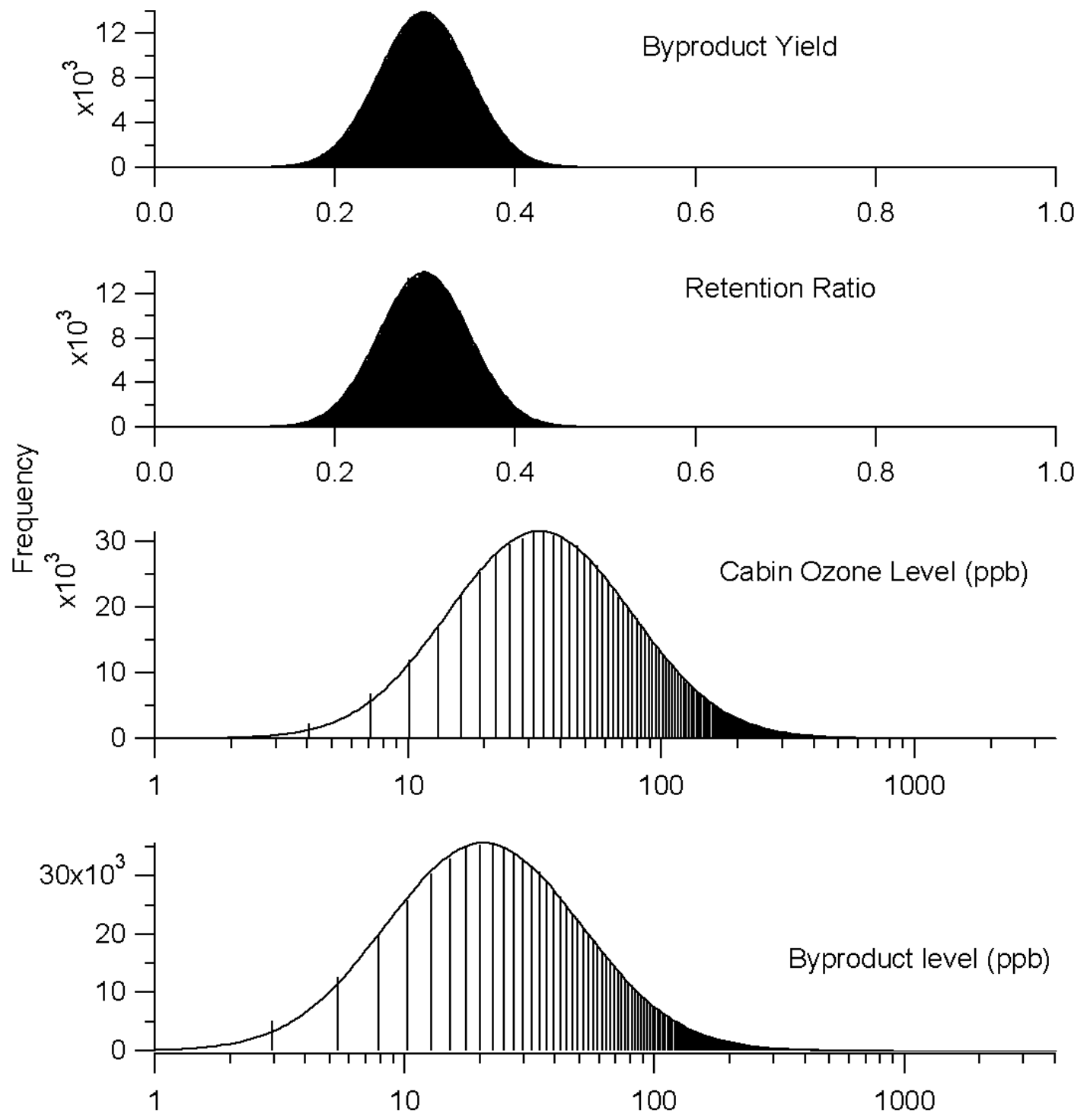

Figure 3-14: Input (yield, retention ratio, and cabin ozone level) and output distributions for Monte Carlo simulation of peak-hour byproduct levels. 


\section{A. Appendix: Extrapolating from chamber data to real environments}

In this appendix, the reader is guided through the process of taking ozone deposition and byproduct yield data gathered in chamber experiments and extrapolating to a real environment, in this case, the aircraft cabin environment. The process employs the model developed by Morrison and Nazaroff (2002b), which combines the mathematical model developed by Cano-Ruiz et al. (1993) and the expression developed by Lai and Nazaroff (2000) for turbulent mass transport of particles near surfaces to form an expression for reactive gas uptake on surfaces (equation 3-7). Figure 3A-1 shows the steps to determine the deposition velocity for an individual material in a real

environment, and Figure 3A-2 shows the steps to determine the individual and overall byproduct concentrations in the real environment owing to ozone reactions with all surfaces. 


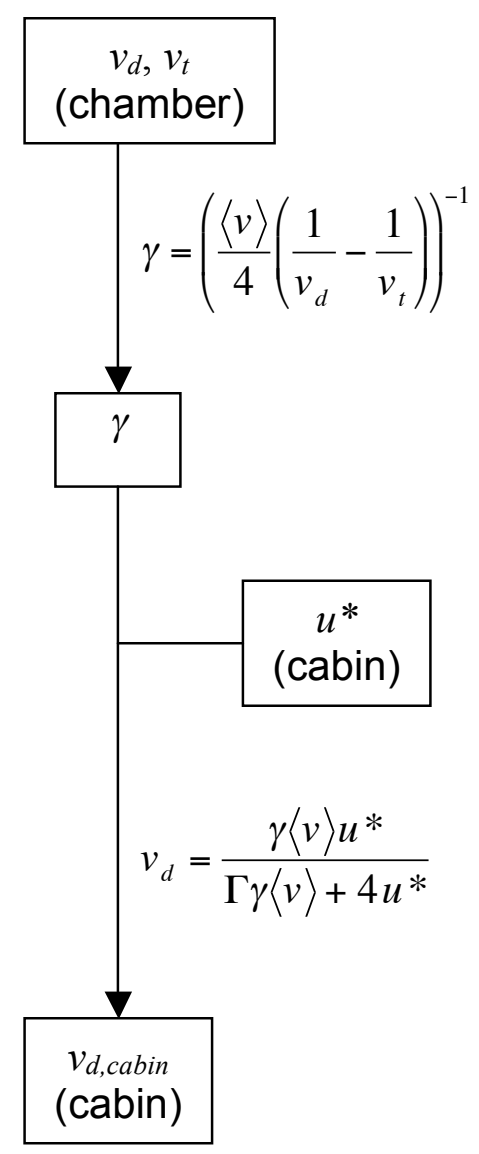

STEP 1: Measure deposition velocity and transport-limited deposition velocity, $v_{d}$ and $v_{t}$, for a material in the chamber (see Section 3.2.1).

STEP 2: Calculate reaction probability, $\gamma$, for that material using equation 3-4.

STEP 3: Determine friction velocity, $u^{*}$, in the real environment of concern from the literature or measurements.

STEP 4: Calculate deposition velocity to a surface in the real environment using equation 3-7. Boltzmann velocity, $\langle v\rangle$, is equal to $3.6 \times 10^{4} \mathrm{~cm} \mathrm{~s}^{-1}$ for ozone in typical indoor conditions, and $\Gamma$ can be determined from a numerical integration but is equal to 13.3 for indoor conditions (equation 11, Morrison and Nazaroff, 2002b).

Figure 3A-1: Steps to calculate the ozone deposition velocity in a real environment given chamber data and information about flow conditions in the real environment. 


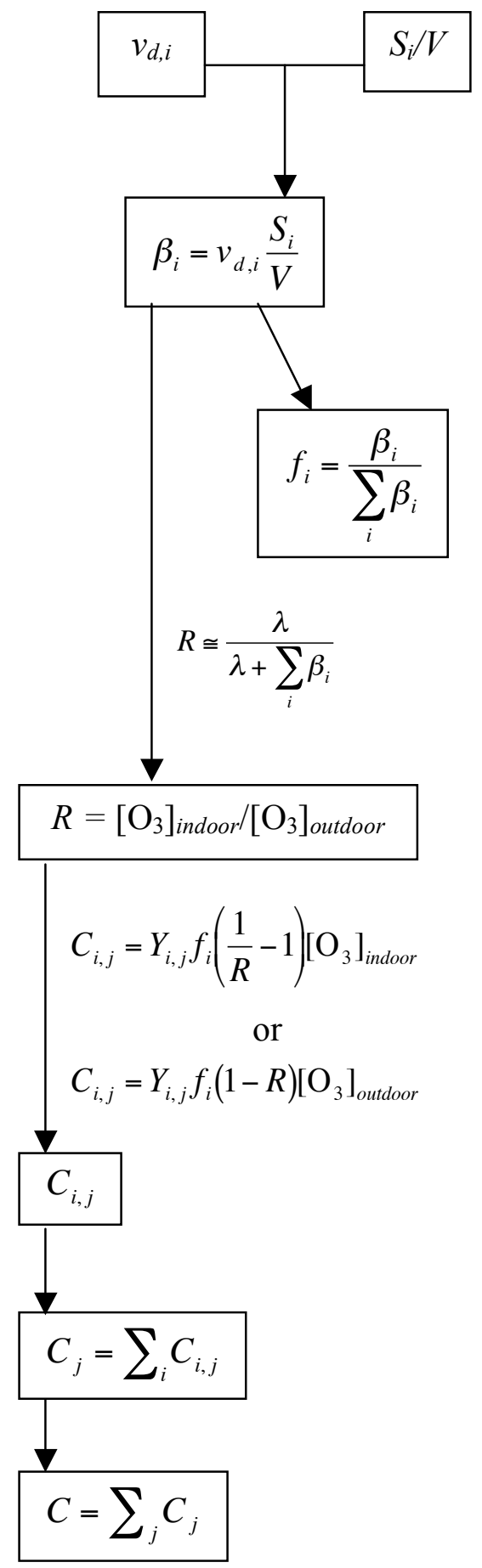

STEP 1: Determine deposition velocities for all of the materials in the real environments using the process shown in Figure 3A-1. Here $i$ is the surface index.

STEP 2: Determine the surface-to-volume ratio, $S_{i} / V$, for each surface using measurements or values reported in the literature.

STEP 3: The pseudo first-order lost rate coefficient for each material, $\beta_{i}$, is calculated as the product of deposition velocity and surface-to-volume ratio.

STEP 4: The fraction of ozone that is consumed by reaction with each surface is the ratio of $\beta_{i}$ to the sum of the first-order loss rates to all surfaces.

STEP 5: If the real environment is assumed to be well-mixed and at steady-state, reasonable assumptions for estimation purposes, then the overall indoor-to-outdoor ratio of ozone (called the retention ratio, $R$, for the cabin) can be calculated using equation 3-1.

STEP 6: The level of byproduct $j$ owing to ozone reaction with surface $i, C_{i, j}$, is calculated by multiplying the yield for that byproduct and surface, $Y_{i, j}$, with the amount of ozone consumed by a surface, which is equal to $f_{i}(1 / R-1)\left[\mathrm{O}_{3}\right]_{\text {indoor }}$ when the indoor ozone level is known, and $f_{i}(1-R)\left[\mathrm{O}_{3}\right]_{\text {outdoor }}$ when only the outdoor ozone level is known (assuming no ozone converter in the airplane's ventilation system).

STEP 7: Summing over all surfaces will give the total level of byproduct $j, C_{j}$, and then summing over all compounds will give the total byproduct level owing to ozone reactions with surfaces, $C$.

Figure 3A-2: Steps to calculate the ozone byproduct concentration using deposition velocities and surface-to-volume ratios of individual materials. 


\section{B. Appendix: Surface-to-volume ratios for various aircraft cabins}

Surface areas and surface-to-volume ratios $(\mathrm{S} / \mathrm{V})$ are important parameters for estimating ozone uptake and byproduct formation. An analysis of different plane types was performed to determine the range of surface-to-volume ratios for passenger aircraft cabins and the results are presented in this appendix.

\section{B.1. Aircraft types}

There are two main types of planes that service moderate to long routes for commercial carriers. Wide-body planes have two aisles and are typically used for longdistance travel. Narrow body planes have one aisle and are typically used for shorterdistance travel. The following three cabin types were considered. Their dimensions are summarized in Table 3B-1, and the method of analysis is described in the following paragraphs.

- Boeing 777 (wide-body)

- MD-80 (narrow-body)

- Boeing 737 (narrow-body)

\section{B.2. Surface categories}

Five main surfaces categories were chosen: carpet, plastic, seat fabric, surfaces associated with passengers (skin and clothing), and "other". Plastic includes the wall coverings, overhead storage bins, windows, tray tables, and overhead controls. The "other" category includes bottoms of seats, hand rests, and structural supports for the seats. Only surfaces for which deposition and emissions experiments were conducted 
(carpet, plastic, seat fabric, and clothing) were included in Figure 3-12. The category "other" was added here to include elements that were not tested in chamber experiments.

\section{B.3. Dimensions and parameters}

The inventory of the surfaces of a B737-800 was completed in 2006 using information from the Boeing website and from a Boeing employee. The three-digit number after the plane model is a code for the length of the plane; surface-to-volume ratios are essentially the same regardless of the length of the plane, so long as the body type is the same and the seating arrangement is similar. The curvilinear cross-sectional length of the plastic (including walls, ceilings, and bins), which is referred to as "plastic perimeter" in Table 3B-1, was determined to be $9.1 \mathrm{~m}$ by communication with a Boeing employee, and confirmed using scale drawings of the plane cross-section from www.boeing.com. The interior cross-sectional area of the plane was determined from of

scale-drawings to be $4.7 \mathrm{~m}^{2}$. The cross-sectional width of carpet was determined to be 3.2 $\mathrm{m}$. In a recent analysis in 2008, the dimensions of a B777 and MD-80 were determined entirely from scale drawings from www.boeing.com, and parameter values are given in Table 3B-1.

The plastic features of the plane are three-dimensional, and "plastic perimeter" only gives a two-dimensional measure. For all three plane types the value for plastic perimeter was multiplied by a factor of 1.2 to estimate the three-dimensional surface area.

Note that dividing "plastic perimeter" or "carpet width" by the cross-sectional area of the plane yields the correct units for surface-to-volume ratio, but it underestimates $\mathrm{S} / \mathrm{V}$ because the volume of the seats and passengers is not accounted for when using the 
cross-sectional values. "Plastic perimeter" and "carpet width" were multiplied by the length of the passenger area and then divided by the appropriate volume. When considering an unoccupied plane, the interior volume of the fuselage minus the volume of the seats (referred to as "volume with seats" in Table 3B-1) was used to determine surface-to-volume ratio. In the case of a fully occupied cabin, the volume used for $\mathrm{S} / \mathrm{V}$ calculations was the "volume with passengers and seats", which is the interior volume of the fuselage minus the volume of the seats and the passengers. The volume of the seats is equal to the volume of one seat, $0.08 \mathrm{~m}^{3}$, multiplied by the number of seats. The average volume of a passenger was assumed to be $0.08 \mathrm{~m}^{3}$, and the volume of passengers was determined by multiplying the average volume a passenger by the number of passengers, which is equal to the number of seats for a fully occupied plane.

The surface area of single seat was determined by measuring the amount of fabric in a seat cover obtained from a major airline; the total area was $1.2 \mathrm{~m}^{2}$, which includes the sides of the chair (which may not be exposed) and excludes the bottom of the seat because it is not covered in seat fabric. Thus, when the plane is unoccupied, the total amount of seat fabric is equal to the number of seats multiplied by $1.2 \mathrm{~m}^{2}$. When occupied, the half of the seat fabric was assumed to be covered by the passenger, and so the total area of seat fabric is equal to $0.6 \mathrm{~m}^{2}$ times the number of seats for a fully occupied plane.

Each person was assumed to have $1.8 \mathrm{~m}^{2}$ of surface area, contributing $1.3 \mathrm{~m}^{2}$ of exposed surface when seated. Thus the total passenger surface area is equal to $1.3 \mathrm{~m}^{2}$ times the number of seats when the plane is fully occupied. 


\section{B.4. Conclusion}

The new analysis of a narrow-body plane (MD-80) using scale diagrams alone was similar to the previous analysis of different narrow-body plane (B737). As expected, the wide-body planes had lower total $\mathrm{S} / \mathrm{V}$ ratios. This is predominantly because there is more plastic surface per volume in a smaller plane. The S/V ratios for seat fabric and passengers were also slightly lower for the wide-body plane. The $\mathrm{S} / \mathrm{V}$ ratios for carpet were similar for all planes, $\sim 0.6 \mathrm{~m}^{2} \mathrm{~m}^{-3}$. The calculation of seat fabric and passenger area is sensitive to the seating arrangement and the type of seats used; higher density (and thus more economical) seating arrangements involve smaller but more closely spaced seats, which increase the overall $\mathrm{S} / \mathrm{V}$ ratio. Based on the trends shown here, the retention ratio (equation 3-1) is expected to be higher (i.e. ozone consumption would be lower), and therefore byproduct formation would be lower in larger planes, all else being equal.

The surface-to-volume ratios for carpet, plastic, and "other" increased slightly from the unoccupied to the occupied condition because the open volume of the plane decreases slightly when the plane is occupied.

Limited evidence has been published characterizing surface-to-volume ratios in buildings. One study of sorption dynamics in a few residences indicates that $\mathrm{S} / \mathrm{V}$ ratios

are in the range of $3-4.5 \mathrm{~m}^{-1}$ (Singer et al., 2007). This is similar to the value calculated for the larger plane. 
Table 3B-1: Cabin dimensions and surface-to-volume calculations for various aircraft. (See footnotes on next page.)

\begin{tabular}{cccc}
\hline Dimension & $\begin{array}{c}\text { Wide-body } \\
\text { B777 }\end{array}$ & $\begin{array}{c}\text { Narrow- } \\
\text { body } \\
\text { MD-80 }\end{array}$ & $\begin{array}{c}\text { Narrow- } \\
\text { body } \\
\text { B737 }\end{array}$ \\
\hline \hline & & \\
Internal fuselage dimensions & \\
\hline cross-sectional area $\left(\mathrm{m}^{2}\right)$ & 11.6 & 5.4 & 4.7 \\
volume $\left(\mathrm{m}^{3}\right)$ & 618 & 126 & 116 \\
volume with seats $\left(\mathrm{m}^{3}\right)$ & 578 & 114 & 103 \\
volume with passengers and & 534 & 102 & 92 \\
seats $\left(\mathrm{m}^{3}\right)$ & 53 & 23 & 21 \\
length of passenger area $(\mathrm{m})$ & & & \\
Passenger & and seat dimensions & 140 \\
seats and passengers & 500 & 150 & 1.2 \\
fabric per seat $\left(\mathrm{m}^{2}\right)$ & 1.2 & 1.2 & 0.08 \\
volume per seat $\left(\mathrm{m}^{3}\right)$ & 0.08 & 0.08 & 0.5 \\
"other" surface per seat $\left(\mathrm{m}^{2}\right)^{\mathrm{a}}$ & 0.5 & 0.5 & 1.8 \\
area of person $\left(\mathrm{m}^{2}\right)$ & 1.8 & 1.8 & 1.3 \\
area of person exposed $\left(\mathrm{m}^{2}\right)$ & 1.3 & 1.3 & 0.08 \\
volume of person $\left(\mathrm{m}^{3}\right)$ & 0.08 & 0.08 &
\end{tabular}

Carpet and plastic dimensions

\begin{tabular}{cccc}
\hline carpet width $(\mathrm{m})$ & 5.8 & 2.9 & 3.2 \\
plastic perimeter $(\mathrm{m})$ & 10.4 & 8.5 & 9.1
\end{tabular}

\begin{tabular}{cccc}
\multicolumn{4}{c}{ Surface-to-volume ratios, without passengers $\left(\mathrm{m}^{-1}\right)^{b}$} \\
\hline plastic $^{\mathrm{c}}$ & 1.1 & 2.1 & 2.2 \\
carpet $^{\mathrm{c}}$ & 0.5 & 0.6 & 0.6 \\
seat fabric $^{\mathrm{d}}$ & 1.0 & 1.6 & 1.6 \\
other $^{\mathrm{d}}$ & 0.4 & 0.7 & 0.7 \\
\hline total & 3.1 & 4.9 & 5.1
\end{tabular}

Surface-to-volume ratios, without passengers $\left(\mathrm{m}^{-1}\right)^{e}$

\begin{tabular}{cccc}
\hline plastic $^{\mathrm{c}}$ & 1.2 & 2.3 & 2.5 \\
carpet $^{\mathrm{c}}$ & 0.6 & 0.7 & 0.7 \\
seat fabric $^{\mathrm{f}}$ & 0.6 & 0.9 & 0.9 \\
passengers $^{\mathrm{g}}$ & 1.2 & 1.9 & 1.9 \\
other $^{\mathrm{d}}$ & 0.5 & 0.7 & 0.7 \\
\hline total & 4.1 & 6.5 & 6.8
\end{tabular}


Table 3B-1 (cont.): Cabin dimensions and surface-to-volume calculations for various aircraft.

a "Other surfaces per seat" include the hand rests, the seat bottom, and the structural supports for the seat.

${ }^{\mathrm{b}}$ Surface-to-volume ratio calculated using "volume with seats".

"Multiply "carpet width" or "plastic perimeter" by length of passenger area and divide by appropriate volume. The "plastic perimeter" is two-dimensional estimate of nominal plastic surface area. Three-dimensional area was estimated as $20 \%$ higher (i.e. "plastic perimeter" was multiplied by 1.2 for these calculations.)

d Multiply "fabric per seat" or "other surface per seat" by number of seats and divide by appropriate volume.

e Surface-to-volume ratio calculated using "volume with passengers and seats".

f "Fabric per seat" was multiplied by 0.5 for the B777, B737, and MD-80.

g Multiply "area of person exposed" by the number of passengers and divide by the "volume with passengers and seats". 


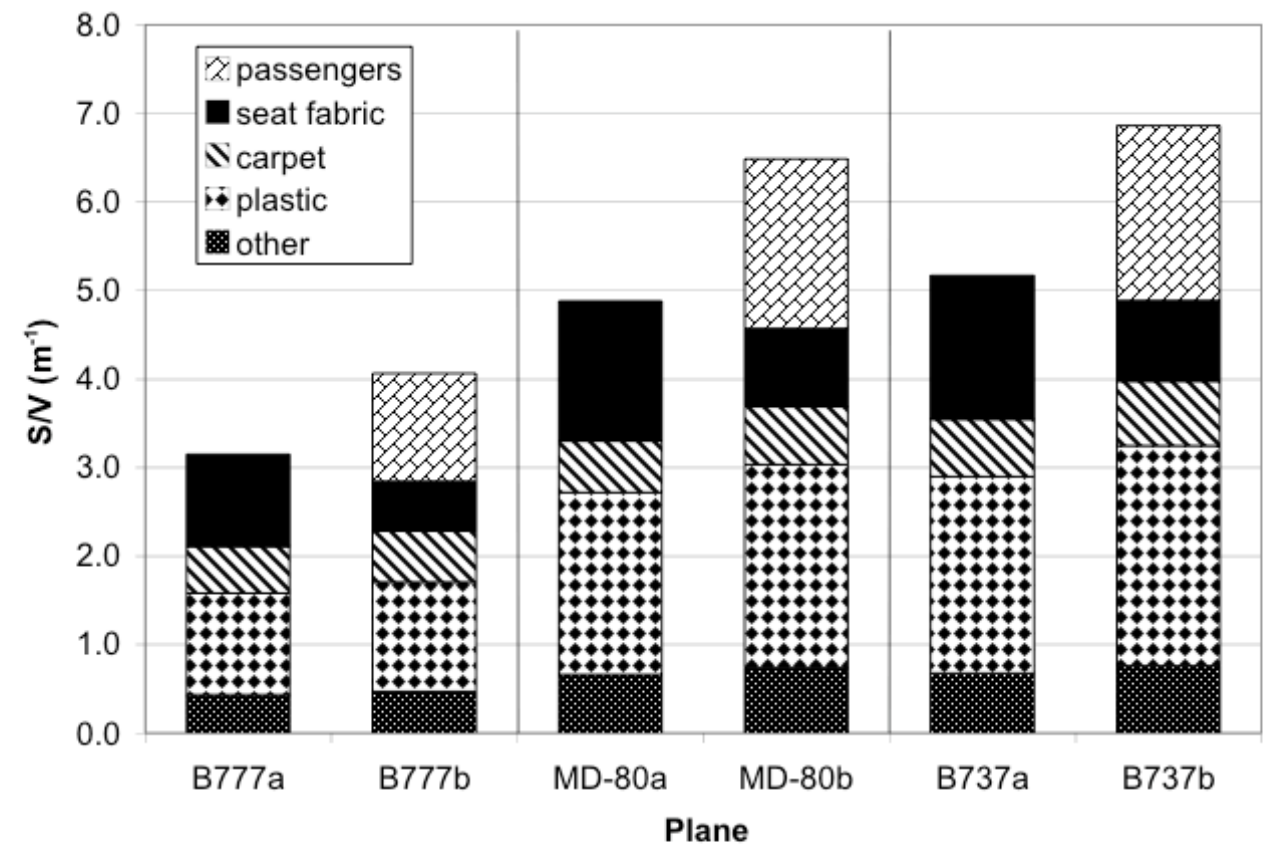

Figure 3B-1: Surface-to-volume ratios for a Boeing 777 (wide-body plane), an MD-80 (narrow-body plane), and a Boeing 737 (narrow-body plane). For all of the planes two bars are shown: (a) indicates an unoccupied plane, and (b) indicates a fully occupied plane. 


\section{C. Appendix: Additional experimental data}

Table 3C-1: Yields ${ }^{\mathrm{a}}$ of oxidation byproducts from laundered cotton in various conditions during 90-minute ozone exposure. ${ }^{\mathrm{b}}$

\begin{tabular}{|c|c|c|c|c|c|c|}
\hline Compound & $\begin{array}{c}\text { Cotton } \\
10 \% \mathrm{RH} \\
90 \mathrm{ppb}\end{array}$ & $\begin{array}{l}\text { Cotton } \\
10 \% \mathrm{RH} \\
160 \mathrm{ppb} \\
\text { (Base case) }\end{array}$ & $\begin{array}{l}\text { Cotton } \\
10 \% \mathrm{RH} \\
320 \mathrm{ppb}\end{array}$ & $\begin{array}{l}\text { Cotton } \\
50 \% \mathrm{RH} \\
180 \mathrm{ppb}\end{array}$ & $\begin{array}{l}\text { Cotton } \\
10 \% \mathrm{RH} \\
160 \mathrm{ppb} \\
\text { (Triplicate) }\end{array}$ & $\begin{array}{c}\text { Cotton } \\
10 \% \mathrm{RH} \\
160 \mathrm{ppb} \\
\text { (Duplicate) }\end{array}$ \\
\hline Formaldehyde & 0.005 & 0.023 & 0.020 & 0.000 & 0.017 & 0.018 \\
\hline Acetaldehyde & 0.010 & 0.010 & 0.007 & 0.011 & 0.014 & 0.006 \\
\hline Acetone & 0.045 & 0.033 & 0.024 & 0.039 & 0.057 & 0.018 \\
\hline Pentanal & 0.002 & 0.003 & 0.001 & 0.003 & 0.001 & 0.001 \\
\hline Hexanal & 0.006 & 0.009 & 0.004 & 0.011 & 0.004 & 0.004 \\
\hline Heptanal & 0.004 & 0.005 & 0.002 & 0.007 & 0.002 & 0.003 \\
\hline Octanal & 0.004 & 0.004 & 0.002 & 0.006 & 0.003 & 0.003 \\
\hline Nonanal & 0.019 & 0.018 & 0.007 & 0.040 & 0.013 & 0.015 \\
\hline Decanal & 0.011 & 0.008 & 0.003 & 0.025 & 0.007 & 0.010 \\
\hline Total & 0.106 & 0.113 & 0.072 & 0.142 & 0.117 & 0.078 \\
\hline
\end{tabular}


Table 3C-2: Yields ${ }^{\mathrm{a}}$ of oxidation byproducts from new and used cabin materials (upper table) and laundered and soiled clothing fabrics (lower table). ${ }^{\mathrm{b}}$

\begin{tabular}{|c|c|c|c|c|c|c|}
\hline Compound & $\begin{array}{c}\text { New } \\
\text { Plastic }\end{array}$ & $\begin{array}{c}\text { Used } \\
\text { Plastic }\end{array}$ & $\begin{array}{c}\text { Used } \\
\text { Carpet }\end{array}$ & $\begin{array}{c}\text { New } \\
\text { Carpet }\end{array}$ & $\begin{array}{c}\text { New } \\
\text { Seat } \\
\text { Fabric } \\
\end{array}$ & $\begin{array}{c}\text { Used } \\
\text { Seat } \\
\text { Fabric } \\
\end{array}$ \\
\hline Formaldehyde & 0.019 & 0.011 & 0.000 & 0.020 & 0.000 & 0.000 \\
\hline Acetaldehyde & 0.000 & 0.000 & 0.058 & 0.008 & 0.000 & 0.002 \\
\hline Acetone & 0.000 & 0.005 & 0.010 & 0.019 & 0.113 & 0.004 \\
\hline Pentanal & 0.000 & 0.002 & 0.000 & 0.002 & 0.000 & 0.001 \\
\hline Hexanal & 0.003 & 0.005 & 0.003 & 0.004 & 0.005 & 0.006 \\
\hline Heptanal & 0.001 & 0.005 & 0.004 & 0.006 & 0.006 & 0.005 \\
\hline Octanal & 0.005 & 0.010 & 0.002 & 0.007 & 0.003 & 0.006 \\
\hline Nonanal & 0.009 & 0.027 & 0.015 & 0.010 & 0.052 & 0.024 \\
\hline Decanal & 0.008 & 0.017 & 0.001 & 0.006 & 0.003 & 0.007 \\
\hline Total & 0.046 & 0.082 & 0.092 & 0.082 & 0.182 & 0.056 \\
\hline Compound & $\begin{array}{l}\text { Laund. } \\
\text { Cotton }\end{array}$ & $\begin{array}{l}\text { Soiled } \\
\text { Cotton } \\
\end{array}$ & $\begin{array}{c}\text { Laund. } \\
\text { Wool }\end{array}$ & $\begin{array}{c}\text { Soiled } \\
\text { Wool } \\
\end{array}$ & $\begin{array}{c}\text { Laund. } \\
\text { Polyester } \\
\end{array}$ & $\begin{array}{c}\text { Soiled } \\
\text { Polyester } \\
\end{array}$ \\
\hline Formaldehyde & 0.023 & 0.000 & 0.003 & 0.014 & 0.011 & 0.000 \\
\hline Acetaldehyde & 0.010 & 0.006 & 0.002 & 0.003 & 0.002 & 0.002 \\
\hline Acetone & 0.033 & 0.139 & 0.041 & 0.162 & 0.048 & 0.151 \\
\hline Pentanal & 0.003 & 0.000 & 0.001 & 0.000 & 0.003 & 0.000 \\
\hline Hexanal & 0.009 & 0.002 & 0.003 & 0.002 & 0.007 & 0.001 \\
\hline Heptanal & 0.005 & 0.001 & 0.004 & 0.002 & 0.004 & 0.000 \\
\hline Octanal & 0.004 & 0.005 & 0.003 & 0.003 & 0.008 & 0.002 \\
\hline Nonanal & 0.018 & 0.012 & 0.014 & 0.012 & 0.017 & 0.006 \\
\hline Decanal & 0.008 & 0.014 & 0.004 & 0.006 & 0.009 & 0.008 \\
\hline Total & 0.113 & 0.180 & 0.075 & 0.206 & 0.109 & 0.170 \\
\hline
\end{tabular}


Table 3C-3: Surface category-averaged deposition velocity, yield, and surface-to-volume ratio (upper table) and calculated contributions of each surface to area, ozone consumption, and byproduct emissions (lower table).

Surface category-averaged parameters

\begin{tabular}{cccccc}
\hline Material & $\mathrm{v}_{\mathrm{d}}\left(\mathrm{cm} \mathrm{s}^{-1}\right)$ & $\mathrm{Y}(-)$ & $\mathrm{S} / \mathrm{V}\left(\mathrm{m}^{-1}\right)$ & $\begin{array}{c}\mathrm{S} / \mathrm{V} \times \mathrm{v}_{\mathrm{d}} \\
\left(\mathrm{h}^{-1}\right)\end{array}$ & $\begin{array}{c}\mathrm{S} / \mathrm{V} \times \mathrm{v}_{\mathrm{d}} \times \mathrm{Y} \\
\left(\mathrm{h}^{-1}\right)\end{array}$ \\
\hline \hline Plastic & 0.121 & 0.12 & 2.49 & 10.9 & 1.3 \\
Carpet & 0.332 & 0.10 & 0.73 & 8.7 & 0.9 \\
Seat Fabric & 0.379 & 0.13 & 0.91 & 12.4 & 1.7 \\
Clothing & 0.294 & 0.16 & 1.82 & 19.3 & 3.1 \\
\hline Total & & & 5.95 & 51.3 & 6.9
\end{tabular}

Surface contributions to area, ozone consumption and byproduct emissions (percentage of total)

\begin{tabular}{cccc}
\hline Material & $\mathrm{S} / \mathrm{V}$ & $\mathrm{S} / \mathrm{V} \times \mathrm{v}_{\mathrm{d}}$ & $\mathrm{S} / \mathrm{V} \times \mathrm{v}_{\mathrm{d}} \times \mathrm{Y}$ \\
\hline \hline Plastic & $42 \%$ & $21 \%$ & $18 \%$ \\
Carpet & $12 \%$ & $17 \%$ & $13 \%$ \\
Seat Fabric & $15 \%$ & $24 \%$ & $24 \%$ \\
Clothing & $31 \%$ & $38 \%$ & $45 \%$ \\
\hline
\end{tabular}

${ }^{*}$ The data presented this table are plotted in Figure 3-12. 

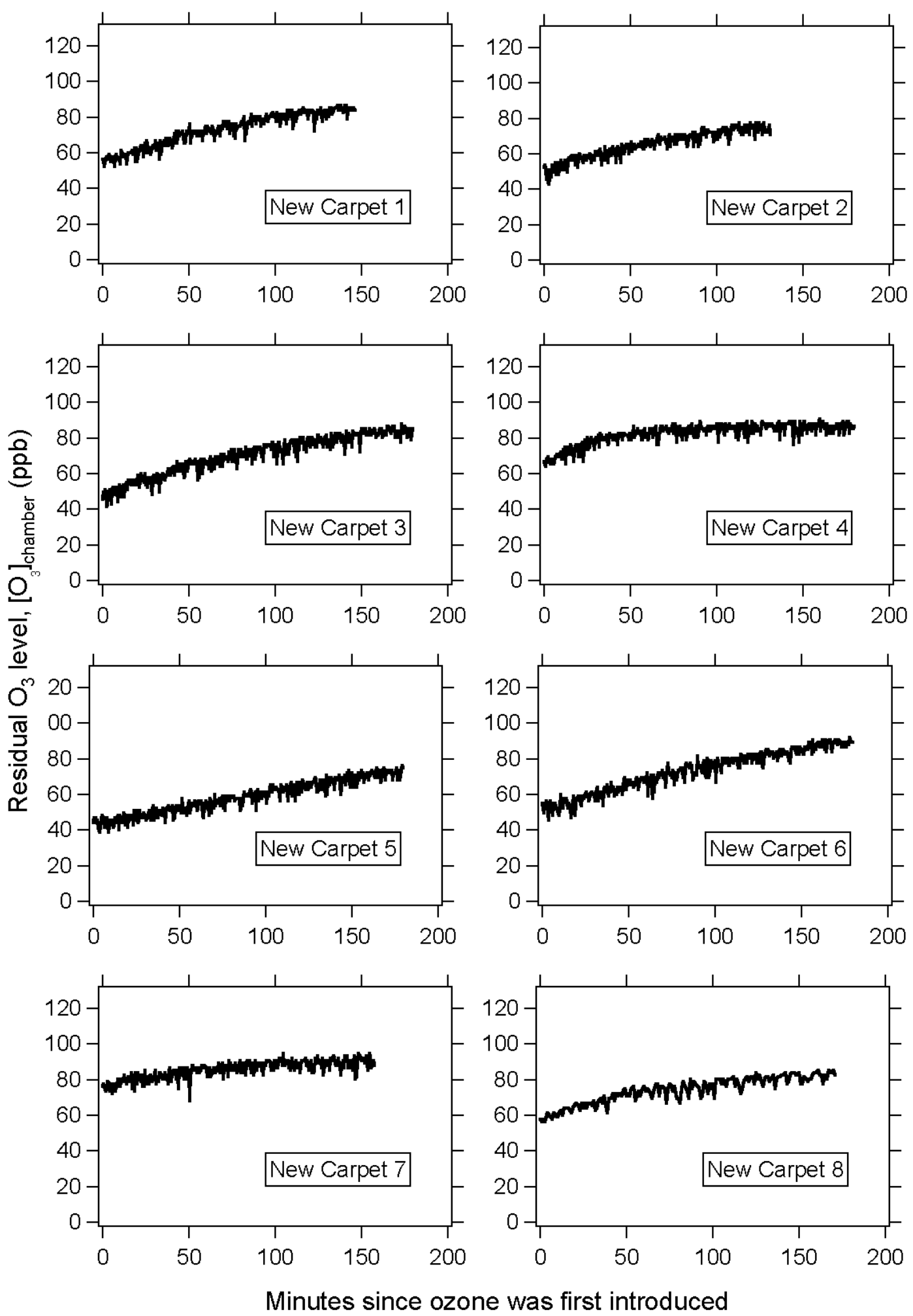

Figure 3C-1a: Ozone data for deposition experiments with new carpet. The supply ozone level was $105 \pm 5 \mathrm{ppb}$. 


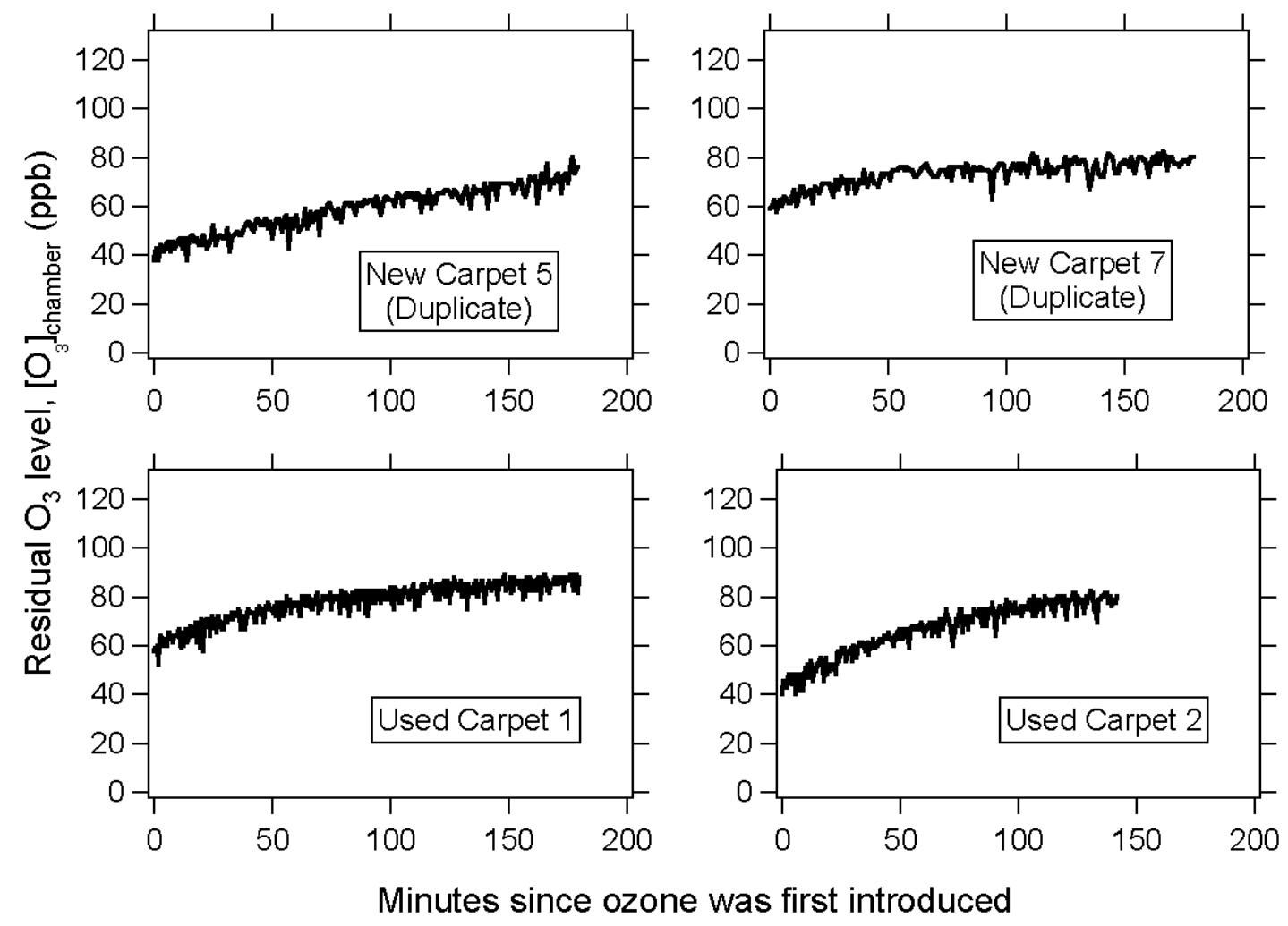

Figure 3C-1b: Ozone data for deposition experiments with new and used carpet. The supply ozone level was $105 \pm 5 \mathrm{ppb}$. 

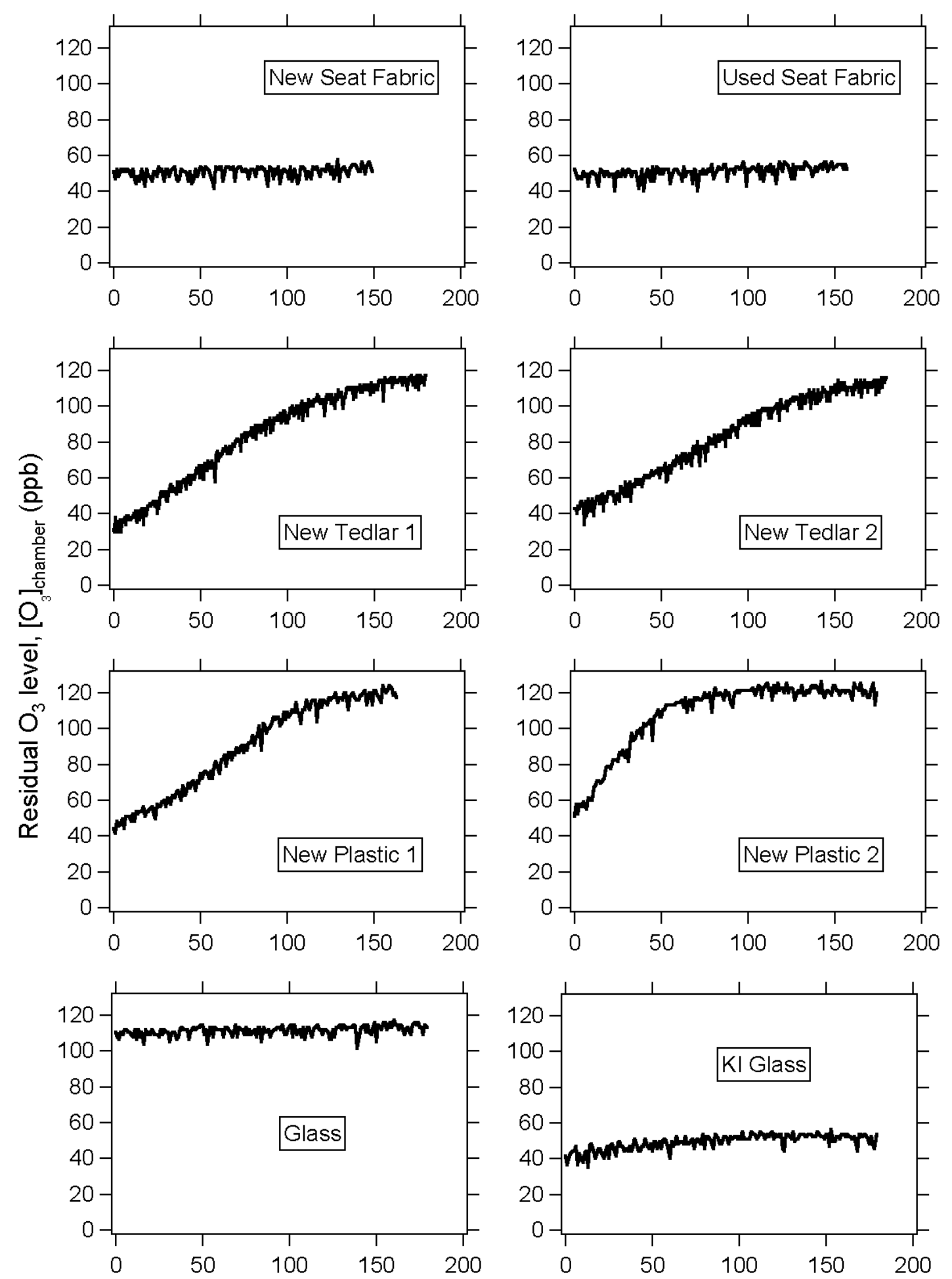

Minutes since ozone was first introduced

Figure 3C-1c: Ozone data for deposition experiments with new and used seat fabric, new plastics, and clean and KI-coated glass. The supply ozone level was $120 \pm 5 \mathrm{ppb}$. 


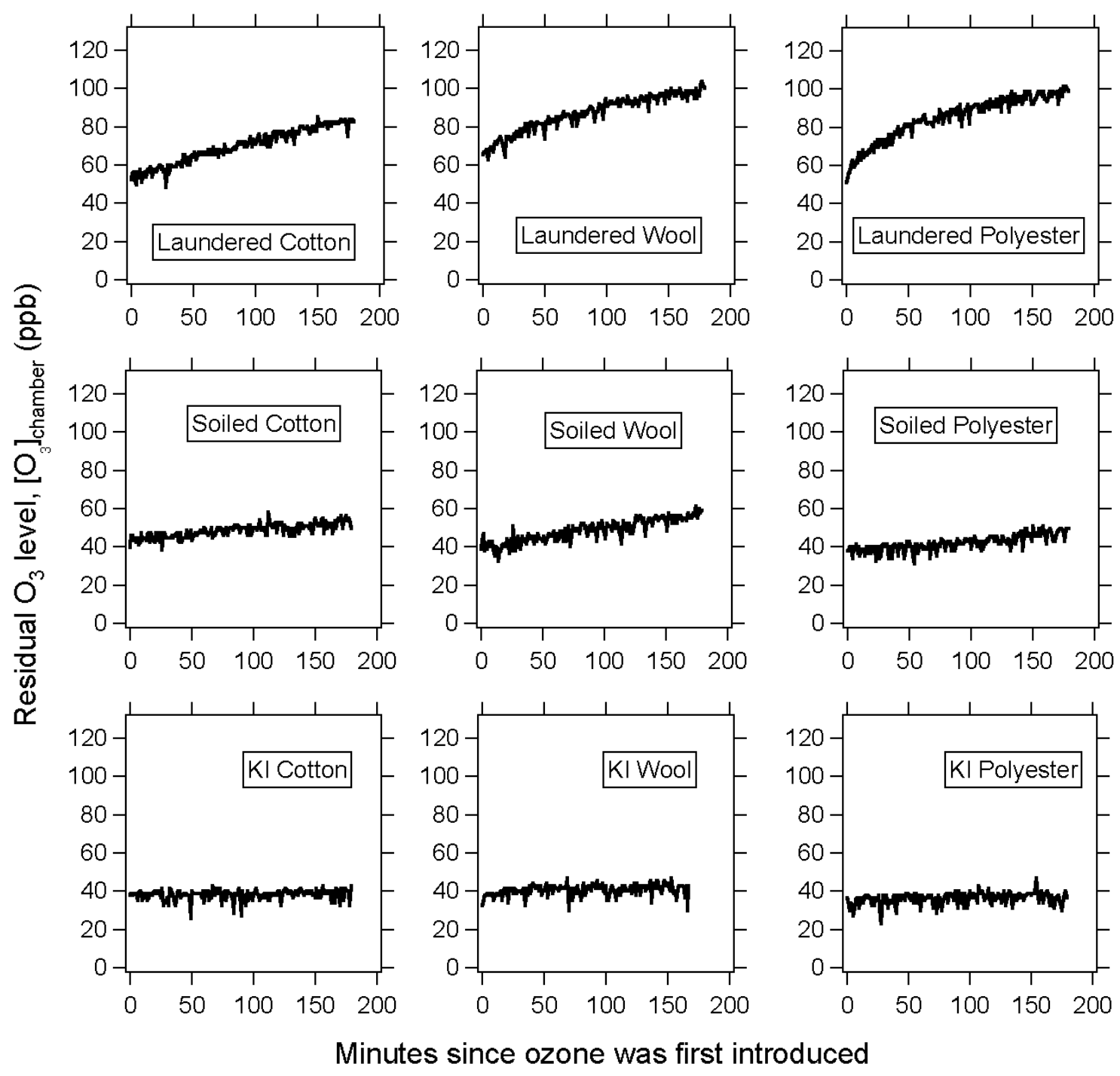

Figure 3C-1d: Ozone data for deposition experiments with laundered, soiled, and KIcoated clothing fabrics. The supply ozone level was $120 \pm 5 \mathrm{ppb}$. 


\section{Ozone reactions with, and byproduct formation from, residual chemicals on indoor surfaces}

\subsection{Introduction}

In indoor environments, ozone reactions with surfaces are the dominant sink of ozone and an important source of volatile oxidation byproducts (Weschler, 2000). Ozone reacts with unsaturated (double) carbon bonds, so any surface chemical product that contains a carbon-carbon double bond (an unsaturation) has the potential to be ozonereactive. Surface materials may be inherently reactive, reactive compounds may be applied during the manufacturing of the product, or reactive compounds may be applied or accrete on surfaces during use. Ozone-reactive surfaces and residues will reduce indoor ozone levels but will also contribute to the generation of oxidation byproducts.

Chemicals that are commonly applied to surfaces during manufacture or use include cleaning products, finishing agents, pesticides, and flame retardants; some of these have been confirmed to contain reactive constituents that remain on the surface. Reactive residues typically belong to two classes of chemicals, terpenes and fatty acids. In one study of ozone reactions with cleaning products, a pine-oil general-purpose cleaner, an orange-oil-based degreaser, and the liquid contents of a heated air freshener were applied to glass plates and aired until gas-phase emissions were below detection. The terpene-based residues exhibited significant reactivity with ozone and produced secondary organic aerosol (Destaillats et al., 2006a). Gas-phase oxidation byproducts have also been detected from an isolated constituent of pine-oil cleaner, $\alpha$-terpineol, in 
experiments where the terpene was applied to glass and vinyl tiles (Ham and Wells, 2008).

Morrison and Nazaroff (2002a) found that carpet emissions in the presence of ozone were similar to emissions from ozone reactions with surface films of linseed oil and tung oil, which contain fatty acids. They proposed that fatty acids on carpets are residues from the manufacturing process. Wang and Morrison (2006) found that ozone byproduct emissions from kitchen counters were consistent with the predicted oxidation byproducts of oleic and linoleic acids, which are common constituents of cooking oils. "Low VOC paints" may contain linseed oil, which includes linoleic and linolenic acids; these paints have been found to produce saturated aldehydes and carboxylic acids when exposed to ozone (research reported in Swedish by Anderson et al., 1996 as described in English by Weschler, 2006). Several recent studies have found that skin-oil residue is readily transferred to clothing fabric and is highly reactive with ozone because skin oil contains significant amounts of squalene, a triterpene, and unsaturated fatty acids or triglycerides (three fatty acids joined by a glycerol molecule) (Chapter 3 of this dissertation; Wisthaler et al., 2005; Tamás et al., 2006). Wells et al. (2008) demonstrated ozone uptake by, and byproduct formation from, squalene and oleic acid applied to a glass plate.

From these studies of ozone-surface chemistry it is clear that chemicals may be applied or otherwise accumulate that significantly change the reactivity and byproduct emissions from a surface. Ozone-surface reactions have been modeled in a macroscopic way that conceptualizes surfaces as homogeneous and flat (Cano-Ruiz et al., 1993). In addition, at least two modeling studies have investigated ozone uptake through a complex 
material matrix, including carpet pile (Morrison and Nazaroff, 2002b) and fiberglass insulation (Liu and Nazaroff, 2001). However, ozone reaction on a surface with heterogeneous composition has not been modeled.

In this chapter, a model is presented for quantifying ozone uptake by a surface partially covered with a reactive residue. The model is used to predict the time required for ozone reactions to consume the residue and to estimate the resulting byproduct emission profile. Use of the model is illustrated with an example of a relatively nonreactive surface that is initially partially covered in oleic acid, which has moderate reactivity, and a surface partially covered in more highly reactive compound, such as a terpene. The effects of several factors - residue reactivity, base material reactivity, surface coverage, airflow conditions, and ozone level - on model outputs such as ozone deposition loss rate, residue consumption, and byproduct formation are explored. The model is also applied to measurements from chamber experiments.

\subsection{Ozone uptake by residual compound}

In this section, ozone transport and uptake is explained conceptually, the mathematical framework is outlined, and the assumptions and limitations of this model are discussed.

\subsubsection{Conceptual illustration and model scope}

Consider a surface that is exposed to well-mixed room air, such as a kitchen counter (see Figure 4-1). The surface is assumed to be smooth and nonporous. The surface, as well as the residue, may be set to have any level of reactivity. To this surface, some amount of a chemical is applied that covers a fraction of the surface, for instance, 
$50 \%$ of the nominal surface area, or all of the surface area in several monolayers.

Consumer products designed for surface application commonly have some constituents that are very volatile, and which tend to evaporate, and some constituents that are less volatile or non-volatile, which will stay on the surface. Examining ozone reactions with a nonvolatile residual constituent is the focus of this analysis.

In the model, I assume that the residue molecules are evenly distributed on the surface in a "sparse monolayer." In actuality, even when fewer molecules are applied than are necessary to form complete monolayer coverage, the molecules of interest may "clump" on the surface in both the vertical and horizontal dimensions. However, in this first attempt to model ozone reactions on a partially covered surface, the coverage is assumed to be even with no more than one molecule "stacked" in the vertical direction. Alternatively and equivalently, one can assume that the spatial distribution of the residual molecules does not affect their reactivity with ozone.

In this chapter, residue molecules that have only one carbon-carbon double bond are considered, and thus it can be assumed that the primary ozone-residue reaction byproducts are not also reactive with ozone. I will make a further simplification by not considering the formation or reaction of the hydroxyl radical $(\mathrm{OH})$, which is typically formed from the reaction of ozone with alkenes in the gas phase (Chew and Atkinson, 1996). Hydroxyl radical formation has been measured under indoor conditions for ozoneinitiated reactions with gaseous terpenes (Fan et al., 2003; Destaillats et al., 2006a). The hydroxyl radical is a powerful and less selective oxidizer than ozone; it reacts rapidly with not only double bonds, but also less electron-rich bonds. Hydroxyl radical formation from ozone reactions with surface-bound compounds has yet to be measured. Lacking 
empirical evidence with which to ground the model, the formation of $\mathrm{OH}$ and its consequences is not considered here.

The temperature of the surface may affect volatilization of the residue and possibly the chemical reaction rate on the surface. The effects of temperature are not considered in this model but could be important for real indoor surface ozone uptake and byproduct emissions.

Ozone uptake begins with transport from bulk air in the room through a concentration boundary layer to the surface, where it reacts with the residual chemical. The residual chemical is consumed and byproducts of the reaction are formed, which either volatize or remain on the surface.

\subsubsection{Mathematical approach}

Deposition velocity is useful for parameterizing removal of ozone by surfaces, and indoor environments are commonly represented as well-mixed continuous flow reactors to calculate ozone removal using deposition velocity. For the case of a wellmixed space that is ventilated with ozone-containing outdoor air, the mass balance on ozone in the space is given by equation 4-1.

$$
\frac{d\left[\mathrm{O}_{3}\right]_{\text {indoor }}}{d t}=\lambda\left[\mathrm{O}_{3}\right]_{\text {outdoor }}-\lambda\left[\mathrm{O}_{3}\right]_{\text {indoor }}-\sum_{i} v_{d, i} \frac{S_{i}}{V}\left[\mathrm{O}_{3}\right]_{\text {indoor }}
$$

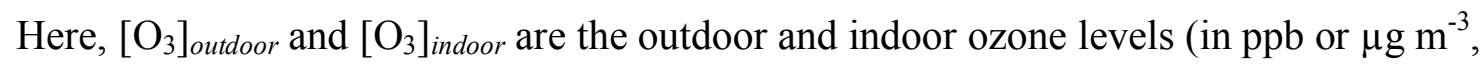
for example), respectively. The space has a volume of $V$ and is ventilated with an airexchange rate of $\lambda$. The index $i$ corresponds to the different types of surfaces with area $S_{i}$ that have an ozone deposition velocity, $v_{d, i}$. The term $v_{d} \times S / V$ (the deposition velocity multiplied by surface-to-volume ratio) defines a first order rate constant for ozone 
removal by a surface; the analogous term in the gas phase is $k_{2} \times C_{\text {reactant }}$ (the second order reaction rate constant for ozone and the reactant multiplied by reactant concentration). The mass balance on ozone presented in equation 4-1 neglects the loss of ozone as air enters the building. Such losses may occur along infiltration pathways (Liu and Nazaroff, 2001) or by incidental or deliberate filtration in mechanical supply airflow (Morrison et al., 1998).

Several studies of ozone removal in actual indoor spaces have validated that ozone removal by surfaces is a first-order process. Lee et al. (1999) measured ozone deposition in 43 homes and found an average first-order removal rate of $2.8 \pm 1.3 \mathrm{~h}^{-1}$. Mueller et al. (1973) measured ozone deposition in a bedroom and an office and found somewhat higher rates of 7.0 and $4.0 \mathrm{~h}^{-1}$. Sabersky et al. (1973) found that the ozone removal rate varied from 2.9 to $5.4 \mathrm{~h}^{-1}$ when forced air was used as opposed to no forced air. A summary of ozone removal rate measurements can be found in Weschler (2000).

In the predominant model of pollutant deposition to surfaces, the core of an indoor space is considered to be well mixed with a thin pollutant-concentration boundary layer separating surfaces from the core pollutant concentration (Figure 4-2a). Nazaroff and Cass (1989) discuss the conceptualization of indoor deposition velocity as the rate of pollutant transport through the near-surface concentration boundary layer. In the work of Nazaroff and Cass, model equations were developed for gas and particle deposition under transport-limited conditions, i.e. for cases when the pollutant of interest is taken up by the surface as rapidly as transport processes can replenish the pollutant concentration near the surface. Cano-Ruiz et al. (1993) extended that model to incorporate the kinetics of pollutant uptake at the surface for reactive gases. 
In the model of Cano-Ruiz et al. (1993), ozone deposition velocity captures two processes, transport through the boundary layer and transformation (sorption or reaction) at the surface. If the pollutant is very rapidly transformed at the surface, then the deposition velocity is termed transport-limited. If the transformation is not rapid, e.g. the airborne pollutant is not very reactive with the surface, then pollutant deposition is termed kinetically-limited. Cano-Ruiz et al. (1993) used a mathematical framework similar to that used to calculate deposition velocities to outdoor surfaces. They conceptualized deposition as the inverse sum of two resistances in series, where one resistor represents transport and the other represents uptake at the surface. This concept is illustrated in Figure 4-2b. The result of this mathematical framework is an equation for deposition velocity that accounts for both processes (equation 4-2).

$$
v_{d}=\left[r_{\text {tranport }}+r_{\text {uptake }}\right]^{-1}=\left[\frac{1}{v_{t}}+\frac{1}{v_{s}}\right]^{-1}
$$

The terms $1 / v_{t}$ and $1 / v_{s}$ represent the transport resistance and uptake resistance, respectively. Here, $v_{t}$ is the transport-limited deposition velocity, i.e. the maximum deposition velocity that would be observed for a given set of conditions if the surface was optimally reactive with the pollutant.

The surface uptake velocity, $v_{s}$, is shown in equation 4-3 and was derived from the molecular theory of gases. The term $\langle v\rangle / 4$ is the effective average speed normal to the surface at which molecules collide with the surface. The Boltzmann velocity, $\langle v\rangle$, is $3.6 \times 10^{4} \mathrm{~cm} \mathrm{~s}^{-1}$ for ozone at $293 \mathrm{~K}$. This term is multiplied by the reaction probability, $\gamma$, to get the rate of collisions that result in uptake. 


$$
v_{s} \approx \gamma \frac{\langle v\rangle}{4}
$$

Reaction probability is the parameter that describes the efficiency of uptake at the surface. Reaction probability is defined as the fraction of all pollutant molecule collisions with the surface that result in irreversible removal (Cano-Ruiz et al., 1993), or the rate of species uptake divided by the rate at which the species strikes the surface (Morrison and Nazaroff, 2002b). Reaction probability is also called "uptake coefficient" (Utter et al., 1992), "sticking coefficient” (Stephens et al., 1986), or “net collisional uptake probability" (Finlayson-Pitts and Pitts, 2000).

In the Cano-Ruiz et al. (1993) model of ozone transport and uptake, the masstransport-limited deposition velocity for turbulent flow was estimated using analytical models of eddy diffusivity. Later, Lai and Nazaroff (2000) developed mathematical expressions to describe turbulent diffusion of particles near surfaces based on the direct numerical simulations of Kim et al. (1987). Morrison and Nazaroff (2002b) extended the model of Lai and Nazaroff (2000), which was developed for particles, to describe nearsurface turbulent diffusion of gas molecules. The relevant difference between a gas molecule and a particle for this model is the species size and its consequent molecular diffusivity (or Brownian diffusivity, in the case of particles). The size of a molecule is on the order of $1 \mathrm{~nm}$, and particles for which diffusion matters ranges from a cluster of molecules to $\sim 0.3 \mu \mathrm{m}$. Building on the work of Lai and Nazaroff (2000), Morrison and Nazaroff (2002b) determined that the transport-limited deposition velocity could be modeled as the ratio of $u^{*}$ to $\Gamma$.

$$
v_{t} \approx \frac{u^{*}}{\Gamma}
$$


The parameter $\Gamma$ is a nondimensional factor that results from integrating the ratio of kinematic viscosity to the overall species diffusivity (molecular and turbulent). For ozone under typical indoor conditions, $\Gamma$ was determined to be equal to 13.3 by numerical integration of an expression describing molecular and turbulent diffusion flux through the concentration boundary layer (Morrison and Nazaroff, 2002b). The friction velocity, $u^{*}$, is an indication of near-surface flow conditions related to turbulence intensity and has units of $\mathrm{cm} \mathrm{s}^{-1}$.

Substituting the equations for transport-limited and surface-uptake velocities (equations 4-3 and 4-4) into the equation for deposition velocity derived from resistor theory (equation 4-2) gives the following expression describing reactive gas deposition on surfaces (equation 4-5) (Morrison and Nazaroff, 2002b).

$$
v_{d}=\frac{\gamma\langle v\rangle u^{*}}{\Gamma \gamma\langle v\rangle+4 u^{*}}
$$

This equation has been successfully used to describe deposition dynamics in experiments of ozone chemistry (Chapter 3 of this dissertation).

Ozone removal by individual surfaces appears to be reasonably well described by the expression presented in equation 4-5, and overall ozone deposition appears to be first order in indoor environments (as described by equation 4-1). Thus, it is reasonable to combine these equations to investigate ozone uptake in indoor spaces. Furthermore, it is reasonable to extend this model to explain ozone reactions with surfaces that are heterogeneous. In this chapter, I build on the framework of ozone transport and uptake to develop a model of ozone reactions with, and byproduct formation from, an ozonereactive residue. The major application of this model is in simulating ozone deposition to 
a surface that comprises a stable base material plus a consumable residue. In this framework, the ozone concentration boundary layer is modeled as a three-resistor model, with one resistor representing transport through the boundary layer, and two parallel resistors representing ozone uptake at the surface by the residue and the base material, as shown in Figure 4-2b.

For the case of a surface with a residual chemical, the overall reaction probability, $\gamma$, is equal to the weighted sum of the reaction probabilities for the base material and the residue (equation 4-6), where $r$ is the fraction of the nominal surface area that is covered in residue, and $\gamma_{X}$ and $\gamma_{B}$ are the reaction probabilities of the residue and the base, respectively.

$$
\gamma=r \gamma_{X}+(1-r) \gamma_{B}
$$

Figure 4-3 shows the effect on overall ozone deposition $\left(v_{d}\right)$ of progressively adding more reactive residual chemical to a surface with a base reaction probability of $\gamma_{\mathrm{B}}$ $=10^{-5}$. The "null case" is base material with no residue $(r=0)$; the overall deposition to the surface does not depend on the reactivity of the residue, and thus the line is flat. At the other extreme, deposition to a surface fully covered in the residue $(r=1)$ would only depend on the residue's reaction probability; note that this trace has the same shape as the curves shown in Figure 3-4. The other cases show how deposition velocity is affected as the surface is $1 \%, 10 \%$, and $50 \%$ covered in a residue $(r=0.01,0.1,0.5$, respectively) for residue reaction probabilities $\gamma_{X}=10^{-7}-10^{-1}$. Each curve plateaus at the same value of $v_{d}$ at the higher values of $\gamma_{X}$, because deposition velocity is limited by mass transport to the surface when the reactivity of the surface is very high. The maximum deposition velocity depends on flow conditions, which is captured by the parameter friction velocity $\left(u^{*}\right)$ in 
this model. For low reactivity residues, there is little effect of residue surface coverage on the overall surface reactivity for low to moderate degrees of coverage. For example, the $r$ $=0.5$ curve lies very near the $r=0$ curve for $\gamma_{X}<<\gamma_{B}$. However, for high reactivity residues, the residue significantly affects deposition to the surface, and the extent of coverage does not strongly influence the outcome for moderate to high fractional coverages. For instance, at $50 \%$ coverage $(r=0.5)$, deposition velocity is comparable to the $r=1$ case.

Figure 4-3 also contains information about the way that ozone deposition to the surface, and thus ozone reactions and byproduct formation from the residue, might evolve over time as the residue is consumed in reactions with ozone. For a high reactivity residue, deposition velocity would decrease from its original value corresponding to the initial surface coverage, e.g. $r=0.1$, to $r=0$ as the residue is consumed. In the following section, equations are developed that define the time-dependent ozone reactivity and predict byproduct emission profiles.

\subsection{Time-dependent model of deposition and byproduct formation: Mathematical description of model}

The basis of this model is a well-mixed indoor space where ozone reactions on surfaces are the dominant sink for ozone. A mass balance on ozone in the space as given by equation 4-1 is repeated here for the reader's convenience.

$$
\frac{d\left[\mathrm{O}_{3}\right]_{\text {indoor }}}{d t}=\lambda\left[\mathrm{O}_{3}\right]_{\text {outdoor }}-\lambda\left[\mathrm{O}_{3}\right]_{\text {indoor }}-\sum_{i} v_{d, i} \frac{S_{i}}{V}\left[\mathrm{O}_{3}\right]_{\text {indoor }}
$$

Note that when considering chamber experiments, subscripts "supply" and "chamber" could be substituted for "outdoor" and "indoor," respectively. For the remainder of the 
chapter the subscript "indoor" will be dropped when discussing the indoor or chamber ozone concentration.

In this model, the molecular surface coverage of the residue is calculated at each time step by subtracting the amount of residue consumed in the previous step, which is dependent on ozone deposition. An explanation of the model and important parameters follows.

Molecular footprint, $A_{m o l e c}\left(\mathrm{~m}^{2}\right.$ molecule $\left.{ }^{-1}\right)$. The model requires input of the physical characteristics of the residue - molecular weight $\left(\mathrm{g} \mathrm{mol}^{-1}\right)$ and liquid density ( $\mathrm{g}$ $\mathrm{cm}^{-3}$ ) - to determine the approximate molecular surface coverage of the residue (molecule or mole per area). The "footprint" of a residue molecule is estimated by assuming that each molecule occupies a cubic volume equal to its molecular weight divided by its density and Avogadro's number. The molecular volume is raised to the two-thirds power to get the characteristic area covered by the molecule. This is a rough estimate of molecular area because molecules, especially the larger variety that would tend to partition to a surface, may have more complex geometry.

Monolayer surface coverage, $m$, and fractional surface coverage, $r$. In the model, the surface may be partially covered, or may be completely covered in monolayers of residue. The initial surface coverage is an input parameter of the model, $m(0)$. Surface coverage is defined as the number of monolayers covering of actual surface area, and may be a fraction value. For example, $m(t)=2$ indicates a surface of two monolayers, and $m(t)=0.1$ indicates a surface coverage of $10 \%$. After the initial time step, coverage at time $t, m(t)$, is calculated by multiplying the initial surface coverage, 
$m(0)$, by the ratio of residue concentration at time $t, R(t)$, to the initial residue concentration, $R(0)$, as expressed in equation 4-7.

$$
m(t)=m(0) \frac{R(t)}{R(0)}
$$

The monolayer coverage parameter, $m$, is useful for keeping track of the absolute surface coverage. The fractional surface coverage parameter, $r$, is useful in other calculations when just the fraction of the surface coverage is important. At time $t, r(t)$, is equal to 1 if a monolayer of residue or more is present. Otherwise, $r(t)$ is equal to $m(t)$. This relationship between the two coverage parameters is expressed in equation 4-8.

$$
\begin{aligned}
& r(t)=1, \text { if } m(t) \geq 1 \\
& r(t)=m(t), \text { if } m(t)<1
\end{aligned}
$$

Residue surface concentration, $R\left(\mathrm{~mol} \mathrm{~m}^{-2}\right)$. Initially the residue surface concentration, $R(0)$, is calculated by dividing the initial fractional surface coverage, $r(0)$, by the area of a molecule, $A_{\text {molec }}\left(\mathrm{m}^{2}\right.$ molecule $\left.{ }^{-1}\right)$ and Avogadro's number, $A_{v}$ (molecule $\left.\mathrm{mol}^{-1}\right)$, as shown in equation 4-9. Here it is helpful to think of $r(0)$ as having units of area of residue per area of surface.

$$
R(0)=\phi \frac{r(0)}{A_{\text {molec }} A_{v}}
$$

After the initial time step, residue surface concentration is calculated by solving the differential equation describing the rate of residue consumption as shown in equation 4-10. Equation 4-10b is the critical calculation step that progresses the model forward. The updated value of $R(t)$ is used to compute new values of $m(t)$ by equation 4-7 and $r(t)$ by equation $4-8$ at each iteration. 


$$
\begin{aligned}
& \frac{d R}{d t}=-\left[\mathrm{O}_{3}\right] f v_{d} \approx \frac{\Delta R}{\Delta t} \\
& R(t+1)=R(t)-\left[\mathrm{O}_{3}\right](t) f(t) v_{d}(t) \Delta t
\end{aligned}
$$

The parameter $\Delta t$ is the time-step in seconds, and the other parameters $\phi,\left[\mathrm{O}_{3}\right], f(t)$, and $v_{d}(t)$ are discussed in the following paragraphs.

Surface area multiplication factor, $\phi$. Many surfaces materials have a much greater actual surface area than nominal surface area. This concept is illustrated in Figure 4-4. For example, Morrison and Nazaroff (2002b) estimated that the carpet samples in their study had a surface area multiplication factor (they referred to this parameter as normalized surface area) of 30-66. The importance of increased surface area is that there are many more "sites" for ozone reaction with the surface per nominal surface area. The relationship between nominal surface area, $S$, and estimated actual surface area, $S_{A}$, is shown in equation 4-11.

$$
S_{A}=\phi S
$$

The effect of increasing $\phi$ in this model is to increase the initial amount of residue per nominal surface area (see equation 4-9) and as a result, increase the time required for ozone to consume the residue. For example, a flat surface with $10 \%$ coverage by a fatty acid might have and initial residue concentration of $\sim 2.5 \times 10^{-7}$ mole $\mathrm{m}^{-2}$. A textured surface with $30 \times$ greater actual area than nominal surface area $(\phi=30)$ that has $10 \%$ coverage with a fatty acid will have $\sim 7.5 \times 10^{-6}$ mole per $\mathrm{m}^{2}$ of nominal surface area. The initial fraction of the surface covered by residue is defined by $m(0)$ and is not affected by an increase in $\phi$. At subsequent time steps, the rate of decrease in $m$ is lower for the surface with higher $\phi$. 
The increased surface area on the micro-scale level is not expected to affect transport of ozone through the boundary layer; thus, $\phi$ is assumed to not affect the masstransport resistance. Increased surface area for a given surface reaction probability may decrease the surface uptake resistance. However, this model is formulated so that increased surface area does not have a direct effect on the surface resistance. Here, the affect of uptake is expressed as a reaction probability $(\gamma)$. Since the surface coverage level is set as a model input parameter, the probability of an ozone molecule reacting with a surface-bound molecule is assumed to not change with increased surface area. In addition, the surface area over which ozone deposits, $S$, was not multiplied by $\phi$ because amount of residue on the surface $(R(t))$ already takes into account the effect of increased surface area.

Ozone concentration, $\left[\mathbf{O}_{3}\right]\left(\mathrm{mol} \mathrm{m}^{-3}\right)$. In this chapter, two methods of handling ozone were used. The first method was to hold the indoor or chamber ozone concentration constant. The second method was to allow the chamber or indoor ozone level to vary dynamically using coupled differential equations for ozone and residue concentrations.

The first method - holding the indoor ozone concentration constant - is most appropriate for a setting where the indoor ozone level is not significantly affected by ozone consumption by the residue on the surface of interest. This would be the case in a space where there are many ozone-consuming surfaces but only one ozone-reactive residue-covered surface of interest. The mass-balance on ozone (equation 4-1) is rewritten in equation 4-12 with separate ozone deposition terms for the surface of interest and all other surfaces. 


$$
\frac{d\left[\mathrm{O}_{3}\right]}{d t}=\lambda\left[\mathrm{O}_{3}\right]_{\text {outdoor }}-\lambda\left[\mathrm{O}_{3}\right]-\sum_{i} v_{d, i} \frac{S_{i}}{V}\left[\mathrm{O}_{3}\right]-v_{d, \text { interest }} \frac{S_{\text {interest }}}{V}\left[\mathrm{O}_{3}\right]
$$

If ozone uptake by the residue-covered surface $\left(v_{d, \text { interest }} \times S_{\text {interest }} / V\right)$ is small compared with the two other loss terms (ventilation and deposition to all other surfaces), then its effect on time-dependent ozone levels will also be small.

The second method - allowing ozone level to vary with time - is potentially useful for modeling ozone deposition in chamber experiments where, for example, one surface is exposed to ozone and the chamber ozone level is used to determine deposition velocity, reaction probability, or both. When ozone concentration is allowed to vary, the model ozone concentration at each time step is determined using equation 4-13, which was derived from the material balance on ozone (equation 4-1).

$$
\left[\mathrm{O}_{3}\right](t+1)=\left[\mathrm{O}_{3}\right](t)+\Delta t\left(\lambda\left[\mathrm{O}_{3}\right]_{\text {supply }}-\lambda\left[\mathrm{O}_{3}\right](t)-v_{d}(t) \frac{S}{V}\left[\mathrm{O}_{3}\right](t)\right)
$$

The indoor ozone level is entered in units of $\mathrm{ppb}$ and is converted to units of mole per $\mathrm{m}^{3}$ for use in the model. The model assumes a temperature of $295 \mathrm{~K}\left(72{ }^{\circ} \mathrm{F}\right)$ and a pressure of $1 \mathrm{~atm}$.

\section{Overall reaction probability, $\gamma$, and deposition velocity, $v_{d}\left(\mathrm{~cm} \mathrm{~s}^{-1}\right)$. The} overall reaction probability of the surface, including base and residue, is calculated at each time step using equation 4-6, rewritten below with the time-dependent parameters indicated.

$$
\gamma(t)=r(t) \gamma_{X}+(1-r(t)) \gamma_{B}
$$

This parameter can then be used to calculate the overall deposition to the surface using equation 4-1, which is shown again here with the time dependent variables indicated. 
Deposition velocity must be converted to units of $\mathrm{m} \mathrm{s}^{-1}$ before use in equations $4-10 \mathrm{~b}$ and 4-15.

$$
v_{d}(t)=\frac{\gamma(t)\langle v\rangle u^{*}}{\Gamma \gamma(t)\langle v\rangle+4 u^{*}}
$$

Residue-ozone fraction, $f$. To determine the amount of residue that was consumed, the total ozone deposition must be multiplied by the fraction of ozone that reacted with the residue. The ratio of ozone reacting with the residue to total ozone reacting with the surface of interest, $f$, is termed "residue-ozone fraction", and is calculated using equation 4-14. If a monolayer or more is present, then $f(t)=1$ and the deposition velocity is governed by reaction with the residue alone.

$$
f(t)=\frac{r(t) \gamma_{X}}{\gamma}
$$

Byproduct emissions, $\boldsymbol{E}\left(\mathbf{m o l ~ m} \mathbf{m}^{-2} \mathbf{s}^{-1}\right)$. The byproduct emission rate at time $t$, $E(t)$, is equal to the residual consumption rate multiplied by the byproduct yield, $Y$ (equation 4-15). Here, yield is defined as mole of byproduct emitted per mole of ozone consumed.

$$
E(t)=Y\left[\mathrm{O}_{3}\right](t) f(t) v_{d}(t)
$$

Byproduct level, [byproduct] (ppb). The byproduct level at time $t$, [byproduct] $(t)$, was calculated by assuming that the emissions occurred in a well-mixed room of volume of $V\left(\mathrm{~m}^{3}\right)$, with an air-exchange rate of $\lambda\left(\mathrm{h}^{-1}\right)$, and by assuming that the partially coated surface had a nominal surface area of $S\left(\mathrm{~m}^{2}\right)$. The byproduct is assumed to be chemically inert and nonsorbing and other potential sources of the byproduct are neglected. The byproduct concentration was estimated by assuming steady-state 
conditions in the room (equation 4-16). The air-exchange rate is entered in units of per hour, which is converted to a per-second rate, and gives units of $\mathrm{mol} \mathrm{m}^{-3}$ for byproduct concentration. This is converted to byproduct level (ppb) using a temperature of $295 \mathrm{~K}$ $\left(72{ }^{\circ} \mathrm{F}\right)$ and a pressure of $1 \mathrm{~atm}$.

$$
[\text { byproduct }](t)=\frac{E(t) S}{\lambda V}
$$

\subsection{Parametric investigation of model with constant ozone level}

\subsubsection{Model inputs: Base material and residual characteristics}

Reaction probabilities for common indoor surfaces range from $<10^{-7}$ for inert surfaces such as glass or steel to $>10^{-4}$ for very reactive surfaces such as some carpets and human skin oil (Cano-Ruiz et al., 1993; Wang and Morrison, 2006; Pandrangi and Morrison, 2008; Wells et al., 2008; Chapter 3 of this dissertation). Human skin oil is a great example of a highly reactive residual surface compound that is easily transferred from skin onto other surfaces thereby also making them very reactive (Fruekilde et al., 1998; Wisthaler et al., 2005; Tamás et al., 2006; Chapter 3 of this dissertation). About half of ozone reactions with skin oil are with squalene and about half are with singly or doubly unsaturated fatty acids and other similar compounds (Pandrangi and Morrison, 2008). Since squalene has many double bonds and many reaction products, some of which can participate in secondary chemistry (Fruekilde et al., 1998; Wells et al., 2008), the following example will focus on a simpler molecule, such as a fatty acid. Fatty acids are not only prominent in skin oils, but they are also common constituents in some types of consumer products. For instance, linseed oil is used in paints, linoleum, and floor oils (Morrison and Nazaroff, 2002a; Knudsen et al., 2007). Linseed oil contains linoleic acid, 
an omega- 6 fatty acid with two double bonds. Oxidation byproduct emissions from linseed oil, similar oils, and products containing or coated in these oils have been measured and also have been shown to degrade perceived air quality (Salthammer et al., 1999; Morrison and Nazaroff, 2002a; Weschler, 2006; Knudsen et al., 2007).

The basic structure of a fatty acid is a long aliphatic chain, which usually contains zero, one, or two unsaturations, ending with a carboxylic acid group. In this chapter, reaction of surface-bound oleic acid will be explored. Oleic acid is an omega-9 fatty acid with one unsaturation. (Omega- $n$ indicates that the first (or only) double bond is located $n$ carbon atoms from the aliphatic end of the molecule.) Heterogeneous (surface-bound) ozone-oleic acid chemistry has been widely studied in regards to atmospheric aerosol aging (see Zahardis and Petrucci, 2007, and references therein) where oleic acid is used as a surrogate for atmospheric unsaturated organic acids. In the atmospheric aerosol literature, reaction probability is referred to as the reactive uptake coefficient; it is the same parameter, and it is often determined in a similar manner to reaction probabilities for indoor surfaces. The oleic acid-ozone system has also been investigated in one indoor surface chemistry study aimed at quantifying the reaction probability of skin oil components (Wells et al., 2008). From these studies, the reaction probability of oleic acid is reasonably well known, as are the ozone byproducts. Both reaction probability and byproduct yield have been found to depend on conditions such as temperature, relative humidity, and composition of solution (species in liquid or solid phase) and gas-phase composition; inputs for the model were chosen based on common characteristics of indoor environments. 
The typical range of reaction probabilities measured in atmospheric chemistry experiments, which usually involve liquid oleic acid or pure oleic acid aerosol, is $\sim(0.7-$ $1.0) \times 10^{-3}$ (Zahardis and Petrucci, 2007); the reaction probability could be an order of magnitude lower when a mixture or solid phase is present, as would be the case if oleic acid were applied onto an indoor surface. Moise and Rudich (2002) measured the reaction probability to be $0.5 \times 10^{-4}$ for frozen oleic acid, and $8.3 \times 10^{-4}$ for liquid. The difference between the liquid and frozen experiments was that ozone could only react with oleic acid at the air-solid interface of the frozen sample, and thus surface reactions were isolated. The reaction probability of oleic acid measured for roughly monolayer coverage on glass was $0.5 \times 10^{-4}$ (Wells et al., 2008). Based on this evidence, for the model described here, oleic acid is assumed to have $\gamma_{X}=0.5 \times 10^{-4}$.

Typical ozonation byproducts of oleic acid are nonanal, azelaic acid, nonanoic acid, 9-oxononanoic acid (see Figure 4-5). Ozone attacks oleic acid at its double bond, located nine carbons from either end of the molecule, forming an unstable ozonide. The ozonide rapidly dissociates into two fragments, a carbonyl and a Criegee biradical. Nonanal and a Criegee biradical can be formed, or 9-oxononanoic acid and a different Criegee biradical can be formed (Moise and Rudich, 2002). The Criegee biradical is stabilized to form another oxygenated byproduct, such as azelaic acid or nonanoic acid, respectively. There is also the possibility of a secondary ozonide forming on surfaces; it is only formed in solution (i.e., in oleic acid liquid) when fragments stay in proximity to one another and therefore have the opportunity to recombine (Zahardis and Petrucci, 2007). Nonanal is the only byproduct that has been detected in the gas-phase, and the yield has been quantified from ozone-oleic acid reactions. Other products such as azelaic 
acid are known to stay on the surface rather than be emitted because the presence of acid functional groups greatly increases its polarity and water solubility. Measured nonanal yields (mol of nonanal emitted per mol of ozone consumed) range from $20-50 \%$, depending on the experimental conditions and detection methods (Moise and Rudich, 2002; Thornberry and Abbatt, 2004; Hearn and Smith, 2004; Hung and Ariya, 2005). Nonanal has also been measured in studies of indoor surface chemistry, so it is possible to compare the results of this model to laboratory results. For all these reasons, nonanal will be focused on as the byproduct of interest in this model application. A value of $Y=$ 0.3 was used for byproduct yield in the following example.

\subsubsection{Results and discussion}

Figure 4-6 shows two sets of model results, one where oleic acid is the residue and one with a more reactive residue. The base material was set to have a low reaction probability of $\gamma_{B}=10^{-7}$, to isolate the effect of the residue on ozone chemistry. For the

first case, the residual had a reaction probability of $\gamma_{X}=0.5 \times 10^{-4}$, with an initial surface coverage with oleic acid of $10 \%(r=0.1)$. The figure shows the change with time of the overall surface reactivity, $\gamma$, and the fraction, $f$, of ozone that is reacting with the residue relative to the total reactions with the surface. Figure 4-6a also shows the byproduct formation rate, $E$, and the resulting byproduct concentration, [byproduct], assuming a well-mixed room. All four of the parameters gradually decrease as the residual is consumed in ozone reactions.

For comparison, the model was run with another set of model parameters and the output is shown in Figure 4-6b. These inputs are hypothetical, but might correspond to 
cleaning product residue. They differ from the oleic acid example in that the residue has much lower molecule weight $\left(\mathrm{MW}_{\text {res }}=150 \mathrm{~g} \mathrm{~mol}^{-1}\right.$ versus $\left.292 \mathrm{~g} \mathrm{~mol}^{-1}\right)$, a slightly higher density $\left(\rho_{\text {res }}=1 \mathrm{~g} \mathrm{~cm}^{-3}\right.$ versus $\left.0.89 \mathrm{~g} \mathrm{~cm}^{-3}\right)$, and a significantly higher reactivity $\left(\gamma_{X}=10^{-3}\right.$ versus $\left.0.5 \times 10^{-4}\right)$. The byproduct yield was kept at $Y=0.3$.

The hypothetical cleaning product residue is consumed much more quickly than the oleic acid, $\sim 0.4 \mathrm{~h}$ compared to $\sim 1.7 \mathrm{~h}$. The time-scale for Figure $4-6 \mathrm{a}$ (oleic acid) is $4 \times$ longer than the time-scale for Figure 4-6b (highly reactive residue). Also, the temporal profiles of outputs are different for the residues. For the more reactive cleaning product residue, total surface reactivity is initially dominated by the residue $(f$ is sustained at a value near 1) and then very rapidly decreases once enough of the residue has reacted away. In comparison, $f$ in the oleic acid example only gradually decreases. In addition, there are two inflection points in the [byproduct] and $\gamma$ curves for the very reactive residue example, but only one inflection is seen in the oleic acid example.

Figure 4-7 shows the effect of residue reactivity on the temporal evolution of overall deposition velocity and overall reaction probability. The trends in these two parameters are almost identical. Note the x-axis, representing time, is on a log-scale; plotted on a log-log scale, there are two inflection points in the time-dependent $v_{d}$ and $\gamma$ curves for the lower reaction probabilities, but in linear scale there would be just one inflection point. With decreasing reactivity of the residue, the shape of the curves for $v_{d}$ and $\gamma$ transition from a sharp drop to a gradual reduction. Residue duration increases exponentially with the exponential decrease in residue reactivity.

Figure 4-8 shows overall deposition velocity versus time for base reactivities spanning four orders of magnitude, $\gamma_{B}=\left\{10^{-7}, 10^{-6}, 10^{-5}, 10^{-4}\right\}$, and residue reactivities 
spanning five orders of magnitude, $\gamma_{X}=\left\{10^{-7}, 10^{-6}, 10^{-5}, 10^{-4}, 10^{-3}\right\}$. In these model runs the residue was assumed to have a molecular weight of $150 \mathrm{~g} \mathrm{~mol}^{-1}$ and a density of $1 \mathrm{~g}$ $\mathrm{cm}^{-3}$, the initial residue surface coverage was $50 \%(r(0)=0.5)$, the ozone level was 20 $\mathrm{ppb}$, and the friction velocity was $1 \mathrm{~cm} \mathrm{~s}^{-1}$, which is likely an average value inside a building with moderate ventilation. Two important trends can be gleaned from this series of plots. First, if a residue is less reactive than the base material $\left(\gamma_{X}<\gamma_{B}\right)$, and this residue is effective at prohibiting the reaction with the base material, then the overall deposition velocity to the surface will be suppressed until the residue is consumed. Second, the higher the base material reactivity, the less the reactivity of the residue matters to the overall reactivity of the surface. For example, see the bottom frame of Figure 4-8 $\left(\gamma_{B}=\right.$ $\left.10^{-4}\right)$, where the overall deposition velocity spans a very narrow range, $v_{d}=0.65-0.74 \mathrm{~cm}$ $\mathrm{s}^{-1}$, for three orders of magnitude difference in residue reactivity, $\gamma_{X}=10^{-3}-10^{-6}$.

In Figure 4-9, the effects of various model inputs on one model output, ozone deposition velocity, are investigated. In the first frame, residue surface coverage is increased from $10 \%(r(0)=0.1)$ to three monolayers $(m(0)=3)$. Increasing the surface coverage up to monolayer coverage (i.e., from $r(0)=0.1$ to 1.0 ) had the effect of increasing $v_{d}$ because there is more residue available to be consume ozone. Beyond one monolayer, increased surface coverage sustains the maximum deposition velocity for a longer period of time. In the second frame, the effect of increased surface area is shown. As the model is currently constructed, increased $\phi$ does not change the change the shape of the deposition velocity curve; it delays the eventual decrease in reactivity of the residue-covered surface. In the third frame, the effect of airflow conditions on deposition was investigated. Friction velocities of $0.3,1.0,3.0$, and $10 \mathrm{~cm} \mathrm{~s}^{-1}$ were tested with other 
parameters held constant. Increasing $u^{*}$ increases the transport of ozone through the concentration boundary later, so increasing $u^{*}$ increased the overall deposition velocity. In the bottom frame, a range of typical indoor ozone levels $-5,10,20,50 \mathrm{ppb}-$ was entered. The model results confirm intuition: the higher the ozone level, the faster the residue is consumed, and the faster the deposition velocity to the surface of interest decreases.

For the conditions presented here, the model indicates that the reactive residue is consumed (as indicated by the plateau in deposition velocity at its minimum value) in a period of just minutes to hours. Certainly greater residue coverage, decreased reactivity of the residue, and reduced airflow conditions (i.e. lower friction velocity) would increase the amount of time required for the residue to be fully consumed.

\subsection{Application to chamber measurements with variable ozone level}

Most studies of ozone-surface chemistry involve measuring ozone consumption of a single material or residual in a continuously ventilated chamber with ozone supplied at a constant rate. Under these conditions, the ozone concentration in the chamber can vary with time. To test the ability of the model to describe ozone deposition characteristics, the model was applied to measurements made in chamber experiments of ozone deposition to indoor surfaces. Equation 4-13 was used to determine the chamber ozone concentration at each time step. The measurements are a subset of chamber ozone deposition experiments results from Chapter 3 of this dissertation (Figures 3-5 and 3C-1). Three representative chamber experiments - involving plastic, worn fabric, and carpet were chosen because of their differently shaped ozone profiles. For the fabric, soiled 
wool, the residue characteristics were chosen based on the properties of squalene (molecular weight of $410.7 \mathrm{~g} \mathrm{~mol}^{-1}$, density of $0.86 \mathrm{~g} \mathrm{~cm}^{-3}$, and reaction probability of 4.5 $\times 10^{-4}$ (Wells et al., 2008). For carpet and plastic, the molecular weight and density of oleic acid $\left(\mathrm{MW}_{\text {res }}=292 \mathrm{~g} \mathrm{~mol}^{-1}\right.$ and $\left.\rho_{\text {res }}=1 \mathrm{~g} \mathrm{~cm}^{-3}\right)$ were chosen because these surfaces most likely had some fatty acid residue. However, the reaction probabilities for these two materials were chosen to optimize fit.

Using reasonable parameters as inputs, the model was able to produce an adequate fit to the measured results for plastic and soiled wool with the exception of the early time steps (Figures 4-10a and 4-10b). However, the fit of the modeled to measured data for the used carpet sample was not very good (Figure 4-10c). The most likely reason for this is that there are processes or factors that are not accounted for in the model. One possible process is the diffusion of ozone-reactive compounds through material fibers to their surfaces. The model also may not fully capture the effect of increased surface area on ozone uptake.

The type of data to which this form of the model could be applied also includes other ozone deposition chamber experiments, for example, Morrison and Nazaroff (2000, Figure 1) and Wells et al. (2008, Figure 2). The model may require some additional modifications to be applied to experimental conditions employed in these studies.

\subsection{Implications for indoor environments and conclusions}

In this chapter, a model was developed for predicting ozone consumption by, and gas-phase byproduct formation from, an ozone-reactive residue that partially covers a surface in an indoor environment. The equations developed here build on an established 
model framework of ozone transport and uptake. Model simulations were run that explore the effects of factors such as reactivity of residue, reactivity of base material, air flow conditions, indoor ozone level, and extent of surface coverage on model outputs, which include time-dependent residue consumption, overall (base and residue) reactivity, and byproduct formation. The model is useful for understanding how residues affect ozone consumption and byproduct formation, and what common indoor environmental factors influence those ozone-residue reactions.

In the simulations run with this model, the outcome is that the entire residue is eventually consumed. In a real setting, such as a home, the source of ozone is typically outdoor air, and the indoor ozone concentration tracks the outdoor concentration resulting in a diurnal variation where ozone levels are elevated for a portion of a day, perhaps only a few hours (Nazaroff and Cass, 1986). For residues that are not highly reactive (e.g. $\gamma_{\mathrm{X}}<$ $\left.10^{-4}\right)$, the residue may not be fully consumed within the daily window of ozone intrusion. Furthermore, if the residue is replenished on a daily basis (e.g. by atmospheric deposition of fatty-acid containing particles or sorption of unsaturated semivolatile organic compounds) then the residue could be an ever-present precursor for ozone byproduct formation. 


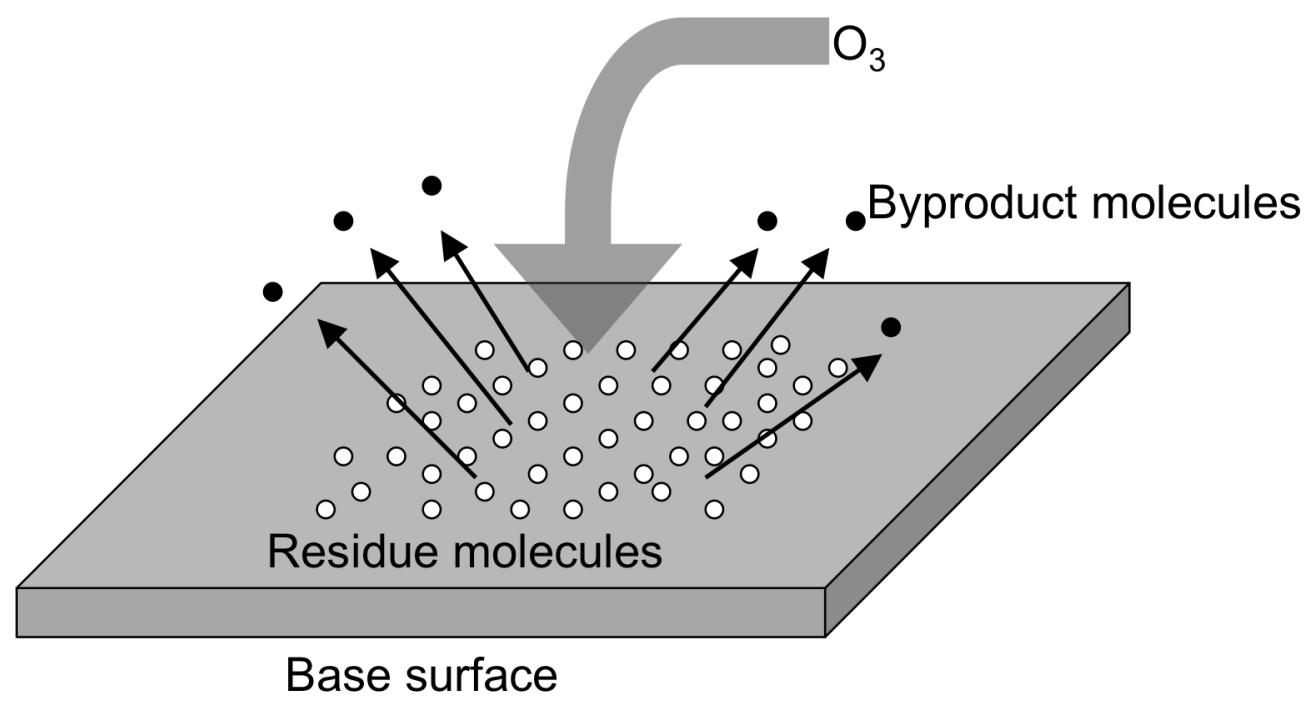

Figure 4-1: Illustration of residue-ozone reaction model. There is a base surface that is nominally smooth and nonporous and that has fixed, intrinsic ozone reactivity. To that surface is added an ozone-reactive residual chemical that partially covers the surface. Ozone is transported to the surface and reacts with the residue and with the base material. Reaction with the residue produces volatile and nonvolatile byproducts. 
(a)

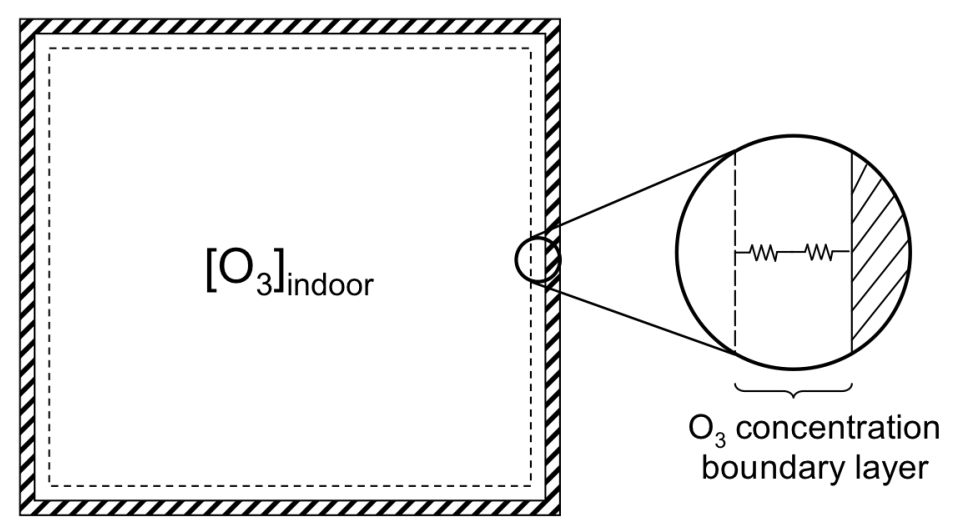

(b)

Two-resistor model

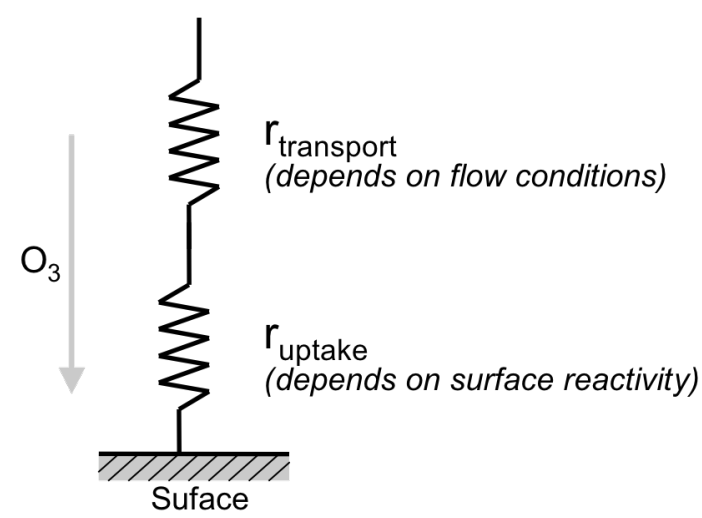

Three-resistor model

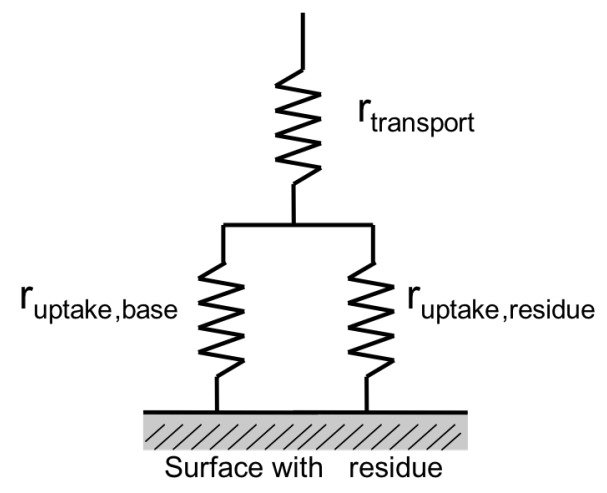

Figure 4-2: Illustration of ozone deposition model. In this model, the core of a room is assumed to be well-mixed so that the indoor ozone concentration $\left(\left[\mathrm{O}_{3}\right]_{\text {indoor }}\right)$ is uniform except for near surfaces where there is a thin concentration boundary layer, as shown in (a). Deposition velocity incorporates the rate of pollutant transport through the boundary layer and the rate of uptake at the surface. Cano-Ruiz et al. (1993) conceptualized these two processes as two resistors in series ( $r_{\text {transport }}$ and $r_{\text {uptake }}$, respectively) for a homogeneous surface, as shown in the first frame of (b). In this chapter, a three-resistor model is proposed to predict ozone deposition velocities with a surface partially covered in a residue, as shown in the second frame of (b). The two resistors in parallel near the surface represent the competing uptake of ozone by the residue ( $\left.r_{\text {uptake, residue }}\right)$ and the base material ( $\left.r_{\text {uptake,base }}\right)$ of the surface. 


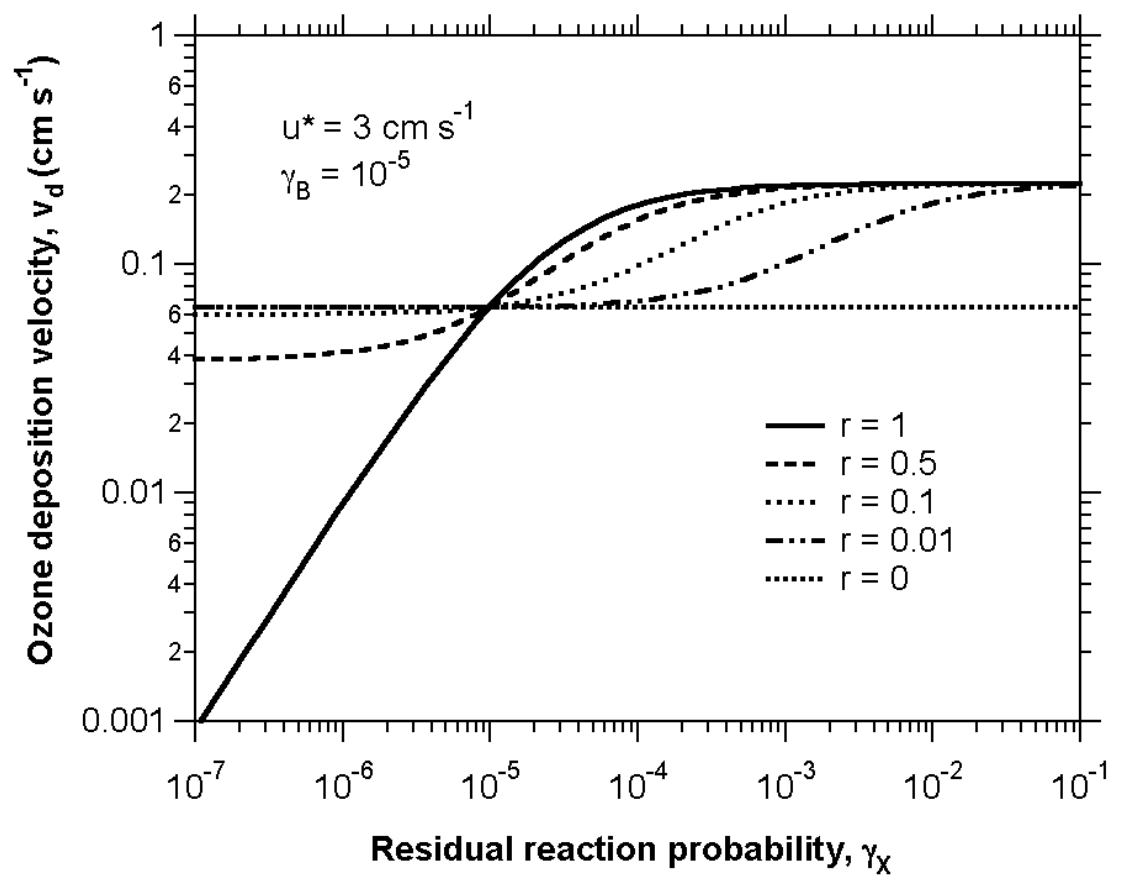

Figure 4-3: The effect of residual reactivity, $\gamma_{X}$, and residual coverage, $r$, on overall ozone deposition, $v_{d}$, to the surface. Equations 4-5 and 4-6 were used to create the plot. 
Flat surface

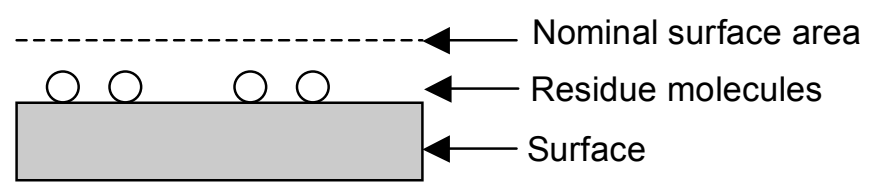

Textured surface

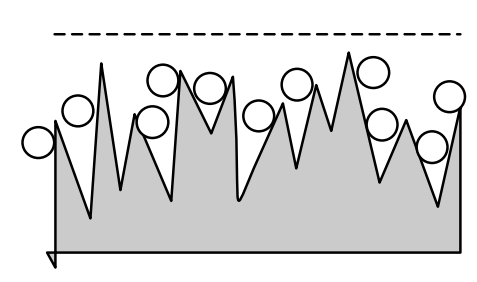

Figure 4-4: Illustration of flat and textured surfaces. Actual surface area is greater than nominal surface area for textured surfaces. 

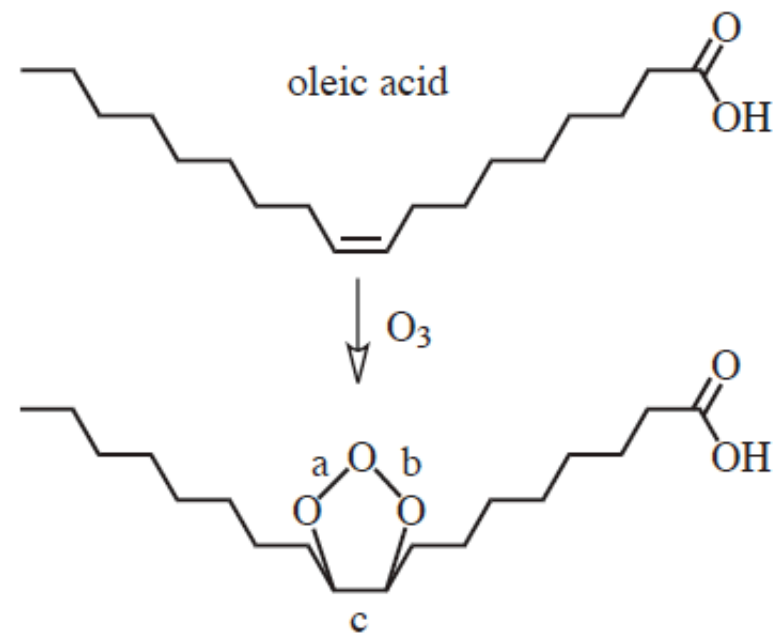

primary ozonide,

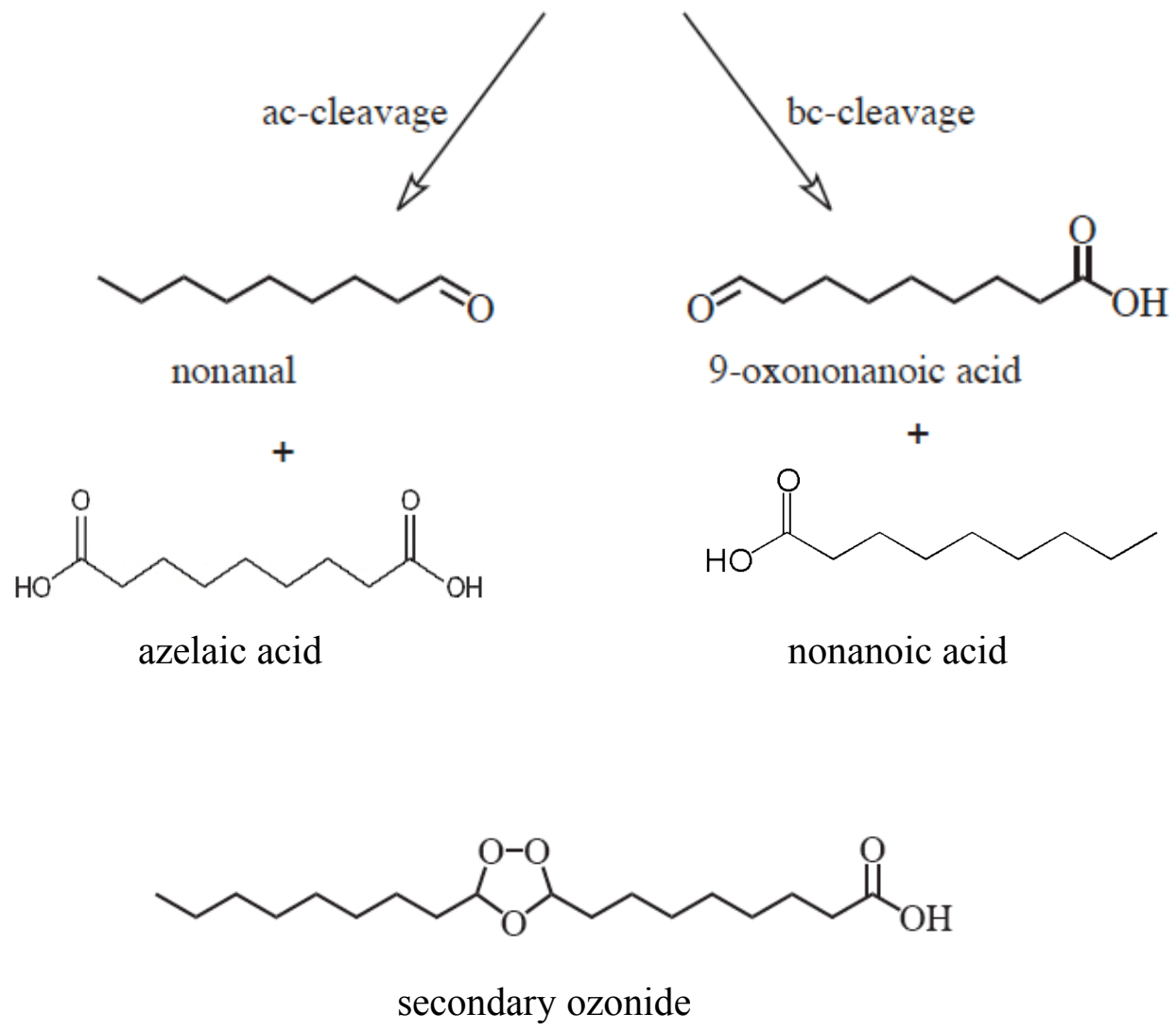

Figure 4-5: Oleic acid and its oxidation products (adapted from Zahardis and Petrucci, 2007). The formation of a secondary ozonide is possible in solution (liquid oleic acid) when fragments the ozone-oleic acid reaction stay in close proximity to one another. 


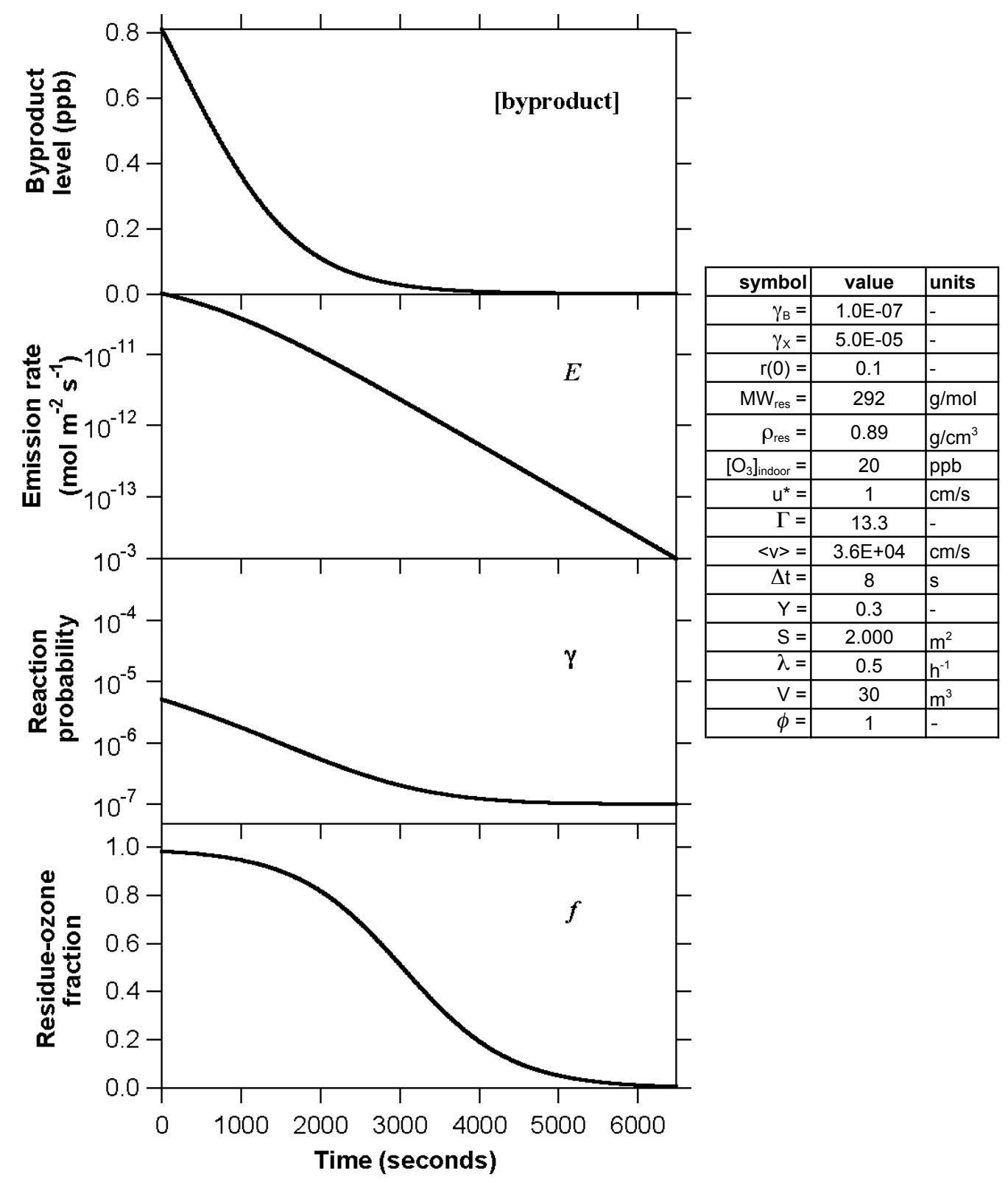

Figure 4-6a: Parameter inputs and model results for oleic acid example. Note that the axes for $f$ and [byproduct] are on a linear scale and the axes for $E$ and $\gamma$ are on a logarithmic scale. 


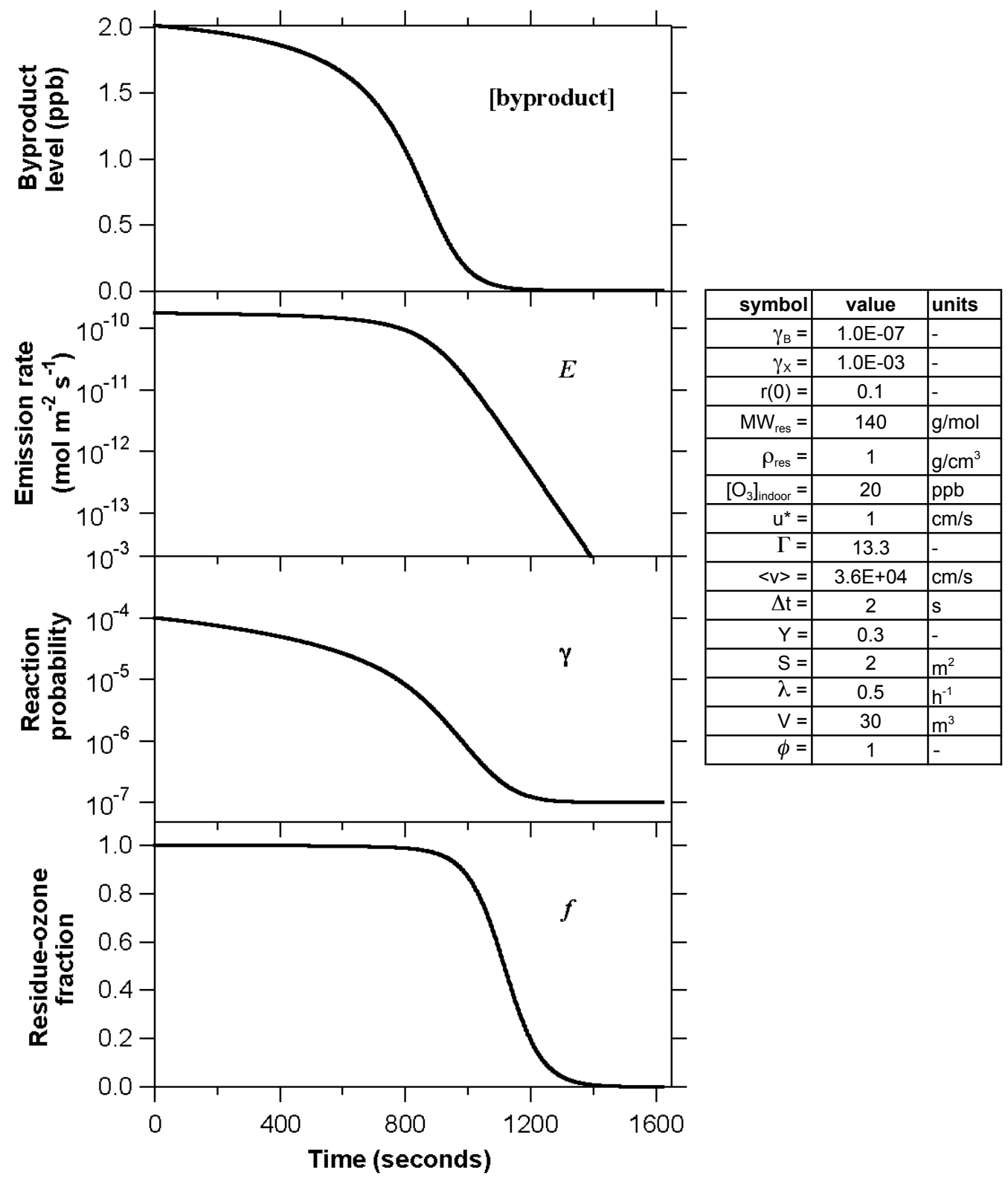

Figure 4-6b: Parameter inputs and model results for hypothetical cleaning product example. Note that the axes for $f$ and [byproduct] are on a linear scale and the axes for $E$ and $\gamma$ are on a logarithmic scale. 
(a)

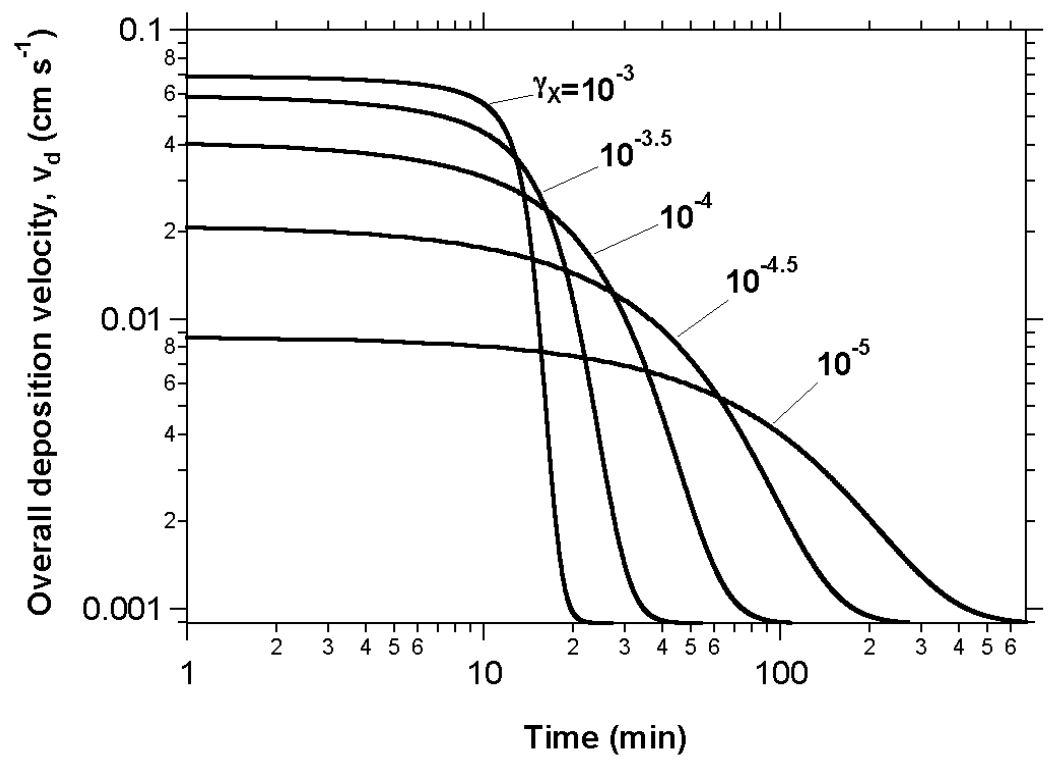

(b)

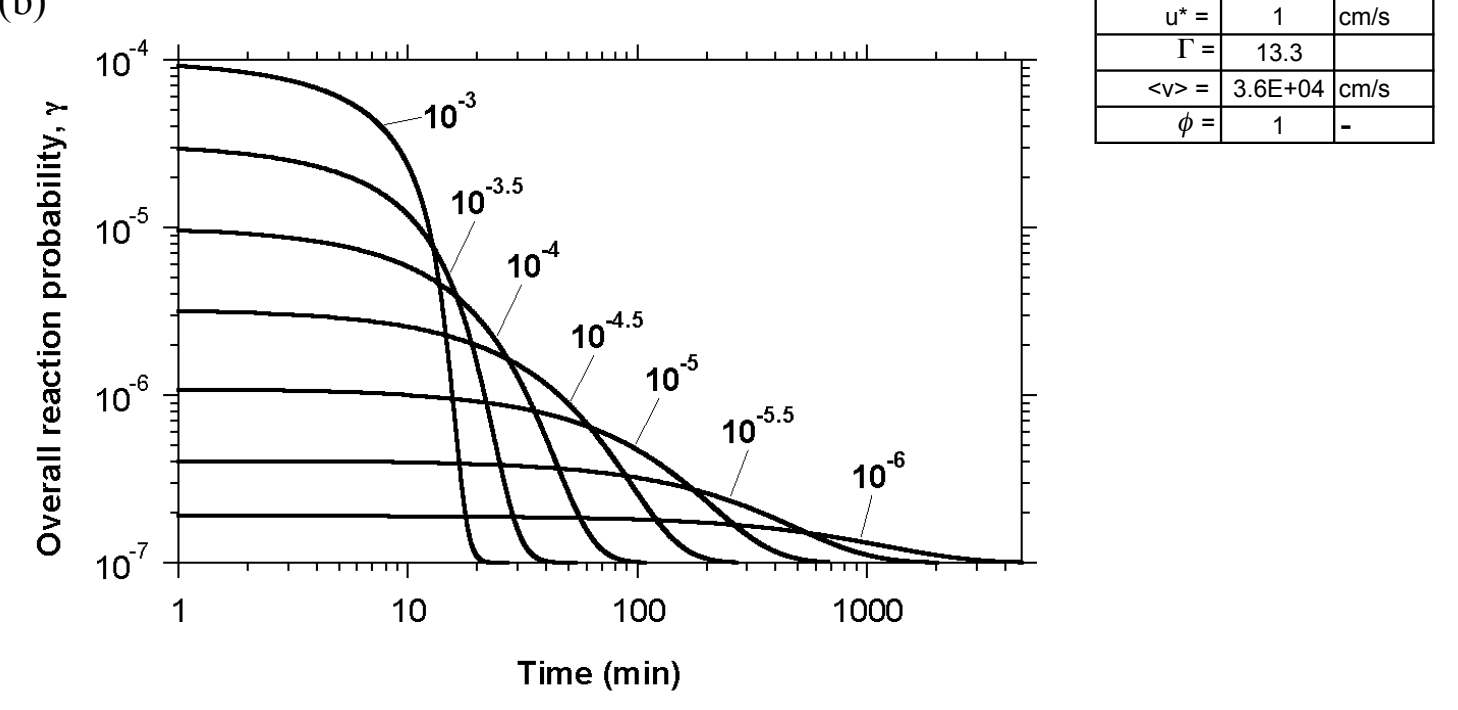

Figure 4-7: Time-dependent (a) overall deposition velocity $\left(v_{d}\right)$ and (b) overall reaction probability $(\gamma)$ to a surface with base reactivity, $\gamma_{B}=10^{-7}$, and varying residue reaction probability $\left(\gamma_{X}\right)$. Residual reaction probabilities are indicated on the plot. Other model parameters are shown in the table to the right. 


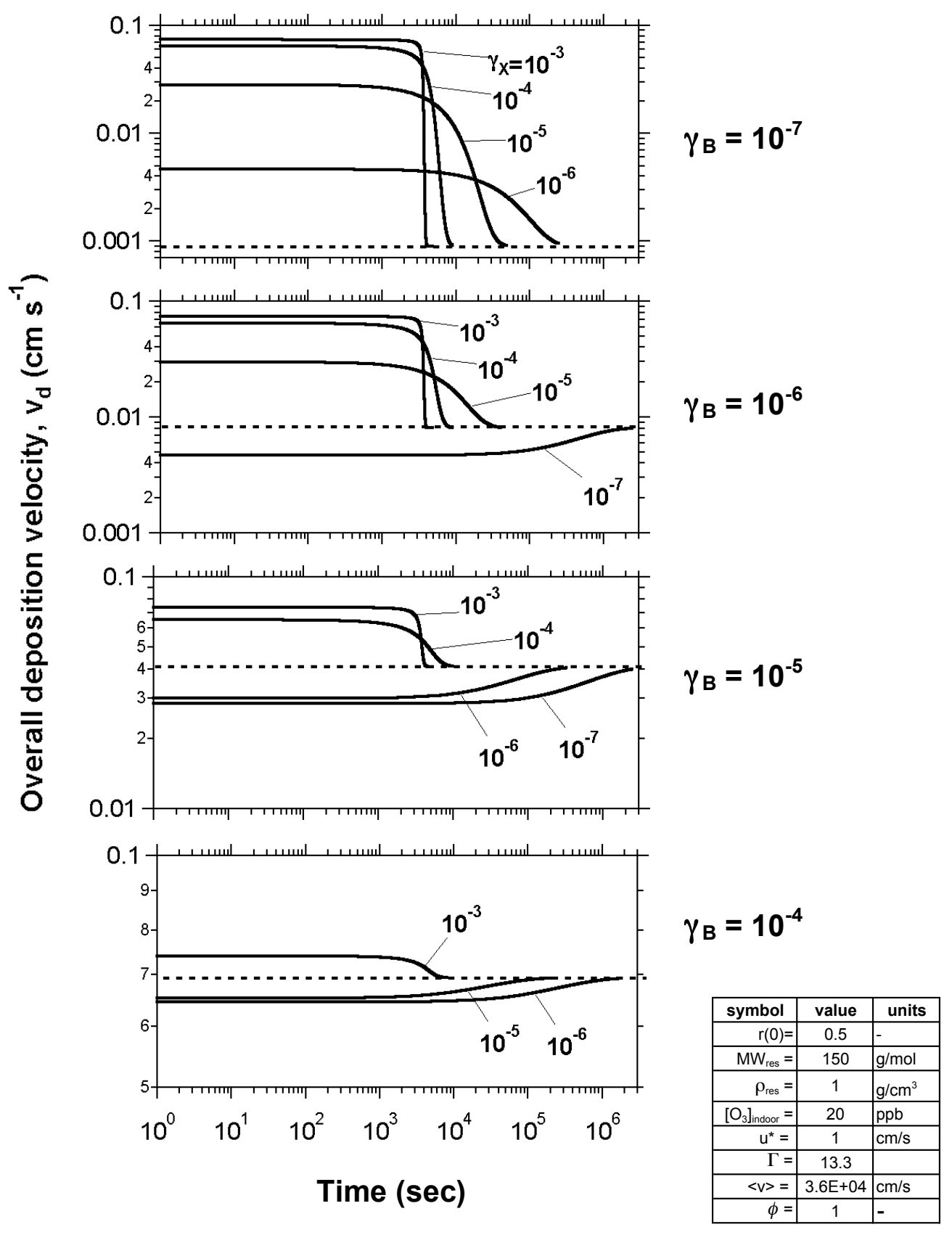

Figure 4-8: Time-dependent deposition velocity $\left(v_{d}\right)$ with various base reactivities $\left(\gamma_{B}\right)$ and varying residue reaction probability $\left(\gamma_{X}\right)$ Residual reaction probabilities are indicated on the plot. Other model parameters are shown in the table to the right. Note that the yaxis range varies. The dashed line indicates the "inherent" deposition velocity of the base material (or $\left.\gamma_{X}=\gamma_{B}\right)$. 


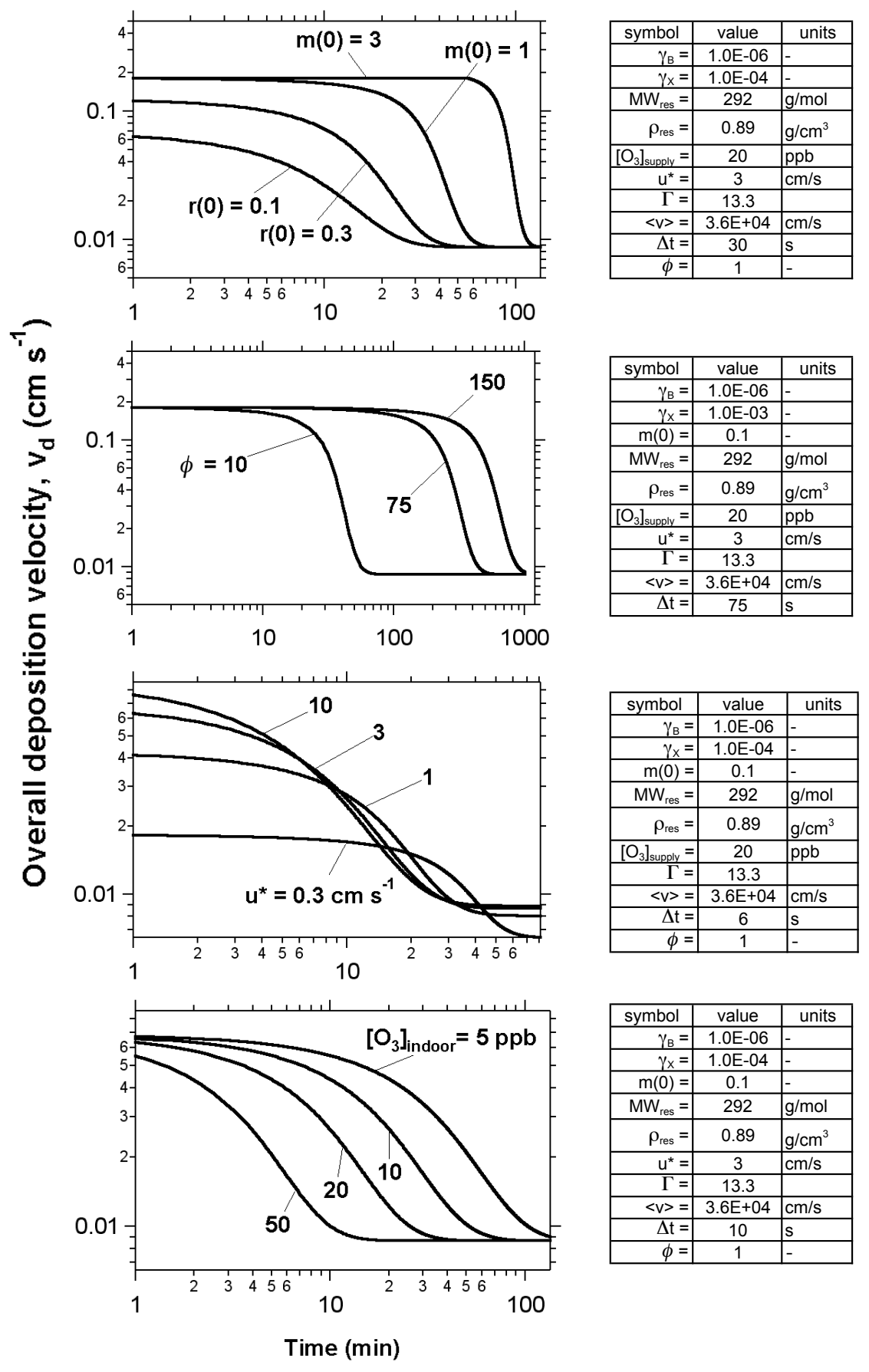

Figure 4-9: Effects on deposition velocity $\left(v_{d}\right)$ of various model parameters: initial monolayer coverage $(m(0))$ or initial fractional surface coverage $(r(0))$, surface multiplication factor $(\phi)$, friction velocity $\left(u^{*}\right)$, and steady indoor ozone level $\left(\left[\mathrm{O}_{3}\right]_{\text {indoor }}\right)$ Other model parameters are listed in the tables to the right. 


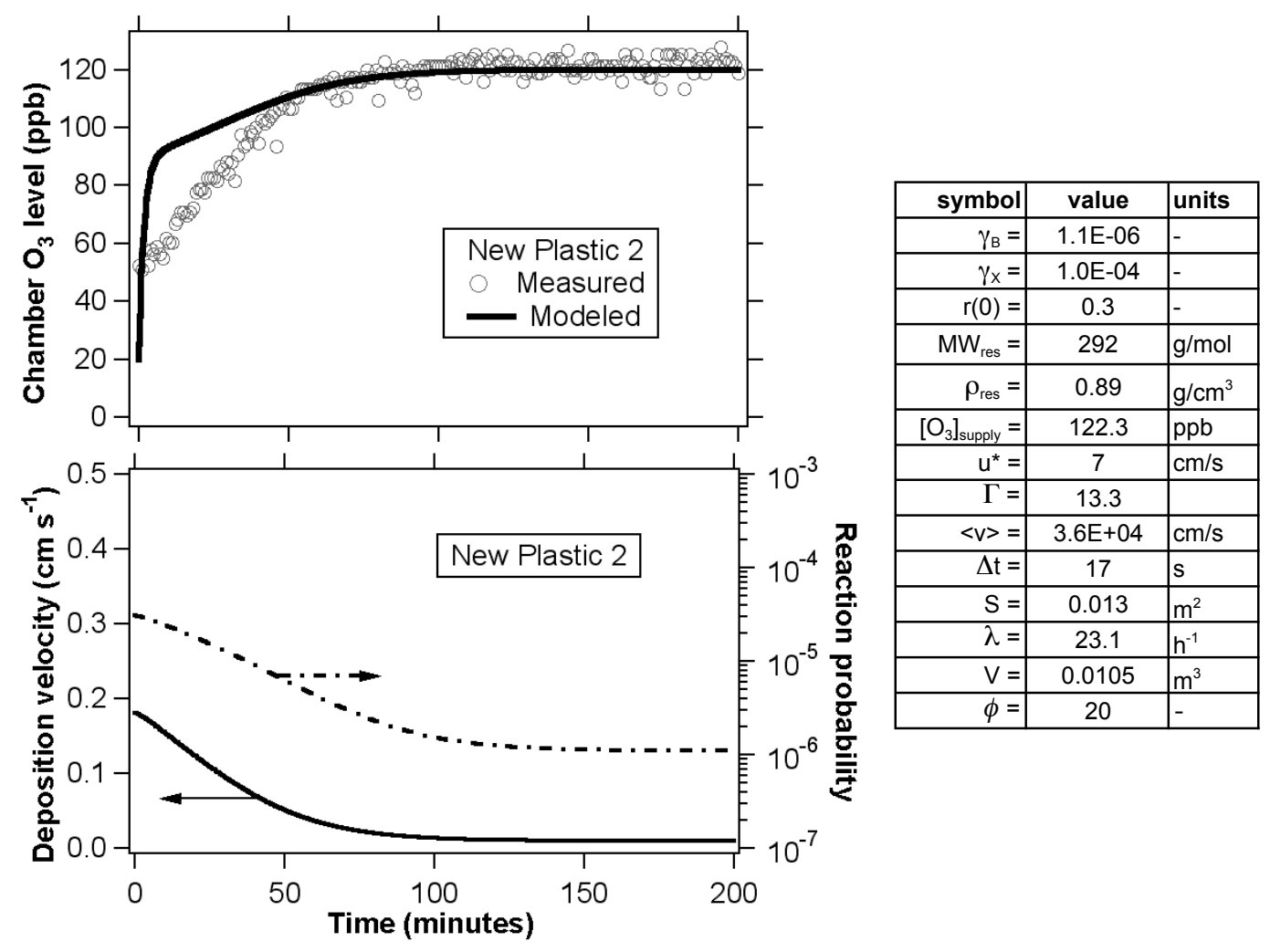

Figure 4-10a: Model fit to ozone data measured in a chamber experiment with new plastic. Ozone level, deposition velocity, reaction probability were modeled using the parameters shown in the table to the right. 


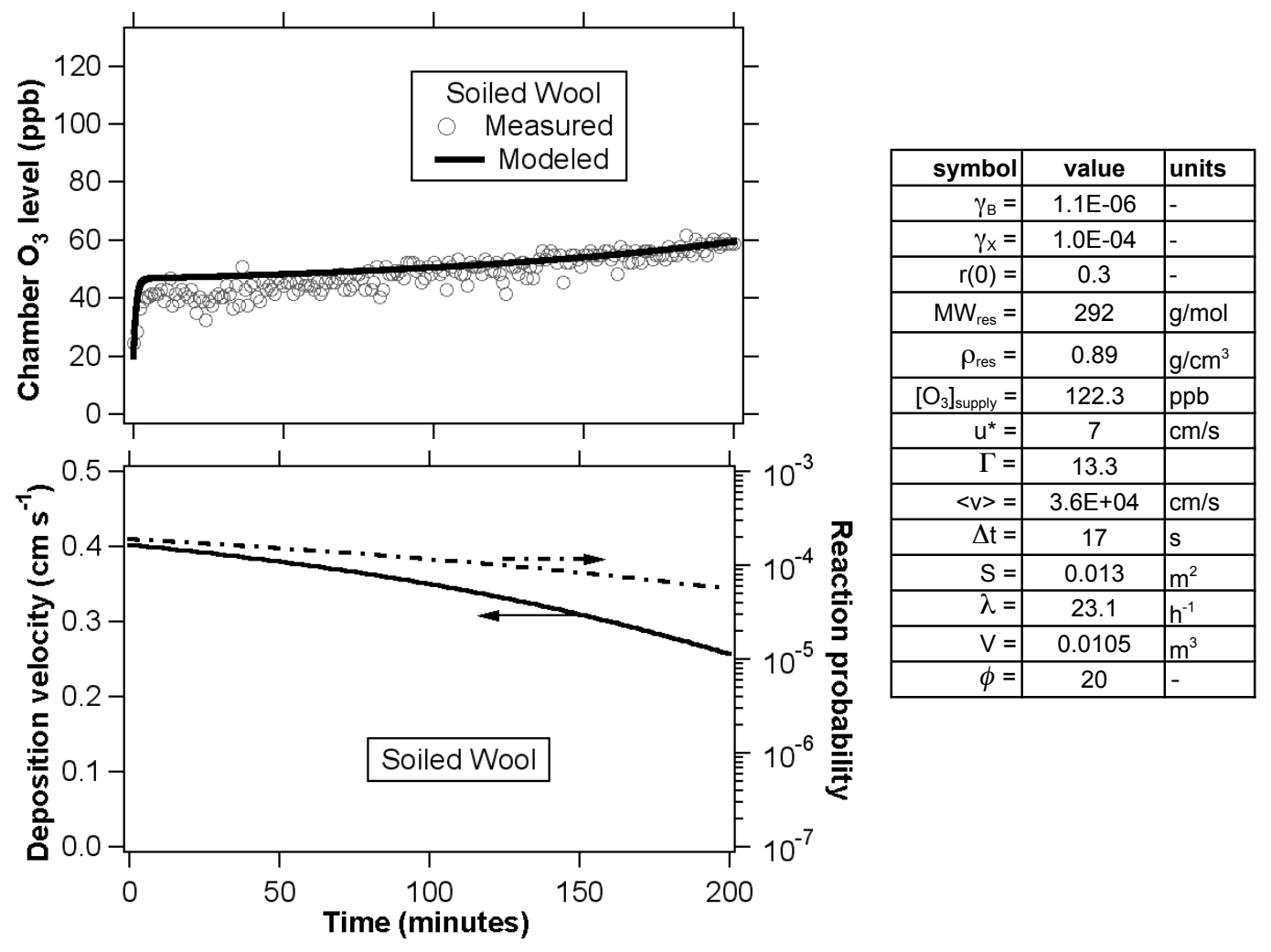

Figure 4-10b: Model fit to ozone data measured in a chamber experiment with wool that had been worn near the skin for several hours before exposure to ozone. Ozone level, deposition velocity, reaction probability were modeled using the parameters shown in the table to the right. 


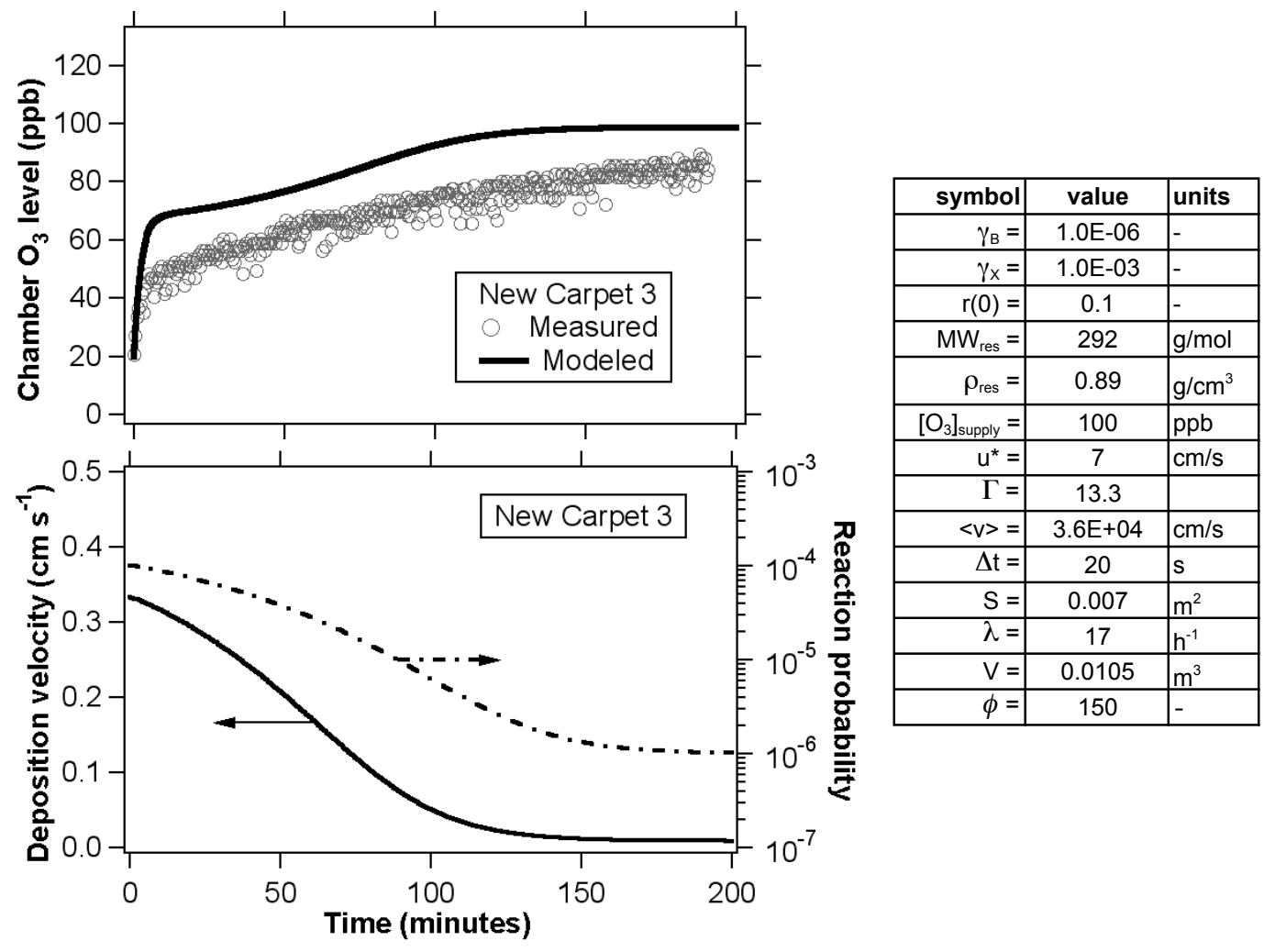

Figure 4-10c: Model fit to ozone data measured in a chamber experiment with new carpet. Ozone level, deposition velocity, reaction probability were modeled using the parameters shown in the table to the right. 


\section{Investigation of ozone-induced decomposition of surface- bound permethrin}

\subsection{Introduction}

"Disinsection" is the practice of spraying passenger aircraft interiors with insecticide to prevent the spread of disease vectors and invasive species. This practice is controversial because of the risk associated with exposing passengers and crew to insecticides. Regular insecticide spraying was discontinued in the United States in 1979, but several countries, at least 21, still permit or require disinsection (NRC, 2002; DOT, 2007). In the countries where disinsection is practiced, their principal concern is avoiding the spread of malaria (NRC, 2002). Although no pesticide approved for use in an occupied aircraft cabin, several hundred pesticides are registered for use on aircraft in the United States (Maddalena and McKone, 2008). Planes that have been disinsected travel worldwide (i.e., even to countries where disinsection is not allowed). Consequently, exposure to insecticides and their degradation products is a potential concern for all cabin passengers and crew.

Publicly available data on the type and amount of insecticides used in aircraft cabins are sparse, but they suggest that the most commonly used aircraft insecticides, domestically and internationally, are permethrin and d-phenothrin (Maddalena and McKone, 2008). These are two four of the pesticides that recommended for this purpose by the World Health Organization (WHO): d-phenothrin, permethrin, resmethrin, and bioresmethrin (WHO, 1995). All of these pesticides belong to the chemical class called 
pyrethroids; pyrethroids are a synthetic forms of pyrethrins, which are the natural insecticides produced by the chrysanthemum flower (NPTN, 1998).

Permethrin is used as a "residual" pesticide (i.e., designed for long-lasting effectiveness) and d-phenothrin as a "non-residual" pesticide (i.e., only for immediate effectiveness). Chemically, the difference between the two compounds is the substitution of two methyl groups $\left(-\mathrm{CH}_{3}\right)$ on the phenothrin molecule with two chlorine atoms on the permethrin molecule (see Figure 5-1a). Chlorine substitution makes permethrin more likely to partition to surfaces and more resistant to degradation. These characteristics lead to improved persistence, a feature that is useful for residual disinsection applications.

Direct risks of adverse health effects are posed by pesticide exposure via inhalation, ingestion, and dermal uptake. Another potential hazard associated with pesticide use is exposure to the degradation products of the primary chemicals, and one potential route of degradation is oxidation. One example of such a concern arises with malathion, which is oxidized to the more toxic compound, malaoxon, by reaction with the hydroxyl radical (Brown et al., 1993). In the aircraft cabin environment, ozone is of concern as an oxidizing agent. When ozone reacts with an alkene, a primary carbonyl and a biradical are formed (Atkinson and Carter, 1984). (The hydroxyl radical is also formed; the significance of this point is discussed more in Section 5.3.3.) For an asymmetrical alkene, there are two possible primary carbonyls that could be formed, and the branching ratio of the primary carbonyls is dependent on the neighboring functional groups (Grosjean and Grosjean, 1997). Permethrin possesses a terminal dichlorovinyl group, a carbon-carbon double bond with two chlorines atoms (see Figure 5-1a), and cleavage of this double bond by ozone would be anticipated to produce two primary carbonyls - a 
carboxaldehyde and phosgene (Dowideit and von Sonntag, 1998; Ruzo et al., 1986; Class, 1991). Formation of the latter is illustrated in Figure 5-1b. Phosgene is a highly toxic gas, infamous for its use as a chemical warfare agent in World War I.

Ozone, a strong oxidant, is commonly present in aircraft cabins. The source of ozone on or near the ground is polluted ambient air. Of greater concern is that, during flight, planes commonly encounter naturally elevated ozone levels originating in the stratosphere. Regulations are in place to prevent excessive ozone levels in the passenger cabins of commercial aircraft. However, ozone levels on the order of 10-100 ppb are commonly encountered either on domestic flights when planes are not equipped with ozone converters or on transoceanic flights (Spengler et al., 2004; Bhangar et al., 2008). Several recent cabin air quality studies have established that a significant fraction of the ozone that enters the cabin, approximately $60-80 \%$, is consumed by reactions with cabin interior surfaces. Studies have measured products of ozone-initiated chemistry (Wisthaler et al, 2005; Tamás et al., 2006; Weschler et al., 2007; Chapter 3 of this dissertation). The chemical analysis methods in those studies were not capable of detecting phosgene, and no known attempts have been made to measure phosgene during flight or under simulated cabin conditions.

Formation of phosgene from permethrin oxidation has been predicted (Ruzo et al., 1986; Class, 1991), but not measured. To date, the branching ratio or yield of phosgene formed from ozonation of a dichlorovinyl group on a surface has been measured in only one study, which was conducted with cypermethrin (Segal-Rosenheimer and Dubowski, 2007). Cypermethrin is another surface-bound insecticide that contains the dichlorovinyl group. The researchers used a "thick" layer (much greater than one monolayer) of 
insecticide and very high concentrations of ozone ( $3 \mathrm{ppm}$ to $50 \mathrm{ppm}$ ) in an apparatus with coupled long path and attenuated total reflectance Fourier transform infrared spectroscopy (LP-ATR-FTIR). The yield, defined as moles of phosgene formed per mole of permethrin reacted, was estimated to be $6-10 \%$ in those experiments. Reaction of ozone with a dichlorovinyl-containing pesticide has not been measured under typical cabin conditions.

\subsection{Objective and approach}

The objective of this study was to determine if phosgene could be formed at levels of concern in the aircraft cabin environment from the reaction of ozone and permethrin. First, the potential for phosgene formation in cabin-relevant conditions was investigated by conducting a literature search and using modeling estimates. Next, laboratory experiments were performed in which permethrin-sprayed cabin materials were exposed to ozone at cabin relevant conditions. As part of the experiments, a derivatization method was developed to detect low levels of phosgene. Then, results from the experiments were incorporated into a model of ozone transport and uptake to estimate the upper limit of phosgene levels possible in the cabin.

\subsection{Characterization of potential for phosgene formation}

In this section, the available information about phosgene, permethrin, and permethrin-ozone reactions is reviewed. Cabin ozone levels and permethrin surface concentrations on cabin surfaces are determined from the literature, and this information is used to estimate whether the levels of these precursors are sufficient for phosgene formation at levels of concern. The cabin is modeled to determine potential levels of 
phosgene assuming that the ozone and permethrin readily react. The established health standards for phosgene are identified, and the pertinent chemical attributes of permethrin and phosgene are explored.

\subsubsection{Pyrethroid surface concentrations}

World Health Organization (1995) guidelines for disinsection indicate that permethrin can be applied as an aerosol from a spray can or as an aqueous solution from a low-pressure sprayer. The two aerosol treatment procedures, "pre-flight" and "preembarkation", are designed to deliver $0.35 \mathrm{~g}$ of solution per $\mathrm{m}^{3}$ using aerosol cans containing $2 \%$ permethrin (in the case of "pre-embarkation," the spray also contains $2 \%$ phenothrin). These procedures are performed when the cabin is unoccupied. The "preflight" treatment is to be coupled with "blocks away", "top of descent", or "on arrival" treatment in which the occupied cabin is sprayed with a $2 \%$ phenothrin aerosol. Based on studies where cabin insecticide residue levels have been measured, most insecticide is concentrated on upward facing surfaces, which is likely because of gravitational settling of spray droplets. Using the estimated typical horizontal surface area per volume in the cabin $\left(\sim 1 \mathrm{~m}^{2} \mathrm{~m}^{-3}\right)$ and assuming that all of the permethrin sprayed lands on these surfaces, the expected surface concentration of permethrin would be $\sim 700 \mathrm{ng} \mathrm{cm}^{-2}$.

Airlines that frequently fly to regions requiring disinsection may opt to perform "residual" treatment, where the cabin is sprayed with a much higher concentration of permethrin, but less frequently. The guidelines state that an air spray gun or a pressure garden sprayer may be used to deliver an aqueous solution of $2 \%$ permethrin in water to all surfaces. This procedure is performed in an unoccupied cabin approximately every 
eight weeks. This treatment is designed to deliver $0.2 \mathrm{~g}$ of permethrin per $\mathrm{m}^{2}(20,000 \mathrm{ng}$ $\mathrm{cm}^{-2}$ ). In Figure 5-2, targeted surface concentrations are compared to surface concentrations measured in several cabin studies. Pertinent details of the studies are discussed in the following paragraphs. For reference, the estimated monolayer of permethrin molecules on a smooth surface is $\sim 100 \mathrm{ng} \mathrm{cm}^{-2}$. The surface concentration corresponding to monolayer coverage was determined by assuming that the permethrin molecules occupied a cubic volume equal to the molecular weight divided by the liquid density. With a molecular weight of $391 \mathrm{~g} \mathrm{~mol}^{-1}$ and a density of $1.2 \mathrm{~g} \mathrm{~cm}^{-3}$, the volume per molecule is $0.54 \mathrm{~nm}^{3}$, which corresponds to a cube length of $0.81 \mathrm{~nm}$. Using a footprint of $0.66 \mathrm{~nm}^{2}$ per molecule, a surface concentration of $98 \mathrm{ng} \mathrm{cm}^{-2}$ would be required for monolayer coverage.

Berger-Preiss et al. (2004 and 2006) measured insecticide concentrations in the gas-phase and on surfaces during and after spraying pyrethroid insecticides in planes parked on the tarmac. The 2004 study aimed to replicate in-flight spraying (e.g., "top-ofdescent"), and reported a median value of $\sim 50 \mathrm{ng} \mathrm{cm}^{-2}$ of pyrethroids (permethrin or pyrethrin) on upward surfaces. The 2006 study simulated a "pre-embarkation" treatment procedure, i.e. spraying shortly before passengers board, and reported typical values of $\sim 100-1200 \mathrm{ng} \mathrm{cm}^{-2}$ of d-phenothrin on "mainly horizontal areas."

A recent investigation by NIOSH of pesticide illness among flight attendants reports nonincident levels of permethrin associated with residual disinsection measured in Boeing 747-400 aircraft cabins by staff at an unnamed airline (Sutton et al., 2007). The dataset contains measurements from 64 surface wipe samples and 23 samples of fabrics and other cabin materials. Permethrin levels on these surfaces and materials varied by six 
orders of magnitude, with a median of $160 \mathrm{ng} \mathrm{cm}^{-2}$, a mean of $59,000 \mathrm{ng} \mathrm{cm}^{-2}$, and a maximum of 3,600,000 $\mathrm{ng} \mathrm{cm}{ }^{-2}$. "Permethrin was detected on aircraft cabin surfaces up to 28 hours after the pesticide was applied. No samples were collected later than 28 hours after the pesticide application."

An informal investigation by a flight attendant using a wipe method found a surface concentration of 170 to $690 \mathrm{ng} \mathrm{cm}^{-2}$ of permethrin on a B747-400 that had been subject to residual spray treatment (NRC, 2002).

Limited data suggest that residual spraying of insecticides would commonly produce residual levels on upward surfaces in aircraft cabins of the order of 100-1000 ng $\mathrm{cm}^{-2}$ for permethrin, although much higher levels may also occur. Airborne levels of permethrin are expected to be low owing to its low vapor pressure of $2.8 \times 10^{-11} \mathrm{~atm}$ (EPA, 2006). In a study measuring airborne concentrations of semivolatile organic compounds on four commercial flights, Spicer et al. (2004) found measurable airborne concentrations of cis-permethrin on one flight $\left(0.9 \mathrm{ng} \mathrm{m}^{-3}\right)$ and of trans-permethrin on two flights (1.1 and $\left.2.0 \mathrm{ng} \mathrm{m}^{-3}\right)$. Using an airborne concentration of $1 \mathrm{ng} \mathrm{m}^{-3}$, a surface loading of $100 \mathrm{ng} \mathrm{cm}^{-2}$, and typical dimensions of a plane, the expected mass of permethrin on surfaces would be approximately six orders of magnitude higher than in the air.

\subsubsection{Potential phosgene formation}

To estimate the phosgene level that may be produced in the cabin environment, one can apply a steady-state material balance model. For estimation purposes, phosgene is assumed to be generated by means of one dominant process: ozone reaction with 
upward facing surfaces. The concentration of permethrin on these surfaces is set at 100 $\mathrm{ng} \mathrm{cm}^{-2}$; hence, one can assume for rough estimation purposes that the horizontal surfaces are completely covered in at least one monolayer of permethrin. This is a crude description because there is a much greater surface area associated with cabin materials than the nominal surface area. The rate of phosgene generation, $R_{p h o s}$, is expressed in moles per time and can be approximated as the product of several terms:

$$
R_{\text {phos }}=C_{\text {ozone }}\left(v_{d} \times S_{h} / V\right) Y
$$

In this expression, $C_{\text {ozone }}$ represents the ozone level in the cabin (ppb), $v_{d}$ is the deposition velocity for ozone reaction on cabin surfaces $\left(\mathrm{m} \mathrm{h}^{-1}\right), S_{h}$ is the surface area of upward-facing horizontal surfaces in the cabin $\left(\mathrm{m}^{2}\right), V$ is the cabin volume $\left(\mathrm{m}^{3}\right)$, and $Y$ represents the yield of phosgene formation from ozone reaction with permethrin. The quantity $\left(v_{d} \times S / V\right)$ is a first order loss rate coefficient for ozone, and adding yield to that term $\left(v_{d} \times S / V \times Y\right)$ makes it a first order rate coefficient for byproduct formation.

The rate of removal of phosgene by means of ventilation, $L_{p h o s}$, in units of moles per time, may be represented by equation 5-2, which reflects a steady-state material balance on phosgene.

$$
L_{p h o s}=C_{p h o s} \lambda
$$

Here $C_{p h o s}$ represents the phosgene level in the cabin air (ppb) and $\lambda$ is the cabin air exchange rate $\left(\mathrm{h}^{-1}\right)$. Setting $R_{\text {phos }} \sim L_{\text {phos }}$, substituting in the expressions from equations 5-1 and 5-2, and rearranging, leads to this summary expression for estimating the phosgene level:

$$
C_{\text {phos }} \approx \frac{C_{\text {ozone }} v_{d} \frac{S_{h}}{V} Y}{\lambda}
$$


One can now substitute some representative values for the various parameters in equation 5-3. A typical peak-hour ozone level in the cabin environment is $30 \mathrm{ppb}$ (Bhangar et al., 2008). Based on chamber and simulated cabin experiments, a typical value of $v_{d}$ is $\sim 3.0 \mathrm{~m} \mathrm{~h}^{-1}$ (Tamás et al., 2006). A reasonable estimate for the horizontal surface-to-volume ratio for the cabin environment would be, $S_{h} / V \sim 1 \mathrm{~m}^{2} \mathrm{~m}^{-3}$. A representative value for fresh cabin air-exchange rate $(\lambda)$ is $\sim 10 \mathrm{~h}^{-1}$ (NRC, 2002). SegalRosenheimer and Dubowski (2007) estimated that the oxidative yield $(Y)$ of phosgene from cypermethrin was $\sim 5-10 \%$. I use a value of 0.1 for this preliminary estimate. Thus, I estimate that a phosgene level of $1 \mathrm{ppb}$ might be sustained in the aircraft cabin environment as a result of ozone-induced oxidation of permethrin as shown in the following calculation.

$$
C_{\text {phos }} \sim \frac{30 \mathrm{ppb} \times 3 \mathrm{~m} \mathrm{~h}^{-1} \times 1 \mathrm{~m}^{2} \mathrm{~m}^{-3} \times 0.1}{10 \mathrm{~h}^{-1}} \sim 1 \mathrm{ppb}
$$

Higher levels would certainly be possible under unfavorable conditions, in which, for example, the ozone level was above $30 \mathrm{ppb}$ or the yield was higher than 0.1 . Note that this analysis assumes that permethrin reacts with ozone at a rate that is controlled by external mass transfer of ozone to the cabin surfaces, as expressed through the deposition velocity. This is approximately the maximum rate at which the reaction could occur; slow reaction kinetics between ozone and permethrin would result in lower cabin phosgene levels.

\subsubsection{Reactivity of permethrin}

Permethrin's carbon-carbon double bond makes it vulnerable to attack by ozone. A program called "AOPwin" calculates gas-phase reaction rate constants of organic 
compounds with ozone and with the hydroxyl radical, based on chemical structure. The Atmospheric Oxidation Program (AOP) was developed by Meylan and Howard (1993). It is part of the EPA's Estimation Program Interface (EPI) Suite, and the program can be downloaded from the EPA's exposure assessment models and tools website (www.epa.gov/oppt/exposure/pubs/episuite.htm). While AOPwin was not developed for very low vapor pressure compounds that tend to partition to surfaces, the AOPwin evaluation of the ozone-permethrin reaction rate can still be used to crudely estimate the reactivity of surface-bound permethrin with ozone. The calculated reaction rate for ozone and permethrin is $2.3 \times 10^{-19} \mathrm{~cm}^{3}$ molec $^{-1} \mathrm{~s}^{-1}$. For reference, the calculated reaction rate for ozone and phenothrin, which has methyl groups rather than chlorine atoms adjacent to the double-carbon bond, is $4.3 \times 10^{-16} \mathrm{~cm}^{3} \mathrm{molec}^{-1} \mathrm{~s}^{-1}$, roughly three orders of magnitude higher. Owing to the presence of strong electron-withdrawing chlorine atoms, the double bond in permethrin is much less electron-rich than the double bond in phenothrin, which greatly reduces its reactivity with electrophiles such as ozone. To illustrate the effect of chlorine substitution near the unsaturated bond, Table 5-1 shows ozone reaction rates of progressively more chlorinated ethane-based molecules. The substitution of one chlorine reduces the reactivity of ethene by $35 \times$ and substituting two chlorine atoms for hydrogen atoms reduces the reactivity of ethene by $240 \times$. However, the presence of a surface may hinder or catalyze reactions; it is unknown whether being partitioned to a surface will increase or inhibit permethrin's reactivity relative to its estimated gas-phase reaction rate.

Based on AOPwin, the reaction of ozone with permethrin is expected to be slow owing to the electron-withdrawing effect of the chlorine atoms near the double bond. However, the reaction of ozone with an alkene is a chain-initiating reaction that produces 
other oxidants such as the hydroxyl radical $(\mathrm{OH})$. $\mathrm{OH}$ reacts much more rapidly than ozone with alkenes, and this is reflected in the gas-phase reaction rate predicted by AOPwin of $2.3 \times 10^{-11} \mathrm{~cm}^{3} \mathrm{molec}^{-1} \mathrm{~s}^{-1}$ for permethrin with $\mathrm{OH}$, i.e., eight orders of magnitude faster than predicted for permethrin with ozone. It is likely that $\mathrm{OH}$ would be formed in the reaction of ozone with other unsaturated compounds on indoor surfaces, and it is feasible that $\mathrm{OH}$ could then react with permethrin. One study measured $\mathrm{OH}$ indoors in the presence of ozone and limonene, and the $\mathrm{OH}$ level was $\sim 10^{-5} \mathrm{ppb}$ with $\sim 100 \mathrm{ppb} \mathrm{O}_{3}$ (Weschler and Shields, 1997). Hence, in that case there was approximately seven orders of magnitude difference between the ozone and $\mathrm{OH}$ levels. $\mathrm{OH}$ could be important for permethrin oxidation if the proportional difference between the concentrations of $\mathrm{O}_{3}$ and $\mathrm{OH}$ is less than the proportional difference between reaction rates of ozone-permethrin and $\mathrm{OH}-$ permethrin. Oxidation of alkenes by $\mathrm{OH}$ and $\mathrm{O}_{3}$ can generate similar products via similar mechanisms (Atkinson and Arey, 2003). For example, Feigenbrugel et al. (2006) detected phosgene formation from the gas-phase reaction of $\mathrm{OH}$ with dichlorvos, an organophosphorus pesticide that has a dichlorovinyl group. Thus, phosgene might be expected to be a product of the OH-permethrin reaction.

\subsubsection{Background information for phosgene}

Phosgene is infamous for its use as a chemical warfare gas in World War I (CDC, 2006). The dominant exposure route for phosgene is inhalation. It is a pulmonary agent that causes respiratory and cardiovascular failure at high concentrations. At lower concentrations it can cause eye and throat irritation, difficulty breathing, and coughing. According to the Agency for Toxic Substances and Disease Registry, phosgene is not 
water soluble, and it hydrolyzes slowly, which means that phosgene can be inhaled more deeply into the lung and have a delayed effect. Milder symptoms may be immediate and more severe symptoms may be delayed for up to two days (ATSDR, 2007).

Phosgene is still used today as an industrial feedstock chemical for the manufacture of plastics and pesticides (CDC, 2006). There are public-health concerns about environmental exposure associated both with routine releases and with industrial or transportation accidents, and thus several exposure limits have been established.

\subsubsection{Phosgene exposure guidelines}

The US Environmental Protection Agency's Integrated Risk Information System lists a reference concentration (RfC) for chronic inhalation exposure to phosgene of 0.3 $\mu \mathrm{g} \mathrm{m}^{-3}$, which corresponds to $\sim 0.08 \mathrm{ppb}$ under standard exposure conditions (sea-level pressure, room temperature). "The RfC is an estimate (with uncertainty spanning perhaps an order of magnitude) of a continuous inhalation exposure to the human population (including sensitive subgroups) that is likely to be without an appreciable risk of deleterious effects during a lifetime" (EPA, 2005).

The California Office of Environmental Health Hazard Assessment has established an acute reference exposure level of $4 \mu \mathrm{g} \mathrm{m}^{-3}$ or $\sim 1 \mathrm{ppb}$ for phosgene. This level applies to a one-hour exposure period. Exposure at this level or below is considered to be adequate to protect against mild adverse effects from phosgene exposure (OEHHA, 1999).

Occupational exposure limits are typically set at much higher values than are environmental exposure limits. The US Occupational Safety and Health Administration's 
Permissible Exposure Limit (PEL) for phosgene is $0.4 \mathrm{mg} \mathrm{m}^{-3}$ or $\sim 100 \mathrm{ppb}$ at $1 \mathrm{~atm}$ (OSHA, 1986). The PEL is the concentration of a substance to which most workers can be exposed without adverse effect averaged over a normal 8-h workday or a 40-h work week. Typically, the occupational limit would be considered to apply for workers whose job entails either production of phosgene or its use in chemical manufacturing.

In summary, these guidelines indicate a range of exposure limits, from approximately 0.1 to $100 \mathrm{ppb}$, dependent upon on the exposure duration and the population that the standard is intended to protect.

\subsubsection{Phosgene chemical characteristics}

Phosgene can undergo hydrolysis to form hydrochloric acid and carbon dioxide as shown in reaction 5-4.

$$
\mathrm{Cl}_{2} \mathrm{CO}+\mathrm{H}_{2} \mathrm{O} \rightarrow \mathrm{CO}_{2}+2 \mathrm{HCl}
$$

There is conflicting information about the speed and importance of hydrolysis for phosgene degradation. Dowideit and von Sonntag (1998) determined that phosgene formed in water from ozonolysis of a dichlorovinyl group is hydrolyzed to $\mathrm{HCl}$ so rapidly (hydrolysis rate of $\sim 9 \mathrm{~s}^{-1}$ ) that phosgene is considered to be an intermediate product. The US Agency for Toxic Substances \& Disease Registry guidelines state that phosgene is "not very water-soluble and hydrolysis is slow" (ATSDR, 2007). Kindler et al. (1995) studied the atmospheric fate of phosgene and determined that it rapidly hydrolyzes in aqueous solution, but that it is not reactive toward water vapor. In the atmosphere this means that hydrolysis could occur after wet deposition of phosgene into cloud droplets. In the aircraft cabin, this process might occur in surface-phase water on materials. 
In humid environments, water may sorb to surfaces; this process is dependent on the surface type and the relative humidity. The amount of water sorbed to surfaces can vary from negligible to a few percent of a material's weight. For example, cotton uptakes $7 \%$ of its weight at $70 \% \mathrm{RH}$ and Teflon resists sorbing water (Destaillats et al., 2006b). The relative humidity in the cabin during flight is quite low, $\sim 10-20 \% \mathrm{RH}$ (NRC, 2002). This low relative humidity arises because the source of air - ambient air at cruising altitude $(9-13 \mathrm{~km})$ - is virtually free of water; the only significant steady source of RH in the cabin is from respiration and evaporation of water from passengers' skin. Although $\mathrm{RH}$ in the cabin during flight is low, the materials are frequently exposed to elevated humidity levels while grounded and may slowly equilibrate, over the course of hours (Okubayashi, 2005). Thus, some water is likely associated with most surfaces during some portion of a typical flight. Furthermore, there is the potential for elevated local RH on the surfaces associated with passengers or in near proximity to the passengers. The effect of surface moisture on phosgene formation is unknown. Most experiments in this study were conducted under very low relative humidity conditions to minimize the possibility of hydrolysis.

\subsection{Experiments}

Based on likely levels of permethrin and ozone in the cabin, phosgene production was determined to be possible at levels of concern based on established exposure limits. The estimate of phosgene production was performed assuming that ozone and permethrin readily react, but the reaction is dependent on kinetics and byproduct generation is dependent on kinetics and yield. Laboratory experiments were conducted to develop a 
method to detect low levels of phosgene, perform a calibration using that method, and then measure phosgene formed from permethrin applied to real cabin surfaces and exposed to ozone under cabin-relevant conditions. The following sections describe experiments performed to determine the rate of ozone reaction with surface-bound permethrin and the consequent yield of phosgene under cabin-relevant conditions.

Before these experiments were performed, a preliminary set of experiments were conducted in which direct gas-phase detection of phosgene was attempted using gas chromatography with electron capture detection (GC-ECD), which is well-suited for detecting very low levels of halogenated compounds. Several experimental configurations were tested including a batch reactor (sealed bag), a high flow rate surface emissions chamber (Field and Laboratory Emission Cell, FLEC), and moderate flow rate chamber. These experiments and their results are present in an appendix, Section 5.A.

\subsubsection{Materials}

Permethrin (1000 $\mathrm{g} \mathrm{mL}^{-1}$ in methanol), phosgene (20\% in toluene), $O-(2,3,4,5,6-$ pentafluorobenzyl) hydroxylamine (PFBHA) (98+\%), acetonitrile $(\mathrm{ACN})(\geq 99.93 \%)$, 3,4-dimercaptotoluene (DMT) ( $\geq 97 \%)$ and triethylamine $\left(\mathrm{Et}_{3} \mathrm{~N}\right)(\geq 99 \%)$ were purchased from Sigma Aldrich (St. Louis, MO). Methanol high performance liquid chromatography grade (HPLC grade) was purchased from Fisher Scientific (Pittsburgh, PA). The permethrin used in this study has a reported cis:trans ratio of 65:35 (Sigma-Aldrich) and the ratio required for disinsection solutions is $25: 75$. The cis:trans ratio is not expected to affect the reactivity of the compound. 
Phosgene is a highly volatile and dangerous compound. Extreme caution was exercised in transport, transferal, and handing of this chemical. The phosgene comes from the chemical supplier in liquid form as $20 \%$ in toluene. The phosgene source was kept in a freezer, and only removed and opened for brief durations to pipette small amounts to a "stock container". The stock vial was immediately sealed with a cap that contained a septum, and was also kept in a freezer when not in use. New stock was taken approximately biweekly. The stock was transferred from the stock vial to the dilution vials by gas- and liquid-tight syringe via the septum (i.e. the phosgene would only be open to the atmosphere briefly while transferring from source to vial in a pipette and from vial to vial in a syringe). All transfers of phosgene occurred in a hood.

\subsubsection{Detection method and chemical analysis}

Phosgene was captured by bubbling phosgene-containing air through liquid in an impinger. The impinger solution contained toluene with 3,4-dimercaptotoluene (DMT), a phosgene derivatizing agent, and triethylamine $\left(\mathrm{Et}_{3} \mathrm{~N}\right)$, a catalyzing agent. The impinger arrangement is shown in Figure 5-3, and the derivatization reaction is illustrated in Figure 5-4. The derivatization product is referred to here as Phos-DMT. The phosgene detection method developed in this study was based on an earlier study by Muir et al. (2005).

Liquid injection of impinger solution was analyzed by gas chromatography (GC, Hewlett Packard 6890) with electron capture detector (ECD, Agilent 6890) and mass selective detector (MSD, Agilent 5975). In this configuration, the sample was split between the ECD and the MSD, which provided the advantage of having high sensitivity chromatographic detection of halogenated compounds, but also mass detection of 
compound fragments. With this dual-detection mode it would be possible to detect phosgene and potentially identify other products of $\mathrm{O}_{3}$-permethrin chemistry that might have formed. The MSD was operated in regular full-scan and in selective ion monitoring (SIM) mode in some cases to achieve higher sensitivity to the phosgene derivative. The ions detected in SIM mode were 182, 154, and 121, which correspond to a whole PhosDMT molecule, a Phos-DMT minus a $\mathrm{C}$ and $\mathrm{O}$, and a Phos-DMT minus a $\mathrm{C}, \mathrm{O}, \mathrm{S}$, and $\mathrm{H}$, respectively.

A DMT stock solution was made by dissolving approximately $40 \mathrm{mg}$ of solid DMT to $25 \mathrm{~mL}$ of toluene, which yielded a $10 \mathrm{mM}$ DMT stock solution. A 1:4 solution of $\mathrm{Et}_{3} \mathrm{~N}$ in toluene was also prepared, yielding a $1.8 \mathrm{M} \mathrm{Et}_{3} \mathrm{~N}$ stock solution. A $3 \mathrm{~mL}$ impinger solution was prepared by adding $0.1 \mathrm{~mL}$ DMT stock and $10 \mu \mathrm{L} \mathrm{Et}_{3} \mathrm{~N}$ stock to $2.9 \mathrm{~mL}$ toluene. DMT and $\mathrm{Et}_{3} \mathrm{~N}$ stock solutions were made weekly and impinger solution was made daily from the stocks. Impinger solution was immediately removed from the impinger after an experiment was conducted, and immediately analyzed on the GC-ECDMSD. Duplicate samples were run within 24 hours, and showed good repeatability, although the samples would begin to degrade after more than approximately one day.

\subsubsection{Calibrations}

Two phosgene-derivative calibrations were performed: (1) a "direct" derivatization calibration in which phosgene was injected into impinger solution and analyzed, and (2) a "bag" calibration in which phosgene was added to a bag and bubbled through the impinger solution as it would be in an experiment. 
Direct calibration. The direct calibration was performed by adding various dilutions of phosgene in toluene to $3 \mathrm{~mL}$ of impinger solution (that had not had air bubbled through it), and injecting $1 \mu \mathrm{L}$ samples of the solution into the GC-ECD-MSD. The number of moles of phosgene injected onto the column (in the form a phos-DMT) was calculated from the amount of phosgene added to the solution and the size of the injection; $100 \%$ derivatization was assumed. Calibration points included 0.3, 0.6, 1.25, and 2.5 pmol injected onto the column. Calibration curves for the ECD and MSD are shown in Figure 5-5. The calibration curve exhibited good linearity $\left(\mathrm{R}^{2}=0.989\right.$ for MSD in SIM mode and $\mathrm{R}^{2}=0.996$ for $\mathrm{ECD}$ ) for 0.6 to $2.5 \mathrm{pmol}$ on the column. The lowest calibration point, 0.3 pmol, could not be detected with the ECD, and was not reliably detected with the MSD. The phosgene derivative peak was "sharpest" using the MSD operated in SIM mode, so that detection method is emphasized throughout the remainder of this chapter.

Detection of phosgene-derivative by the MSD operated in SIM mode with ions 182,154 , and 121 was determined to be the optimal detection method for these experiments. The lower limit of detection was $0.6 \mathrm{pmol}$ of phosgene on the column. The study from which this method was adapted was able to achieve a lower limit of detection of 0.2 pmol on the column using MSD in SIM mode (Muir et al., 2005). One plausible reason for the higher detection limit in the current study is that the sample was split between the MSD and the ECD. One other possible reason is that some of the highly volatile phosgene evaporated, possibly during transfer, although precautions were taken to minimize evaporative losses. 
Bag calibration. The bag calibration was performed by injecting a known amount of phosgene diluted in toluene into a $100 \mathrm{~L}$ Teflon bag filled with clean, dry air. The impinger was submerged in an ice bath, and $3 \mathrm{~mL}$ of impinger solution containing 0.4 $\mathrm{mM}$ DMT and $6 \mathrm{mM} \mathrm{Et}_{3} \mathrm{~N}$ was added to the impinger. Phosgene-containing air was drawn from the bag, through the impinger, using a vacuum induced flow rate of $300 \mathrm{~mL}$ $\min ^{-1}$. After $1 \mathrm{~h}(18 \mathrm{~L}$ sampled), the experiment was stopped, the volume of the remaining impinger solution was measured (typically $2 \mathrm{~mL}$ of the $3 \mathrm{~mL}$ remained), and 1 $\mu \mathrm{L}$ samples of impinger solution were injected into the GC-ECD-MSD.

Calibration experiments were conducted for 1,2,5, and $10 \mathrm{ppb}$ of phosgene in a bag. The calibration curve (Figure 5-6) relates the phosgene-derivative peak area, which was measured with the MSD operated in SIM mode, to the phosgene level in the bag. The phosgene level was "weighted" by the injection sample size (e.g. by a factor of 1.05 if the sample size was $1.1 \mu \mathrm{L}$ instead of $1.0 \mu \mathrm{L}$ ) to correct for injecting extra (or insufficient) sample onto the column. The height of the peak corresponding to $2 \mathrm{ppb}$ was at least three times greater than the fluctuation in the baseline of the chromatogram, but the peak for 1 ppb was not. Thus, the lower limit of phosgene detection with this impinger and derivatization method was established to be $2 \mathrm{ppb}$ of phosgene. Phosgene levels of 2, 5, and $10 \mathrm{ppb}$ were detected and produced a linear calibration curve $\left(\mathrm{R}^{2}=0.94\right)$.

\subsubsection{Experimental methods}

A series of experiments were conducted with the goal of determining if phosgene could be formed from ozone reaction with surface-bound permethrin under flight-relevant conditions. Individual materials including glass and aircraft cabin surfaces (carpet, seat 
fabric, plastic wall covering) were sprayed with permethrin and exposed to ozone in a Teflon chamber. The chamber conditions simulated flight conditions including low RH and relatively high air-exchange rate. Experiments were conducted with relatively high ozone levels and permethrin surface loadings to optimize conditions for phosgene formation. The conditions of the experiments are shown in Table 5-2.

A $40 \mathrm{~L}$ cubic chamber was constructed from Teflon sheets (Figure 5-7). The bottom front of the chamber could be opened to insert a material into the chamber (not shown in the figure). The opening was sealed by folding the plastic sheets several times and clamping them. Before each experiment, the chamber was cleaned with methanol wipes, and the chamber was quenched with ozone.

Ozone-containing dry air was introduced through a nozzle in the top of the chamber, $33 \mathrm{~cm}$ above the plate or material, at a flow rate of $3.0 \mathrm{~L} \mathrm{~min}^{-1}\left(\mathrm{AER}=4.5 \mathrm{~h}^{-1}\right)$; the chamber outlet was located $8 \mathrm{~cm}$ from the front and $8 \mathrm{~cm}$ from bottom of the chamber on the right side. From the outlet flow, $1.4 \mathrm{~L} \mathrm{~min}{ }^{-1}$ was diverted to an ozone monitor, 300 $\mathrm{mL} \min ^{-1}$ was bubbled through an impinger, and the remainder was exhausted. Most experiments lasted $1 \mathrm{~h}$, although some experiments were conducted for a longer period of $4 \mathrm{~h}$.

The chamber AER $\left(4.5 \mathrm{~h}^{-1}\right)$ was approximately one-half to one-fourth the typical cabin AER (10-20 $\left.\mathrm{h}^{-1}\right)$. The ozone concentration in the supply was $150-170 \mathrm{ppb}$, except for one experiment performed with a very high ozone level of $600 \mathrm{ppb}$ and a lower AER of $1 \mathrm{~h}^{-1}$. The relative humidity was kept as low as possible, $<1 \%$, to minimize the possibility of hydrolysis. 
The materials tested were glass, carpet, plastic, and seat fabric. Borosilicate glass plates $(25 \mathrm{~cm} \times 25 \mathrm{~cm} \times 0.32 \mathrm{~cm}$, McMaster-Carr, Atlanta, GA $)$ were used as the nonreactive, smooth surface in chamber experiments and had an area of $625 \mathrm{~cm}^{2}$. Plates were cleaned with water and soap, rinsed with methanol and methylene chloride, and heated to $100{ }^{\circ} \mathrm{C}$ for at least 30 minutes between experiments. Samples of cabin surfaces (carpet, plastic, and seat fabric) were obtained from the manufacturers via Boeing. The plastic sample was from a new (unused) plastic-coated wall covering. The carpet sample was from a used runner (aisle carpet) that had been in service on an aircraft for approximately 18 months since cleaning (total duration in service was unknown). The seat fabric sample was from used seat covering that had been in service for 18 months since its last cleaning. Cabin material samples were $25 \mathrm{~cm} \times 23 \mathrm{~cm}$ for a nominal surface area of $580 \mathrm{~cm}^{2}$.

A mass of $1 \mathrm{mg}$ of permethrin (as a $1 \mathrm{~mL}$ solution of $1000 \mu \mathrm{g} \mathrm{mL}^{-1}$ in methanol) was sprayed onto the plate or cabin material using a Badger ${ }^{\circledR}$ airbrush spray gun. See Flemmer et al. (2007) for a detailed description of the spray device. The methanol was allowed to evaporate for $5 \mathrm{~min}$, and then the glass plate or material was placed in the bottom of the chamber immediately prior to starting the experiment.

This method delivered a nominal surface coverage of $1700 \mathrm{ng} \mathrm{cm}^{-2}$ (or $1600 \mathrm{ng}$ $\mathrm{cm}^{-2}$ for the glass plates since they are slightly larger), which is $\sim 17 \times$ the nominal monolayer coverage rate $\left(\sim 100 \mathrm{ng} \mathrm{cm}^{-2}\right)$ and comparable to "pre-flight" or "preembarkation" insecticide spraying guidelines $\left(\sim 700 \mathrm{ng} \mathrm{cm}^{-2}\right)$. At this level, there was a visible residue on the glass but not on cabin surfaces. The surface-to-volume ratio of the permethrin sprayed area was $1.5 \mathrm{~m}^{-1}$, which is similar to $\mathrm{S} / \mathrm{V}$ ratios for individual 
materials in the cabin. The $\mathrm{S} / \mathrm{V}$ ratio for horizontal surfaces in the cabin (which are the surfaces most likely to be coated in permethrin) is $\sim 1 \mathrm{~m}^{-1}$ and the total nominal $\mathrm{S} / \mathrm{V}$ for cabins is $4-7 \mathrm{~m}^{-1}$ (see Section 3.B.).

The efficiency of permethrin delivery was not tested, i.e. by testing the concentration of permethrin in the rinse from a plate, because an appropriate detection method was not available. However, in experiments being conducted with squalene and oleic acid at the same time as these experiments, delivery of squalene and oleic acid was confirmed by the proportionally high ozone reactivity of the sprayed plate (Wells et al., 2008).

The impinger derivatization technique described in the previous section was used to detect phosgene. However, in these experiments, air was drawn from the exhaust line of the chamber, rather than from a bag. Air from the chamber was exhausted through a Teflon tube. Very near the chamber, $300 \mathrm{ml} \mathrm{min}^{-1}$ of the exhaust flow was drawn through the impinger using a vacuum system. The impinger contained $3 \mathrm{~mL}$ of derivatizing solution in $1 \mathrm{~h}$ experiments and $6 \mathrm{~mL}$ of derivatizing solution in $4 \mathrm{~h}$ experiments. The impinger was submerged in an icebath to minimize volatilization of the toluene or derivizating agents. In each experiment, flow through the impinger was confirmed by checking for bubbling of the impinger solution.

\subsubsection{Results}

The "base case" experiment was performed with a glass plate coated in $1700 \mathrm{ng}$ $\mathrm{cm}^{-2}$ of permethrin exposed to $150 \mathrm{ppb} \mathrm{O}_{3}$ for $1 \mathrm{~h}$. No phosgene was detected in this experiment. Experiments were also conducted under the base case conditions with real 
cabin materials: carpet, seat fabric, and plastic. Variations on the base experiment conditions were performed to determine the effect of experimental parameters: a longer experiment was performed, a experiment with much higher ozone was performed (which necessitated reducing the AER because of system limitations), and a blank experiment was performed with no ozone. Phosgene formation was not detected in any of the experiments.

The strong electron-withdrawing force of chlorine atoms adjacent to the double bond in permethrin considerably slows its reaction with ozone. The presence of a surface might not greatly increase its reactivity, and the reaction rate of this molecule is just too slow for degradation to occur via this pathway on timescales important for the aircraft cabin environment. Another possibility, that could have occurred even if the reaction is slow, is that phosgene was formed but was subsequently hydrolyzed to $\mathrm{HCl}$ on the surface. Materials were exposed to lab air at $\sim 50 \% \mathrm{RH}$ for several hours before being placed in the chamber and exposed to essentially dry air. Thus, water could have sorbed to material surfaces. In either case, phosgene formation was below the detection limit with this experimental technique, $2 \mathrm{ppb}$. In the following section, this result is related back to the cabin environment using a modeling approach to estimate the expected upper limit on cabin phosgene levels given that it was not detected in these chamber experiments.

\subsection{Implications for phosgene exposure in the cabin: establishing an upper-bound on phosgene levels in aircraft cabins}

Using the ozone deposition model framework introduced in Chapter 3, the maximum reaction probability of permethrin can be estimated from the chamber 
experiments and the upper limit of phosgene formation can be predicted for the cabin environment.

In the 40-L chamber experiments, the lower limit of detection for phosgene was 2 ppb. Thus the maximum concentration of phosgene that could have been formed and remained undetected is $<2 \mathrm{ppb}$. If we assume that phosgene is being produced in the chamber, and that production is occurring at a steady-state throughout the experiments for ease of calculation, the phosgene concentration in the chamber is described by equation 5-3, reproduced here:

$$
C_{\text {phos }} \approx \frac{C_{\text {ozone }} v_{d} \frac{S_{p}}{V} Y}{\lambda}
$$

The chamber ozone level, $C_{o z o n e}$, air-exchange rate, $\lambda$, and surface-to-volume ratio, $S_{p} / V$ (where $S_{p}$ is the nominal area of the permethrin-sprayed surface and $V$ is the volume of the chamber) are known experimental conditions. The yield of phosgene, $Y$, from cypermethrin and ozone under more extreme conditions (Segal-Rosenheimer and Dubowski, 2007) can be used as an estimate: $Y=0.1$ (see Section 5.1.). Equation 5-3 can be rearranged to determine the maximum possible deposition velocity, $v_{d, \max }$, to the permethrin-coated surface given the maximum possible phosgene level, $C_{p h o s, \max }$, that could have been produced and remained undetected, as shown in equation 5-5.

$$
v_{d, \max } \approx \frac{C_{\text {phos } \max } \lambda}{C_{\text {ozone }} \frac{S_{h}}{V} Y}
$$

For the conditions of the "base case" experiment (\#4, Table 5-2), the maximum deposition velocity of ozone to a permethrin-covered surface is: 


$$
v_{d, \max } \approx \frac{2 \mathrm{ppb} \times 4.5 \mathrm{~h}^{-1}}{150 \mathrm{ppb} \times 1.5 \mathrm{~m}^{-1} \times 0.1}=0.4 \mathrm{~m} \mathrm{~h}^{-1}=0.01 \mathrm{~cm} \mathrm{~s}^{-1} \text {. }
$$

Phosgene formation was not detected under even more conducive conditions in the high ozone, low AER experiment, which indicates an even lower maximum deposition velocity. Under the condition of that experiment (\#7, Table 5-2), the maximum deposition velocity is:

$$
v_{d, \max } \approx \frac{2 \mathrm{ppb} \times 1.5 \mathrm{~h}^{-1}}{600 \mathrm{ppb} \times 1.5 \mathrm{~m}^{-1} \times 0.1}=0.03 \mathrm{~m} \mathrm{~h}^{-1}=0.001 \mathrm{~cm} \mathrm{~s}^{-1}
$$

In Chapter 3, the method for extrapolating deposition velocities measured in chambers to other environments was explained. That process involves determining the reaction probability of the material (or as in this case, chemical residue) and the flow conditions in both environments. Reaction probabilities of cabin materials (and most indoor surfaces) are in the transition or transport-limited deposition regimes, and thus deposition velocity depends on air flow conditions, parameterized by the friction velocity (see Figure 5-8). However, in the present case, the deposition velocity is so low that it does not depend on flow conditions. The kinetically-limited deposition velocity is related to the reaction probability by equation 5-6 (equation 34 from Cano-Ruiz et al., 1993).

$$
v_{d} \approx \frac{\gamma\langle v\rangle}{4}
$$

This equation can be rearranged to determine the maximum reaction probability of ozone with a permethrin-covered surface:

$$
\gamma_{\max } \approx \frac{4 \times 0.001 \mathrm{~cm} \mathrm{~s}^{-1}}{3.6 \times 10^{4} \mathrm{~cm} \mathrm{~s}^{-1}}=10^{-7}
$$


As shown in Figure 5-8, the reaction probability is indeed well into the kinetically limited regime.

Reaction probability is independent of flow conditions as are kinetically-limited deposition velocities. Consequently, the deposition velocity measured in the chamber can be used directly in modeling conditions in the cabin. The steady-state approximation is employed here to determine an approximate upper bound on the phosgene level in the cabin. Substituting typical values for the cabin environment without an ozone converter (20 ppb $\mathrm{O}_{3}$ (Bhanghar et al., 2008); $\sim \mathrm{m}^{-1}$ for horizontal $\mathrm{S} / \mathrm{V}$ ratio; and an air-exchange rate of $10 \mathrm{~h}^{-1}$ ), one obtains an estimate for the maximum phosgene concentration in the cabin of $0.02 \mu \mathrm{g} \mathrm{m}^{-3}$.

$$
C_{\text {phos }}<\frac{20 \mathrm{ppb} \times 0.03 \mathrm{~m} \mathrm{~h}^{-1} \times 1 \mathrm{~m}^{-1} \times 0.1}{10 \mathrm{~h}^{-1}}=0.007 \mathrm{ppb}=0.02 \mu \mathrm{g} \mathrm{m}^{-3}
$$

Under more extreme conditions the ozone level might be 200 ppb (Bhangar et al., 2008; Spengler et al., 2004) and the surface-to-volume ratio for material coated in permethrin might be higher, for example, $\sim 5 \mathrm{~m}^{-1}$. In this case the maximum phosgene level might be $1 \mu \mathrm{g} \mathrm{m}^{-3}, \sim 50 \times$ higher than the estimate for typical conditions. In these calculations mol fraction units (ppb) were converted to concentration units $\left(\mu \mathrm{g} \mathrm{m}^{-3}\right)$ using cabin-relevant pressure and temperature of $0.8 \mathrm{~atm}$ and $295 \mathrm{~K}$; under these conditions, air has a molar concentration of $33 \mathrm{~mol} \mathrm{~m}^{-3}$.

$$
C_{p h o s}<\frac{200 \mathrm{ppb} \times 0.03 \mathrm{~m} \mathrm{~h}^{-1} \times 5 \mathrm{~m}^{-1} \times 0.1}{10 \mathrm{~h}^{-1}}=0.3 \mathrm{ppb}=1 \mu \mathrm{g} \mathrm{m}^{-3}
$$

The experiments in this study indicate that ozone-permethrin reactions in the cabin could be responsible for - at maximum - a phosgene level of $1 \mu \mathrm{g} \mathrm{m}^{-3}$. This study supports the conclusion that the OSHA limit of $400 \mu \mathrm{g} \mathrm{m}^{-3}$ would certainly not be 
exceeded in the cabin. Phosgene formation also appears not likely to exceed the OEHHA

limit of $4 \mu \mathrm{g} \mathrm{m}^{-3}$. Recall that acute exposure at or below this guideline is considered to be adequate to protect against mild adverse health effects (OEHHA, 1999); thus, this seems to be the most appropriate guideline for evaluating aircraft cabin exposures. The most stringent phosgene exposure guideline is the USEPA's RfC of $0.3 \mu \mathrm{g} \mathrm{m}^{-3}$, which is designed to protect even sensitive populations from adverse effects owing to chronic lowlevel exposures. Sensitive persons are unlikely to fly frequently, so this guideline may be overly stringent for guiding a health-risk assessment for aircraft cabins.

\subsection{Permethrin, ozone, and phosgene in indoor environments}

This study has focused on detecting phosgene formation under typical aircraft cabin conditions because permethrin is the commonly used aircraft residual insecticide. However, permethrin is also the most frequently used pyrethoid in the U.S. (ATSDR, 2003), and its use as a home and garden insecticide may be increasing because of restrictions on the organophosphate insecticides diazanon and chlorpyrifos (Bekarian et al., 2006). It is approximated that 2 million pounds of permethrin are applied in the United States each year, $\sim 30 \%$ by homeowners, $\sim 40 \%$ by professionals in nonagricultural settings, $3 \%$ in mosquito abatement programs, and the remainder in agricultural settings (EPA, 2006). Permethrin is also registered for use in lice shampoo, and in this form it is applied directly to children's heads (EPA, 2006).

Permethrin and other pyrethroids with the dichlorovinyl functional group could be precursors for phosgene formation in indoor environments. The relevant differences between aircraft cabins and building environments are that building air-exchange rates 
are much lower, the relative humidity in buildings is usually higher, and the surface area per volume is somewhat lower than in the cabin environment. Ozone is present in buildings and homes because of ventilation with ozone-containing outdoor air or because of an indoor source such as an ozone-generating 'air purifier' or certain office equipment. Methods of pyrethroid application are similar in the aircraft cabin as in buildings: spray cans, foggers, and pressurized sprayers are used for application indoors as well. Matoba et al. (1998) used the "crack and crevice" method to apply phenothrin in a chamber experiment that simulated spraying in an apartment $\left(23 \mathrm{~m}^{3}\right.$ chamber volume, AER $=1.5$ $\left.\mathrm{h}^{-1}, 60 \% \mathrm{RH}\right)$. The average concentration on the floor (the only horizontal surface) was $2200 \mathrm{ng} \mathrm{cm}^{-2}$ after spraying. Concentrations on the ceiling and walls were an order of magnitude lower. As shown in Figure 5-2, these surface concentrations of pyrethroids after "crack and crevice" treatment are similar to those found in aircraft cabins.

Members of sensitive populations (very young, very old, and ill people) are not likely to spend much time in flight, but do spend a significant amount of time in their homes and other buildings (such as hospitals). If phosgene were formed at low levels in residential environments owing to ozone reactions with chlorinated insecticides, it could be a cause for concern. One can substitute typical values for residential and commercial environments into equation 5-3 to approximate the maximum possible phosgene levels expected in buildings based on the experiments reported in this chapter. A typical indoor ozone concentration is $\sim 10 \mathrm{ppb}$ and the surface-to-volume ratio for permethrin sprayed surfaces is likely to be $\sim 1 \mathrm{~m}^{-1}$. Air exchange rates in buildings are about an order of magnitude lower than in aircraft cabins $\sim 1 \mathrm{~h}^{-1}$ versus $\sim 10 \mathrm{~h}^{-1}$. The estimated maximum 
deposition velocity for buildings is the same as for the chamber because the deposition velocity was determined to be kinetically-limited. Therefore,

$$
C_{\text {phos }}<\frac{10 \mathrm{ppb} \times 0.03 \mathrm{~m} \mathrm{~h}^{-1} \times 1 \mathrm{~m}^{-1} \times 0.1}{1 \mathrm{~h}^{-1}}=0.03 \mathrm{ppb}=0.1 \mu \mathrm{g} \mathrm{m}{ }^{-3}
$$

The upper bound phosgene level under typical conditions for residential and commercial environments is approximately an order of magnitude greater than the aircraft cabin owing to an order of magnitude lower air-exchange rate. The value calculated for typical conditions is of similar magnitude to the reference concentration of

$0.3 \mu \mathrm{g} \mathrm{m}^{-3}$ for chronic inhalation exposure recommended by the EPA (EPA, 2005).

Ozone-initiated oxidation does not appear to be a dominant degradation pathway for permethrin. It is not known if or how permethrin degrades indoors and what are the degradation products. In soil, permethrin is degraded by aerobic and anaerobic soil degradation or photolysis. Photolysis is a degradation pathway in water as well, but permethrin has a very high affinity for soil owing to its low water solubility, low volatility, and high octanol-water partition coefficient (CDPR, 2003). Very few studies of permethrin degradation have been published. One older study indicates that photolysis causes ester cleavage, breaking of the molecule at the ester group ( $\mathrm{R}-\mathrm{C}(\mathrm{O})-\mathrm{R})$, and that the degradation products include 3-phenoxybenzyl alcohol and dichlorovinyl acid (Holmstead et al., 1978).

\subsection{Conclusions}

Phosgene was identified as a potential reaction product of ozone and permethrin, the most common aircraft cabin residual insecticide. A literature search was conducted to determine that surface levels of permethrin in cabins that have been recently treated 
(within weeks) are 10-1000 $\mathrm{ng} \mathrm{cm}^{-2}$ or higher. Based on likely levels of permethrin and ozone in the cabin, it was deemed conceivable that phosgene production could occur in planes at levels of potential concern with regard to health guidelines.

A method for detecting phosgene at low levels was developed. It involved derivatizing phosgene by bubbling air through an impinger containing liquid toluene and the derivatizing agent dimercaptotoluene. Impinger solutions were analyzed in SIM mode by a GC-MSD. Two types of calibrations were performed for the phosgene derivative (phos-DMT), direct derivatization and derivatization in an experiment configuration, and the lower limits of detection under these experimental conditions were $0.6 \mathrm{pmol}$ on the column and $2 \mathrm{ppb}$ in the gas phase, respectively.

Experiments were conducted in a continuously ventilated $40 \mathrm{~L}$ Teflon chamber. Permethrin was sprayed on glass and cabin materials (carpet, seat fabric, and plastic) at a surface concentration of $1700 \mathrm{ng} \mathrm{cm}^{-2}$, roughly equal to 17 monolayers of permethrin. Materials were exposed to ozone at cabin-relevant conditions $\left(150 \mathrm{ppb} \mathrm{O}_{3}\right.$, an air exchange rate of $4.5 \mathrm{~h}^{-1}$, and $<1 \%$ relative humidity), and at other conditions thought to be more conducive to phosgene formation $\left(\sim 600 \mathrm{ppb} \mathrm{O}_{3}\right.$, an air exchange rate of $1.5 \mathrm{~h}^{-1}$, and $<1 \%$ relative humidity). Phosgene was not detected in any of the experiments.

The reason that phosgene formation was below detection limits, or did not occur, appears to be that the chlorine atoms neighboring the double bond in permethrin are strongly electron-withdrawing, which greatly reduces the tendency of ozone to react at this site. It was proposed that $\mathrm{OH}$, feasibly formed in ozone reactions with other surface constituents of cabin materials, might still react rapidly enough with permethrin to form phosgene. However, phosgene was not detected even in experiments with aircraft cabin 
materials proven to be reactive with ozone (as opposed to glass which does significantly consume ozone), which are experiments where $\mathrm{OH}$ would likely be formed.

Results from the experiments were extrapolated to the aircraft cabin environment using a simple model of ozone transport and uptake. It was determined that phosgene formation, if it occurs in the cabin, is not likely to produce levels exceeding the OEHHA guideline for mild adverse effects owing to acute exposure ( $4 \mu \mathrm{g} \mathrm{m}^{-3}$ or $\left.1 \mathrm{ppb}\right)$, even under extreme cabin ozone conditions. This is an important finding for the health of passengers and especially for flight crew members who spend a significant amount of their time in aircraft cabins.

These experiments did not conclusively prove that phosgene is not formed, rather that the formation rate is below a certain level. However, based on these experiments, it does not appear likely that ozone-initiated oxidation or $\mathrm{OH}-$ related oxidation of permethrin is a major route of degradation for permethrin in indoor spaces, nor a major source of phosgene. 
Table 5-1: Effect of chlorine substitution on ozone reaction rate $\left(\mathrm{cm}^{3} \mathrm{molec}^{-1} \mathrm{~s}^{-1}\right)$ with simple alkenes.

\begin{tabular}{|c|c|c|c|}
\hline Compound & Name & AOPwin $^{a}$ & Measured \\
\hline & Ethane & $1.8 \times 10^{-18}$ & $1.5 \times 10^{-18 b}$ \\
\hline & Chloroethene & $2.5 \times 10^{-19}$ & $4.3 \times 10^{-20 b}$ \\
\hline & 1,1-dichloroethene & $3.5 \times 10^{-20}$ & $6.2 \times 10^{-21 c}$ \\
\hline
\end{tabular}

\footnotetext{
${ }^{\mathrm{a}}$ Estimated ozone reaction rate (Meylan and Howard, 1993)

${ }^{\mathrm{b}}$ Ljubic and Sabljic (2002)

${ }^{c}$ Avzianova and Ariya (2002)
} 
Table 5-2: Conditions of permethrin oxidation experiments with impinger derivatization detection technique. ${ }^{\mathrm{a}}$

\begin{tabular}{ccccccc}
\hline Exp. \# & Material & $\begin{array}{c}\text { Exhaust } \\
\text { Ozone } \\
(\mathrm{ppb})^{\mathrm{b}}\end{array}$ & $\begin{array}{c}\text { Permethrin } \\
\text { coverage } \\
\left(\mathrm{ng} \mathrm{cm}^{-2}\right)\end{array}$ & $\begin{array}{c}\text { Experiment } \\
\text { Duration } \\
(\mathrm{h})\end{array}$ & $\begin{array}{c}\text { AER } \\
\left(\mathrm{h}^{-1}\right)\end{array}$ & RH \\
\hline \hline 1 & Glass (long, blank) & 170 & 0 & 4 & 4.5 & $<1 \%$ \\
2 & Glass (long) & 170 & 1700 & 4 & 4.5 & $<1 \%$ \\
3 & Glass (blank) & 170 & 0 & 1 & 4.5 & $<1 \%$ \\
4 & Glass (base case) & 150 & 1700 & 1 & 5 & $<1 \%$ \\
5 & Carpet & $\sim 90$ & 1700 & 1 & 4.5 & $<1 \%$ \\
6 & Wall covering & 160 & 1700 & 1 & 4.5 & $<1 \%$ \\
7 & Seat fabric & $\sim 80$ & 1700 & 1 & 4.5 & $<1 \%$ \\
8 & Glass (high ozone) & $\sim 600$ & 1700 & 1 & 1.5 & $<1 \%$ \\
\hline
\end{tabular}

${ }^{\mathrm{a}}$ The base-case and deviations from the base-case conditions are highlighted.

${ }^{\mathrm{b}}$ The ozone supply level was $170 \mathrm{ppb}$ in all experiments except experiment $\# 5$ in which the supply level was $150 \mathrm{ppb}$ and experiment $\# 8$ in which the supply level was $\sim 600 \mathrm{ppb}$. 
(a)

Phenothrin<smiles>CC(C)=C[C@@H]1C(C(=O)OCc2cccc(Oc3ccccc3)c2)C1(C)C</smiles>

(b)<smiles>CCC1C(C(=O)OCc2cccc(Oc3ccccc3)c2)C1(C)C</smiles>

Permethrin<smiles>CC1(C)[C@H](C=C(Cl)Cl)[C@H]1C(=O)OCc1cccc(Oc2ccccc2)c1</smiles>

Figure 5-1: (a) Chemical structures of the common cabin insecticides phenothrin and permethrin. The difference between the two compounds is that the two terminal methyl groups $\left(-\mathrm{CH}_{3}\right)$ on phenothrin are replaced by chlorine atoms on the permethrin molecule. (b) Permethrin (chemical formula: $\mathrm{C}_{21} \mathrm{H}_{20} \mathrm{O}_{3} \mathrm{Cl}_{2}$; molecular weight $=391 \mathrm{~g} / \mathrm{mol}$; CAS No. 52645-53-1; vapor pressure at $25^{\circ} \mathrm{C}=4.4 \times 10^{-10} \mathrm{~atm}$ ) might react with ozone at the double bond to produce phosgene, a highly toxic substance (chemical formula $=\mathrm{CCl}_{2} \mathrm{O}$; molecular weight $=98.9 \mathrm{~g} / \mathrm{mol}$; CAS No. 75-44-5). 


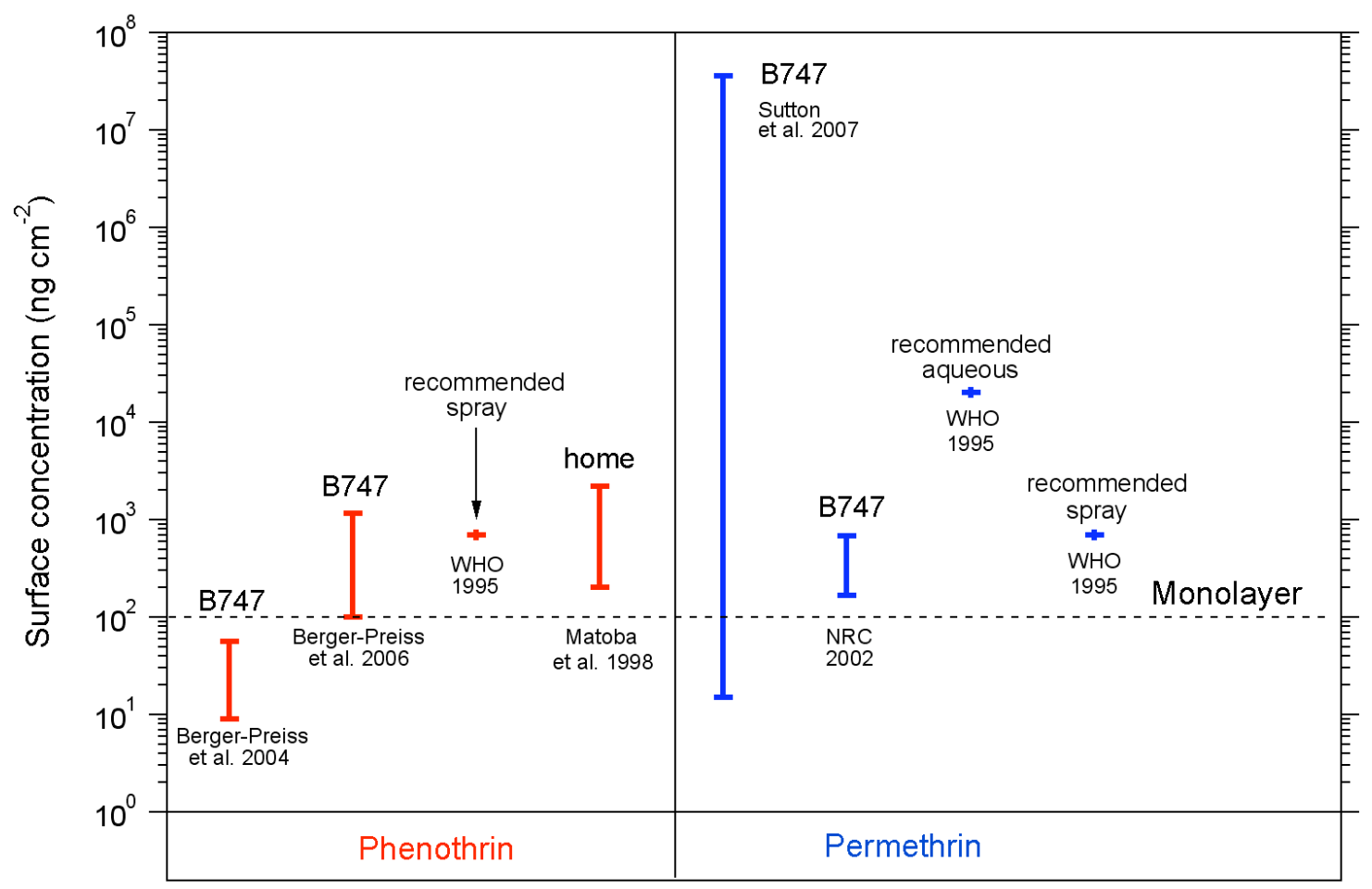

Figure 5-2: Surface concentrations of phenothrin and permethrin measured in four cabin studies (in B747s) and one chamber study that replicated typical conditions in a home. Also shown are estimated surface concentrations that result from the spray and aqueous delivery procedures recommended by WHO for cabin disinsection. The dashed line indicates the estimated surface concentration that corresponds to monolayer coverage of permethrin on a smooth surface. 


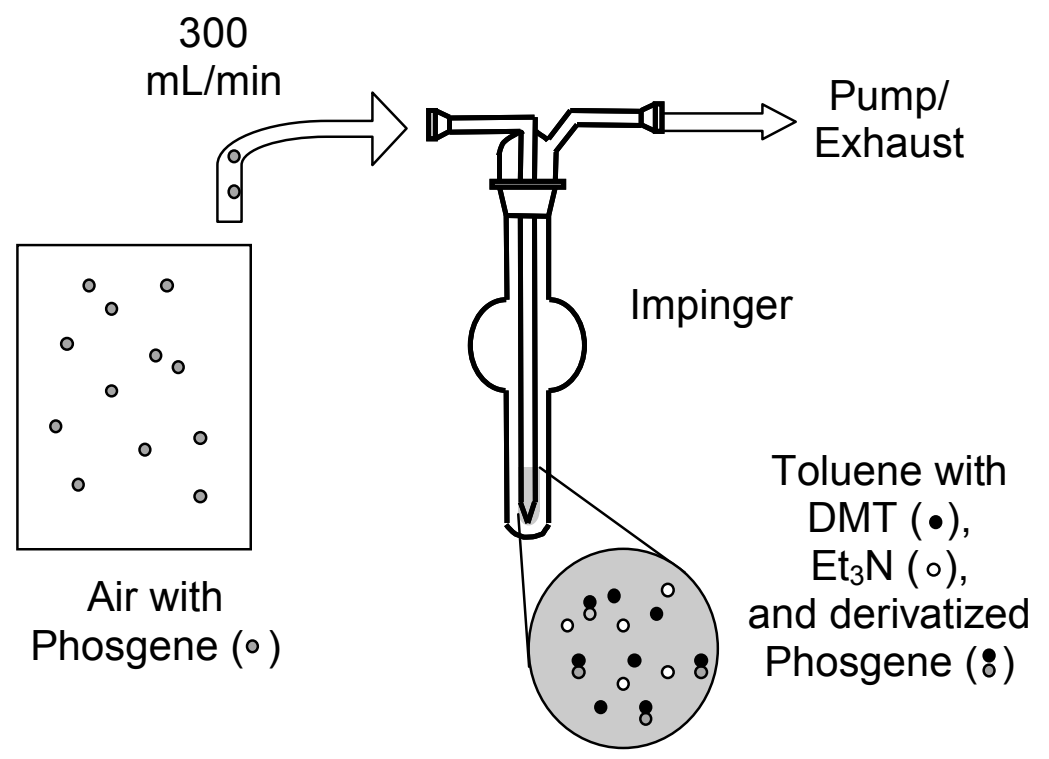

Figure 5-3: Schematic of the derivatization technique for phosgene. Gaseous phosgene is bubbled through a liquid solution of toluene containing the derivatizing agent dimercaptotoluene (DMT), and a catalyzing agent triethylamine $\left(\mathrm{Et}_{3} \mathrm{~N}\right)$. 


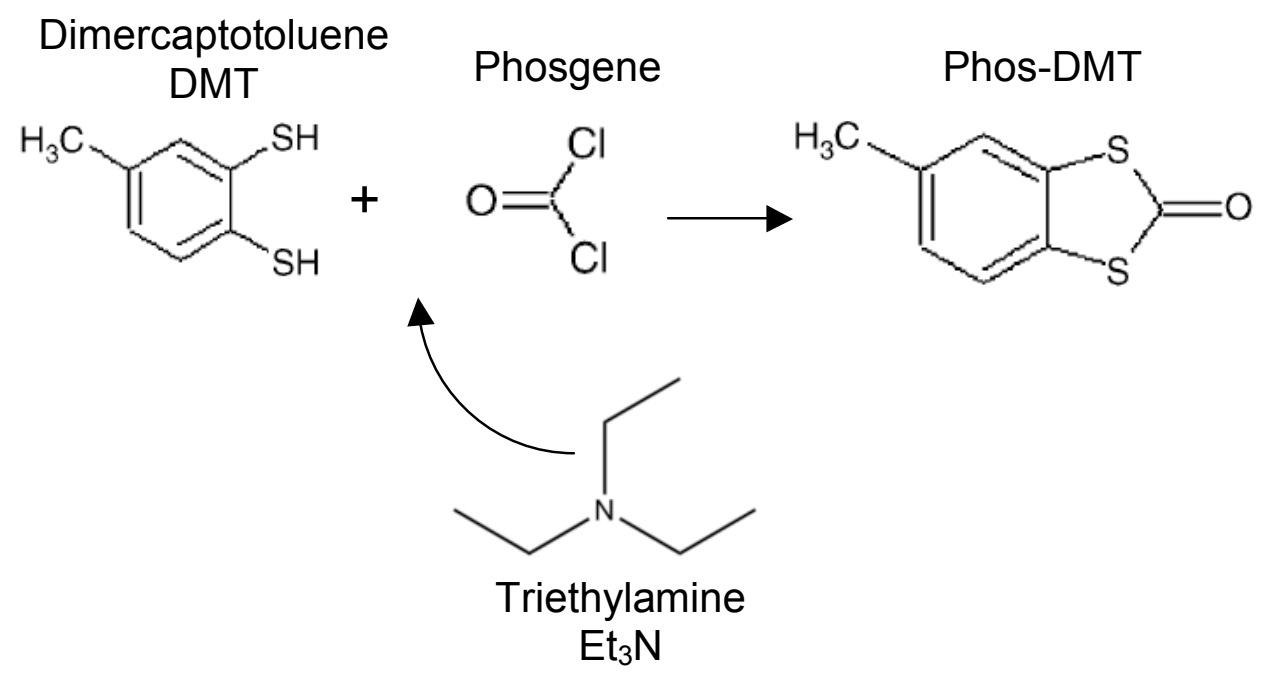

Figure 5-4: Derivatization reaction for phosgene with dimercaptotoluene and triethylamine based on the work of Muir et al. (2005). 


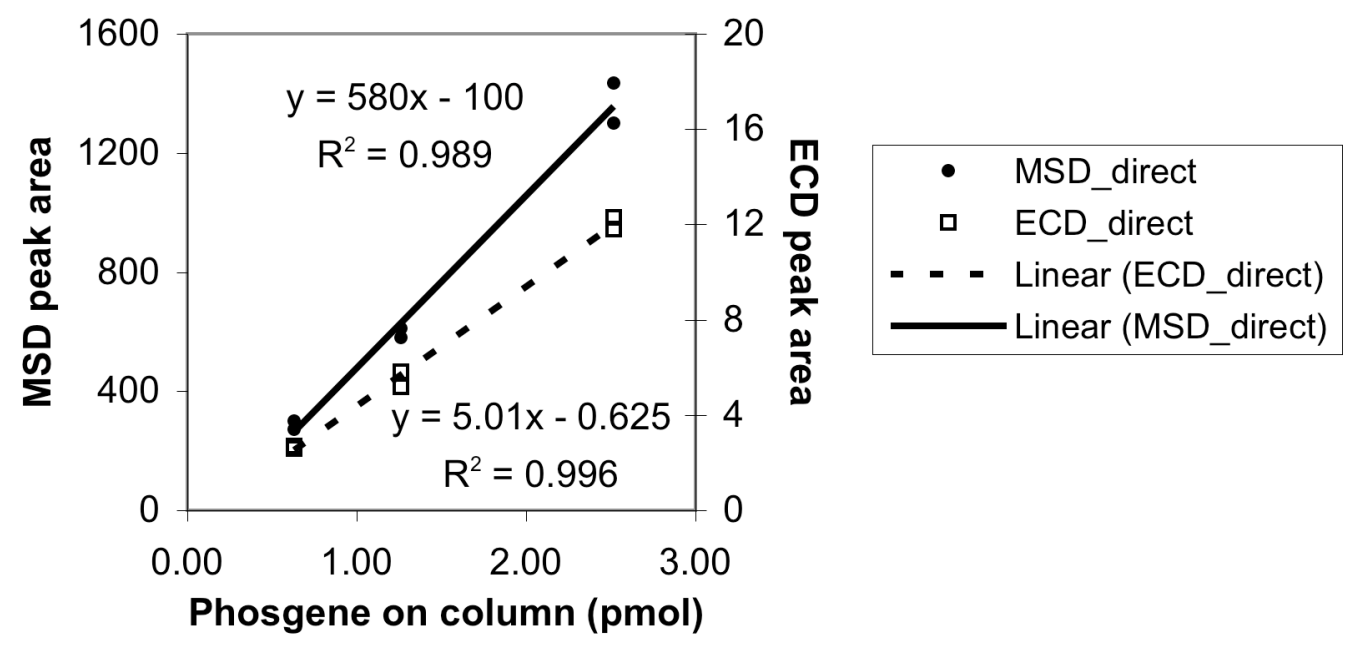

Figure 5-5: Calibration curves for direct liquid phosgene derivatization. The MSD was operated in SIM mode using $\mathrm{m} / \mathrm{z} 121,154$, and 182 . Note that the ECD peak area is shown on the right axis. The amount of phosgene injected onto the column was calculated from the stated concentration of the phosgene ( $20 \%$ in toluene by weight). 


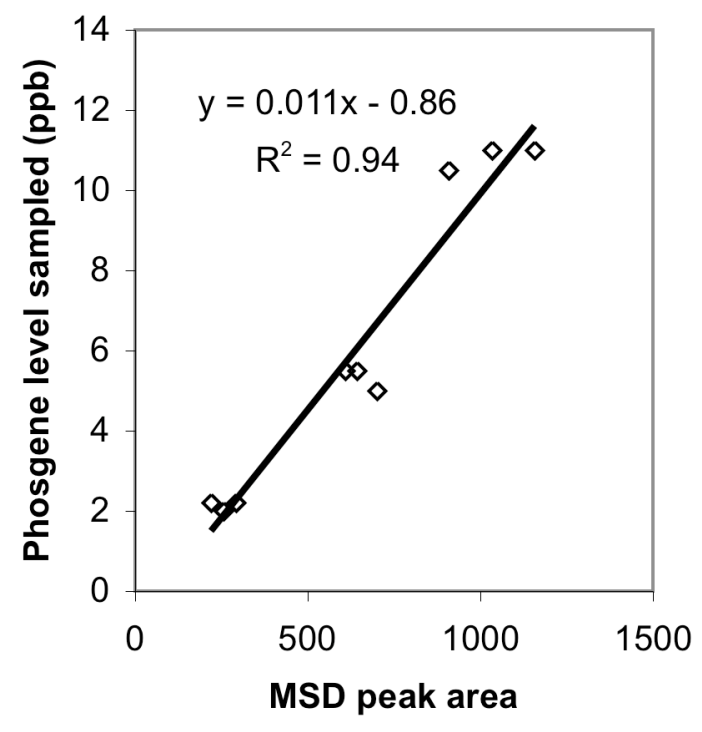

Figure 5-6: MSD calibration curve for sampling phosgene in air. Known levels of gasphase phosgene $(2,5$, and $10 \mathrm{ppb})$ from a bag were bubbled though an impinger containing the derivatizing agents 3,4-dimercaptotoluene and triethylamine in liquid toluene. The phosgene level in the bag was weighted by the injection sample size. The MSD was operated in SIM mode using $\mathrm{m} / \mathrm{z}$ 121, 154, and 182. 


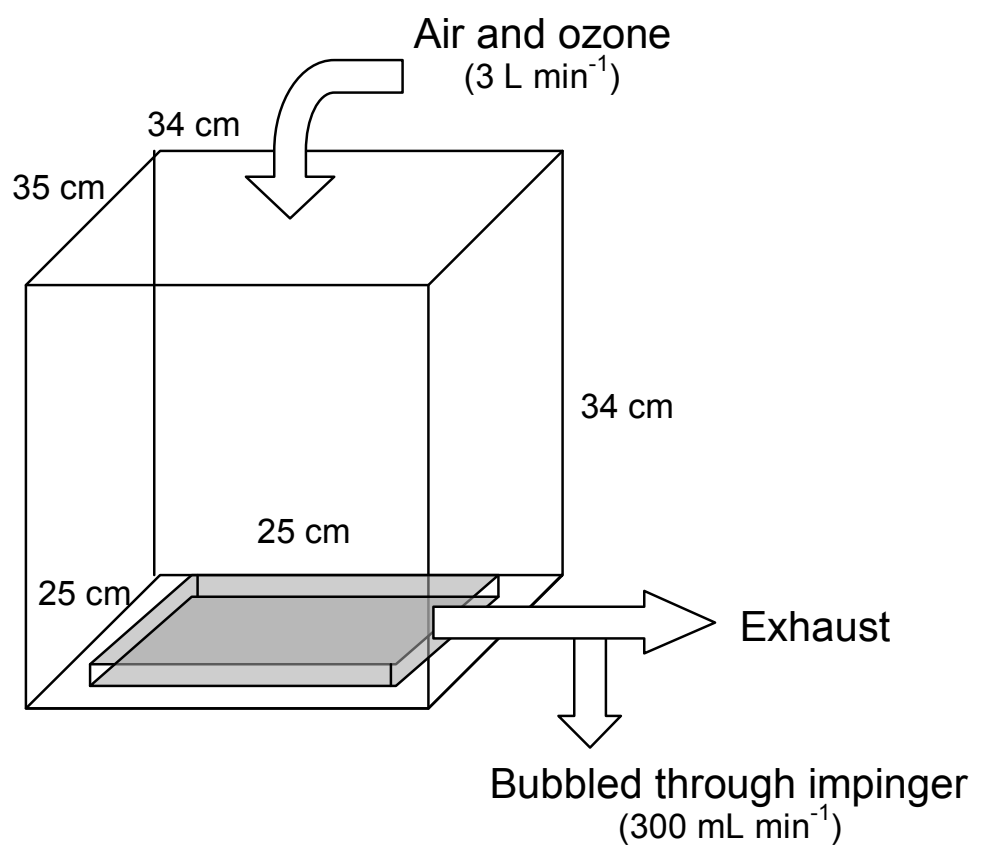

Figure 5-7: 40 L Teflon CFMR chamber for continuous flow, surface-bound permethrin oxidation experiments. 


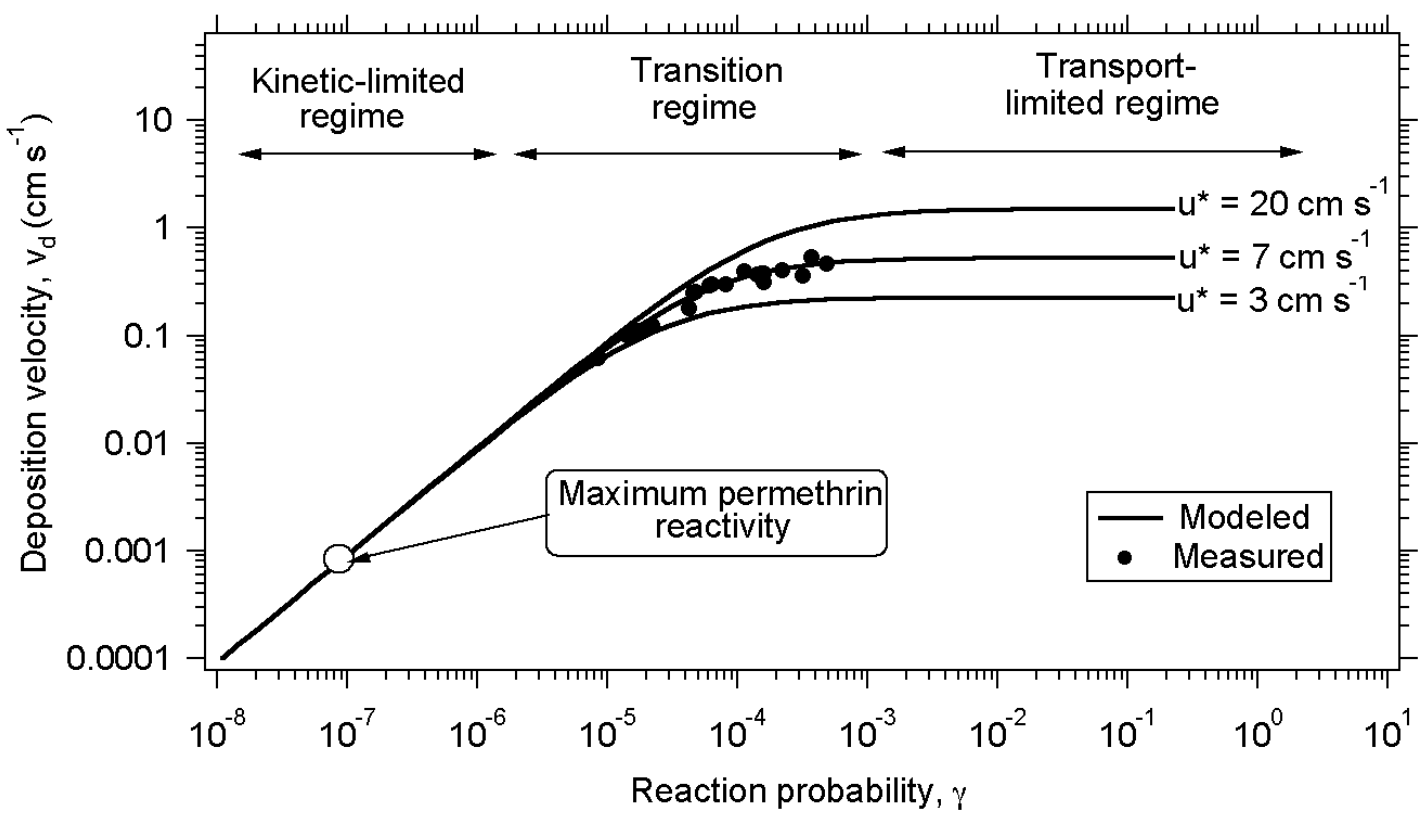

Figure 5-8: Deposition model for reactive gas uptake on indoor surfaces developed by Morrison and Nazaroff (2002b) with kinetic, transition, and transport-limited deposition regimes shown. The measured data points are from chamber studies of ozone deposition to cabin materials (see Chapter 3 of this dissertation). Based on the experiments presented in this chapter, permethrin has reaction probability less than $10^{-7}$. 


\section{A. Preliminary experiments to detect phosgene from ozone reaction with permethrin}

A series of preliminary experiments was performed to determine if phosgene formation from the ozone-permethrin reaction was detectable using a direct sampling

method. Experiments involved permethrin on a smooth nonreactive surface such as glass or Teflon and were performed using a three reactor types: a Field and Laboratory Emission Cell (FLEC), a batch reactor, and a continuously mixed flow reactor (CMFR). A description of the experimental configurations, the detection methods, and the experimental results follows. Phosgene was not detected from the reaction of permethrin and ozone in any of the chamber experiments. In addition, attempts to derivatize phosgene with $O-(2,3,4,5,6$-pentafluorobenzyl) hydroxylamine (PFBHA) are described. Derivatization with this detection method was unsuccessful.

\section{A.1. Chemical analysis}

Samples were collected in Teflon bags at the exhaust of the reaction chambers and analyzed by GC-ECD. Gas-phase analysis was made possible by using a concentrator, Entech (Simi Valley, CA), which consists of a series of Tenax and glass traps just before thermal injection onto the GC-ECD. The GC (Hewlett Packard 6890) oven was initially held at $35^{\circ} \mathrm{C}$ for $5 \mathrm{~min}$ and then ramped at $20^{\circ} \mathrm{C} \min ^{-1}$ to $180{ }^{\circ} \mathrm{C}$ where it was held for $5 \mathrm{~min}$. The ECD (Agilent Model 6890) was operated at $300{ }^{\circ} \mathrm{C}$ with 60 $\mathrm{mL} \min ^{-1}$ of $\mathrm{N}_{2}$ make-up flow. The triplicate runs for each sample yielded consistent results. Since the phosgene stock was dissolved in toluene and diluted with methanol, sequential ECD tests were performed with (1) just methanol, (2) methanol plus toluene, 
and (3) methanol plus toluene and phosgene. The phosgene peak was clearly identified with a retention time of $\sim 5.05 \mathrm{~min}$.

The chemicals and glass plates used in these experiments are described in Section 5.4.4. Sampling bags were produced on-site by heat-sealing Teflon film and adding Teflon sampling fixtures. Sampling bags were cleaned by at least 10 cycles of automated filling with clean, dry air and exhausting. Sampling bags had a dedicated use, i.e. a bag was only used for clean air or ozonated air. A sampling bag was retired after phosgene had been injected into it.

\section{A.2. FLEC experiments}

Experiments were conducted using a Field and Laboratory Emissions Cell (FLEC) (CHEMATEC Aps., Denmark). The FLEC is portable stainless steel chamber that is set directly upon a building surface and used to test emissions (Wolkoff, 1995). The FLEC apparatus and experimental configuration is shown in Figure 5A-1. The FLEC was designed to introduce a radial flow field of uniform velocity over a defined surface area with a diameter of $15 \mathrm{~cm}$, although the actual flow field may not be uniform (Zhang and Niu, 2003). Air is introduced through a $1 \mathrm{~mm}$ slit around the circumference of the FLEC and flows toward the center of the FLEC where the air is exhausted and can be sampled for surface emissions. The FLEC has a small clearance above the surface $(\sim 1$ $\mathrm{mm}$ at the minimum and $\sim 20 \mathrm{~mm}$ at maximum). The FLEC was coupled to an automated control system (FACS) (Flemmer et al., 2007) that maintained and recorded environmental conditions of air being introduced into the chamber such as air-flow rate, ozone concentration, and relative humidity. 
The experimental conditions were set at 10\% RH, $300 \mathrm{~mL} \mathrm{~min}^{-1}$ FLEC flow, and $200 \mathrm{ppb}$ supply ozone. The air-exchange rate (AER) at this flow rate is $\sim 500 \mathrm{~h}^{-1}$. To establish a background for compounds detectable by ECD, experiments were conducted with a clean glass plate + ozone, with a plate sprayed with methanol only + ozone, and with a plate sprayed with methanol and permethrin + clean air (no ozone).

A circular template with a diameter of $149 \mathrm{~mm}$ was employed to give an accurate and consistent spray area and to ensure that the FLEC was positioned precisely over the sprayed area (the FLEC flow field diameter is $150 \mathrm{~mm}$ ). After a plate was sprayed it was left for 5 min to allow the methanol to evaporate. The plate was then exposed to ozonecontaining air or clean air, depending on the desired experimental conditions.

For experiments with permethrin, $1 \mathrm{~mL}$ of $4 \times 10^{-5} \mathrm{M}$ permethrin in methanol was sprayed on the glass plate. This application rate yielded a permethrin surface coverage of $100 \mathrm{ng} \mathrm{cm}^{-2}$, which corresponds to roughly monolayer coverage. Experiments lasted one hour and the FLEC exhaust for the entire $1 \mathrm{~h}$ was collected in a Teflon bag for analysis on ECD, except in some experiments where the FLEC exhaust was diverted to a Thermo 49i photometric $\mathrm{O}_{3}$ monitor (ThermoScientific, Franklin, Massachusetts) in order to determine ozone reaction kinetics.

In the FLEC experiments, ozone consumption was consistently below detection. This finding was determined by comparing the profile of the exhaust ozone concentration for a plate sprayed with methanol only to plates sprayed with $\sim 1 \times$ and $\sim 2.5 \times$ the estimated monolayer concentration of permethrin (Figure 5A-2a). The total amount of permethrin on the plate was $\sim 40 \mathrm{nmol}$ for $1 \times$ monolayer coverage and $\sim 100 \mathrm{nmol}$ for $2.5 \times$ monolayer coverage. The molar rate of ozone flowing through the FLEC was 2.5 
nmol $\min ^{-1}$ for 60 min giving a total of $150 \mathrm{nmol}$ of ozone throughout the course of the experiment, which is moderately in excess of the permethrin. However, at any given moment the amount of ozone in the FLEC was $0.3 \mathrm{nmol}$ (corresponding to $200 \mathrm{ppb}$ ozone level and FLEC volume of $35 \mathrm{~mL}$ ); the permethrin was present in excess, so the ozone concentration at the exhaust would have been significantly lowered, at least initially, if surface-bound permethrin was highly reactive with ozone. In another study, a monolayer of oleic acid (one unsaturation) and squalene (six unsaturations) each exhibited high ozone consumption under similar test conditions (Wells et al., 2008).

GC-ECD chromatograms for two FLEC experiments, one without ozone and one with ozone, are shown in Figure 5A-3b. The characteristic spike for phosgene occurs at $\sim 5.05 \mathrm{~min}$, but no peak eluted at that retention time. Thus, phosgene was not detected in the ozone (or clean air) FLEC experiment(s). There was a unique peak at $\sim 4.65 \mathrm{~min}$ for the ozone experiment. At the time of these experiments, the compound responsible for the peak could not be identified (there was no mass selective detector at that time). Phosgene was injected into sampling bags after an experiment and run again on the ECD to confirm the position of the phosgene peak. Despite precautions used in preparing sampling bags, it is possible that the peak at $4.65 \mathrm{~min}$ is an oxidation product associated ozone reactivity in the bag. However, this seems unlikely as the peak was not seen in any other experiments with the same type of bag. It could also be a true oxidation product of permethrin. Is it feasible that a byproduct be formed at detectable levels if no ozone was observed to be consumed? Reaction of an undetectably small amount of ozone could form ppb levels of byproducts. If $1 \%$ of ozone molecules were transformed into a byproduct, then the concentration of byproducts would be 2 ppb for $200 \mathrm{ppb} \mathrm{O}_{3}$. 
Since the AER rate in the FLEC was high and the expected reaction rate for permethrin is low, the conditions of the FLEC may not be well-suited to detect the ozonepermethrin reaction. A batch reactor experiment was then performed to determine if phosgene could be detected with a longer residence time for the reagents to react.

\section{A.3. Batch reactor experiments}

A batch reactor experiment was performed with a $25 \mathrm{~L}$ Teflon bag. First, the bag was with filled with $12 \mathrm{~L}$ of clean air at $10 \% \mathrm{RH}$. Next, $100 \mu \mathrm{g}$ of permethrin $(100 \mu \mathrm{L}$ of $1000 \mu \mathrm{g} \mathrm{mL}^{-1}$ in methanol) was injected into the bag and "background" samples were taken using the GC-ECD. The bag was then filled with $12 \mathrm{~L}$ of air with $500 \mathrm{ppb}$ ozone and $10 \% \mathrm{RH}$, giving the bag an initial ozone concentration of $250 \mathrm{ppb}$ ozone. Since the vapor pressure of permethrin is quite low $\left(4.4 \times 10^{-10} \mathrm{~atm}\right)$, the vast majority of the compound is expected to be partitioned to the surface of the bag. However, permethrin partitioning between air and Teflon could behave quite differently than partitioning over the pure liquid. The area of the bag was $\sim 0.6 \mathrm{~m}^{2}$, and so the surface concentration of permethrin was $\sim 20 \mathrm{ng} \mathrm{cm}^{2}$ if all of the permethrin was sorbed to the surface and evenly distibuted. The initial number of moles of ozone and permethrin in the bag were equal. Samples were taken from the bag using GC-ECD immediately after ozone addition, $\sim 3 \mathrm{~h}$ after ozone addition, and $\sim 60 \mathrm{~h}$ after ozone addition. The bag was then spiked with $\sim 200$ ppb phosgene and resampled to confirm the appearance of the phosgene peak.

The GC-ECD runs are shown in Figure 5A-3; triplicate samples were taken at each of the five times but only one trace is shown for each time because triplicate runs 
were nearly identical. The trace for the phosgene-spiked sample is projected onto the scale at half size so that the details of all traces can be seen.

The sample taken from the bag at $60 \mathrm{~h}$ showed only a small peak at $5.03 \mathrm{~min}$ (approximately where phosgene is expected to appear), which may indicate a small amount of phosgene formation. Perhaps the more interesting result was the growth of the peaks at 4.6 and $4.7 \mathrm{~min}$. The first peak corresponds to the unidentified peak from the FLEC ozone experiment. (The retention times of the batch reactor runs are shifted $\sim 0.05$ min backward from the FLEC runs so that the unidentified peak at 4.65 min in the FLEC experiment corresponds to the unidentified peak at $4.60 \mathrm{~min}$ in the batch reactor experiment.) Again, the analysis technique employed was not capable of identifying this product. The peak did not appear in subsequent experiments where a GC-ECD-MSD was employed, and so the compound was not identified.

\section{A.4. CMFR reactor experiments}

In this series of experiments, permethrin was exposed to ozone under conditions that more closely simulated the cabin environment in terms of surface-to-volume ratio and air exchange rate. A $40 \mathrm{~L}$ cubic chamber was constructed from Teflon sheets (Figure 5-7). The bottom front of the chamber could be opened to insert a material into the chamber (not shown in the figure). The opening was sealed by folding the plastic sheets several times and clamping them. The chamber was operated as a continuously mixed flow reactor (CMFR).

Three experiments were performed with the CMFR: (1) ozone, no plate; (2) $1 \mathrm{~mL}$ methanol sprayed onto a glass plate, and (3) $510 \mu \mathrm{g}$ of permethrin in $1 \mathrm{~mL}$ of methanol 
sprayed onto a glass plate, which corresponds to a permethrin surface coverage of 2880 $\mathrm{ng} \mathrm{cm}{ }^{-2}$ and $\sim 30 \times$ the estimated monolayer concentration. Before each experiment the chamber was cleaned with methanol wipes, and the chamber was quenched with $275 \mathrm{ppb}$ ozone. Plates were sprayed as described for the FLEC experiments, using the circle template to ensure a consistent spraying area of $175 \mathrm{~cm}^{2}$, although the entire plate (625 $\mathrm{cm}^{2}$ ) was exposed in the chamber. The methanol was allowed to evaporate for $5 \mathrm{~min}$, and then the plate was placed in the bottom of the chamber just prior to starting the experiment. The $\mathrm{S} / \mathrm{V}$ ratio of the permethrin sprayed area was $0.5 \mathrm{~m}^{-1}$; the $\mathrm{S} / \mathrm{V}$ ratio for horizontal surfaces in an aircraft cabin is $\sim 1 \mathrm{~m}^{-1}$ and the total nominal $\mathrm{S} / \mathrm{V}$ for cabins is 4-7 $\mathrm{m}^{-1}$ (see Section 3.B.).

Air with $275 \mathrm{ppb}$ ozone and $13 \% \mathrm{RH}$ was introduced through a nozzle in the top of the chamber $33 \mathrm{~cm}$ above the plate at a flow rate of $2.95 \mathrm{~L} \mathrm{~min}^{-1}\left(\mathrm{AER}=4.4 \mathrm{~h}^{-1}\right)$; the chamber outlet was located $8 \mathrm{~cm}$ from the front and $8 \mathrm{~cm}$ from bottom of the chamber on the right side. From the outlet flow, $1.4 \mathrm{~L} \mathrm{~min}^{-1}$ was diverted to an ozone monitor, $0.3 \mathrm{~L}$ $\min ^{-1}$ was collected in a bag for analysis by GC-ECD, and the remainder was exhausted. In the CMFR experiments, the chamber AER $\left(4.4 \mathrm{~h}^{-1}\right)$ was approximately onehalf to one-fourth the typical cabin AER $\left(10-20 \mathrm{~h}^{-1}\right)$. Based on a comparison of the temporal profiles of the chamber ozone concentration as measured at the exhaust, a glass plate with $30 \times$ the estimated monolayer concentration of permethrin was no more reactive than a clean plate. Also, the exhaust ozone concentration profile for the plate with permethrin followed the curve for an ideal CMFR with step input of $250 \mathrm{ppb}$ ozone (Figure 5A-4a). The difference between the input and steady state ozone concentration ( 275 to $250 \mathrm{ppb}$ ) is due to imperfect sealing of the chamber. No phosgene was measured 
in the exhaust collected from the chamber as shown in Figure 5A-4b. The maximum concentration of phosgene formed would be $\sim 40 \mathrm{ppb}$ assuming that every molecule of permethrin applied was converted to phosgene, and this would have required that $20 \%$ of the total ozone that flowed through the chamber over the $4 \mathrm{~h}$ would have been consumed.

\section{A.5. PFBHA derivatization}

In addition to directly sampling phosgene from the gas phase, attempts were made to derivatize phosgene in the gas and surface phases. The derivatizing agent employed in this preliminary attempt to derivatize phosgene was $O-(2,3,4,5,6$-pentafluorobenzyl) hydroxylamine (PFBHA). Three types of phosgene derivatization experiments were conducted. First, phosgene ( $20 \%$ in toluene) was mixed directly with PFBHA in a methanol solution. Second, exhaust from an ozone-permethrin FLEC experiment was bubbled through an impinger containing methanol and PFBHA. Third, subsequent to ozone exposure in a FLEC experiment, the permethrin (and possible surface-bound byproducts) were rinsed from the plate using methanol and then PFBHA was added. The amount of derivatizing agent added was $100 \mu \mathrm{L}$ of $10 \mathrm{mM}$ PFBHA (in acetonitrile) to every $1 \mathrm{~mL}$ of methanol solution. In each of the three cases, $2 \mathrm{~mL}$ of the PFBHA/methanol solution were left overnight to form derivatives, then dehydrated with ultra high purity $\mathrm{N}_{2}$ and reconstituted with $100 \mu \mathrm{L}$ of methanol. The derivatized samples were analyzed on a Varian (Palo Alto, CA) 3800/Saturn 2000 gas chromatography/mass spectrometry (GC-MS) system operated in dual electron impact (EI) and chemical ionization (CI) modes. See Flemmer et al. (2007) for details of the chemical analysis 
method. Rinse effluent that was not derivatized was analyzed by direct injection in the GC-ECD system.

No phosgene derivative was detected in these preliminary phosgene derivative experiments. In the case of the impinger and surface rinse experiments, this might be because no phosgene was formed. However, attempts to directly mix phosgene with PFBHA in a methanol solution also did not form derivatives. Thus, it is clear that this derivatization method is flawed. There are two likely reasons. First, the chemical mechanism of carbonyl derivatization is based on the attraction of the nitrogen (hydroxylamine) group of the PFBHA to the electron-rich double bond of the carbonyl. The same principle that affected permethrin-ozone reactivity affects phosgene's capacity for derivatization: the two adjacent chlorine atoms greatly reduce the electron density of the double bond making phosgene less likely to derivatize. Second, during the course of our experiments, it was discovered that phosgene undergoes decomposition in methanol. A phosgene standard that had been diluted with methanol was injected in the ECD over time. The methanol-based phosgene standard significantly degraded over the course of three-weeks but had not decomposed completely. Methanol should likely not be used as a solvent for phosgene derivatization chemistry. 
(a)

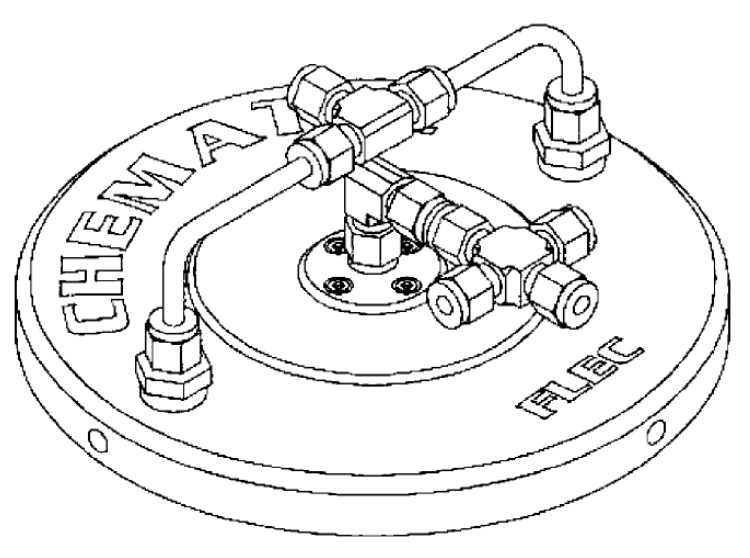

(b)

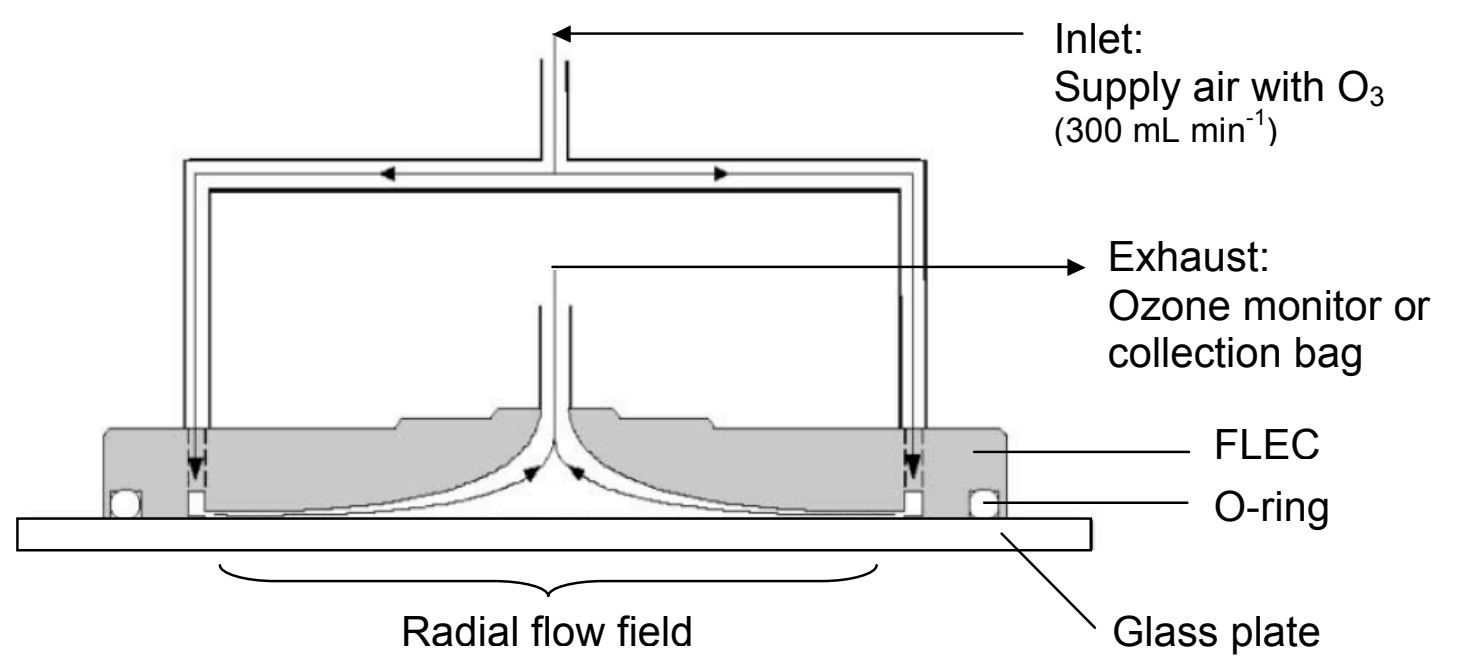

Figure 5A-1: Schematics of (a) Field and Laboratory Emission Cell (FLEC) and (b) FLEC experimental configuration for permethrin reaction experiments. Figures were adapted from Zhang and Niu (2003). A template with a diameter of $149 \mathrm{~mm}$ was used to ensure permethrin was sprayed with in the diameter of the FLEC flow field $150 \mathrm{~mm}$ ). Diagrams are not drawn to scale. 
(a)

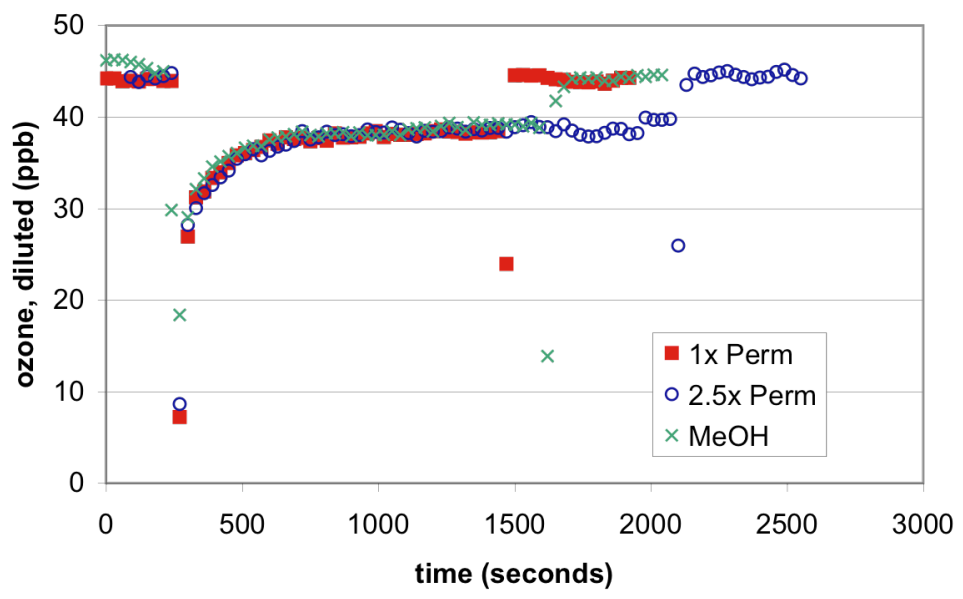

(b)

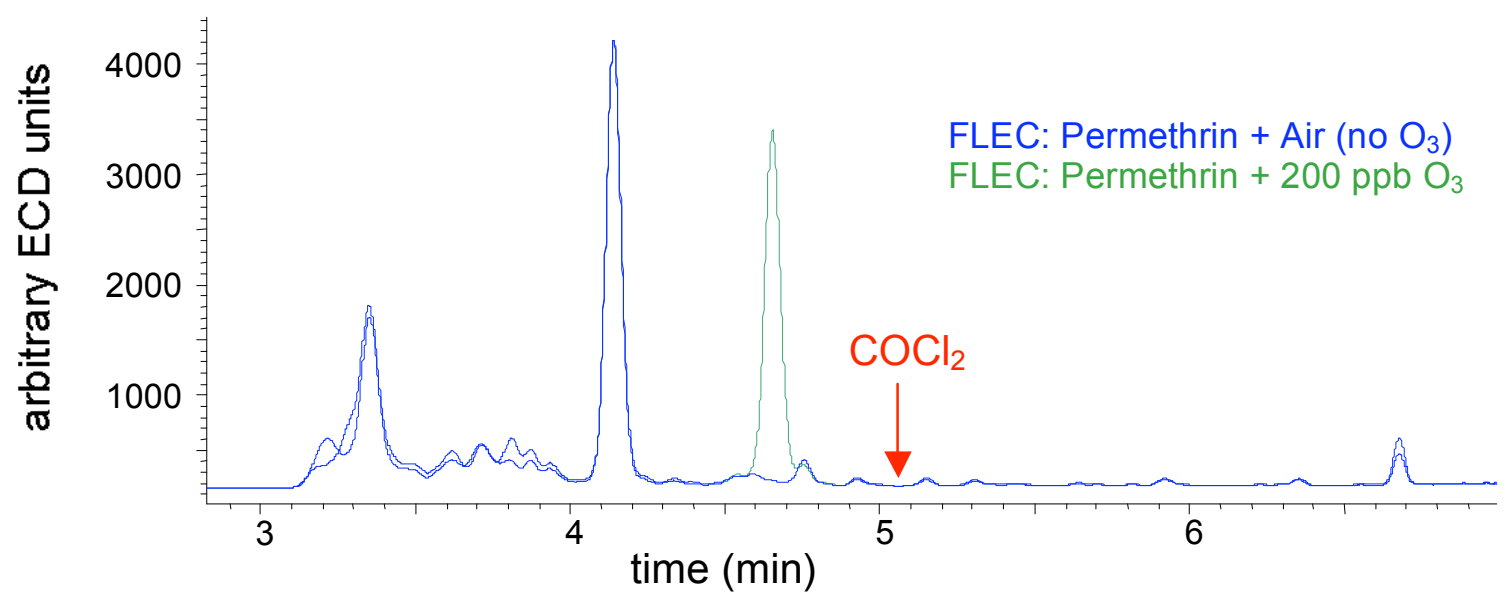

Figure 5A-2: Ozone reactivity and byproducts of permethrin as tested using a FLEC. The FLEC experiments were performed for $1 \mathrm{~h}$ at $10 \% \mathrm{RH}$. a $300 \mathrm{~mL} \mathrm{~min}^{-1}$ flow rate, and with 200 ppb ozone (when used). Figure 5A-2a shows the ozone concentration measured at the exhaust of the chamber for experiments where the glass was sprayed with just methanol $(\mathrm{MeOH}), 100 \mathrm{ng} \mathrm{cm}^{-2}(1 \times)$ and $250 \mathrm{ng} \mathrm{cm}^{-2}(2.5 \mathrm{x})$ of permethrin. Figure $5 \mathrm{~A}-2 \mathrm{~b}$ shows GC-ECD chromatograms for experiments where a glass plate was sprayed with a $100 \mathrm{ng} \mathrm{cm}^{-2}$ of permethrin and exposed to air with and without ozone. The retention time where phosgene would have eluted is indicated with an arrow. The compound responsible for the peak at $4.65 \mathrm{~min}$ for the ozone+permethrin experiment was not identified. 


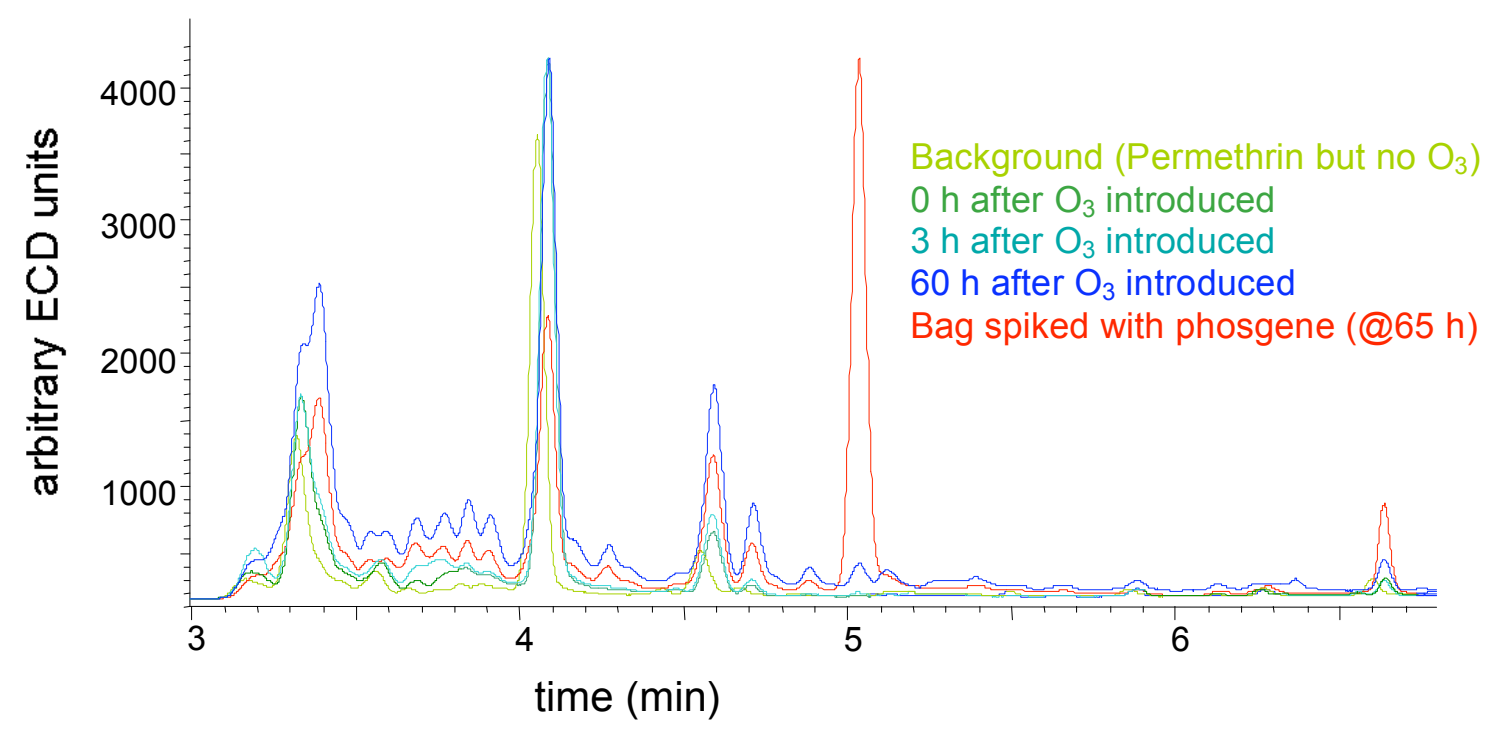

Figure 5A-3: GC-ECD chromatograms taken from the batch reactor. The batch reactor was a $25 \mathrm{~L}$ Teflon bag injected with $100 \mu \mathrm{g}$ permethrin and filled with $250 \mathrm{ppb}$ ozone. 
(a)

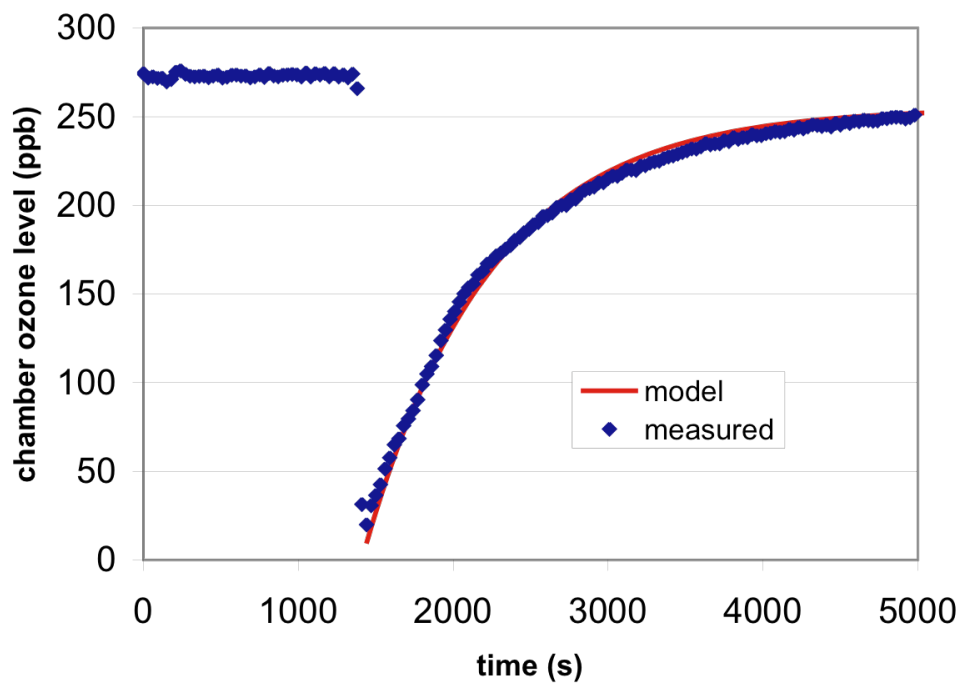

(b)

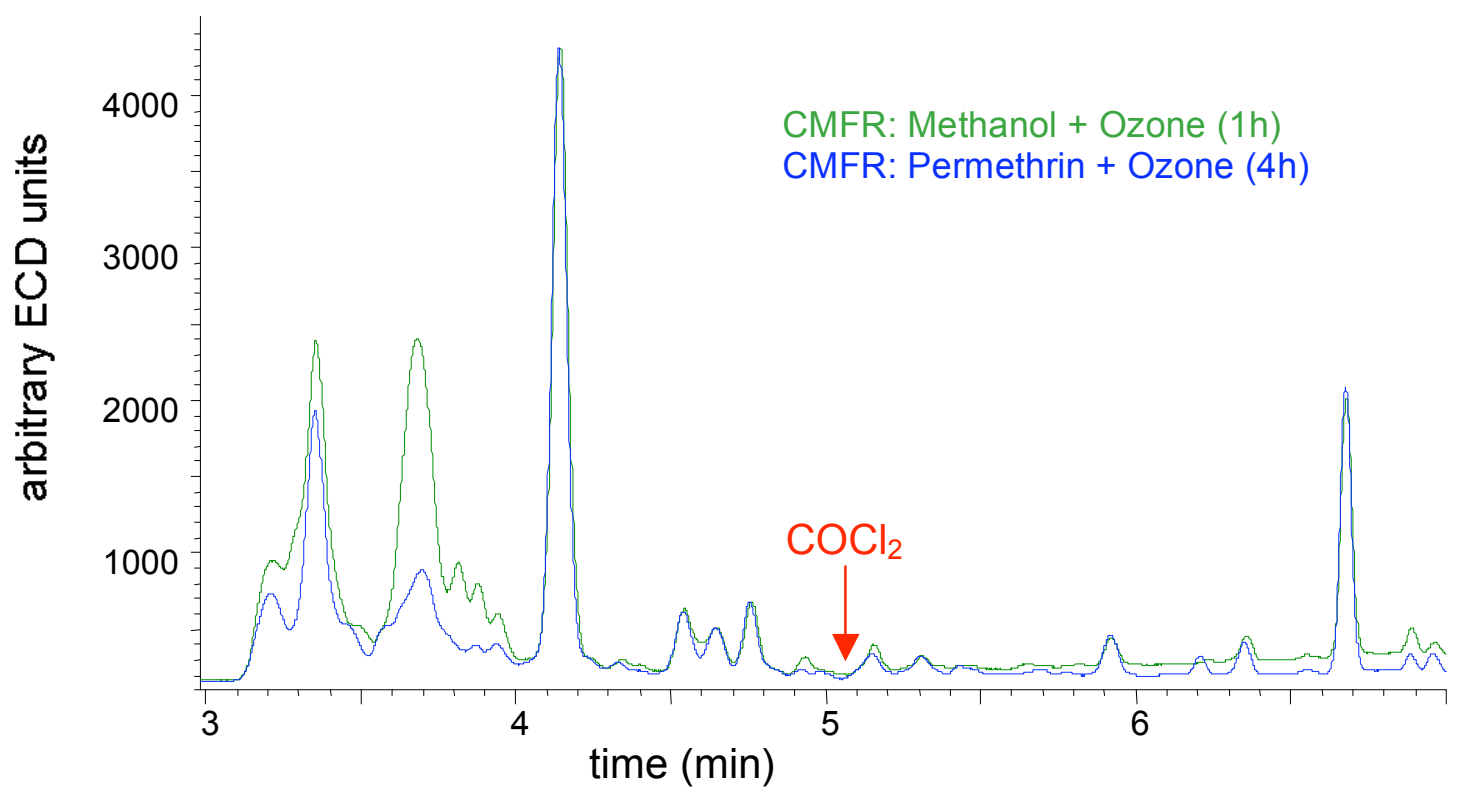

Figure 5A-4: Ozone reactivity and byproducts of permethrin as tested using a CMFR. The CMFR was $40 \mathrm{~L}$ in volume and experiments were performed with $2.95 \mathrm{~L} \mathrm{~min}^{-1}, 13 \%$ $\mathrm{RH}, 250 \mathrm{ppb}$ ozone, $2880 \mathrm{ng} \mathrm{cm}^{-2}$ permethrin (when used). Figure 5A-4a shows the ozone concentration measured at the exhaust of the chamber. The chamber was empty until 1400s when the permethrin-sprayed plate was added to the chamber. Also shown is the modeled ozone concentration for the chamber if no sinks were present. Figure 5A-4b shows the GC-ECD chromatograms for integrated samples during methanol (clean plate) + ozone and permethrin + ozone experiments. The retention time where phosgene would have eluted is indicated with an arrow 


\section{Summary and recommendations of future research}

In the indoor environment, ozone readily reacts with many gas-phase and surfacebound compounds. These reactions lower the indoor ozone level but produce secondary oxidation byproducts that may be more harmful or irritating than ozone. Thus, ozone, a ubiquitous ambient pollutant, transforms organic compounds into other pollutants in the indoor environment where we spend the majority of our time.

In this dissertation, the effects of ozone reactions on indoor air quality were explored. Reactions in the gas phase and on surfaces were investigated for conditions in building environments, such as homes and offices, and in the aircraft cabin environment. Another important aspect of ozone chemistry that was explored was reactions with human skin oil and the resulting potential for formation of oxidation byproducts in immediate proximity to the breathing zone.

This work highlights the connection between the outdoor and the indoor environment and emphasizes the need to consider this connection for the sake of health and comfort. This work also identifies a source of pollution exposure that is not accounted for when measurements of ambient pollution are used to estimate population exposures.

There are two main practical implications of this dissertation. First, these studies show that the only way to minimize exposure to reaction byproducts is to minimize exposure to ozone. This means that ozone generators should not be operated in the presence of humans, efforts should be made to control ozone entering indoor spaces, and outdoor ozone levels should be minimized through pollution control. Second, the studies 
conducted provide evidence that it is possible to gain a good understanding of the governing factors of complex systems with well-designed experiments and models. The different categories of indoor environments vary widely (e.g. the aircraft cabin has important differences from a house), and even within categories there are large variations. However, this complexity can be distilled and tested in relatively convenient experiments to provide good insight for indoor pollution exposure issues.

In the following sections, I summarize the four major research topics of my dissertation and offer recommendations for future research based on my findings.

\subsection{Secondary organic aerosol from indoor ozone-terpene reactions}

In Chapter 2, I analyzed secondary organic aerosol (SOA) data from a series of small-chamber experiments in which terpene-rich vapors from household products were combined with ozone under conditions analogous to product use indoors. Reagents were introduced into a continuously ventilated $198 \mathrm{~L}$ chamber at steady rates. Consistently, at the time of ozone introduction, nucleation occurred similar to events observed in the outdoor ambient atmosphere in both polluted urban and cleaner forested areas. The initial nucleation burst and growth was followed by a period in which approximately stable particle levels were established, reflecting a balance between new particle formation, condensational growth, and removal by ventilation. Airborne particles were measured with a scanning mobility particle sizer (SMPS, 10-400 nm) in every experiment and with an optical particle counter $(\mathrm{OPC}, 0.1-2.0 \mu \mathrm{m})$ in a subset. Parameters for a three-mode lognormal fit to the size distribution at steady state were determined for each experiment. At the outset of this work, the experiments described here were the first to measure the 
range of particles sizes required to observe SOA formation and growth events from ozone-terpene reactions under indoor conditions.

Analysis of the SOA data confirmed that nucleation occurs and growth processes are affected by environmental factors such as air-exchange rate and seed particle concentration. Increasing the supply ozone level increased the steady-state mass concentration and yield of SOA from each product tested. Decreasing the air-exchange rate increased the yield. The steady-state fine-particle mass concentration $\left(\mathrm{PM}_{1.1}\right)$ ranged from 10 to $>300 \mu \mathrm{g} \mathrm{m}^{-3}$ and yields (SOA mass generated per unit VOC mass consumed) ranged from $5 \%$ to $37 \%$. Steady-state nucleation rates and SOA mass formation rates were $\sim 10 \mathrm{~cm}^{-3} \mathrm{~s}^{-1}$ and $\sim 10 \mu \mathrm{g} \mathrm{m}^{-3} \mathrm{~min}^{-1}$, respectively. The particle size distribution data analyzed here is from well-controlled laboratory experiments, which allowed for isolation of controlling factors and made detailed analysis of particle dynamics possible. Particle measurements have been made in large chamber experiments and field studies, and particle behavior in real environments is similar to the behavior exhibited in these chamber experiments, so conclusions drawn from the small chamber experiments appear to be applicable to those environments.

Indoor sources of particles should be examined because of the proximity and duration of exposure to indoor sources of pollution. More experiments in real environments with realistic use of terpene-containing products would help to elucidate the factors that affect particle dynamics and resulting human exposures. Methods to determine particle composition have been developed and applied widely in outdoor environments, but many of these methods have yet to be employed in indoor settings. Traditional integrated sampling methods employing filters could be used to determine 
indoor SOA composition. However, new in-situ instruments have been developed that are capable of detecting hourly, speciated organic composition of particles (Williams et al., 2006) and use of this type of instrument in indoor environments would reveal not only composition, but temporal patterns of exposure to indoor SOA. Studies of particle exposure will be useful inputs for particle health impact studies.

Although the addition of $\mathrm{NO}_{\mathrm{X}}$ did not appear to affect nucleation and condensation processes in these experiments, the composition of particles could have been affected. Emerging information about the effects of $\mathrm{NO}_{\mathrm{X}}$ on atmospheric SOA chemistry (e.g. Presto et al., 2005) and indoor studies of $\mathrm{NO}_{\mathrm{X}}$ chemistry would be useful for exploring the affects of indoor $\mathrm{NO}_{\mathrm{X}}$ on particle health effects.

\subsection{Ozone consumption and byproduct formation of cabin surfaces}

In Chapter 3, I described measurements of ozone consumption by, and byproduct formation from, individual surface materials commonly found in aircraft cabins. Two series of small-chamber experiments were conducted at flight-relevant conditions; most experiments were conducted at low relative humidity $(10 \%)$ and high air-exchange rate $\left(20 \mathrm{~h}^{-1}\right)$. New and used cabin materials (seat fabric, carpet, and plastic) and laundered and worn clothing fabrics (cotton, polyester, and wool) were studied. I measured ozone deposition to many material samples, and I measured ozone uptake and primary and secondary emissions of volatile organic compounds (VOCs) from a subset of samples.

These experiments provided data about ozone consumption, specifically deposition velocities and reaction probabilities, on fixed indoor surfaces under aircraft cabin-relevant conditions, and data about ozone reactions with skin-oil laden surfaces. 
Deposition velocities ranged from 0.06 to $0.54 \mathrm{~cm} \mathrm{~s}^{-1}$ and reaction probabilities ranged from $8 \times 10^{-6}$ to $1 \times 10^{-4}$. These experiments also established emissions profiles for surface materials and skin oil residue under cabin relevant conditions. Emissions of VOCs were higher with ozone than without ozone in every case. The most commonly detected secondary emissions were $\mathrm{C}_{1}$ through $\mathrm{C}_{10}$ saturated aldehydes and the squalene oxidation products 6-methyl-5-hepten-2-one and acetone. For the compounds measured, summed VOC emission rates in the presence of 55-128 ppb (residual level) ozone were $1.0-8.9 \mu \mathrm{mol} \mathrm{h}^{-1} \mathrm{~m}^{-2}$. Total byproduct yield ranged from 0.07 to 0.24 moles of product volatilized per mole of ozone consumed. Results from these experiments were used to estimate the relative contributions of different materials to ozone deposition and byproduct emissions in a typical aircraft cabin. The dominant contributors to both were clothing fabric - owing to reaction with skin oil residue - followed by seat fabric. I estimated that $60-80 \%$ of ozone that enters the cabin is consumed in reactions with surface. These reactions generate volatile byproducts of potential concern for the health and comfort of passengers and crew. Total airborne reaction byproduct levels were predicted to be similar to the cabin ozone level.

Cabin air quality studies have highlighted the effect that human occupants have on ozone consumption and byproduct concentrations. Chapter 3 and other studies of ozone reactions with squalene, fatty acids, and skin-oil chemistry provide ample evidence that ozone reactions on human skin and with human-skin oil on other surfaces can be a major sink of ozone and an important source of ozone-initiated byproducts (Wisthaler et al., 2005; Tamás et al., 2006; Weschler et al., 2007; Wells et al., 2008). However, the chemical mechanism of ozone reactions with squalene and prevalent fatty acids and their 
reaction byproducts has not been thoroughly investigated, and this is an area of potential importance owing to the prevalence of skin oil in indoor environments, especially near the breathing zone of occupants. "Near-head" or "personal cloud" chemistry is an emerging area of study that determines ozone and byproduct gradients in close proximity to the breathing zone of a person who is exposed to ozone (Corsi et al., 2007). In addition to skin oil, ozone may react with products applied to the hair or skin, such as shampoo or lotions that contain fatty acids or terpenes (Pandrangi and Morrison, 2008). Studies are needed to more fully explore the effects of environmental conditions and personal habits on reactive pollutant chemistry near the breathing zone.

\subsection{Modeling ozone reactions with residue covered surfaces}

In Chapter 4, a model was developed for predicting ozone consumption by and gas-phase byproduct formation from an ozone-reactive residue that partially covers a surface in an indoor environment. The equations developed here build on an established model framework of ozone transport and uptake (Cano-Ruiz et al., 1993; Morrison and Nazaroff, 2002b). The allows ozone level to be held constant (i.e., ozone reactions with the surface of interest do not affect the overall indoor ozone concentration), or to depend on ozone uptake by the surface of interest (as would be the case in a chamber experiments where only one reactive surface is present and the chamber ozone concentration depends on that material's reactivity). Model simulations were run that explore the effects of factors such as reactivity of residue, reactivity of base material, air flow conditions, indoor ozone level, and extent of surface coverage on model outputs, which include time-dependent residue consumption, overall (base and residue) reactivity, 
and byproduct formation. The model is useful for understanding how residues affect ozone consumption and byproduct formation, and what common indoor environmental factors influence those ozone-residue reactions.

In this chapter, the model was applied to the chamber experiments described in Chapter 3. With reasonable input parameter values, the model was able to recreate the time-profile of chamber ozone level for some materials better than others. It appears that some controlling factors of ozone consumption are not accounted for in the current model. In the future, the model could also be verified with surface residue chemistry experiments in the laboratory with base materials and residues that have better known properties.

One primary objective in modeling ozone-surface reactions is to explain the phenomenon of aging - the decrease in ozone reactivity of material during its exposure to ozone. This model captured the effect of a residue being consumed from top layer of a surface. This model could be combined with previous efforts to explain ozone-aging that incorporate diffusion of the ozone into the material (Morrison, 1999). One process that has not been modeled, which may play a role in ozone reactivity, is the diffusion of reactive compounds through the material. This line of research would conceivably be helpful in elucidating the role of aging and regeneration in surface material reactivity and byproduct formation.

Surface temperature variation is likely important for real indoor surface ozone uptake and byproduct emissions The effects of surface temperature were not investigated in this model, but temperature may affect volatilization of surface compounds and 
possibly the chemical reaction rate on the surface. This issue could be the focus of future studies of ozone-surface chemistry.

The scope of the surface reaction model presented in Chapter 4 was limited to primary ozone reactions, meaning that ozone reactions with byproducts were not considered, and radical production and subsequent reactions were not considered. An important next step in ozone-surface chemistry is to measure the production of hydroxyl and nitrate radicals from surface reactions in indoor settings. Radical chemistry has been well-studied in atmospheric chemistry, and those detection techniques could be employed in experiments that test indoor conditions. A more thorough understanding of the chemistry could be used to increase the complexity of this model and would provide better tools to predict byproduct formation in the indoor environment.

\subsection{Ozone-induced oxidation of surface-bound permethrin}

For gas-phase ozone reactions to be important for indoor air quality, the reaction rate typically must compete with the air-exchange rate. However, reactions on surfaces are not subject to the same time constraint and there is the opportunity for even slowly reacting compounds to be oxidized by ozone and ozone-induced radicals. In Chapter 5, I explored the potential for phosgene to be formed from ozone reactions with permethrin, a surface-bound insecticide used in commercial passenger airplanes. From a literature search, I determined that the likely ranges of cabin ozone levels and permethrin surface concentrations in "disinsected" cabins were potentially sufficient for phosgene formation at levels of concern based on phosgene health guidelines. 
An analysis method was developed that involved derivatizing phosgene in a liquid solution by bubbling phosgene-containing air through a cooled impinger. The derivative was analyzed by GC-MS operated in SIM mode, and the lower limit of detection was determined to be 2 ppb using the experimental conditions employed in this study. Experiments were performed using this derivatization technique to detect phosgene. In these experiments cabin materials and glass plates were coated in permethrin and exposed to ozone at cabin-relevant conditions ( $>150$ ppb supply $\mathrm{O}_{3}, 4.5 \mathrm{~h}^{-1}$ air exchange rate, $<1 \%$ relative humidity, and $\sim 1700 \mathrm{ng} \mathrm{cm}^{-2}$ ). Significant reaction of ozone by cabin materials, but not glass, was observed. Secondary formation of the hydroxyl radical $(\mathrm{OH})$ was expected to result from ozone-material reactions and $\mathrm{OH}$ was not scavenged. Phosgene formation was not detected in these experiments. Reaction of permethrin with ozone appears to be very slow because chlorine atoms greatly reduce the electron density of the neighboring double bond where ozone would react. Adjacent chlorine atoms have been shown to slow reactions with alkenes in the gas phase, but the effect on surface-bound compounds has not previously been reported.

A model of ozone transport and uptake was used to estimate the upper limit on ozone-permethrin reactivity to be $10^{-7}$. This model was also used to estimate that phosgene formation, if it occurs, is not likely to produce levels of phosgene that exceed a stringent phosgene health guideline that appears to be appropriate for cabin passengers and crew. I estimated that aircraft cabin phosgene level would likely be less than $1 \mu \mathrm{g}$

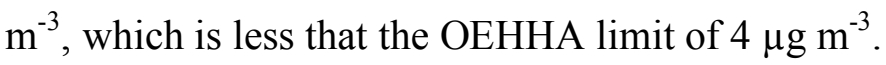

Based on these experiments, it was determined that the likely reaction ozonepermethrin byproduct, phosgene, is not likely to be formed at levels that would warrant 
concern in aircraft cabins. Ozone-initiated oxidation is not expected to be a significant path of degradation for permethrin or other chemically similar semivolatile organic compounds (SVOCs). However, with an even more sensitive detection technique and longer experiments, the formation of phosgene from permethrin under other conditions could be tested. This may be especially relevant for residential or public buildings (such as hospitals) that are likely to have a pesticide applied and to be important exposure environments for sensitive individuals. Although ozone-initiated reactions do not appear to be a major pathway of degradation for permethrin, reaction with ozone or $\mathrm{OH}$ is a possibility for other SVOCs. This degradation pathway and the resulting byproducts should be considered when determining the exposure pathway and ultimate toxicity of a chemical. This is important not only for chemicals applied directly to indoor spaces but also for SVOCs applied outdoors because there is evidence that they may accumulate indoors (McKone et al., 2007).

\subsection{Indoor ozone chemistry trends and solutions}

Evidence is mounting that exposure to ozone, and perhaps more importantly, exposure to its oxidation products are detrimental to comfort and health (Weschler, 2006). Ozone is a ubiquitous pollutant with wide-scale health impacts. For the first time in history, over half the world's population is living in urban (rather than rural) settings and trends indicate that populations will continue to become more urbanized. The current model of development and urbanization involves greater emissions of photochemical smog precursors that lead to the formation of ozone. In addition, models of global atmospheric chemistry indicate that ambient ozone may increase as climate change 
progresses (IPCC, 2007), although the trend in ozone generation is not certain and will vary by location. If regional and global ozone concentrations increase, exposure to ozone and the byproducts of ozone will also increase.

Ultimately, the most benefit would be reaped by lowering regional and global ozone levels. This solution, of course, requires time and wide-scale effort, but is achievable, as demonstrated by air pollution reductions in the Los Angeles Air Basin. In the near term, controlling ozone and ozone reactions in indoor environments seems to be the most practical way to control exposures.

One way to control ozone chemistry in indoor environments is to destroy ozone before it enters the space and is allowed to react with gas-phase constituents, surfaces, and humans. This can be a very effective strategy for systems where ventilation is tightly controlled, for instance in the aircraft cabin or in well-sealed buildings with mechanical ventilation systems. In this case it may be possible to use activated carbon or a metal catalyst to scrub ozone without producing oxidation byproducts. However, this strategy is not well suited for other environments where air enters in an uncontrolled way via infiltration or natural ventilation (i.e., through windows). Some novel studies have been performed at the University of Texas at Austin where unpainted gypsum board or sheets of activated carbon have been placed over large, exposed areas in residential settings to consume ozone without the formation of byproducts, thereby lowering the ozone concentration available to react with other surfaces. A benefit of this method of ozone removal is that it is passive and thus does not require energy for ozone removal. Energy, materials, and costs to produce and regenerate ozone catalysts could be considered in life 
cycle analysis. Other climate-friendly solutions that address exposure to ozone, its byproducts, and other indoor pollutants are a ripe topic of research. 


\section{References}

Alshawa, A., Russell, A.R., Nizkorodov, S.A., 2007. Kinetic analysis of competition between aerosol particle removal and generation by ionization air purifiers. Environmental Science \& Technology 41, 2498-2504.

Anderson, S.E., Wells, J.R., Fedorowicz, A., Butterworth, L.F., Meade, B.J., Munson, A.E., 2007. Evaluation of the contact and respiratory sensitization potential of volatile organic compounds generated by simulated indoor air chemistry. Toxicological Sciences 97, 355-363.

Appenzeller, C., Davies, H.C., 1992. Structure of stratospheric intrusions into the troposphere. Nature 358, 570-572.

Aschmann, S.M., Arey, J., Atkinson, R., 2002. OH radical formation from the gas-phase reactions of $\mathrm{O}_{3}$ with a series of terpenes. Atmospheric Environment 36, 43474355.

Asgharian, B., Price, O.T., 2007. Deposition of ultrafine (NANO) particles in the human lung. Inhalation Toxicology 19, 1045-1054.

Atkinson, R., 2000. Atmospheric chemistry of VOCs and NOx. Atmospheric Environment 34, 2063-2101.

Atkinson, R., Arey, J., 2003. Atmospheric degradation of volatile organic compounds. Chemical Reviews 103, 4605-4638.

Atkinson, R., Carter, W.P.L., 1984. Kinetics and mechanisms of the gas-phase reactions of ozone with organic compounds under atmospheric conditions. Chemical Reviews 84, 437-470. 
ATSDR (Agency for Toxic Substances \& Disease Registry), 2003. Toxicological profile for pyrethrins and pyrethroids. Atlanta, GA: U.S. Department of Health and Human Services, Public Health Service. $<$ http://www.atsdr.cdc.gov/tfacts155.html $>$.

ATSDR (Agency for Toxic Substances \& Disease Registry), 2007. Medical management guidelines for phosgene. Atlanta, GA: U.S. Department of Health and Human Services, Public Health Service. $<$ http://www.atsdr.cdc.gov/mhmi/mmg176.html>.

Avzianova, E.V., Ariya, P.A., 2002. Temperature-dependent kinetic study for ozonolysis of selected tropospheric alkenes. International Journal of Chemical Kinetics 34, $678-684$.

Bekarian, N., Payne-Sturges, D., Edmondson, S., Chism B., Woodruff, T.J., 2006. Use of point-of-sale data to track usage patterns of residential pesticides: methodology development. Environmental Health 5, 15.

Berger-Preiss, E., Koch, W., Behnke, W., Gerling, S., Kock, H., Elflein, L., Appel, K.E., 2004. In-flight spraying in aircrafts: Determination of the exposure scenario. International Journal of Hygiene and Environmental Health 207, 419-430.

Berger-Preiss, E., Koch, W., Gerling, S., Kock, H., Klasen, J., Hoffmann, G., Appel, K.E., 2006. Aircraft disinsection: Exposure assessment and evaluation of a new pre-embarkation method. International Journal of Hygiene and Environmental Health 209, 41-56. 
Bhangar, S., Cowlin, S.C., Singer, B.C., Sextro, R.G., Nazaroff, W.W., 2008. Ozone levels in passenger cabins of commercial aircraft on North American and transoceanic routes. Environmental Science \& Technology 42, 3938-3943.

Bischof, W., 1973. Ozone measurements in jet airliner cabin air. Water, Air, and Soil Pollution 2, 3-14.

Bornehag, C.G., Stridh, G., 2000. Volatile organic compounds (VOCs) in the Swedish housing stock. In: Seppänen, O., Säteri, J. (Eds.), Proceedings of the Healthy Buildings '00 Conference, Espoo. Vol.1, 437-442.

Brabets, R.I., Hersh, C.K., Klein, M.J., 1967. Ozone measurement survey in commercial jet aircraft. Journal of Aircraft 4, 59-64.

Brown, M.A., Petreas, M.X., Okamoto, H.S., Mischke, T.M., Stephens, R.D., 1993. Monitoring of malathion and its impurities and environmental transformation products on surfaces and in air following an aerial application. Environmental Science \& Technology 27, 388-397.

Calogirou, A., Larsen, B.R., Brussol, C., Duane, M., Kotzias, D., 1996. Decomposition of terpenes by ozone during sampling on Tenax. Analytical Chemistry 68, 14991506.

Cano-Ruiz, J.A., Kong, D., Balas, R.B., Nazaroff, W.W., 1993. Removal of reactive gases at indoor surfaces: Combining mass transport and surface kinetics. Atmospheric Environment 27A, 2039-2050.

CARB (California Air Resources Board), 2004. Indoor air quality guideline: Formaldehyde in the home. Sacramento, CA. $<$ http://www.arb.ca.gov/research/indoor/formaldGL08-04.pdf>. 
CDC (Centers for Disease Control and Prevention), 2006. Facts about phosgene. Atlanta, GA: U.S. Department of Health and Human Services. $<$ http://www.bt.cdc.gov/agent/phosgene/basics/facts.asp $>$.

CDPR (California Department of Pesticide Regulation), 2003. Environmental fate of permethrin. Sacramento, CA: California Environmental Protection Agency. $<$ www.cdpr.ca.gov/docs/emon/pubs/fatememo/permethrin.pdf $>$.

Chew, A.A., Atkinson, R., 1996. OH radical formation yields from the gas-phase reactions of $\mathrm{O}_{3}$ with alkenes and monoterpenes. Journal of Geophysical Research $101,28649-28653$.

Class, T.J., 1991. Determination of pyrethroids and their degradation products in indoor air and on surfaces by HRGC-ECD and HRGC-MS (NCI). Journal of High Resolution Chromatography 14, 446-450.

Cocker III, D.R., Clegg, S.L., Flagan, R.C., Seinfeld, J.H., 2001. The effect of water on gas-particle partitioning of secondary organic aerosol. Part I: $\alpha$-pinene/ozone system. Atmospheric Environment 35, 6049-6072.

Corsi, R.L., Siegel, J., Karamalegos, A., Simon, H., Morrison, G.C., 2007. Personal reactive clouds: Introducing the concept of near-head chemistry. Atmospheric Environment 41, 3161-3165.

Destaillats, H., Lunden, M.M., Singer, B.C., Coleman, B.K., Hodgson, A.T., Weschler, C.J., Nazaroff, W.W., 2006a. Indoor secondary pollutants from household product emissions in the presence of ozone: A bench-scale chamber study. Environmental Science \& Technology 40, 4421-4428. 
Destaillats, H., Singer, B.C., Lee, S.K., Gundel, L.A., 2006b. Effect of ozone on nicotine desorption from model surfaces: Evidence for heterogeneous chemistry. Environmental Science \& Technology 40, 1799-1805.

Dick, W.D., Ziemann, P.J., McMurry, P.H., 2007. Multiangle light-scattering measurements of refractive index of submicron atmospheric particles. Aerosol Science and Technology 41, 549-569.

DOT (U.S. Department of Transportation), 2007. Aircraft disinfection requirements. Washington, DC: U.S. Department of Transportation, Office of Transportation Policy, Safety and Health Team. $<$ http://ostpxweb.dot.gov/policy/SafetyEnergyEnv/disinsection.htm>.

Dowideit, P., von Sonntag, C., 1998. Reaction of ozone with ethene and its methyl- and chlorine-substituted derivatives in aqueous solution. Environmental Science \& Technology 32, 1112-1119.

EPA (U.S. Environmental Protection Agency), 1999. National-Scale Air Toxics Assessment. <http://www.epa.gov/ttn/atw/nata1999/index.html>.

EPA (U.S. Environmental Protection Agency), 2005. Toxicological review of phosgene. Washington, DC: U.S. Environmental Protection Agency, Integrated Risk Information System (IRIS). <http://www.epa.gov/iris/subst/0487.htm\#refinhal >.

EPA (U.S. Environmental Protection Agency), 2006. Reregistration eligibility decision (RED) for permethrin. Washington, DC: U.S. Environmental Protection Agency, Prevention, Pesticides, and Toxic Substances. $<$ http://www.epa.gov/oppsrrd1/REDs/factsheets/permethrin_fs.htm >. 
EPA (U.S. Environmental Protection Agency), 2008. Criteria air pollutants monitor values report for PM2.5 in United States in 2007. $<$ http://www.epa.gov/air/data/repsus.html?us $\sim$ USA $\sim$ United\%20States $>$.

Fan, Z., Lioy, P., Weschler, C., Fiedler, N., Kipen, H, Zhang, J., 2003. Ozone-initiated reactions with mixtures of volatile organic compounds under simulated indoor conditions. Environmental Science \& Technology 37, 1811-1821.

Feigenbrugel, V., Le Person, A., Le Calvé, S., Mellouki, A., Muños, A., Wirts, Z., 2006. Atmospheric fate of dichlorvos: photolysis and $\mathrm{OH}-$ initiated oxidation studies. Environmental Science \& Technology 40, 850-857.

Fick, J., Pommer, L., Andersson, B., Nilsson, C., 2001. Ozone removal in the sampling of parts per billion levels of terpenoid compounds: An evaluation of different scrubber materials. Environmental Science \& Technology 35, 1458-1462.

Finlayson-Pitts, B.J., Pitts, J.N., 2000. Chemistry of the Upper and Lower Atmosphere: Theory, Experiments and Application. Academic Press, New York.

Flemmer, M.M., Ham, J.E., Wells, J.R., 2007. Field and laboratory emission cell automation and control system for investigating surface chemistry reactions. Review of Scientific Instruments 78, Art. No. 014101.

Fruekilde, P., Hjorth, J., Jensen, N.R., Kotzias, D., Larsen, B., 1998. Ozonolysis at vegetation surfaces: A source of acetone, 4-oxopentanal, 6-methyl-5-hepten-2one, and geranyl acetone in the troposphere. Atmospheric Environment 32, 18931902.

Girman, J.R., Hadwen, G.E., Burton, L.E., Womble, S.E., McCarthy, J.F., 1999. Individual volatile organic compound prevalence and concentrations in 56 
buildings of the Building Assessment Survey and Evaluation (BASE) study.

Proceedings of the 8th International Conference on Indoor Air Quality and

Climate, Edinburgh. Vol. 2, 460-465.

Greene, R.S., Downing, D.T., Pochi, P.E., Strauss, J.S., 1970. Anatomical variation in the amount and composition of human skin surface lipid. The Journal of Investigative Dermatology 54, 240-247.

Grøntoft, T., Raychaudhuri, M.R., 2004. Compilation of tables of surface deposition velocities for $\mathrm{O}_{3}, \mathrm{NO}_{2}$ and $\mathrm{SO}_{2}$ to a range of indoor surfaces. Atmospheric Environment 38, 533-544.

Grosjean, E., Grosjean, D., 1997. Gas phase reaction of alkenes with ozone: Formation yields of primary carbonyls and biradicals. Environmental Science \& Technology $31,2421-2427$.

Haagen-Smit, A.J., 1952. Chemistry and physiology of Los Angeles smog. Industrial and Engineering Chemistry 44, 1342-1346.

Hakola, H., Arey, J., Aschmann, S.M., Atkinson, R., 1994. Product formation from the gas-phase reactions of $\mathrm{OH}$ radicals and $\mathrm{O}_{3}$ with a series of monoterpenes. Journal of Atmospheric Chemistry 18: 75-102.

Ham, J.E., Wells, J.R., 2008. Surface chemistry reaction of $\alpha$-terpineol [(R)-2-(4-methyl3-cyclohexenyl)isopropanol] with ozone and air on a glass and a vinyl tile. Indoor Air 18, 394-407.

Hand, J.L., Kreidenweis, S.M., 2002. A new method for retrieving particle refractive index and effective density from aerosol size distribution data. Aerosol Science and Technology 36, 1012-1026. 
Hearn, J.D., Smith, G.D., 2004. Kinetics and product studies for ozonolysis reactions of organic particles using aerosol CIMS. Journal of Physical Chemistry A 108, 10019-10029.

Holmstead, R.L., Casida, J.E., Ruzo, L.O., Fullmer, D.G.,1978. Pyrethroid photodecomposition: Permethrin. Journal of Agricultural and Food Chemistry 26, $590-595$.

Hubbard, H.F., Coleman, B.K., Sarwar, G., Corsi, R.L., 2005. Effects of an ozonegenerating air purifier on indoor secondary particles in three residential dwellings. Indoor Air 15, 432-444.

Hung, H.M., Ariya, P., 2007. Oxidation of oleic acid and oleic acid/sodium choride(aq) mixture droplets with ozone: Changes in hydroscopicityand role of secondary reactions. Journal of Physical Chemistry A 111, 620-632.

IPCC (Intergovernmental Panel on Climate Change), 2007. The Physical Science Basis. Contribution of Working Group I to the Fourth Assessment Report of the Intergovernmental Panel on Climate Change. Cambridge University Press, Cambridge, United Kingdom and New York, NY, USA, Chapter 10, p 793.

Jarvis, J., Seed, M.J., Elton, R.A., Sawyer, L., Agius, R.M., 2005. Relationship between chemical structure and the occupational asthma hazard of low molecular weight organic compounds. Occupational and Environmental Medicine 62, 243-250.

Kelly, T.J., Smith, D.L., Satola, J., 1999. Emission rates of formaldehyde from materials and consumer products found in California homes. Environmental Science \& Technology 33, 81-88. 
Khlystov, A., Stanier, C., Pandis, S.N., 2004. An algorithm for combining electrical mobility and aerodynamic size distributions data when measuring ambient aerosol. Aerosol Science and Technology 38 (Suppl. 1), 229-238.

Kim, J., Moin, P., Moser, R., 1987. Turbulence in fully developed channel flow at low Reynolds number. Journal of Fluid Mechanics 177, 133-166.

Kindler, T.P., Chameides, W.L., Wine, P.H., Cunnold, D.M., Alyea, F.N., 1995. The fate of atmospheric phosgene and the stratospheric chlorine loadings of its parent compounds: $\mathrm{CCl}_{4}, \mathrm{C}_{2} \mathrm{Cl}_{4}, \mathrm{C}_{2} \mathrm{HCl}_{3}, \mathrm{CH}_{3} \mathrm{CCl}_{3}, \mathrm{CHCl}_{3}$. Journal of Geophysical Research 100, 1235-1251.

Klepeis, N.E., Nelson, W.C., Ott, W.R., Robinson, J.P., Tsang, A.M., Switzer, P., Behar, J.V., Hern, S.C, Engelmann, W.H., 2001. The National Human Activity Pattern Survey (NHAPS): A resource for assessing exposure to environmental pollutants. Journal of Exposure Analysis and Environmental Epidemiology 11, 231-252.

Knudsen, H.N., Clausen, P.A., Wilkins, C.K., Wolkoff, P., 2007. Sensory and chemical evaluations of odorous emissions from building products with and without linseed oil. Building and Environment 42, 4059-4067.

Koch, S., Winterhalter, R., Uherek, E., Kolloff, A., Neeb, P., Moortgat, G.K., 2000. Formation of new particles in the gas-phase ozonolysis of monoterpenes. Atmospheric Environment 34, 4031-4042.

Lai, A.C.K., Nazaroff, W.W., 2000. Modeling indoor particle deposition from turbulent flow onto smooth surfaces. Journal of Aerosol Science 31, 463-476. 
Langer, S. Moldanová, J., Arrhenius, K., Ljungström, E., Ekberg, L., 2008. Ultrafine particles produced by ozone/limonene reactions in indoor air under low/closed ventilation conditions. Atmospheric Environment 42, 4149-4159.

Law, K.S., Plantevin, P.-H., Thouret, V., Marenco, A., Asman, W.A.H., Lawrence, M., Crutzen, P.J., Muller, J.-F., Hauglustaine, D.A., Kanakidou, M., 2000. Comparison between global chemistry transport model results and Measurement of Ozone and Water Vapor by Airbus In-Service Aircraft (MOZAIC) data. Journal of Geophysical Research 105, 1503-1525.

Lee, A., Goldstein, A.H., Keywood, M.D., Gao, S., Varutbangkul, V., Bahreini, R., Ng, N.L., Flagan, R.C., Seinfeld, J.H., 2006. Gas-phase products and secondary aerosol yields from the ozonolysis of ten different terpenes. Journal of Geophysical Research-Atmospheres 111, D07302.

Lee, K., Vallarino, J., Dumyahn, T., Özkaynak, H., Spengler, J.D., 1999. Ozone decay rates in residences. Journal of the Air \& Waste Management Association 49, $1238-1244$.

Leungsakul, S., Jaoui, M., Kamens, R.M., 2005. Kinetic mechanism for predicting secondary organic aerosol formation from the reaction of d-limonene with ozone. Environmental Science \& Technology 39, 9583-9594.

Liu, D.L., Nazaroff, W.W., 2001. Modeling pollutant penetration across building envelopes. Atmospheric Environment 35, 4451-4462.

Liu, Q.T., Chen, R., McCarry, B.E., Diamond, M.L., Bahavar, B., 2003. Characterization of polar organic compounds in the organic film on indoor and outdoor glass windows. Environmental Science \& Technology 37, 2340-2349. 
Liu, X., Mason, M., Krebs, K., Sparks, L., 2004. Full-scale chamber investigation and simulation of air freshener emissions in the presence of ozone. Environmental Science \& Technology 38, 2802-2812.

Ljubic, I., Sabljic, A., 2002. Theoretical study of the mechanism and kinetics of gasphase ozone additions to ethene, fluoroethene, and chloroethene: A multireference approach. Journal of Physical Chemistry A 106, 4745-4757.

Long, C.M., Suh, H.H., Koutrakis, P., 2000. Characterization of indoor particle sources using continuous mass and size monitors. Journal of the Air \& Waste Management Association 50, 1236-1250.

Maddalena, R.L., McKone, T.E., 2008. Insecticide exposures on commercial aircraft: A literature review and screening level assessment. Lawrence Berkley National Laboratory, Paper LBNL-1246E.

Matoba, Y., Takimoto, Y., Kato, T., 1998. Indoor behavior and risk assessment following residual spraying of d-phenothrin and d-tetramethrin. American Industrial Hygiene Association Journal 59, 191-199.

Matsunaga, S., Mochida, M., Kawamura, K., 2004. High abundance of gaseous and particulate 4-oxopentanal in the forestal atmosphere. Chemosphere 55, 11431147.

Matthews, T.G., Thompson, C.V., Wilson, D.L., Hawthorne, A.R., Mage, D.T., 1989. Air velocities inside domestic environments: An important parameter in the study of indoor air quality and climate. Environment International 15, 545-550. 
Mazumdar, S., Chen, Q., 2008. Influence of cabin conditions on placement and response of contaminant detection sensors in commercial aircraft. Journal of Environmental Monitoring 10, 71-81.

McKone, T.E., Castorina, R., Harnly, M.E., Kuwabara, Y., Eskenazi, B., Bradman, A., 2007. Merging models and biomonitoring data to characterize sources and pathways of human exposure to organophosphorous pesticides in the Salinas Valley of California. Environmental Science \& Technology 41, 3233-3240.

Meylan W.M., Howard P.H., 1993. Computer estimation of the atmospheric gas-phase reaction rate of organic compounds with hydroxyl radicals and ozone.

Chemosphere 26, 2293-2299.

Moise, T., Rudich, Y., 2002. Reactive uptake of ozone by aerosol-associated unsaturated fatty acids: Kinetics, mechanism, and products. Journal of Physical Chemistry A $106,6469-6476$.

Morrison, G.C., 1999. Ozone-surface interactions: Investigations of mechanisms, kinetics, mass transport, and implications of indoor air quality. Dissertation. University of California, Berkeley, Ca.

Morrison, G.C., Nazaroff, W.W., 2000. The rate of ozone uptake on carpets: Experimental studies. Environmental Science \& Technology 34, 4963-4968.

Morrison, G.C., Nazaroff, W.W., 2002a. Ozone interactions with carpet: Secondary emissions of aldehydes. Environmental Science \& Technology 36, 2185-2192.

Morrison, G.C., Nazaroff, W.W., 2002b. The rate of ozone uptake on carpet: Mathematical modeling. Atmospheric Environment 36, 1749-1756. 
Morrison, G.C., Nazaroff, W.W., Cano-Ruiz, J.A., Hodgson, A.T., Modera, M.P., 1998. Indoor air quality impacts of ventilation ducts: Ozone removal and emissions of volatile organic compounds. Journal of the Air \& Waste Management Association $48,941-952$.

Mueller, F.X., Loeb, L., Mapes, W.H., 1973. Decomposition rates of ozone in living areas. Environmental Science \& Technology 7, 342-346.

Muir, B., Cooper, D.B., Carrick, W.A., Timperley, C.M., Slater, B.J., Quick, S., 2005. Analysis of chemical warfare agents III. Use of bis-nucleophiles in the trace level determination of phosgene and perfluoroisobutylene. Journal of Chromatography A $1098,156-165$.

Müller, M.D., Kaifel, A.K., Weber, M., Tellman, S., Burrows, J.P., Loyola, D., 2003. Ozone profile retrieval from Global Ozone Monitoring Experiment (GOME) data using a neural network approach (Neural Network Ozone Retrieval System (NNORSY)). Journal of Geophysical Research 108, Art. No. 4497.

Nastrom, G.D., Holdemant, J.D., Perkins, P.J., 1980. Measurements of cabin and ambient ozone on B747 airplanes. Journal of Aircraft 17, 246-249.

Nazaroff, W.W., 2008. Inhalation intake fraction of pollutants from episodic indoor emissions. Building and Environment 43, 269-277.

Nazaroff, W.W., Cass, G.R., 1986. Mathematical modeling of chemically reactive pollutants in indoor air. Environmental Science \& Technology 20, 924-934.

Nazaroff, W.W., Cass, G.R., 1989. Mass-transport aspects of pollutant removal at indoor surfaces. Environment International 15, 567-584. 
Nazaroff, W.W., Weschler, C.J., 2004. Cleaning products and air fresheners: Exposure to primary and secondary air pollutants. Atmospheric Environment 38, 2841-2865.

Nazaroff, W.W., Weschler, C.J., Corsi, R.L., 2003. Indoor air chemistry and physics. Atmospheric Environment 37, 5451-5453.

Newchurch, M.J., Ayoub, M.A., Oltmans, S., Johnson, B., Schmidlin, F.J., 2003.Vertical distribution of ozone at four sites in the United States. Journal of Geophysical Research - Atmospheres 108, Art. No. 4031.

Ng, N.L., Kroll, J.H., Keywood, M.D., Bahreini, R., Varutbangkul, V., Flagan, R.C., Seinfeld, J.H., Lee, A., Goldstein, A.H., 2006. Contribution of first- versus second-generation products to secondary organic aerosols formed in the oxidation of biogenic hydrocarbons. Environmental Science \& Technology 40, 2283-2297.

Nicolaides, N., 1974. Skin lipids: Their biochemical uniqueness. Science 186, 19-26. NPTN (National Pesticide Telecommunications Network), 1998. Pyrethrins \& pyrethroids. Corvallis, OR: Oregon State University and U.S. Environmental Protection Agency, National Pesticide Information Center. $<$ http://npic.orst.edu/npicfact.htm>.

NRC, 2002. The Airliner Cabin Environment and the Health of Passengers and Crew. National Research Council Committee on Air Quality in Passenger Cabins of Commercial Aircraft. National Academy Press, Washington, D.C.

Oberdörster, G., 2001. Pulmonary effects of inhaled ultrafine particles. International Archives of Occupational and Environmental Health 74, 1-8. 
Odum, J.R., Hoffman, T., Bowman, F., Collins, D., Flagan, R.C., Seinfeld, J.H., 1996. Gas/particle partitioning and secondary organic aerosol yields. Environmental Science \& Technology 30, 2580-2585.

OEHHA (California Office Environmental Health Hazard Assessment), 1999. Determination of acute reference exposure levels for airborne toxicants: Acute toxicity summary: Phosgene. Sacramento, CA: California Environmental Protection Agency. <www.oehha.ca.gov/air/acute_rels/pdf/75445A.pdf>.

Okubayashi, S., Griesser, U.J., Bechtold, T., 2005. Moisture sorption/desorption behavior of various manmade cellulosic fibers. Journal of Applied Polymer Science 97, $1621-1625$.

OSHA (Occupational Safety \& Health Administration), 1986. Sampling and analytical methods: Phosgene. Washington, DC: US Department of Labor. $<$ http://www.osha.gov/dts/sltc/methods/organic/org061/org061.html $>$.

OSHA (Occupational Safety \& Health Administration), 2008a. Health guidelines: Formic acid. Washington, DC: US Department of Labor. $<$ http://www.osha.gov/SLTC/healthguidelines/formicacid/recognition.html $>$.

OSHA (Occupational Safety \& Health Administration), 2008b. Sampling and analytical methods: Acetic acid. Washington, DC: US Department of Labor. $<$ http://www.osha.gov/dts/sltc/methods/partial/pv2119/pv2119.html $>$.

Pandrangi, L.S., Morrison, G.C., 2008. Ozone interactions with human hair: Ozone uptake rates and product formation. Atmospheric Environment 42, 5079-5089.

Parmar, S.S., Grosjean, D., 1990. Laboratory tests of KI and alkaline annular denuders. Atmospheric Environment 24A, 2695-2698. 
Parrish, D.D., Millet, D.B., Goldstein, A.H., 2008. Increasing ozone concentration in marine boundary layer air inflow at the west coasts of North America and Europe. Atmospheric Chemistry and Physics Discussions 8, 13847-13901.

Peters, A., Wichmann, H.E., Tuch, T., Heinrich, J., Heyder, J., 1997. Respiratory effects are associated with the number of ultrafine particles. American Journal of Respiratory and Critical Care Medicine 155, 1376-1383.

Pollock, M.R., 1948. Unsaturated fatty acids in cotton wool plugs. Nature 161, 853.

Pope, C.A., Dockery, D.W., 2006. Health effects of fine particulate air pollution: Lines that connect. Journal of the Air \& Waste Management Association 56, 709-742.

Presto, A.A., Huff Hartz, K.E., Donahue, N.M., 2005. Secondary organic aerosol production from terpene ozonolysis. 2. Effect of NOx concentration. Environmental Science \& Technology 39, 7046-7054.

Reiss, R., Ryan, P.B., Koutrakis, P., Tibbetts, S.J., 1995. Ozone reactive chemistry on interior latex paint. Environmental Science \& Technology 29, 1906-1912.

Ruzo, L.O., Kimmel, E.C., Casida, J.E., 1986. Ozonides and epoxides from ozonization of pyrethroids. Journal of Agricultural and Food Chemistry 34, 937-940.

Sabersky, R.H., Sinema, D.A., Shair, F.H., 1973. Concentrations, decay rates, and removal of ozone and their relation to establishing clean indoor air. Environmental Science \& Technology 7, 347-353.

Salthammer, T., Schwarz, A., Fuhrmann, F., 1999. Emission of reactive compounds from wood-based furniture coatings. Atmospheric Environment 33, 75-84. 
Sarwar, G., Corsi, R., Allen, D., Weschler, C., 2003. The significance of secondary organic aerosol formation and growth in buildings: Experimental and computational evidence. Atmospheric Environment 37, 1365-1381.

Sarwar, G., Olson, D.A., Corsi, R.L., Weschler, C.J., 2004. Indoor fine particles: The role of terpene emissions from consumer products. Journal of the Air \& Waste Management Association 54, 367-377.

Segal-Rosenheimer, M., Dubowski, Y., 2007. Heterogeneous ozonolysis of cypermethrin using real-time monitoring FTIR techniques. Journal of Physical Chemistry C $111,11682-11691$.

Seinfeld, J.H., Pandis, S.N., 1998. Atmospheric chemistry and physics: from air pollution to climate change. Wiley, New York.

Shu, Y., Kwok, E.S.C., Tuazon, E.C., Atkinson, R., Arey, J., 1997. Products of the gasphase reactions of linalool with $\mathrm{OH}$ radicals, $\mathrm{NO}_{3}$ radicals, and $\mathrm{O}_{3}$. Environmental Science \& Technology 31, 896-904.

Singer, B.C., Coleman, B.K., Destaillats, H., Hodgson, A.T., Lunden, M.M., Weschler, C.J., Nazaroff, W.W., 2006a. Indoor secondary pollutants from cleaning product and air freshener use in the presence of ozone. Atmospheric Environment 40, 6696-6710.

Singer, B.C., Destaillats, H., Hodgson, A.T., Nazaroff, W.W., 2006b. Cleaning products and air fresheners: emissions and resulting concentrations of glycol ethers and terpenoids. Indoor Air 16, 179-191. 
Singer, B.C., Hodgson, A.T., Hotchi, T., Ming, K.Y., Sextro, R.G., Wood, E.E., Brown, N.J., 2007. Sorption of organic gases in residential rooms. Atmospheric Environment 41, 3251-3265.

Smith, A.M., Rigler, E., Kwok, E.S.C., Atkinson, R., 1996. Kinetics and products of the gas-phase reactions of 6-methyl-5-hepten-2-one and trans-cinnamaldehyde with $\mathrm{OH}$ and $\mathrm{NO}_{3}$ radicals and $\mathrm{O}_{3}$ at $296 \pm 2 \mathrm{~K}$. Environmental Science \& Technology $30,1781-1785$.

Smith, K.R., 1988. Air pollution: Assessing total exposure in the United States. Environment 30, 10-15 and 33-38.

Spengler, J.D., Ludwig, S., Weker, R.A., 2004. Ozone exposures during trans-continental and trans-Pacific flights. Indoor Air 14 (Supplement 7), 67-73.

Spicer, C.W., Murphy, M.J., Holdren, M.W., Myers, J.D., MacGregor, I.C., Holloman, C., James, R.R., Tucker, K., Zaborski, R., 2004. Relate air quality and other factors to comfort and health symptoms reported by passengers and crew on commercial transport aircraft (Part I). American Society of Heating, Refrigerating, and Air Conditioning Engineers (ASHRAE Project 1262-TRP), Atlanta, Georgia.

Stephens, S., Rossi, M.J., Golden, D.M., 1986. The heterogeneous reaction of ozone on carbonaceous surfaces. International Journal of Chemical Kinetics 18, 1133-1149.

Sutton, P.M., Vergara, X., Beckman, J., Nicas, M., Das, R., 2007. Pesticide illness among flight attendants due to aircraft disinsection. American Journal of Industrial Medicine 50: 345-356. 
Tamás, G., Weschler, C.J., Bakó-Biró, Z., Wyon, D.P., Strøm-Tejsen, P., 2006. Factors affecting ozone removal rates in a simulated aircraft cabin environment. Atmospheric Environment 40, 6122-6133.

Thornberry, T., Abbatt, J.P.D., 2004. Heterogeneous reaction of ozone with liquid unsaturated fatty acids: Detailed kinetics and gas-phase product studies. Physical Chemistry Chemical Physics 6, 84-93.

Turpin, B.J., Lim, H.J., 2001. Species contributions to $\mathrm{PM}_{2.5}$ mass concentrations: Revisiting common assumptions for estimating organic mass. Aerosol Science \& Technology 35, 602-610.

Uhde, E., Salthammer, T., 2007. Impact of reaction products from building materials and furnishings on indoor air quality-A review of recent advances in indoor chemistry. Atmospheric Environment 41, 3111-2128.

Utter, R.G., Burkholder, J.B., Howard, C.J., Ravishankara, A.R., 1992. Measurement of the mass accommodation coefficient of ozone on aqueous surfaces. Journal of Physical Chemistry 96, 4973-4979.

Vartiainen, E., Kulmala, M., Ruuskanen, T.M., Taipale, R., Rinne, J., Vehkamäki, H., 2006. Formation and growth of indoor air aerosol particles as a result of dlimonene oxidation. Atmospheric Environment 40, 7882-7892.

Wainman, T., Zhang, J.F., Weschler, C.J., Lioy, P.J., 2000. Ozone and limonene in indoor air: A source of submicron particle exposure. Environmental Health Perspectives 108, 1139-1145. 
Wang, H., Morrison, G.C., 2006. Ozone-initiated secondary emission rates of aldehydes from indoor surfaces in four homes. Environmental Science \& Technology 40, $5263-5268$.

Wells, J.R., Morrison, G.C., Coleman, B.K., 2008. Kinetics and reaction products of ozone and surface-bound squalene. Journal of ASTM International 5, JAI101629.

Weschler, C.J., 2000. Ozone in indoor environments: Concentration and chemistry. Indoor Air 10, 269-288.

Weschler, C.J., 2004. New directions: Ozone-initiated reaction products indoors may be more harmful than ozone itself. Atmospheric Environment 38, 5715-5716.

Weschler, C.J., 2006. Ozone's impact on public health: Contributions from indoor exposures to ozone and products of ozone-initiated chemistry. Environmental Health Perspectives 114, 1489-1496.

Weschler, C.J., Hodgson, A.T., Wooley, J.D., 1992. Indoor chemistry: Ozone, volatile organic compounds, and carpets. Environmental Science \& Technology 26, 23712377.

Weschler, C.J., Shields, H.C., 1997. Measurements of the hydroxyl radical in a manipulated but realistic indoor environment. Environmental Science \& Technology 31, 3719-3722.

Weschler, C.J., Shields, H.C., 1999. Indoor ozone/terpene reactions as a source of indoor particles. Atmospheric Environment 33, 2301-2312.

Weschler, C.J., Shields, H.C., 2003. Experiments probing the influence of air exchange rates on secondary organic aerosols derived from indoor chemistry. Atmospheric Environment 37, 5621-5631. 
Weschler, C.J., Wisthaler, A., Cowlin, S., Tamás, G., Strøm-Tejsen, P., Hodgson, A.T., Destaillats, H., Herrington, J., Zhang, J., Nazaroff W.W., 2007. Ozone-initiated chemistry in an occupied simulated aircraft cabin. Environmental Science \& Technology 41, 6177-6184.

Williams, B.J., Goldstein, A.H., Kreisberg, N.M., Hering, S.V., 2006. An in-situ instrument for speciated organic composition of atmospheric aerosols: Thermal Desorption Aerosol GC/MS-FID (TAG). Aerosol Science and Technology 40, 627-638.

Wisthaler, A., Tamás, G., Wyon, D.P., Strøm-Tejsen, P., Space, D., Beauchamp, J., Hansel, A., Märk, T.D., and Weschler, C.J., 2005. Products of ozone-initiated chemistry in a simulated aircraft environment. Environmental Science \& Technology 39, 4823-4832.

Wolkoff, P., Clausen, P.A., Nielsen, P.A., 1995. Application of the Field and Laboratory Emission Cell "FLEC" - performance study, intercomparison study, and case study of damaged linoleum in an office, Indoor Air 5, 196-203.

Wolkoff, P., Wilkins, C.K., Clausen, P.A., Nielsen, G.D., 2006. Organic compounds in office environments - sensory irritation, odor, measurements and the role of reactive chemistry. Indoor Air 16, 7-19.

World Health Organization (WHO), 1995. Report of the information consultation on aircraft disinsection. International Programme on Chemical Safety: Geneva, Switzerland, World Health Organization. 
Yeh, H.C., Cuddihy, R.G., Phalen, R.F., Chang, I.Y., 1996. Comparisons of calculated respiratory tract deposition of particles based on the proposed NCRP model and the new ICRP66 model. Aerosol Science and Technology 25, 134-140.

Zahardis, J., Petrucci, G.A., 2007. The oleic acid-ozone heterogeneous reaction system: Products, kinetics, secondary chemistry, and atmospheric implications of a model system - a review. Atmospheric Chemistry and Physics 7, 1237-1274.

Zhang, J., Huff Hartz, K.E., Pandis, S.N., Donahue, N.M., 2006. Secondary organic aerosol formation from limonene ozonolysis: Homogeneous and heterogeneous influences as a function of NOx. Journal of Physical Chemistry A 110, 1105311063.

Zhang, L.Z., Niu, J.L., 2003. Laminar fluid flow and mass transfer in a standard field and laboratory emission cell. International Journal of Heat and Mass Transfer 46, 91100.

Zhang, T., Chen, Q.Y., 2007. Novel air distribution systems for commercial aircraft cabins. Building and Environment 42, 1675-1684.

Zuraimi, M.S., Weschler, C.J., Tham, K.W., Fadeyi, M.O., 2007. The impact of building recirculation rates on secondary organic aerosols generated by indoor chemistry. Atmospheric Environment 41, 5213-5223. 